$$
D O E / W S P O--98004012
$$

Enclosure

\title{
QUARTERLY ENVIRONMENTAL DATA SUMMARY FOR FOURTH QUARTER 1997
}

In support of the Weldon Spring Site Remedial Action Project Federal Facilities Agreement, a copy of the Quarterly

Environmental Data Summary (QEDS) for the fourth quarter of 1997 is enclosed.

The data presented constitute the QEDS. The data were received from the contract laboratories, verified by the Weldon spring Site verification group and, except for air monitoring data and site KPA generated data (uranium analyses), merged into the data base during the fourth quarter of 1997. Air monitoring data presented are the most recent complete sets of quarterly data. Air data are not stored in the data base and KPA data are not merged into the regular data base.

Significant data, defined as data values that have exceeded defined "above normal" level 2 values, are discussed in this letter for Environmental Monitoring Plan (EMP) generated data only. Above normal level 2 values are based, in ES\&H procedures, on historical high values, DOE Derived Concentration Guides (DCGs), NPDES limits and other guidelines. The procedures also establish actions to be taken in response to such data.

All data received and verified during the fourth quarter were within a permissible range of variability except for those detailed below. Select data from groundwater and air media are described in this QEDS for Fourth Quarter 1997.

The attached tables present the most recent data. for air and the data merged into the data base during the fourth quarter 1997 for groundwater, NPDES, surface water, and springs.

Graphs showing concentrations of selected contaminants of concern at some of the critical locations have also been included in this QEDS. The graphs are discussed in the separate sections.

\section{NPDES}

The uranium concentrations at the three major NPDES outfalls are graphed for January 1997 to December 1997. The graphs are located at the beginning of the NPDES tables. For comparison purposes, the annual average for 1996 is also graphed. Also, the derived concentration guide (DCG) of $600 \mathrm{pCi} / 1$ for uranium is noted. The DCG is the concentration of a radionuclide in air or water that, under conditions of continuous exposure for one year by one exposure mode (i.e., ingestion), would result in an effective dose equivalent of $100 \mathrm{mrem}$. A graph of historical annual averages for the three outfalls is also attached.

Outfall NP-0010, the CMSA sedimentation basin outfall, has remained below the 1996 annual average (50.1 pCi/l) in the fourth quarter for uranium and, because the area has been remediated, it is expected to remain so. Because NP-0010 is a minor outfall, it is not graphed. 


\section{DISCLAIMER}

Portions of this document may be illegible electronic image products. Images are produced from the best available original document. 
Outfall NP-0002 remained well below the 1996 annual average for uranium (54 pCi/l) for each of the three months of the quarter. The final 1997 annual average is $14 \mathrm{pCi} / 1$, well below the 1996 annual average of $54 \mathrm{pCi} / 1$. The reduction is due to the remediation of a majority of the NP-0002 watershed.

Outfall NP-0005 also remained well below the 1996 annual average for uranium (101 pCi/l) for each of the three months of the quarter. The final 1997 annual average is $19 \mathrm{pCi} / 1$, greatly reduced from the 1996 average. The reduction is due to remediation of a large portion of the NP-0005 watershed.

The uranium levels at NP-0003 were lower than the 1996 annual average (88 pCi/l) for October and November and slightly higher for December. The final 1997 annual average is $143 \mathrm{pCi} / 1$, an increase over the 1996 annual average. While most of the outfall NP-0003 watershed has been remediated, it contains Ash Pond, where contaminated soils, concrete, etc., are being stored. This has probably contributed to the higher levels at NP-0003. Water is allowed to continuously discharge from the Ash Pond area only if its uranium activity is measured to be less than $600 \mathrm{pCi} / 1$. The volume of runoff from Ash Pond is low in comparison to the remainder of the watershed. Stabilization of soil piles stored in the Ash Pond area will likely help reduce uranium levels in the storm water run off. However, when material is removed for placement in the cell, fluctuating uranium levels are expected.

All NPDES data merged within the fourth quarter were within normal ranges. Data values that were not resolved in previous QEDS are discussed below.

\section{NRDES STORM WATER OUTFALIS}

- Sample NP-0002-081597

This sample for settleable solids was reported in the third quarter 1997 QEDS. Settleable solids were $55 \mathrm{ml} / \mathrm{l} / \mathrm{hr}$. Offsite contributions to the outfall were suspected of causing solids to precipitate from site water faster than they otherwise would. Samples collected subsequently were all within permitted limits at $<0.1 \mathrm{ml} / \mathrm{l} / \mathrm{hr}$. Upstream monitoring for settleable solids will continue to help discriminate site-related settleable solids levels from those caused by upstream influences.

The analytical results for the NPDES outfalls are reported in the quarterly discharge monitoring report as well as in this report.

\section{GROUNDWATER}

\section{Chemical Plant}

\section{Site Water Treatment Plant and Temporary Storage Area}

- Samples GW-2035-Q297 and GW-2035-Q397

The second quarter uranium concentration, at $3.59 \mathrm{pCi} / 1$, was above baseline (1.93 pCi/l) for this location. On-site KPA 
analysis of a sample of this location during third quarter 1997 indicated that the value was within normal range at $0.51 \mathrm{pCi} / 1$. In addition, the third quarter uranium result for this location from the off-site laboratory was reported as $0.391 \mathrm{pCi} / 1$, which is also within the normal range.

- Samples GW-2036-Q297 and GW-2036-Q397

The uranium concentration, at $6.59 \mathrm{pCi} / 1$, for this location west of the TSA was reported above baseline (1.64 pCi/1) for the second quarter of 1997 . On-site KPA results from a resampling of this location indicated that the uranium level was slightly elevated at $1.8 \mathrm{pCi} / 1$; however, the third quarter off-site analytical result was within normal range at $0.694 \mathrm{pCi} / 1$.

- Samples GW-2038-Q397 and GW-2039-Q397

Metals reported above baseline at these two locations are suspected to be in error. The third quarter value for lead at location MW-2038 was reported as $17.7 \mathrm{ug} / 1$, above the baseline value of $1.65 \mathrm{ug} / 1$. The arsenic value at location $M W-2039$ (baseline $2.43 \mathrm{ug} / 1$ ) was reported as $107 \mathrm{ug} / 1$. Both locations were resampled and the values for these metals were reported as non-detections (below $2.0 \mathrm{ug} / \mathrm{l}$ for lead and below $2.1 \mathrm{ug} / \mathrm{l}$ for arsenic).

\section{VOC Monitoring}

- Sample GW-2037-0997, GW-2037-1097, GW-2038-0997, GW-2038-1097, GW-3025-0997, GW-3025-1097, MW-S021-0997 and MW-S021-1097

Monthly monitoring for the Volatile Organic Compounds (VoCs) south and west of Raffinate Pits 3 and 4 at WSSRAP locations MW2037, MW-2038, MW-3025 and Department of Army location MW-S021 continued during third quarter 1997. Trichloroethene (TCE) concentrations at location $\mathrm{MW}-2037$ ranged from $1,100 \mathrm{ug} / 1$ to 1,280 ug/1. Slight decreases in TCE were reported for location MW-S021 where late third quarter 1997 values ranged from $540 \mathrm{ug} / \mathrm{l}$ to $592 \mathrm{ug} / 1$ as compared to early third quarter 1997 concentrations of up to $620 \mathrm{ug} / \mathrm{I}$. In addition, TCE slightly decreased at location MW-2038 where fourth quarter 1997 values ranged from $370 \mathrm{ug} / 1$ to $530 \mathrm{ug} / 1$ as compared to third quarter, 1997 values which ranged from $560 \mathrm{ug} / 1$ to $620 \mathrm{ug} / 1$. The TCE concentrations at these locations are plotted versus time on the attached figure.

Monthly sampling continued through fourth quarter 1997, and the results will be reported in the first quarter 1998 QEDS. The sampling schedule for 1998 includes bimonthly sampling of these locations.

\section{Enhanced Raffinate Pit Area Monitoring}

Enhanced raffinate pit area groundwater monitoring includes 
increased sampling frequency and additional analytical parameters over those listed in the 1997 Environmental Monitoring Program

(EMP) for selected locations in the southern and western portions of the site. The purpose of the changes is to provide increased capability of detecting impacts on groundwater from disturbance of raffinate pit sludge and soil during ongoing remediation efforts. The monitoring includes monthly sampling for 11 groundwater monitoring locations (MW-2037, MW-2038, MW-2039, MW3003, MW-3023, MW-3024, MW-3025, MW-3027, MW-4001, MW-4002, and MW-4006). The analytical parameters for the 11 locations include nitrate, sulfate, metals (Toxicity List), and total uranium (onsite KPA).

No impacts on groundwater attributable to recent raffinate pit activities were detected during the fourth quarter 1997 monitoring period.

\section{Disposal Cell Detection Monitoring}

- Sample GW-2048-Q397-A

Groundwater sampling for the fourth quarter 1997 Disposal Cell Detection Monitoring system (MW-2032, MW-2045, MW-2046, MW-2047, and $\mathrm{MW}-2048$ ) was completed in October 1997. The analytical results from this event were within the expected ranges (based upon three previous sampling events) for all parameters.

The sampling results from this first year of detection monitoring were statistically evaluated quarterly, and these evaluations will be used to determine baseline groundwater quality conditions against which subsequent monitoring results (during and after waste placement) will be compared.

\section{Weldon Spring Quarry}

\section{Quarry Water Treatment Plant}

- Samples GW-1035-Q397, GW-1036-Q397, GW-1037-Q397, GW-1040-Q397

Chloride levels for groundwater monitoring locations in the vicinity of the Quarry Water Treatment Plant remained above baseline through the third quarter of 1997. In addition to chloride, fluoride levels suddenly increased at these locations during the second quarter of 1997. St. Charles County began fluoridating their public water supply during June 1997 . It was suspected that the recent fluoride and chloride increases, and previous chloride (and related elevated metals) increases were related to a subsurface release of county water from water mains along Missouri State Highway 94. Locations MW-1035 (upgradient of the quarry water treatment plant adjacent to Highway 94) and MW-1036 show the most elevated levels of these ions. The PMC coordinated a field inspection and water line pressure test with St. Charles County Public water Supply District Number 2 to investigate this possibility. No leaks along the water Iines were detected. Surface water samples were collected from along the Little Femme Osage Creek to determine if creek water is contributing to the abnormal groundwater quality, but no 
anomalous levels were detected. The monitoring wells will continue to be sampled, and analytical results and any findings from the investigation will be reported in subsequent QEDS. These locations are being evaluated to statistically determine whether baseline adjustments are required.

\section{Quarry Vicinity}

No above-normal contaminant values were reported during the fourth quarter reporting period. Groundwater quality in the quarry vicinity continues to show improvement at locations along the quarry rim for total uranium and nitroaromatic compounds.

\section{St. Charles County Well Field}

No elevated levels of contaminants originating from the Weldon. Spring Quarry were reported during the fourth quarter 1997 data review. Uranium activity results from the last four sampling events at the St. Charles County Well Field are shown on the attached figure.

\section{SPRINGS}

No elevated contaminants were reported in samples from springs during the fourth quarter of 1997 . Total uranium values at Burgermeister spring are plotted versus time on the attached figure.

\section{SURFACE WATER}

No elevated surface water contaminants were detected in samples collected during the fourth quarter of 1997.

\section{AIR}

Data for air monitoring locations are attached. Graphs of the monitoring results are located in front of the tables and discussed below.

The gross alpha concentrations for air particulate monitoring for fourth quarter 1997 are graphed along with the background level (which is based on the first quarter 1996 to the fourth quarter 1997 average concentration), and the background plus 3 standard deviations shown for comparison. The background location is AP4012, at the Daniel Boone Elementary School in New Melle.

The gamma exposure from environmental TLD monitoring results for the third quarter 1997 are graphed with the background and the background plus 25 mrem quarterly committed effected dose equivalent (TEDE) shown for comparison. The background level is based on monitoring conducted from fourth quarter 1996 to third quarter 1997. The 25 mrem Total Effective Dose Equivalent (TEDE) is based on one-fourth of the annual public dose limit of 100 mrem (TEDE) established in DOE order, 5400.5. The background locations are TD-4005 (west of the army site) and TD-4009 (Daniel Boone Elementary School, New Melle). 
The alpha track radon and thoron monitoring results are graphed for the third quarter of 1997. The background level (based on fourth quarter 1996 to third quarter 1997 monitoring) and the Derived Concentration Guide (DCG) for radon and thoron are shown for comparison. The DCG is a reference value for protection of the public and the environment contained in DOE order 5400.5 . Although the concentrations for some locations are above the DCG, the locations are all within the site boundary. The background locations are RD-4005 (west of the army site) and RD-4009 (Daniel Boone Elementary School, New Melle).

Two above normal results for air monitoring occurred during the third quarter and they are discussed below.

\section{RAFFINATE PITS}

RD-3001-0397, RD-3003-0397

During the third quarter of 1997, the thoron concentrations obtained at the alpha-track monitoring stations RD-3001 (northwest of raffinate pit 4) and RD-3003 (south of raffinate pit 4 and west of the TSA) indicated $1.1 \mathrm{pCi} / 1$ and $1.2 \mathrm{pCi} / 1$, , respectively. These levels were $1 \mathrm{pCi} / 1$ or more above background and were thus considered "above normal" levels for a site perimeter monitoring location. These elevated concentrations were caused by increased exposed surface area of thoron generating waste (i.e., sludge) and resulted in a higher thoron release to the surrounding atmosphere. Dose assessment was performed based on these measurements, and the assessment indicated an Effective Dose Equivalent (EDE) of less than 0.1 mrem to members of the public in the area. A follow-up sample was collected during the fourth quarter of 1997 . The results have not yet been received, but they will be reported in the first quarter 1998 QEDS. Sludge consolidation is complete and, therefore, thoron concentrations are expected to decrease.

\section{SUMMARY}

The data described in this report are highlighted as being above prescribed baseline values, varying from historical ranges, or being above regulatory guidelines or limits. As a result, they are subject to more focused attention by the WSSRAP Environmental Protection Group. Continuous trends are monitored to determine the possible need for additional action. Except for the highlighted data, all other indicators subject to reporting in the QEDS were within historic range or below reporting criteria.

If you have any questions, please contact the WSSRAP Community Relations Department at(314)441-8086. 


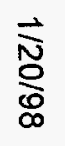

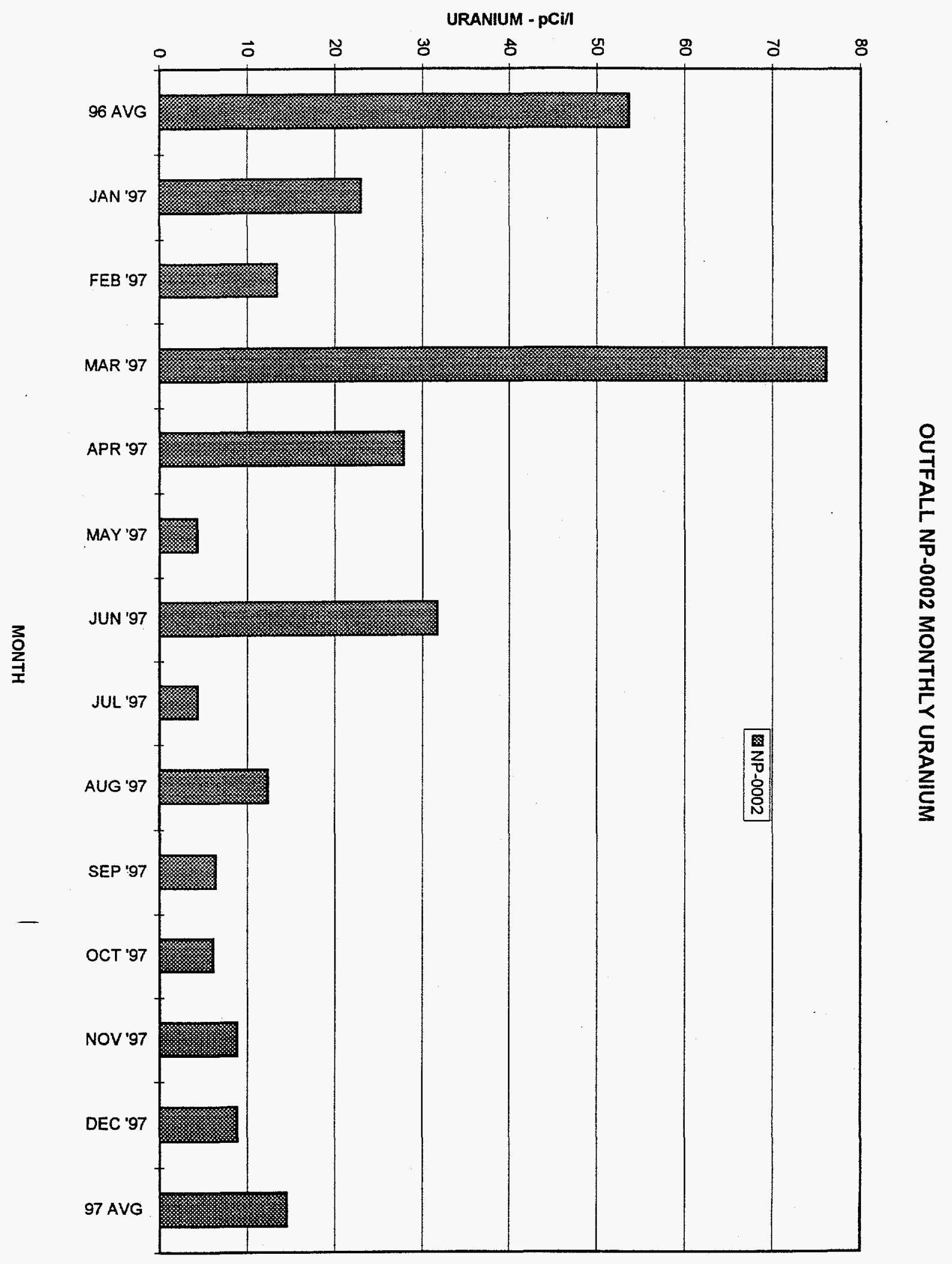




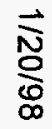

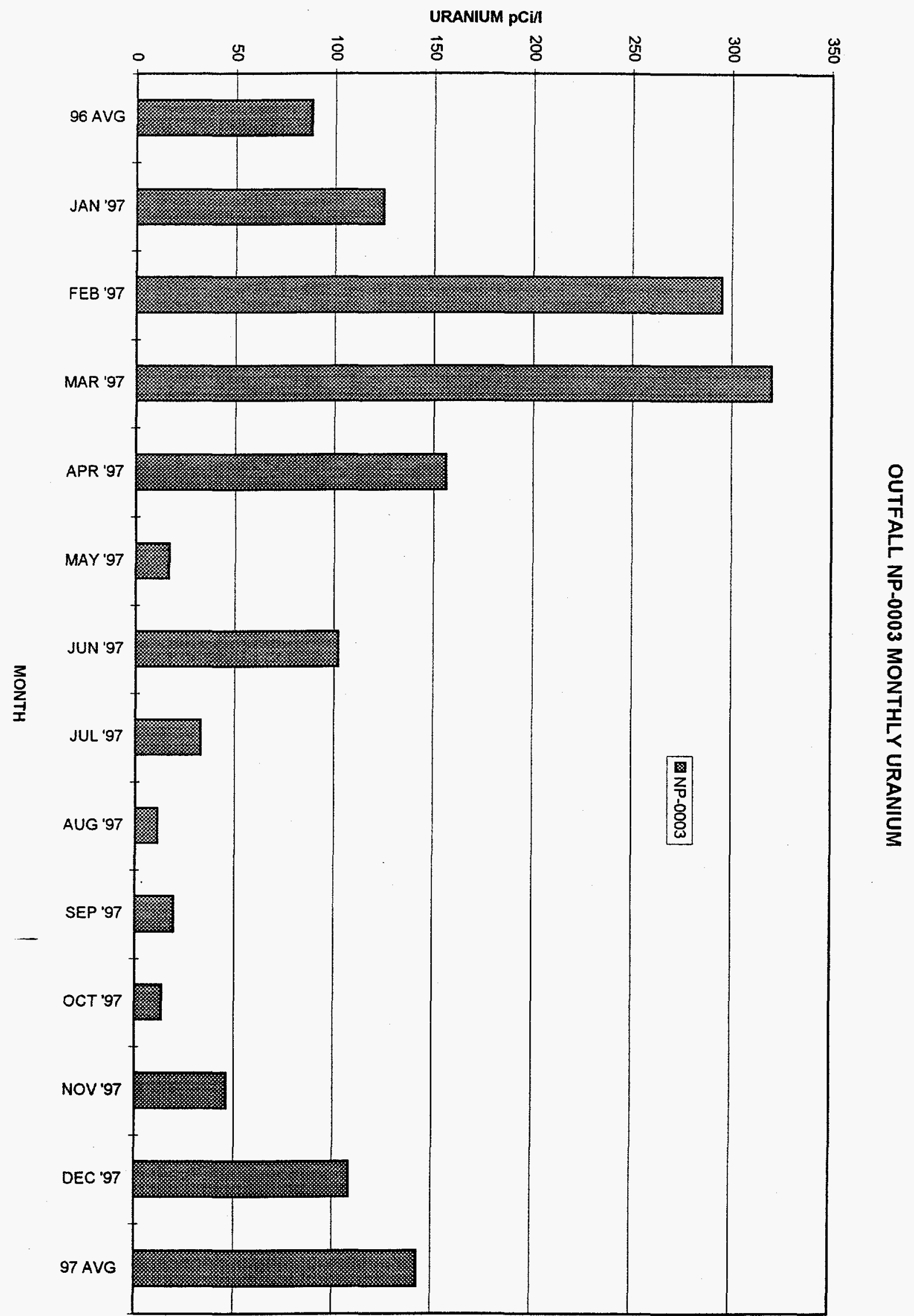


$\underset{\substack{0 \\ 0}}{0}$

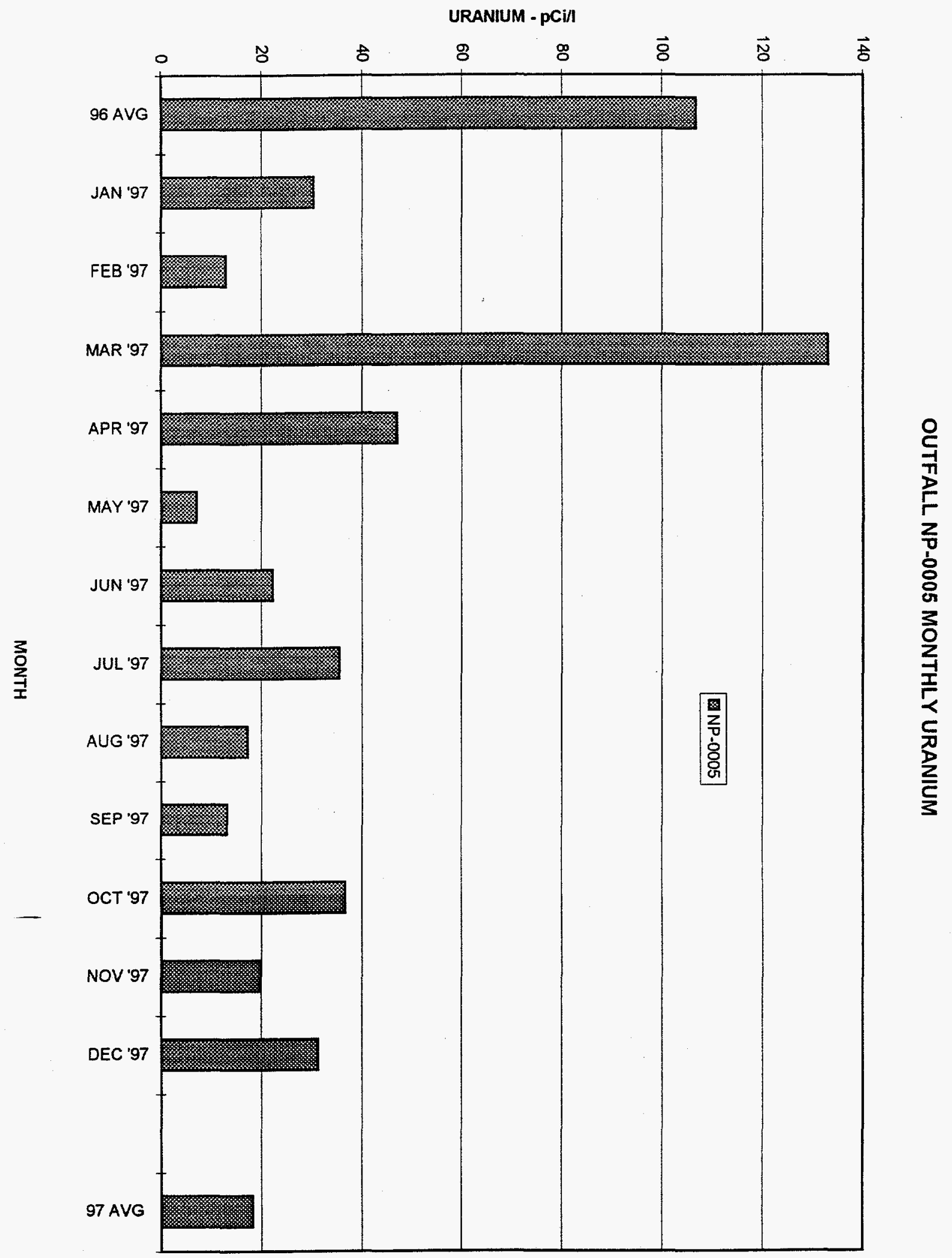




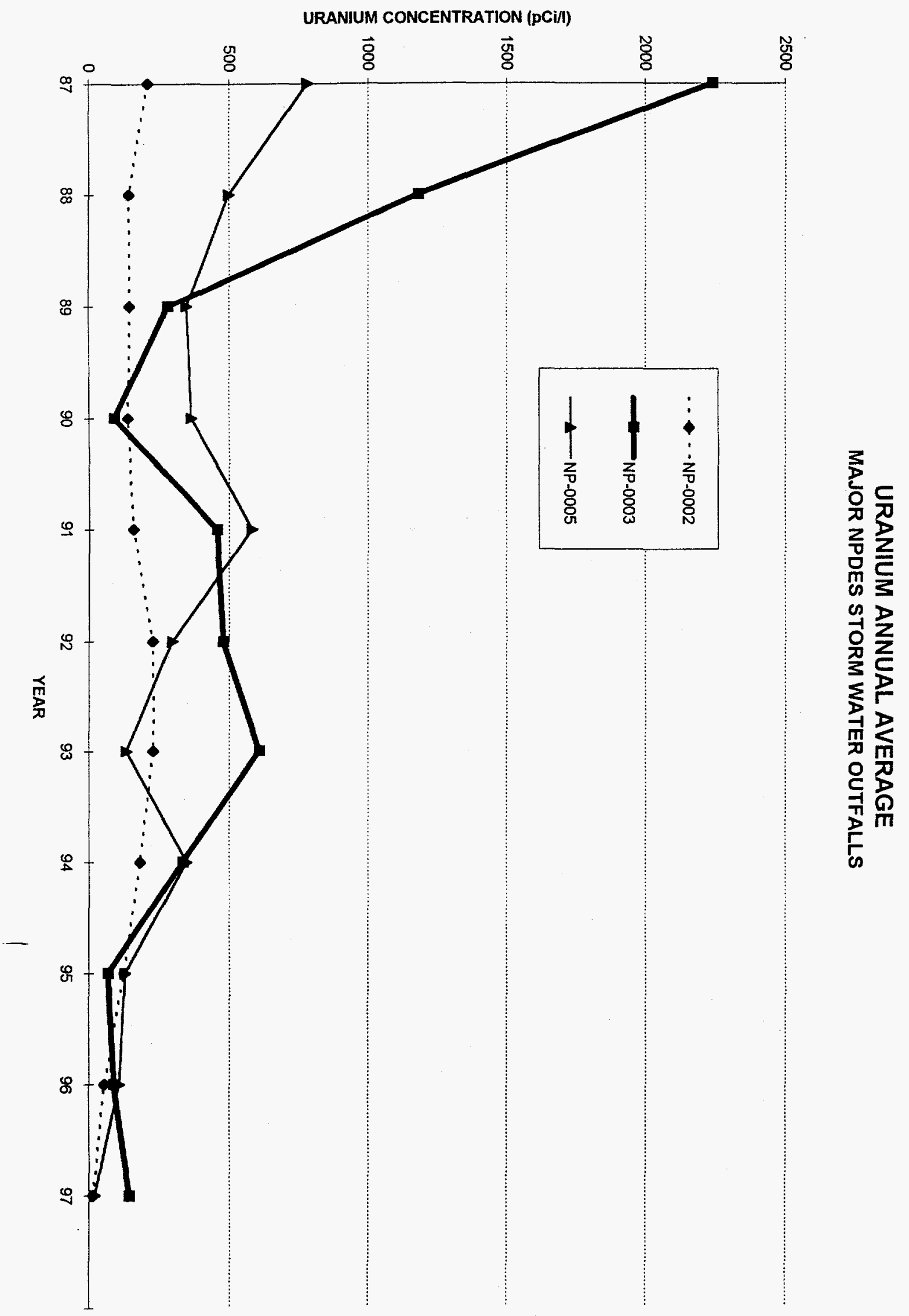


NPDES

DATA MERGED DURING FOURTH QUARTER 1997

\begin{tabular}{|c|c|c|c|c|c|c|}
\hline WSSRAP_ID & DATE_SAM & PARAMETER & CONC & $\mathrm{DL}$ & UNITS & METHOD \\
\hline 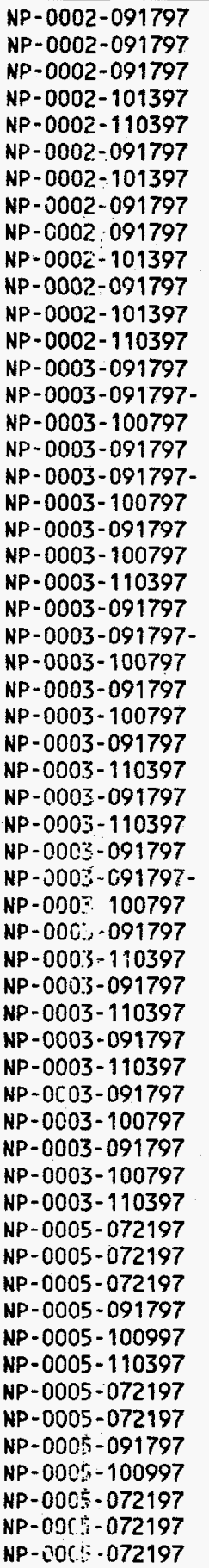 & $\begin{array}{l}09 / 17 / 97 \\
09 / 17 / 97 \\
09 / 17 / 97 \\
10 / 13 / 97 \\
11 / 03 / 97 \\
09 / 17 / 97 \\
10 / 13 / 97 \\
09 / 17 / 97 \\
09 / 17 / 97 \\
10 / 13 / 97 \\
09 / 17 / 97 \\
10 / 13 / 97 \\
11 / 03 / 97 \\
09 / 17 / 97 \\
09 / 17 / 97 \\
10 / 07 / 97 \\
09 / 17 / 97 \\
09 / 17 / 97 \\
10 / 07 / 97 \\
09 / 17 / 97 \\
10 / 07 / 97 \\
11 / 03 / 97 \\
09 / 17 / 97 \\
09 / 17 / 97 \\
10 / 07 / 97 \\
09 / 17 / 97 \\
10 / 07 / 97 \\
09 / 17 / 97 \\
11 / 03 / 97 \\
09 / 17 / 97 \\
11 / 03 / 97 \\
09 / 17 / 97 \\
09 / 17 / 97 \\
10 / 07 / 97 \\
09 / 17 / 97 \\
111 / 03 / 97 \\
09 / 17 / 97 \\
11 / 03 / 97 \\
09 / 17 / 97 \\
111 / 03 / 97 \\
09 / 17 / 97 \\
10 / 07 / 97 \\
09 / 17 / 97 \\
10 / 07 / 97 \\
11 / 03 / 97 \\
07 / 21 / 97 \\
07 / 21 / 97 \\
07 / 21 / 97 \\
09 / 17 / 97 \\
10 / 09 / 97 \\
11 / 03 / 97 \\
07 / 21 / 97 \\
07 / 21 / 97 \\
09 / 17 / 97 \\
10 / 09 / 97 \\
07 / 21 / 97 \\
07 / 21 / 97 \\
07 / 21 / 97\end{array}$ & $\begin{array}{l}\text { CHLORIDE } \\
\text { FLUORIDE } \\
\text { GROSS ALPHA } \\
\text { GROSS ALPHA } \\
\text { GROSS ALPHA } \\
\text { NITRATE-N } \\
\text { NITRATE-N } \\
\text { SULFATE } \\
\text { TOTAL SUSPENDED SOLIDS } \\
\text { TOTAL SUSPENDED SOLIDS } \\
\text { URANIUM, TOTAL } \\
\text { URANIUM, TOTAL } \\
\text { URANIUM, TOTAL } \\
\text { ARSENIC } \\
\text { ARSENIC } \\
\text { ARSENIC } \\
\text { CHROMIUM } \\
\text { CHROMIUM } \\
\text { CHROMIUM } \\
\text { GROSS ALPHA } \\
\text { GROSS ALPHA } \\
\text { GROSS ALPHA } \\
\text { LEAD } \\
\text { LEAD } \\
\text { LEAD } \\
\text { NITRATE-N } \\
\text { NITRATE-N } \\
\text { RADIUM-226 } \\
\text { RADIUM-226 } \\
\text { RADIUM-228 } \\
\text { RADIUM-228 } \\
\text { THALLIUM } \\
\text { THALLIUM } \\
\text { THALLIUM } \\
\text { THORIUM-228 } \\
\text { THORIUM-228 } \\
\text { THORIUM-230 } \\
\text { THORIUM-230 } \\
\text { THORIUM-232 } \\
\text { THORIUM-232 } \\
\text { TOTAL SUSPENDED SOLIDS } \\
\text { TOTAL SUSPENDED SOLIDS } \\
\text { URANIUM, TOTAL } \\
\text { URANIUM, TOTAL } \\
\text { URANIUM, TOTAL } \\
\text { ARSENIC } \\
\text { CHROMIUM } \\
\text { GROSS ALPHA } \\
\text { GROSS ALPHA } \\
\text { GROSS ALPHA } \\
\text { GROSS ALPHA } \\
\text { LEAD } \\
\text { NITRATE-N } \\
\text { NITRATE-N } \\
\text { NITRATE-N } \\
\text { RADIUM-226 } \\
\text { RADIUM-228 } \\
\text { THALLIUM }\end{array}$ & $\begin{array}{l}230 \\
0.390 \\
24.4 \\
69.91 \\
15.6 \\
0.810 \\
0.278 \\
43.8 \\
1220 \\
38.0 \\
6.28 \\
6.09 \\
8.83 \\
283 \\
4.5 \\
\text { ND } \\
10.4 \\
21.2 \\
\text { ND } \\
29.9 \\
26.9 \\
45.7 \\
9.7 \\
\text { ND } \\
\text { ND } \\
1.02 \\
0.025 \\
1.13 \\
0.306 \\
2.43 \\
1.48 \\
\text { ND } \\
\text { ND } \\
\text { ND } \\
\text { ND } \\
C 0.09 \\
60.05 \\
0.555 \\
\text { ND } \\
60.13 \\
280 \\
51.0 \\
19.2 \\
13.7 \\
45.8 \\
\text { ND } \\
\text { ND } \\
25.5 \\
13.0 \\
32.6 \\
17.9 \\
\text { ND } \\
\text { ND } \\
0.740 \\
\text { ND } \\
0.190 \\
1.37 \\
\text { ND }\end{array}$ & $\begin{array}{l}\text { 6.40 } \\
0.0200 \\
1.91 \\
13.9 \\
4.41 \\
0.00700 \\
0.0100 \\
0.900 \\
33.4 \\
2.00 \\
0.0643 \\
0.0752 \\
0.0203 \\
2.5 \\
4 \\
100 \\
0.5 \\
2 \\
10.0 \\
2.47 \\
4.15 \\
1.74 \\
1.2 \\
9.5 \\
50.0 \\
0.00700 \\
0.0100 \\
0.388 \\
0.200 \\
0.746 \\
1.32 \\
2.4 \\
7 \\
100 \\
0.172 \\
0.360 \\
0.0899 \\
0.279 \\
0.0649 \\
0.302 \\
16.7 \\
2.00 \\
0.0643 \\
0.0752 \\
0.0203 \\
100 \\
10.0 \\
4.49 \\
4.35 \\
4.19 \\
2.37 \\
50.0 \\
0.0100 \\
0.00700 \\
0.0100 \\
0.116 \\
1.32 \\
100 \\
\\
\\
\\
\\
\end{array}$ & $\begin{array}{l}M G / L \\
M G / L \\
P C I / L \\
P C I / L \\
P C I / L \\
M G / L \\
M G / L \\
M G / L \\
M G / L \\
M G / L \\
P C I / L \\
P C I / L \\
P C I / L \\
U G / L \\
U G / L \\
U G / L \\
U G / L \\
U G / L \\
\text { UG/L } \\
P C I / L \\
P C I / L \\
P C I / L \\
U G / L \\
U G / L \\
U G / L \\
M G / L \\
M G / L \\
P C I / L \\
P C I / L \\
P C I / L \\
P C I / L \\
U G / L \\
U G / L \\
U G / L \\
P C I / L \\
P C I / L \\
P C I / L \\
P C I / L \\
P C I / L \\
P C I / L \\
M G / L \\
M G / L \\
P C I / L \\
P C I / L \\
P C I / L \\
U G / L \\
U G / L \\
P C I / L \\
P C I / L \\
P C I / L \\
P C I / L \\
U G / L \\
M G / L \\
M G / L \\
M G / L \\
P C I / L \\
P C I / L \\
U G / L\end{array}$ & $\begin{array}{l}\text { EPA } 300.0 \\
\text { EPA } 300.0 \\
\text { EPA } 900.0 \\
\text { EPA } 900.0 \\
\text { EPA } 900.0 \\
\text { EPA } 353.1 \\
\text { EPA } 353.2 \\
\text { EPA } 300.0 \\
\text { EPA } 160.2 \\
\text { EPA } 160.2 \\
\text { ASTM } 5174-91 \\
\text { EPA } 6020 \\
\text { ASTM } 5174-91 \\
\text { EPA } 6010 A \\
\text { EPA } 200.7 \\
\text { EPA } 200.7 \\
\text { EPA } 6010 A \\
\text { EPA } 200.7 \\
\text { EPA } 200.7 \\
\text { EPA } 900.0 \\
\text { EPA } 900.0 \\
\text { EPA } 900.0 \\
\text { EPA } 6010 A \\
\text { EPA } 200.7 \\
\text { EPA } 200.7 \\
\text { EPA } 353.1 \\
\text { EPA } 353.2 \\
\text { EPA } 903.1 \\
\text { EPA } 903.0 \\
\text { EPA } 904.0 \\
\text { EPA } 904.0 \\
\text { EPA } 6010 A \\
\text { EPA } 200.7 \\
\text { EPA } 200.7 \\
\text { HASL } 300 \\
\text { EML TH-01 } \\
\text { HASL } 300 \\
\text { EML } T H-01 \\
\text { HASL } 300 \\
\text { EML TH-01 } \\
\text { EPA } 160.2 \\
\text { EPA } 160.2 \\
\text { ASTM } 5174-91 \\
\text { EPA } 6020 \\
\text { ASTM } 5174-91 \\
\text { EPA } 200.7 \\
\text { EPA } 200.7 \\
\text { EPA } 900.0 \\
\text { EPA } 900.0 \\
\text { EPA } 900.0 \\
\text { EPA } 900.0 \\
\text { EPA } 200.7 \\
\text { EPA } 353.2 \\
\text { EPA } 353.1 \\
\text { EPA } 353.2 \\
\text { EPA } 903.1 \\
\text { EPA } 904.0 \\
\text { EPA } 200.7\end{array}$ \\
\hline
\end{tabular}




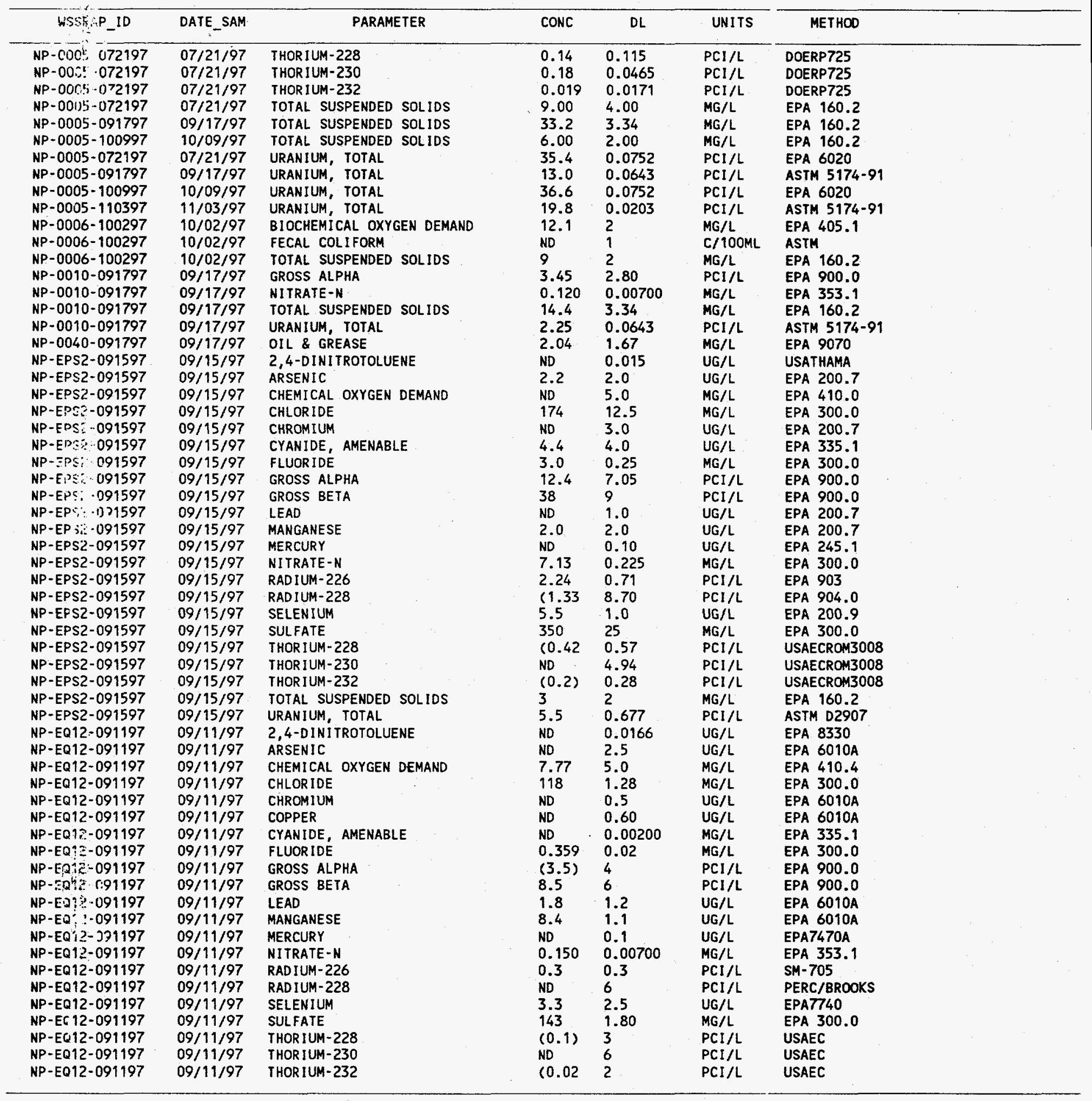




\begin{tabular}{|c|c|c|c|c|c|c|}
\hline WSSRAP_ID & DATE_SAM & PARAMETER & CONC & $D L$ & UNITS & METHOD \\
\hline $\begin{array}{l}\text { NP-EQ12-091197 } \\
\text { NP-EQ12-091197 }\end{array}$ & $\begin{array}{l}09 / 11 / 97 \\
09 / 11 / 97\end{array}$ & $\begin{array}{l}\text { TOTAL SUSPENDED SOLIDS } \\
\text { URANIUM, TOTAL }\end{array}$ & $\begin{array}{l}10.33 \\
0.8\end{array}$ & $\begin{array}{l}2.79 \\
0.677\end{array}$ & $\begin{array}{l}\text { MG/L } \\
\text { PCI/L }\end{array}$ & $\begin{array}{l}\text { EPA } 160.2 \\
\text { ASTM } 02907\end{array}$ \\
\hline
\end{tabular}


GROUNDWATER 


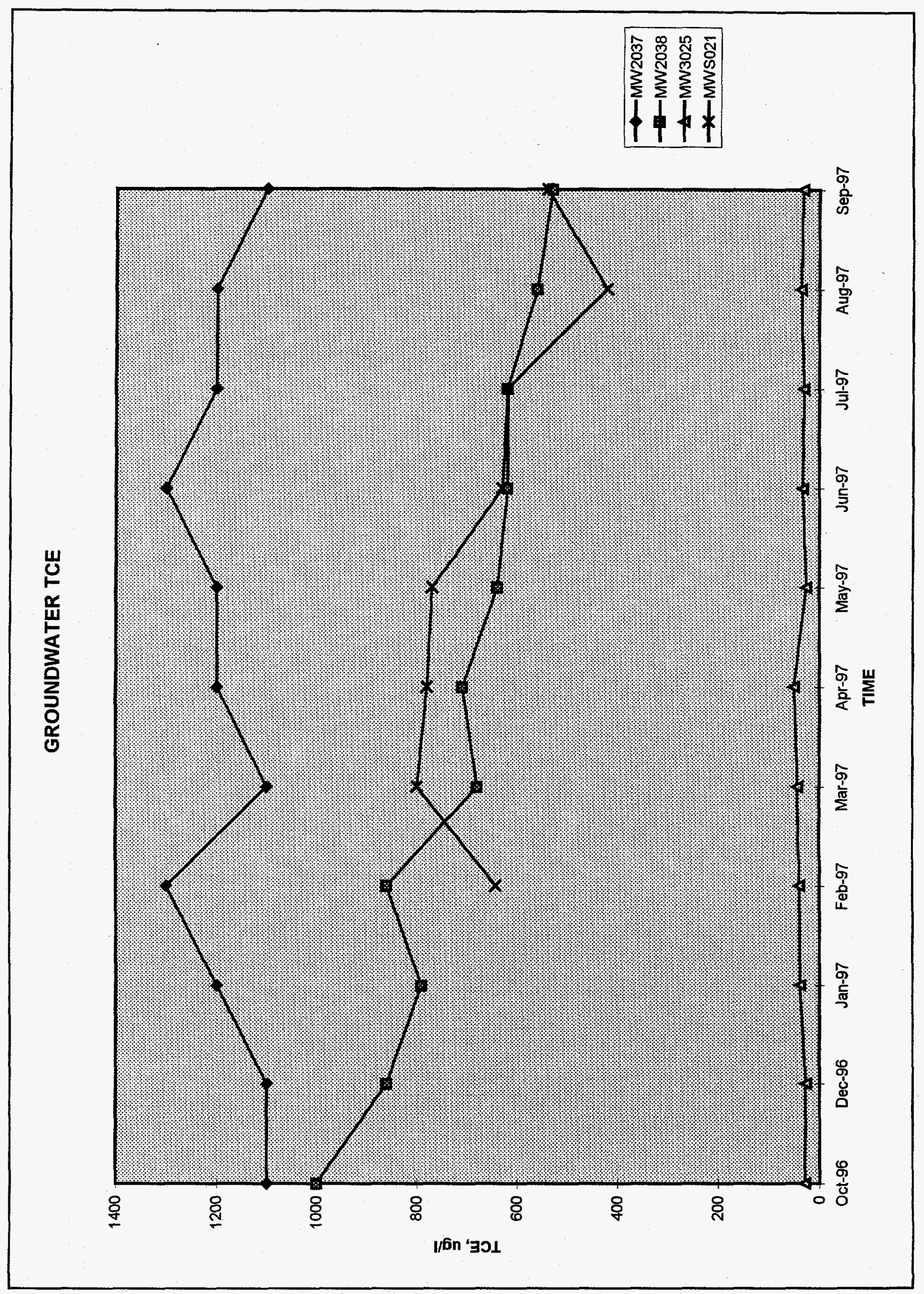




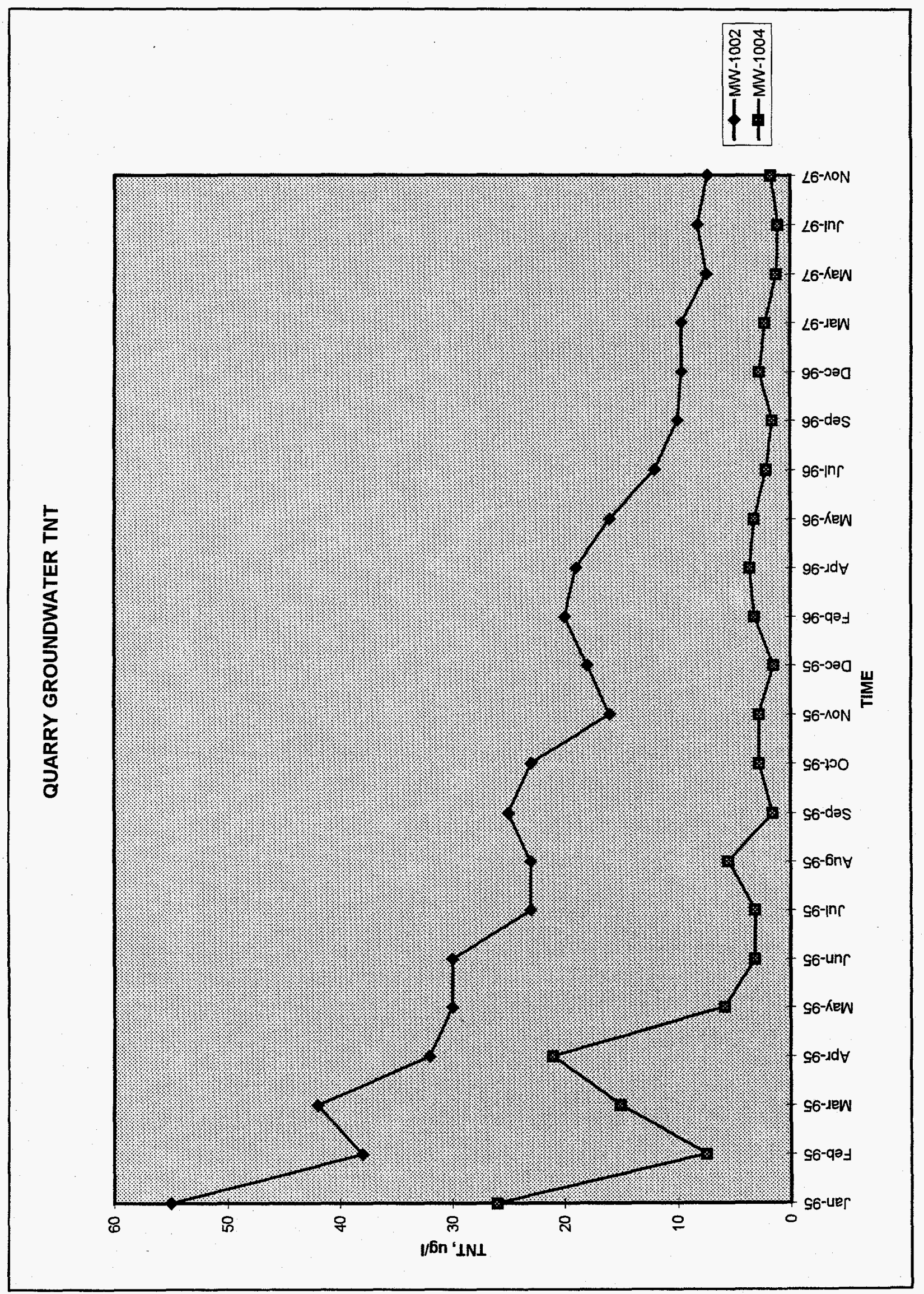




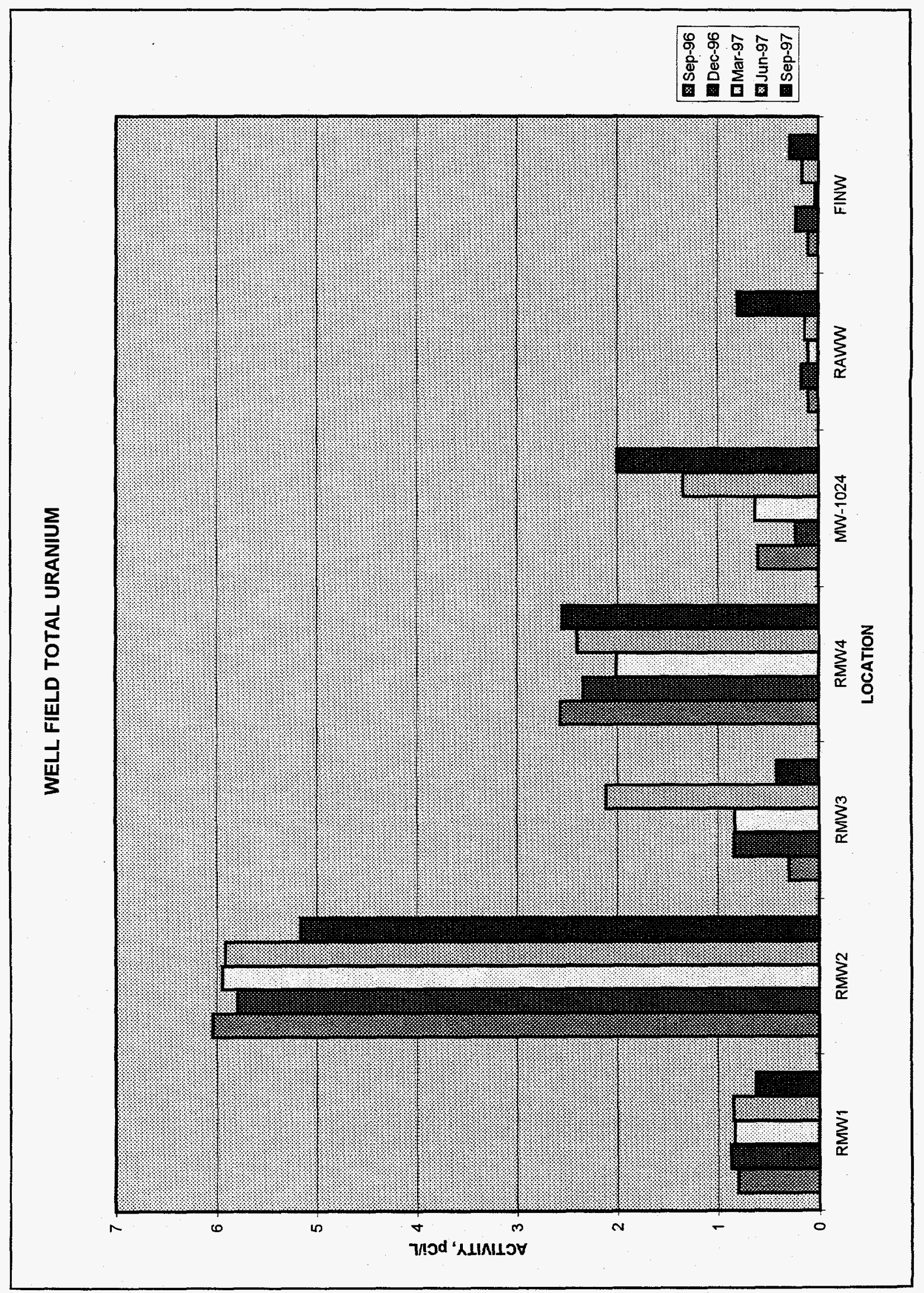


BURGERMEISTER SPRING, LOW FLOW

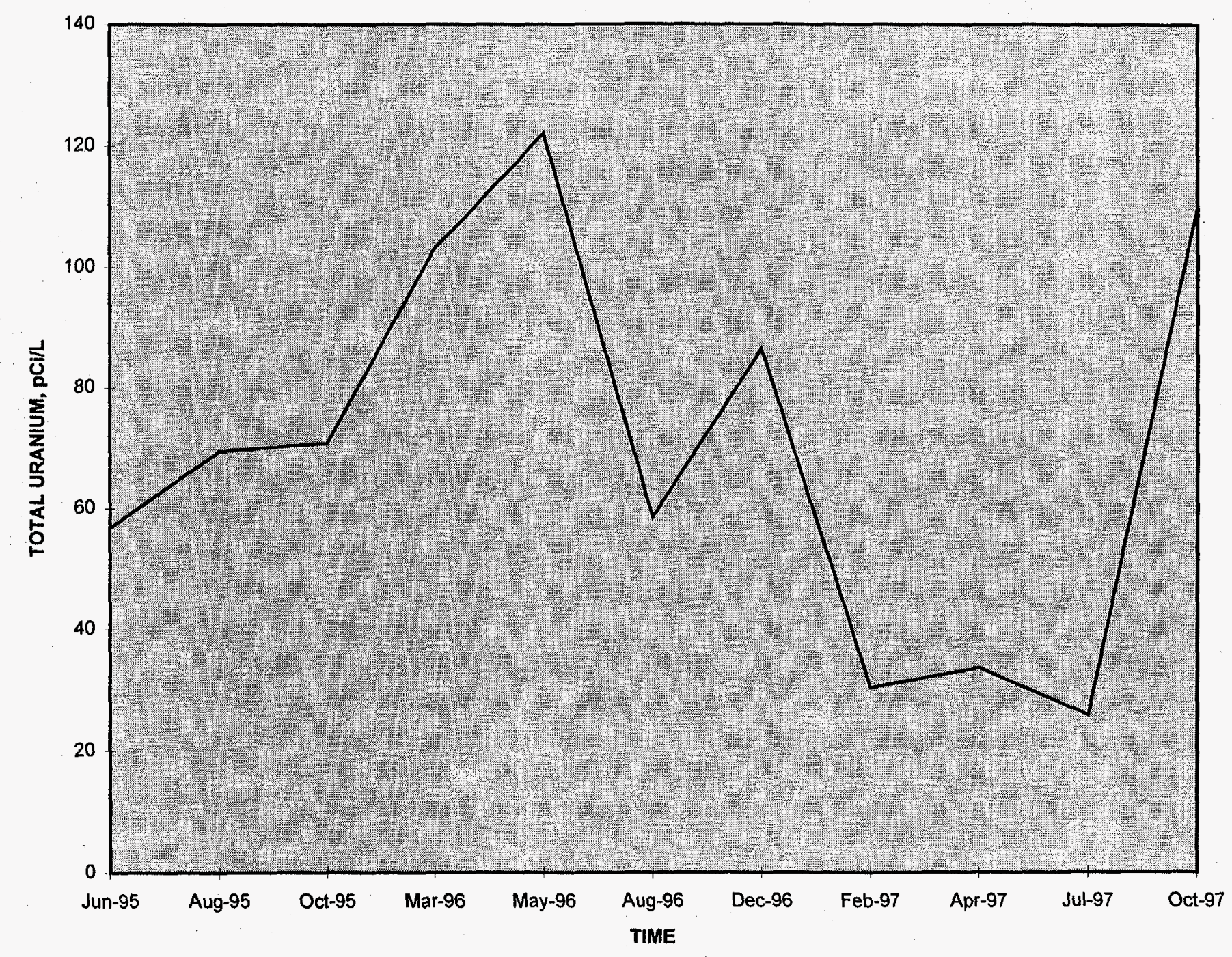

BURGERMEISTER SPRING 
GROUNDWATER

DATA MERGED DURING FOURTH QUARTER 1997

\begin{tabular}{|c|c|c|c|c|c|c|}
\hline WSSRAP_ID & DATE_SAM & PARAMETER & CONC & DL & UNITS & METHOD \\
\hline 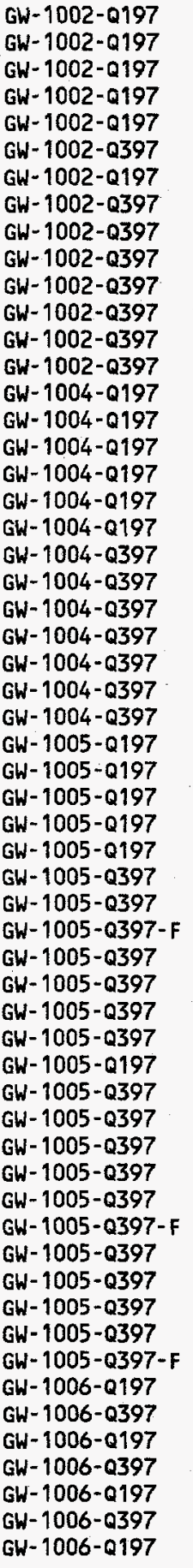 & $\begin{array}{l}03 / 10 / 97 \\
03 / 10 / 97 \\
03 / 10 / 97 \\
03 / 10 / 97 \\
03 / 10 / 97 \\
07 / 14 / 97 \\
03 / 10 / 97 \\
07 / 14 / 97 \\
07 / 14 / 97 \\
07 / 14 / 97 \\
07 / 14 / 97 \\
07 / 14 / 97 \\
07 / 14 / 97 \\
07 / 14 / 97 \\
03 / 10 / 97 \\
03 / 10 / 97 \\
03 / 10 / 97 \\
03 / 10 / 97 \\
03 / 10 / 97 \\
03 / 10 / 97 \\
07 / 14 / 97 \\
07 / 14 / 97 \\
07 / 14 / 97 \\
07 / 14 / 97 \\
07 / 14 / 97 \\
07 / 14 / 97 \\
07 / 14 / 97 \\
03 / 10 / 97 \\
03 / 10 / 97 \\
03 / 10 / 97 \\
03 / 10 / 97 \\
03 / 10 / 97 \\
07 / 14 / 97 \\
07 / 14 / 97 \\
07 / 14 / 97 \\
07 / 14 / 97 \\
07 / 14 / 97 \\
07 / 14 / 97 \\
07 / 14 / 97 \\
03 / 10 / 97 \\
07 / 14 / 97 \\
07 / 14 / 97 \\
07 / 14 / 97 \\
07 / 14 / 97 \\
07 / 14 / 97 \\
07 / 14 / 97 \\
07 / 14 / 97 \\
07 / 14 / 97 \\
07 / 14 / 97 \\
07 / 14 / 97 \\
07 / 14 / 97 \\
02 / 26 / 97 \\
09 / 02 / 97 \\
02 / 26 / 97 \\
09 / 02 / 97 \\
02 / 26 / 97 \\
09 / 02 / 97 \\
02 / 26 / 97\end{array}$ & 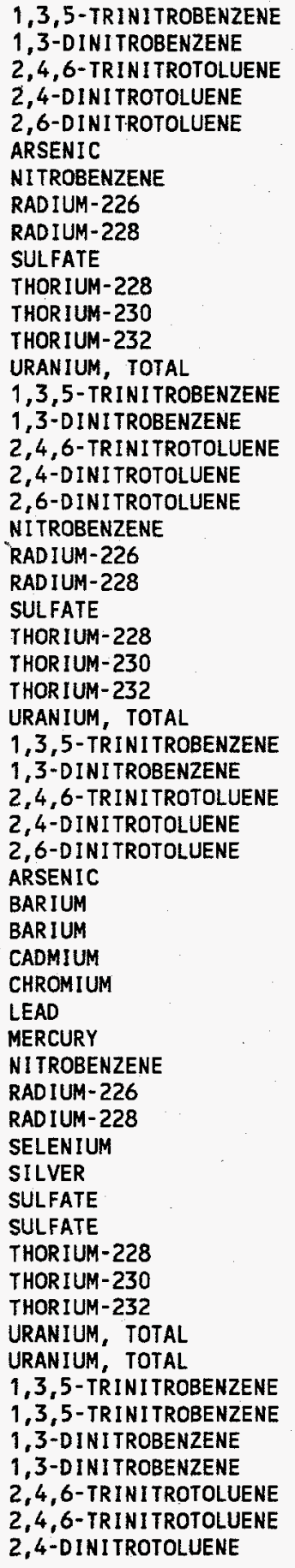 & $\begin{array}{l}36 \\
0.24 \\
9.6 \\
0.077 \\
6.1 \\
\text { ND } \\
\text { ND } \\
0.234 \\
\text { ND } \\
78.4 \\
\text { ND } \\
0.327 \\
\text { ND } \\
4.40 \\
0.72 \\
(0.04 \\
2.2 \\
0.12 \\
0.39 \\
\text { ND } \\
0.328 \\
3.50 \\
132 \\
\text { ND } \\
0.150 \\
10.03 \\
2460 \\
\text { ND } \\
\text { ND } \\
\text { ND } \\
\text { ND } \\
0.017 \\
28.5 \\
1100 \\
51.7 \\
\text { ND } \\
136 \\
170 \\
0.300 \\
\text { ND } \\
1.26 \\
\text { ND } \\
\text { ND } \\
\text { ND } \\
538 \\
534 \\
00.05 \\
0.287 \\
0.073 \\
2430 \\
2250 \\
110 \\
17 \\
0.08 \\
\text { ND } \\
7.3 \\
1.6 \\
0.45\end{array}$ & $\begin{array}{l}0.030 \\
0.090 \\
0.030 \\
0.030 \\
0.010 \\
100 \\
0.030 \\
0 \\
0.061 \\
1.27 \\
2.00 \\
0\end{array}$ & $\begin{array}{l}U G / L \\
U G / L \\
U G / L \\
U G / L \\
U G / L \\
U G / L \\
U G / L \\
P C I / L \\
P C I / L \\
M G / L \\
P C I / L \\
P C I / L \\
P C I / L \\
P C I / L \\
U G / L \\
U G / L \\
U G / L \\
U G / L \\
U G / L \\
U G / L \\
P C I / L \\
P C I / L \\
M G / L \\
P C I / L \\
P C I / L \\
P C I / L \\
P C I / L \\
U G / L \\
U G / L \\
U G / L \\
U G / L \\
U G / L \\
U G / L \\
U G / L \\
U G / L \\
U G / L \\
U G / L \\
U G / L \\
U G / L \\
U G / L \\
P C I / L \\
P C I / L \\
U G / L \\
U G / L \\
M G / L \\
M G / L \\
P C I / L \\
P C I / L \\
P C I / L \\
P C I / L \\
P C I / L \\
U G / L \\
U G / L \\
U G / L \\
U G / L \\
U G / L \\
U G / L \\
U G / L \\
\text { US } \\
\text { U. }\end{array}$ & $\begin{array}{l}\text { USATHAMA } \\
\text { USATHAMA } \\
\text { USATHAMA } \\
\text { USATHAMA } \\
\text { USATHAMA } \\
\text { EPA CLP } \\
\text { USATHAMA } \\
\text { EPA } 903.1 \\
\text { EPA } 904.0 \\
\text { EPA } 300.0 \\
\text { DOERP725 } \\
\text { DOERP725 } \\
\text { DOERP725 } \\
\text { EPA } 6020 \\
\text { USATHAMA } \\
\text { USATHAMA } \\
\text { USATHAMA } \\
\text { USATHAMA } \\
\text { USATHAMA } \\
\text { USATHAMA } \\
\text { EPA } 903.1 \\
\text { EPA } 904.0 \\
\text { EPA } 300.0 \\
\text { DOERP725 } \\
\text { DOERP725 } \\
\text { DOERP725 } \\
\text { EPA } 6020 \\
\text { USATHAMA } \\
\text { USATHAMA } \\
\text { USATHAMA } \\
\text { USATHAMA } \\
\text { USATHAMA } \\
\text { EPA CLP } \\
\text { EPA CLP } \\
\text { EPA CLP } \\
\text { EPA CLP } \\
\text { EPA CLP } \\
\text { EPA CLP } \\
\text { EPA CLP } \\
\text { USATHAMA } \\
\text { EPA } 903.1 \\
\text { EPA } 904.0 \\
\text { EPA CLP } \\
\text { EPA CLP } \\
\text { EPA } 300.0 \\
\text { EPA } 300.0 \\
\text { DOERP725 } \\
\text { DOERP725 } \\
\text { DOERP725 } \\
\text { EPA } 6020 \\
\text { EPA } 6020 \\
\text { USATHAMA } \\
\text { USATHAMA } \\
\text { USATHAMA } \\
\text { USATHAMA } \\
\text { USATHAMA } \\
\text { USATHAMA } \\
\text { USATHAMA }\end{array}$ \\
\hline
\end{tabular}




\begin{tabular}{|c|c|c|c|c|c|c|}
\hline WSSRAP_ID & DATE_SAM & PARAMETER & CONC & $D L$ & UNITS & METHOD \\
\hline 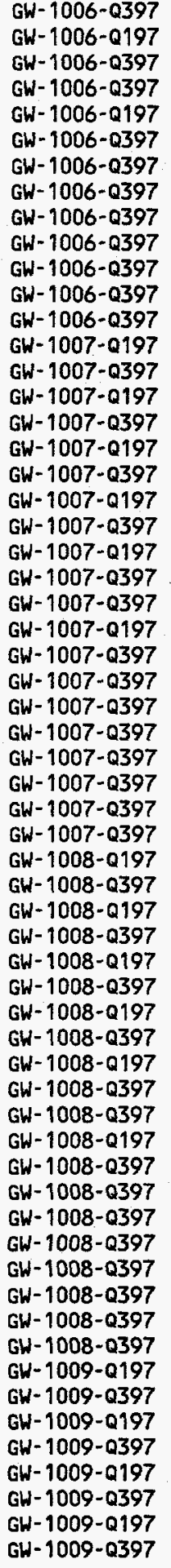 & $\begin{array}{l}09 / 02 / 97 \\
02 / 26 / 97 \\
09 / 02 / 97 \\
09 / 02 / 97 \\
02 / 26 / 97 \\
09 / 02 / 97 \\
09 / 02 / 97 \\
09 / 02 / 97 \\
09 / 02 / 97 \\
09 / 02 / 97 \\
09 / 02 / 97 \\
09 / 02 / 97 \\
09 / 02 / 97 \\
02 / 26 / 97 \\
09 / 02 / 97 \\
02 / 26 / 97 \\
09 / 02 / 97 \\
02 / 26 / 97 \\
09 / 02 / 97 \\
02 / 26 / 97 \\
09 / 02 / 97 \\
02 / 26 / 97 \\
09 / 02 / 97 \\
09 / 02 / 97 \\
02 / 26 / 97 \\
09 / 02 / 97 \\
09 / 02 / 97 \\
09 / 02 / 97 \\
09 / 02 / 97 \\
09 / 02 / 97 \\
09 / 02 / 97 \\
09 / 02 / 97 \\
09 / 02 / 97 \\
02 / 26 / 97 \\
09 / 02 / 97 \\
02 / 26 / 97 \\
09 / 02 / 97 \\
02 / 26 / 97 \\
09 / 02 / 97 \\
02 / 26 / 97 \\
09 / 02 / 97 \\
02 / 26 / 97 \\
09 / 02 / 97 \\
09 / 02 / 97 \\
02 / 26 / 97 \\
09 / 02 / 97 \\
09 / 02 / 97 \\
09 / 02 / 97 \\
09 / 02 / 97 \\
09 / 02 / 97 \\
09 / 02 / 97 \\
09 / 02 / 97 \\
09 / 02 / 97 \\
02 / 26 / 97 \\
09 / 02 / 97 \\
02 / 26 / 97 \\
09 / 02 / 97 \\
02 / 26 / 97 \\
09 / 02 / 97 \\
02 / 26 / 97 \\
09 / 02 / 97\end{array}$ & 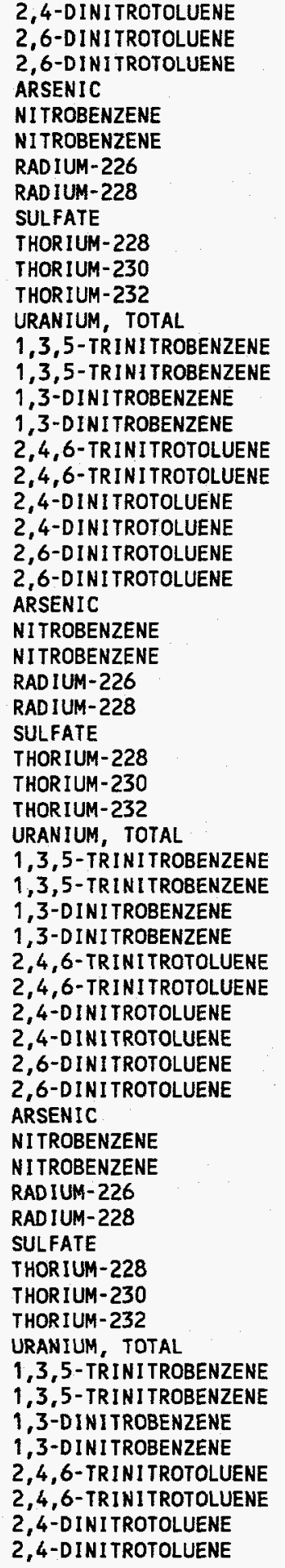 & 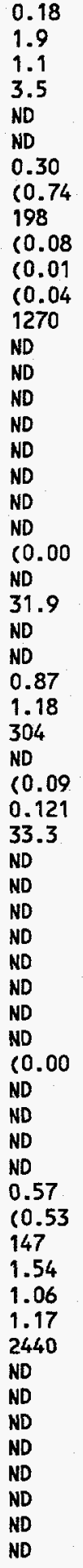 & $\begin{array}{l}0.030 \\
0.010 \\
0.010 \\
3.0 \\
0.030 \\
0.030 \\
0.28 \\
0.86 \\
0.10 \\
0.27 \\
0.094 \\
0.10 \\
4.2 \\
0.030 \\
0.030 \\
0.090 \\
0.090 \\
0.030 \\
0.030 \\
0.030 \\
0.030 \\
0.010 \\
0.01 \\
3.0 \\
0.030 \\
0.030 \\
0.20 \\
0.86 \\
0.10 \\
0.26 \\
0.10 \\
0.085 \\
4.2 \\
0.030 \\
0.030 \\
0.090 \\
0.090 \\
0.030 \\
0.030 \\
0.030 \\
0.030 \\
0.010 \\
0.010 \\
3.0 \\
0.030 \\
0.030 \\
0.31 \\
0.73 \\
0.10 \\
0.25 \\
0.13 \\
0.10 \\
8.4 \\
0.030 \\
0.030 \\
0.090 \\
0.090 \\
0.030 \\
0.030 \\
0.030 \\
0.030\end{array}$ & $\begin{array}{l}U G / L \\
U G / L \\
U G / L \\
U G / L \\
U G / L \\
U G / L \\
P C I / L \\
P C I / L \\
M G / L \\
P C I / L \\
P C I / L \\
P C I / L \\
P C I / L \\
U G / L \\
U G / L \\
U G / L \\
U G / L \\
U G / L \\
U G / L \\
U G / L \\
U G / L \\
U G / L \\
U G / L \\
U G / L \\
U G / L \\
U G / L \\
P C I / L \\
P C I / L \\
M G / L \\
P C I / L \\
P C I / L \\
P C I / L \\
P C I / L \\
U G / L \\
U G / L \\
U G / L \\
U G / L \\
U G / L \\
U G / L \\
U G / L \\
U G / L \\
U G / L \\
U G / L \\
U G / L \\
U G / L \\
U G / L \\
P C I / L \\
P C I / L \\
M G / L \\
P C I / L \\
P C I / L \\
P C I / L \\
P C I / L \\
U G / L \\
U G / L \\
U G / L \\
U G / L \\
U G / L \\
U G / L \\
U G / L \\
U G / L \\
\end{array}$ & 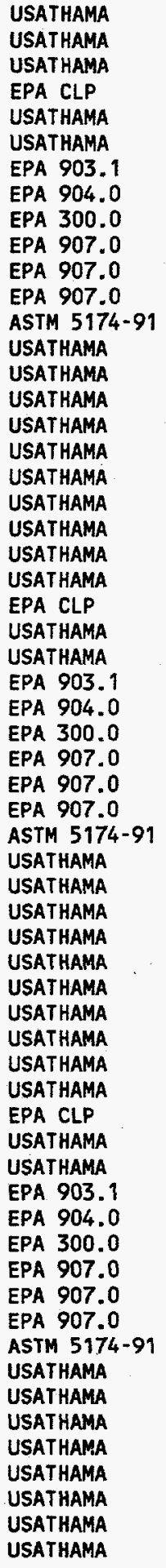 \\
\hline
\end{tabular}




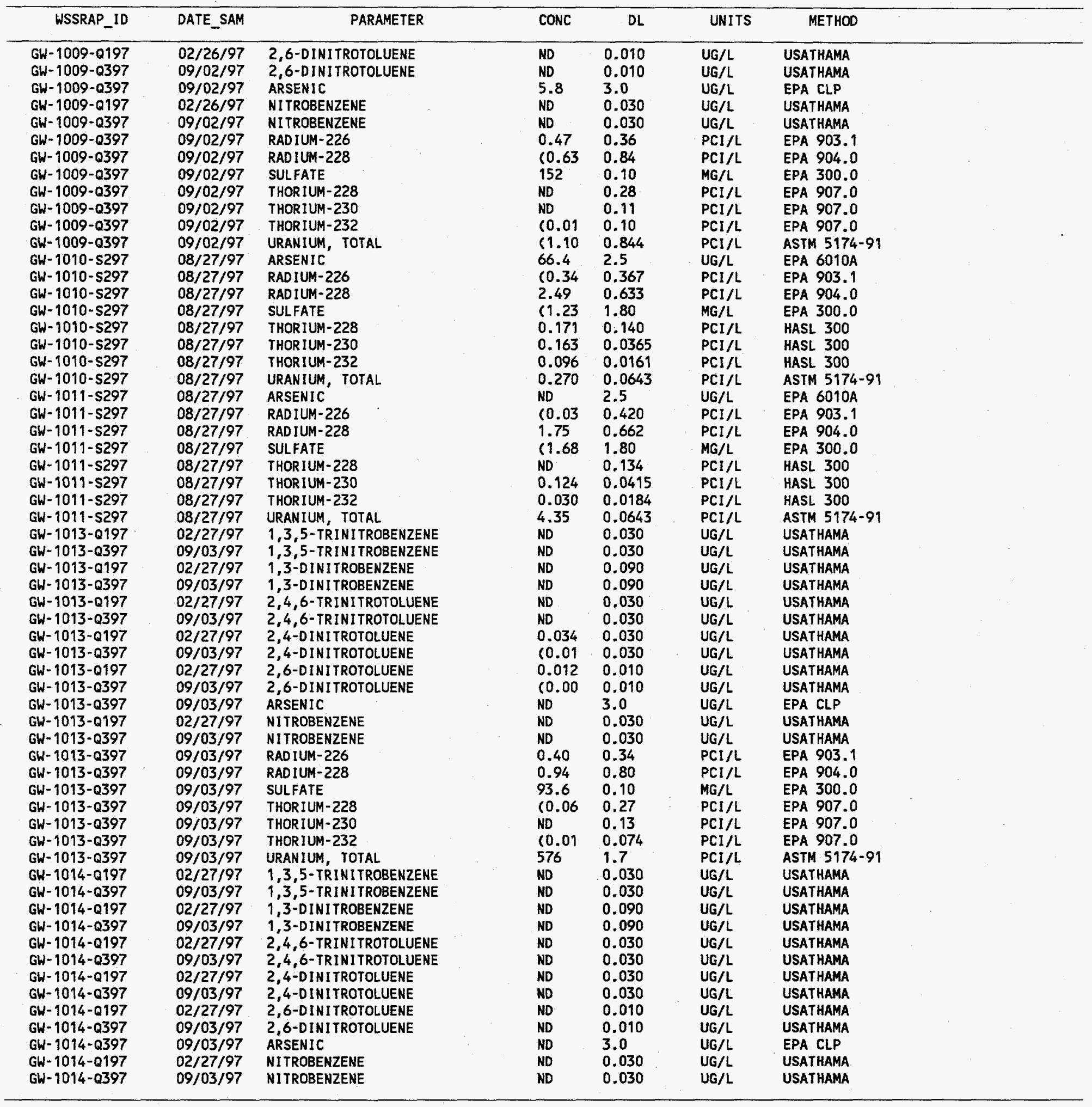




\begin{tabular}{|c|c|c|c|c|c|c|}
\hline WSSRAP_ID & DATE_SAM & PARAMETER & CONC & $D L$ & UNITS & METHOD \\
\hline 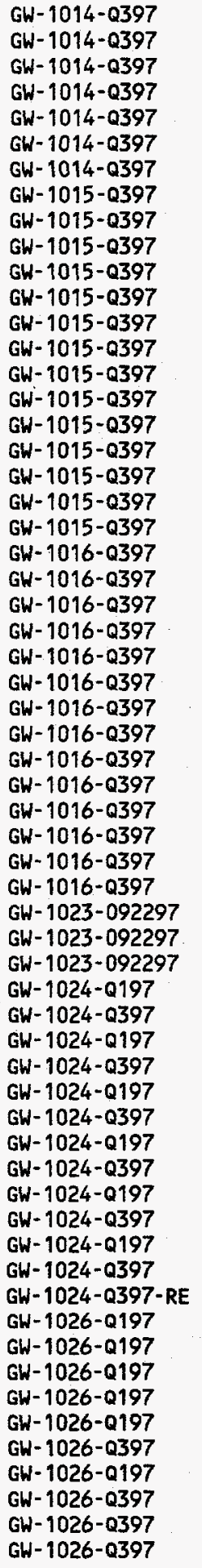 & $\begin{array}{l}09 / 03 / 97 \\
09 / 03 / 97 \\
09 / 03 / 97 \\
09 / 03 / 97 \\
09 / 03 / 97 \\
09 / 03 / 97 \\
09 / 03 / 97 \\
09 / 04 / 97 \\
09 / 04 / 97 \\
09 / 04 / 97 \\
09 / 04 / 97 \\
09 / 04 / 97 \\
09 / 04 / 97 \\
09 / 04 / 97 \\
09 / 04 / 97 \\
09 / 04 / 97 \\
09 / 04 / 97 \\
09 / 04 / 97 \\
09 / 04 / 97 \\
09 / 04 / 97 \\
09 / 04 / 97 \\
09 / 04 / 97 \\
09 / 04 / 97 \\
09 / 04 / 97 \\
09 / 04 / 97 \\
09 / 04 / 97 \\
09 / 04 / 97 \\
09 / 04 / 97 \\
09 / 04 / 97 \\
09 / 04 / 97 \\
09 / 04 / 97 \\
09 / 04 / 97 \\
09 / 04 / 97 \\
09 / 04 / 97 \\
09 / 04 / 97 \\
09 / 22 / 97 \\
09 / 22 / 97 \\
09 / 22 / 97 \\
02 / 27 / 97 \\
09 / 09 / 97 \\
02 / 27 / 97 \\
09 / 09 / 97 \\
02 / 27 / 97 \\
09 / 09 / 97 \\
02 / 27 / 97 \\
09 / 09 / 97 \\
02 / 27 / 97 \\
09 / 09 / 97 \\
02 / 27 / 97 \\
09 / 09 / 97 \\
09 / 09 / 97 \\
03 / 04 / 97 \\
03 / 04 / 97 \\
03 / 04 / 97 \\
03 / 04 / 97 \\
03 / 04 / 97 \\
08 / 07 / 97 \\
03 / 04 / 97 \\
08 / 07 / 97 \\
08 / 07 / 97 \\
08 / 07 / 97\end{array}$ & 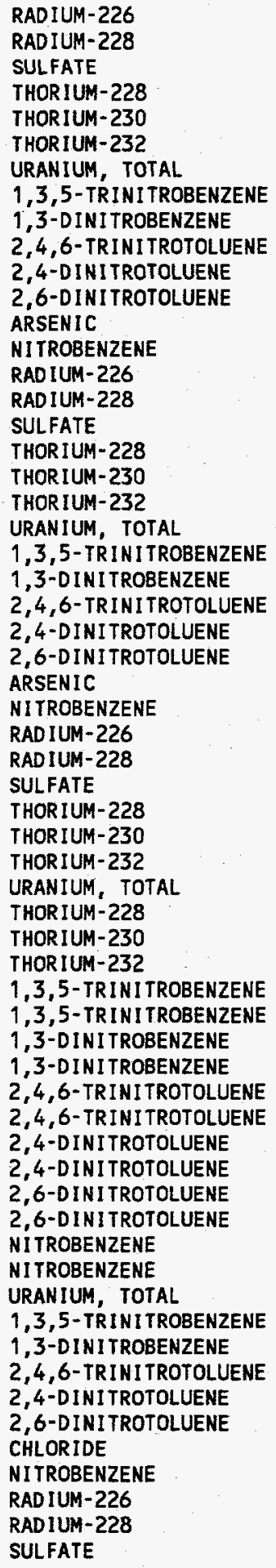 & $\begin{array}{l}\text { C.16 } \\
\text { C.61 } \\
95.1 \\
C 0.07 \\
C 0.02 \\
C 0.01 \\
708 \\
3.2 \\
\text { ND } \\
1.4 \\
C 0.02 \\
0.16 \\
2.6 \\
\text { ND } \\
0.807 \\
1.10 \\
126 \\
0.282 \\
0.136 \\
C 0.04 \\
273 \\
0.070 \\
\text { ND } \\
0.047 \\
\text { ND } \\
0.023 \\
\text { ND } \\
\text { ND } \\
0.529 \\
2.25 \\
144 \\
0.189 \\
0.168 \\
0.082 \\
168 \\
0.140 \\
0.260 \\
0.120 \\
\text { ND } \\
\text { ND } \\
\text { ND } \\
\text { ND } \\
\text { ND } \\
\text { ND } \\
\text { ND } \\
\text { ND } \\
\text { ND } \\
\text { ND } \\
\text { ND } \\
\text { ND } \\
2.0 \\
\text { ND } \\
\text { ND } \\
\text { ND } \\
\text { ND } \\
\text { ND } \\
2.92 \\
\text { ND } \\
0.694 \\
1.21 \\
2.68\end{array}$ & 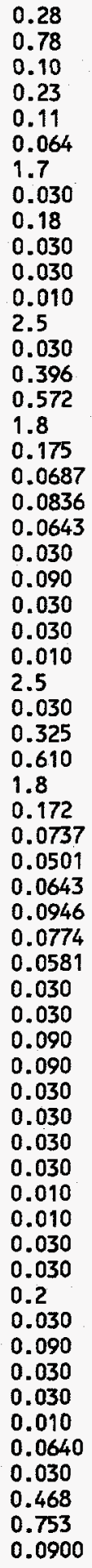 & 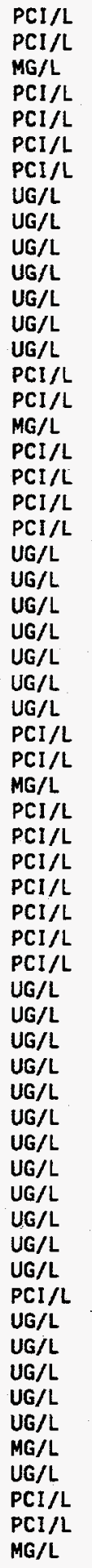 & $\begin{array}{l}\text { EPA } 903.1 \\
\text { EPA } 904.0 \\
\text { EPA } 300.0 \\
\text { EPA 907.0 } \\
\text { EPA 907.0 } \\
\text { EPA } 907.0 \\
\text { ASTM } 5174-91 \\
\text { USATHAMA } \\
\text { USATHAMA } \\
\text { USATHAMA } \\
\text { USATHAMA } \\
\text { USATHAMA } \\
\text { EPA } 6010 A \\
\text { USATHAMA } \\
\text { EPA 903.1 } \\
\text { EPA 904.0 } \\
\text { EPA } 300.0 \\
\text { HASL } 300 \\
\text { HASL } 300 \\
\text { HASL } 300 \\
\text { ASTM } 5174-91 \\
\text { USATHAMA } \\
\text { USATHAMA } \\
\text { USATHAMA } \\
\text { USATHAMA } \\
\text { USATHAMA } \\
\text { EPA } 6010 A \\
\text { USATHAMA } \\
\text { EPA 903.1 } \\
\text { EPA 904.0 } \\
\text { EPA } 300.0 \\
\text { HASL } 300 \\
\text { HASL } 300 \\
\text { HASL } 300 \\
\text { ASTM } 5174-91 \\
\text { DOERP725 } \\
\text { DOERP725 } \\
\text { DOERP725 } \\
\text { USATHAMA } \\
\text { USATHAMA } \\
\text { USATHAMA } \\
\text { USATHAMA } \\
\text { USATHAMA } \\
\text { USATHAMA } \\
\text { USATHAMA } \\
\text { USATHAMA } \\
\text { USATHAMA } \\
\text { USATHAMA } \\
\text { USATHAMA } \\
\text { USATHAMA } \\
\text { ASTM D2907 } \\
\text { USATHAMA } \\
\text { USATHAMA } \\
\text { USATHAMA } \\
\text { USATHAMA } \\
\text { USATHAMA } \\
\text { EPA } 300.0 \\
\text { USATHAMA } \\
\text { EPA } 903.1 \\
\text { EPA 904.0 } \\
\text { EPA } 300.0 \\
\end{array}$ \\
\hline
\end{tabular}




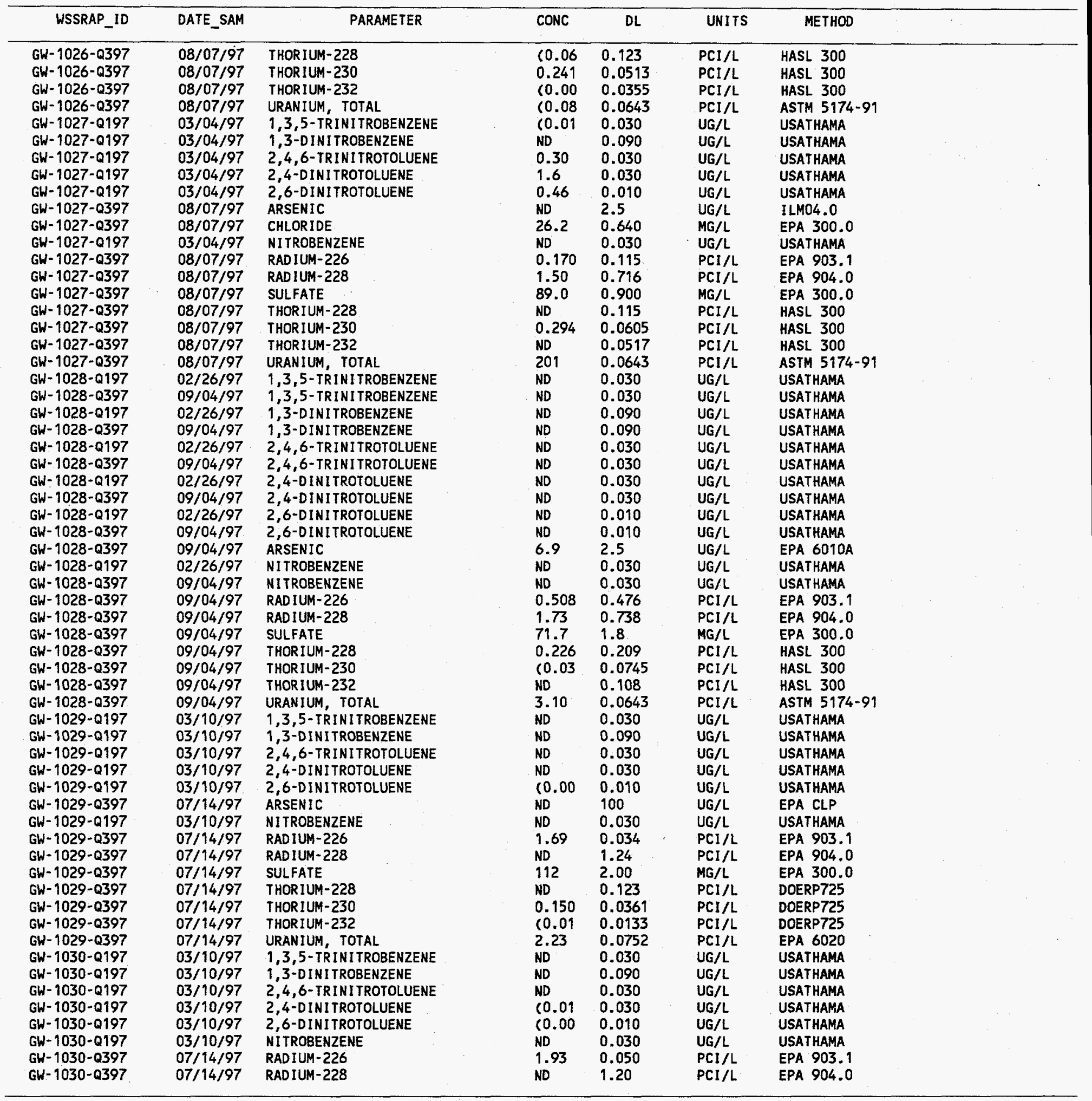




\begin{tabular}{|c|c|c|c|c|c|c|c|}
\hline WSSRAP_ID & DATE_SAM & PARAMETER & CONC & $\mathrm{DL}$ & UNITS & METHOD & \\
\hline 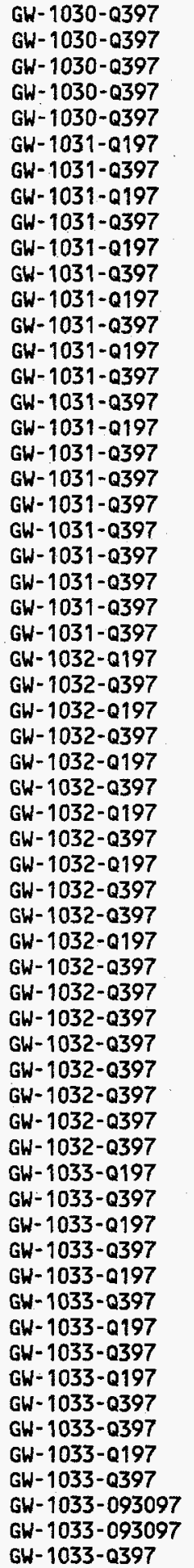 & $\begin{array}{l}07 / 14 / 97 \\
07 / 14 / 97 \\
07 / 14 / 97 \\
07 / 14 / 97 \\
07 / 14 / 97 \\
02 / 27 / 97 \\
09 / 03 / 97 \\
02 / 27 / 97 \\
09 / 03 / 97 \\
02 / 27 / 97 \\
09 / 03 / 97 \\
02 / 27 / 97 \\
09 / 03 / 97 \\
02 / 27 / 97 \\
09 / 03 / 97 \\
09 / 03 / 97 \\
02 / 27 / 97 \\
09 / 03 / 97 \\
09 / 03 / 97 \\
09 / 03 / 97 \\
09 / 03 / 97 \\
09 / 03 / 97 \\
09 / 03 / 97 \\
09 / 03 / 97 \\
09 / 03 / 97 \\
02 / 26 / 97 \\
09 / 03 / 97 \\
02 / 26 / 97 \\
09 / 03 / 97 \\
02 / 26 / 97 \\
09 / 03 / 97 \\
02 / 26 / 97 \\
09 / 03 / 97 \\
02 / 26 / 97 \\
09 / 03 / 97 \\
09 / 03 / 97 \\
02 / 26 / 97 \\
09 / 03 / 97 \\
09 / 03 / 97 \\
09 / 03 / 97 \\
09 / 03 / 97 \\
09 / 03 / 97 \\
09 / 03 / 97 \\
09 / 03 / 97 \\
09 / 03 / 97 \\
03 / 06 / 97 \\
09 / 16 / 97 \\
03 / 06 / 97 \\
09 / 16 / 97 \\
03 / 06 / 97 \\
09 / 16 / 97 \\
03 / 06 / 97 \\
09 / 16 / 97 \\
03 / 06 / 97 \\
09 / 16 / 97 \\
09 / 16 / 97 \\
03 / 06 / 97 \\
09 / 16 / 97 \\
09 / 30 / 97 \\
09 / 30 / 97 \\
09 / 16 / 97\end{array}$ & 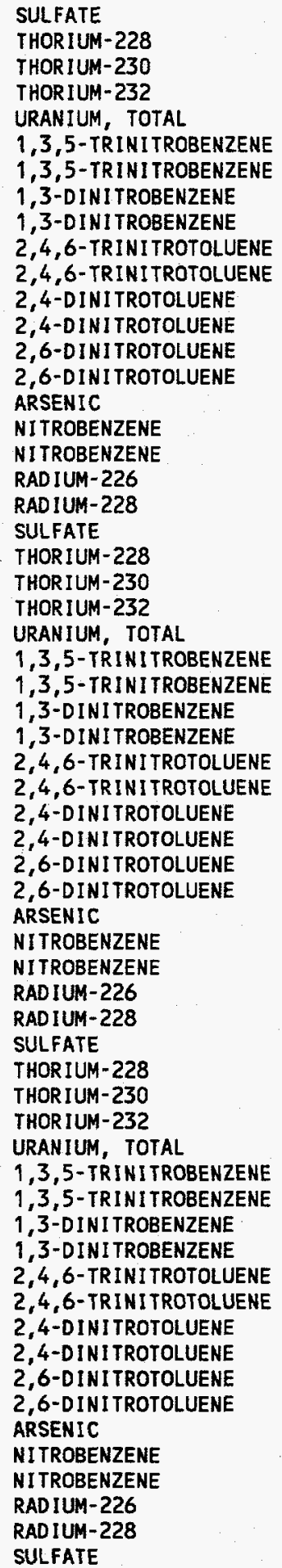 & $\begin{array}{l}81.2 \\
\text { C0.04 } \\
0.363 \\
0.104 \\
27.8 \\
\text { ND } \\
\text { ND } \\
\text { ND } \\
\text { ND } \\
\text { ND } \\
\text { ND } \\
\text { ND } \\
\text { ND } \\
\text { ND } \\
\text { ND } \\
\text { ND } \\
\text { ND } \\
\text { ND } \\
0.34 \\
0.73 \\
70.6 \\
\text { ND } \\
\text { C.02 } \\
\text { ND } \\
118 \\
\text { ND } \\
\text { ND } \\
\text { ND } \\
\text { ND } \\
\text { ND } \\
\text { ND } \\
\text { N.02 } \\
\text { ND } \\
0.016 \\
\text { C.00 } \\
\text { ND } \\
\text { ND } \\
\text { ND } \\
0.24 \\
1.19 \\
222 \\
\text { ND } \\
\text { D.00 } \\
\text { C0.01 } \\
1080 \\
\text { ND } \\
\text { ND } \\
\text { ND } \\
\text { ND } \\
\text { ND } \\
\text { ND } \\
\text { ND } \\
\text { ND } \\
\text { ND } \\
\text { ND } \\
\text { ND } \\
\text { ND } \\
\text { ND } \\
0.69 \\
1.09 \\
3.33\end{array}$ & 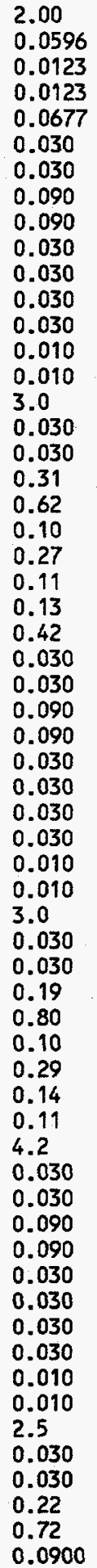 & $\begin{array}{l}M G / L \\
P C I / L \\
P C I / L \\
P C I / L \\
P C I / L \\
U G / L \\
U G / L \\
U G / L \\
U G / L \\
U G / L \\
U G / L \\
U G / L \\
U G / L \\
U G / L \\
U G / L \\
U G / L \\
U G / L \\
U G / L \\
P C I / L \\
P C I / L \\
M G / L \\
P C I / L \\
P C I / L \\
P C I / L \\
P C I / L \\
U G / L \\
U G / L \\
U G / L \\
U G / L \\
U G / L \\
U G / L \\
U G / L \\
U G / L \\
U G / L \\
U G / L \\
U G / L \\
U G / L \\
U G / L \\
P C I / L \\
P C I / L \\
M G / L \\
P C I / L \\
P C I / L \\
P C I / L \\
P C I / L \\
U G / L \\
U G / L \\
U G / L \\
U G / L \\
U G / L \\
U G / L \\
U G / L \\
U G / L \\
U G / L \\
U G / L \\
U G / L \\
\text { UG/L} \\
\text { UG/L } \\
P C I / L \\
P C I / L \\
M G / L\end{array}$ & $\begin{array}{l}\text { EPA } 300.0 \\
\text { DOERP725 } \\
\text { DOERP725 } \\
\text { DOERP725 } \\
\text { EPA } 6020 \\
\text { USATHAMA } \\
\text { USATHAMA } \\
\text { USATHAMA } \\
\text { USATHAMA } \\
\text { USATHAMA } \\
\text { USATHAMA } \\
\text { USATHAMA } \\
\text { USATHAMA } \\
\text { USATHAMA } \\
\text { USATHAMA } \\
\text { EPA CLP } \\
\text { USATHAMA } \\
\text { USATHAMA } \\
\text { EPA } 903.1 \\
\text { EPA } 904.0 \\
\text { EPA } 300.0 \\
\text { EPA } 907.0 \\
\text { EPA } 907.0 \\
\text { EPA } 907.0 \\
\text { ASTM } 5174-91 \\
\text { USATHAMA } \\
\text { USATHAMA } \\
\text { USATHAMA } \\
\text { USATHAMA } \\
\text { USATHAMA } \\
\text { USATHAMA } \\
\text { USATHAMA } \\
\text { USATHAMA } \\
\text { USATHAMA } \\
\text { USATHAMA } \\
\text { EPA CLP } \\
\text { USATHAMA } \\
\text { USATHAMA } \\
\text { EPA } 903.1 \\
\text { EPA } 904.0 \\
\text { EPA } 300.0 \\
\text { EPA } 907.0 \\
\text { EPA } 907.0 \\
\text { EPA } 907.0 \\
\text { ASTM } 5174-91 \\
\text { USATHAMA } \\
\text { USATHAMA } \\
\text { USATHAMA } \\
\text { USATHAMA } \\
\text { USATHAMA } \\
\text { USATHAMA } \\
\text { USATHAMA } \\
\text { USATHAMA } \\
\text { USATHAMA } \\
\text { USATHAMA } \\
\text { EPA } 6010 A \\
\text { USATHAMA } \\
\text { USATHAMA } \\
\text { EPA } 903.1 \\
\text { EPA } 904.0 \\
\text { EPA } 300.0 \\
\end{array}$ & $*$ \\
\hline
\end{tabular}




\begin{tabular}{|c|c|c|c|c|c|c|}
\hline WSSRAP_ID & DATE_SAM & PARAMETER & CONC & $\mathrm{DL}$ & UNITS & METHOD \\
\hline 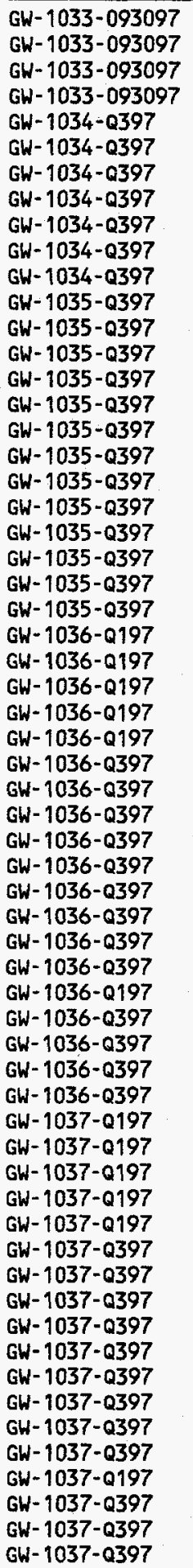 & $\begin{array}{l}09 / 30 / 97 \\
09 / 30 / 97 \\
09 / 30 / 97 \\
09 / 30 / 97 \\
08 / 07 / 97 \\
08 / 07 / 97 \\
08 / 07 / 97 \\
08 / 07 / 97 \\
08 / 07 / 97 \\
08 / 07 / 97 \\
08 / 07 / 97 \\
08 / 111 / 97 \\
08 / 11 / 97 \\
08 / 11 / 97 \\
08 / 11 / 97 \\
08 / 111 / 97 \\
08 / 111 / 97 \\
08 / 111 / 97 \\
08 / 11 / 97 \\
08 / 11 / 97 \\
08 / 11 / 97 \\
08 / 111 / 97 \\
08 / 111 / 97 \\
08 / 11 / 97 \\
03 / 05 / 97 \\
03 / 05 / 97 \\
03 / 05 / 97 \\
03 / 05 / 97 \\
03 / 05 / 97 \\
08 / 11 / 97 \\
08 / 11 / 97 \\
08 / 111 / 97 \\
08 / 11 / 97 \\
08 / 11 / 97 \\
08 / 11 / 97 \\
08 / 11 / 97 \\
08 / 11 / 97 \\
08 / 11 / 97 \\
03 / 05 / 97 \\
08 / 11 / 97 \\
08 / 11 / 97 \\
08 / 11197 \\
08 / 111 / 97 \\
03 / 05 / 97 \\
03 / 05 / 97 \\
03 / 05 / 97 \\
03 / 05 / 97 \\
03 / 05 / 97 \\
08 / 11 / 97 \\
08 / 11 / 97 \\
08 / 111 / 97 \\
08 / 111 / 97 \\
08 / 11 / 97 \\
08 / 11 / 97 \\
08 / 11 / 97 \\
08 / 111 / 97 \\
08 / 111 / 97 \\
03 / 05 / 97 \\
08 / 11 / 97 \\
08 / 11 / 97 \\
08 / 111 / 97\end{array}$ & 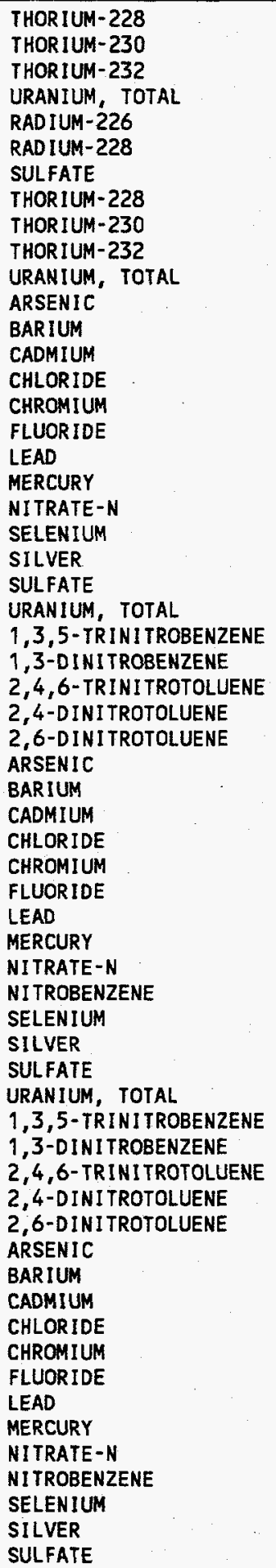 & $\begin{array}{l}\text { CO.09 } \\
\text { ND } \\
\text { C0.05 } \\
2.22 \\
60.40 \\
2.81 \\
52.5 \\
\text { ND } \\
0.177 \\
60.02 \\
2.25 \\
\text { ND } \\
285 \\
\text { ND } \\
25.8 \\
1.8 \\
0.677 \\
\text { ND } \\
\text { ND } \\
0.650 \\
\text { ND } \\
2.6 \\
46.0 \\
0.410 \\
\text { ND } \\
\text { ND } \\
\text { ND } \\
\text { ND } \\
\text { ND } \\
3.8 \\
144 \\
\text { ND } \\
117 \\
3.4 \\
1.12 \\
\text { ND } \\
\text { ND } \\
8.70 \\
\text { ND } \\
\text { ND } \\
2.0 \\
64.7 \\
6.46 \\
\text { ND } \\
\text { ND } \\
\text { ND } \\
\text { ND } \\
\text { ND } \\
5.9 \\
433 \\
0.57 \\
4.32 \\
5.7 \\
0.803 \\
15.6 \\
\text { ND } \\
0.890 \\
\text { ND } \\
\text { ND } \\
1.7 \\
63.7\end{array}$ & $\begin{array}{l}0.24 \\
0.13 \\
0.10 \\
0.42 \\
0.495 \\
0.685 \\
1.80 \\
0.0826 \\
0.0376 \\
0.0293 \\
0.0643 \\
2.5 \\
0.2 \\
0.3 \\
0.640 \\
0.5 \\
0.0200 \\
1.2 \\
0.1 \\
0.00700 \\
2.3 \\
0.6 \\
0.900 \\
0.0643 \\
0.030 \\
0.090 \\
0.030 \\
0.030 \\
0.010 \\
2.5 \\
0.2 \\
0.3 \\
1.28 \\
0.5 \\
0.0200 \\
1.2 \\
0.1 \\
0.0350 \\
0.030 \\
2.3 \\
0.6 \\
1.80 \\
0.0643 \\
0.030 \\
0.090 \\
0.030 \\
0.030 \\
0.010 \\
2.5 \\
0.2 \\
0.3 \\
0.0640 \\
0.5 \\
0.0200 \\
1.2 \\
0.1 \\
0.00700 \\
0.030 \\
2.3 \\
0.6 \\
0.900 \\
\end{array}$ & 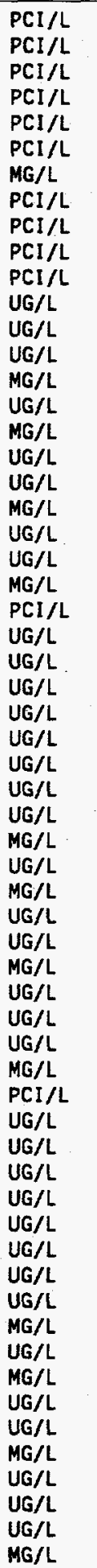 & $\begin{array}{l}\text { EPA } 907.0 \\
\text { EPA } 907.0 \\
\text { EPA } 907.0 \\
\text { ASTM } 5174-91 \\
\text { EPA } 903.1 \\
\text { EPA } 904.0 \\
\text { EPA } 300.0 \\
\text { HASL } 300 \\
\text { HASL } 300 \\
\text { HASL } 300 \\
\text { ASTM } 5174-91 \\
\text { EPA CLP } \\
\text { EPA CLP } \\
\text { EPA CLP } \\
\text { EPA } 300.0 \\
\text { EPA CLP } \\
\text { EPA } 300.0 \\
\text { EPA CLP } \\
\text { EPA CLP } \\
\text { EPA } 353.1 \\
\text { EPA CLP } \\
\text { EPA CLP } \\
\text { EPA } 300.0 \\
\text { ASTM } 5174-91 \\
\text { USATHAMA } \\
\text { USATHAMA } \\
\text { USATHAMA } \\
\text { USATHAMA } \\
\text { USATHAMA } \\
\text { EPA CLP } \\
\text { EPA CLP } \\
\text { EPA CLP } \\
\text { EPA } 300.0 \\
\text { EPA CLP } \\
\text { EPA } 300.0 \\
\text { EPA CLP } \\
\text { EPA CLP } \\
\text { EPA } 353.1 \\
\text { USATHAMA } \\
\text { EPA CLP } \\
\text { EPA CLP } \\
\text { EPA } 300.0 \\
\text { ASTM } 5174-91 \\
\text { USATHAMA } \\
\text { USATHAMA } \\
\text { USATHAMA } \\
\text { USATHAMA } \\
\text { USATHAMA } \\
\text { EPA CLP } \\
\text { EPA CLP } \\
\text { EPA CLP } \\
\text { EPA } 300.0 \\
\text { EPA CLP } \\
\text { EPA } 300.0 \\
\text { EPA CLP } \\
\text { EPA CLP } \\
\text { EPA } 353.1 \\
\text { USATHAMA } \\
\text { EPA CLP } \\
\text { EPA CLP } \\
\text { EPA } 300.0\end{array}$ \\
\hline
\end{tabular}




\begin{tabular}{|c|c|c|c|c|c|c|}
\hline WSSRAP_ID & DATE_SAM & PARAMETER & CONC & $D L$ & UNITS & METHOD \\
\hline 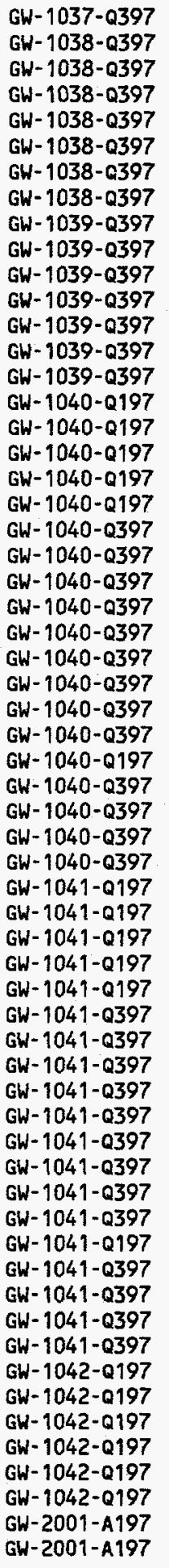 & $\begin{array}{l}08 / 11 / 97 \\
08 / 11 / 97 \\
08 / 11 / 97 \\
08 / 11 / 97 \\
08 / 11 / 97 \\
08 / 11 / 97 \\
08 / 11 / 97 \\
08 / 11 / 97 \\
08 / 11 / 97 \\
08 / 11 / 97 \\
08 / 11 / 97 \\
08 / 11 / 97 \\
08 / 11 / 97 \\
08 / 11 / 97 \\
08 / 11 / 97 \\
03 / 06 / 97 \\
03 / 06 / 97 \\
03 / 06 / 97 \\
03 / 06 / 97 \\
03 / 06 / 97 \\
08 / 12 / 97 \\
08 / 12 / 97 \\
08 / 12 / 97 \\
08 / 12 / 97 \\
08 / 12 / 97 \\
08 / 12 / 97 \\
08 / 12 / 97 \\
08 / 12 / 97 \\
08 / 12 / 97 \\
03 / 06 / 97 \\
08 / 12 / 97 \\
08 / 12 / 97 \\
08 / 12 / 97 \\
08 / 12 / 97 \\
03 / 06 / 97 \\
03 / 06 / 97 \\
03 / 06 / 97 \\
03 / 06 / 97 \\
03 / 06 / 97 \\
08 / 12 / 97 \\
08 / 12 / 97 \\
08 / 12 / 97 \\
08 / 12 / 97 \\
08 / 12 / 97 \\
08 / 12 / 97 \\
08 / 12 / 97 \\
08 / 12 / 97 \\
08 / 12 / 97 \\
03 / 06 / 97 \\
08 / 12 / 97 \\
08 / 12 / 97 \\
08 / 12 / 97 \\
08 / 12 / 97 \\
03 / 04 / 97 \\
03 / 04 / 97 \\
03 / 04 / 97 \\
03 / 04 / 97 \\
03 / 04 / 97 \\
03 / 04 / 97 \\
07 / 24 / 97 \\
07 / 24 / 97\end{array}$ & 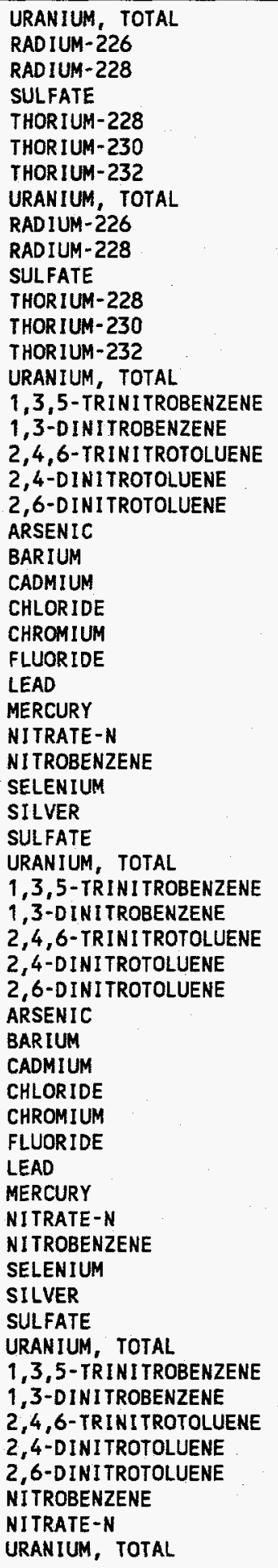 & $\begin{array}{l}1.48 \\
60.17 \\
1.60 \\
112 \\
0.102 \\
0.169 \\
0.035 \\
7.01 \\
0.567 \\
0.697 \\
74.2 \\
60.04 \\
0.232 \\
\text { CO.00 } \\
0.548 \\
\text { ND } \\
\text { ND } \\
\text { ND } \\
\text { ND } \\
\text { ND } \\
\text { ND } \\
201 \\
\text { ND } \\
26.4 \\
0.96 \\
0.799 \\
1.3 \\
\text { ND } \\
1.41 \\
\text { ND } \\
\text { ND } \\
2.2 \\
173 \\
8.46 \\
\text { ND } \\
\text { ND } \\
\text { ND } \\
\text { ND } \\
\text { ND } \\
\text { ND } \\
326 \\
\text { ND } \\
6.19 \\
0.77 \\
0.760 \\
\text { ND } \\
\text { ND } \\
0.370 \\
\text { ND } \\
\text { ND } \\
6.1 \\
48.3 \\
9.25 \\
\text { ND } \\
\text { ND } \\
\text { ND } \\
\text { ND } \\
\text { ND } \\
\text { ND } \\
68.4 \\
0.519\end{array}$ & $\begin{array}{l}0.0643 \\
0.264 \\
0.551 \\
0.900 \\
0.0578 \\
0.0342 \\
0.0182 \\
0.0643 \\
0.299 \\
0.668 \\
0.900 \\
0.0733 \\
0.0254 \\
0.0318 \\
0.0643 \\
0.030 \\
0.090 \\
0.030 \\
0.030 \\
0.010 \\
2.5 \\
0.2 \\
0.3 \\
1.28 \\
0.5 \\
0.0200 \\
1.2 \\
0.1 \\
0.00700 \\
0.030 \\
2.3 \\
0.6 \\
1.80 \\
0.0643 \\
0.030 \\
0.090 \\
0.030 \\
0.030 \\
0.010 \\
2.5 \\
0.2 \\
0.3 \\
0.064 \\
0.5 \\
0.020 \\
1.2 \\
0.1 \\
0.007 \\
0.030 \\
2.3 \\
0.6 \\
0.900 \\
0.0643 \\
0.030 \\
0.090 \\
0.030 \\
0.030 \\
0.010 \\
0.030 \\
0.28 \\
0.0643 \\
\end{array}$ & 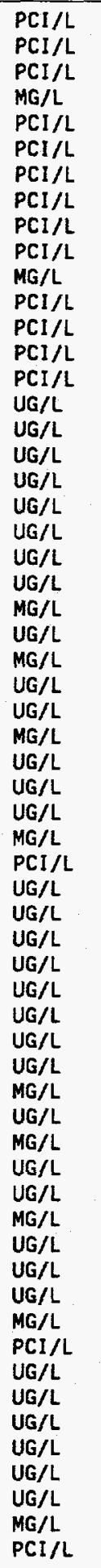 & $\begin{array}{l}\text { ASTM } 5174-91 \\
\text { EPA } 903.1 \\
\text { EPA } 904.0 \\
\text { EPA } 300.0 \\
\text { HASL } 300 \\
\text { HASL } 300 \\
\text { HASL } 300 \\
\text { ASTM } 5174-91 \\
\text { EPA } 903.1 \\
\text { EPA 904.0 } \\
\text { EPA } 300.0 \\
\text { HASL } 300 \\
\text { HASL } 300 \\
\text { HASL } 300 \\
\text { ASTM } 5174-91 \\
\text { USATHAMA } \\
\text { USATHAMA } \\
\text { USATHAMA } \\
\text { USATHAMA } \\
\text { USATHAMA } \\
\text { EPA CLP } \\
\text { EPA CLP } \\
\text { EPA CLP } \\
\text { EPA } 300.0 \\
\text { EPA CLP } \\
\text { EPA } 300.0 \\
\text { EPA CLP } \\
\text { EPA CLP } \\
\text { EPA } 353.1 \\
\text { USATHAMA } \\
\text { EPA CLP } \\
\text { EPA CLP } \\
\text { EPA } 300.0 \\
\text { ASTM } 5174-91 \\
\text { USATHAMA } \\
\text { USATHAMA } \\
\text { USATHAMA } \\
\text { USATHAMA } \\
\text { USATHAMA } \\
\text { EPA CLP } \\
\text { EPA CLP } \\
\text { EPA CLP } \\
\text { EPA } 300.0 \\
\text { EPA CLP } \\
\text { EPA } 300.0 \\
\text { EPA CLP } \\
\text { EPA CLP } \\
\text { EPA } 353.1 \\
\text { USATHAMA } \\
\text { EPA CLP } \\
\text { EPA CLP } \\
\text { EPA } 300.0 \\
\text { ASTM } 5174-91 \\
\text { USATHAMA } \\
\text { USATHAMA } \\
\text { USATHAMA } \\
\text { USATHAMA } \\
\text { USATHAMA } \\
\text { USATHAMA } \\
\text { EPA } 353.1 \\
\text { ASTM } 5174-91 \\
\text { AST }\end{array}$ \\
\hline
\end{tabular}




\begin{tabular}{|c|c|c|c|c|c|c|}
\hline WSSRAP_ID & DATE_SAM & PARAMETER & CONC & $\mathrm{DL}$ & UNITS & METHOD \\
\hline $\begin{array}{l}\text { GW-2013-0997 } \\
\text { GW-2013-1197 } \\
\text { GW-2013-0997 } \\
\text { GW-2013-1197 } \\
\text { GW-2013-0997 } \\
\text { GW-2013-1197 } \\
\text { GW-2013-0997 } \\
\text { GW-2013-1197 } \\
\text { GW-2013-0997 } \\
\text { GW-2013-1197 } \\
\text { GW-2013-0997 } \\
\text { GW-2013-1197 } \\
\text { GW-2013-0997 } \\
\text { GW-2013-1197 } \\
\text { GW-2013-0997 } \\
\text { GW-2013-1197 } \\
\text { GW-2013-0997 } \\
\text { GW-2013-1197 } \\
\text { GW-2013-0997 } \\
\text { GW-2013-1197 } \\
\text { GW-2013-0997 } \\
\text { GW-2013-1197 } \\
\text { GW-2013-0997 } \\
\text { GW-2013-1197 } \\
\text { GW-2013-0997 } \\
\text { GW-2013-1197 } \\
\text { GW-2013-0997 } \\
\text { GW-2013-0997 } \\
\text { GW-2013-1197 } \\
\text { GW-2013-0997 } \\
\text { GW-2013-1197 } \\
\text { GW-2013-0997 } \\
\text { GW-2013-1197 } \\
\text { GW-2013-0997 } \\
\text { GW-2013-1197 } \\
\text { GW-2013-0997 } \\
\text { GW-2013-1197 } \\
\text { GW-2013-0997 } \\
\text { GW-2013-1197 } \\
\text { GW-2013-0997 } \\
\text { GW-2013-1197 } \\
\text { GW-2013-0997 } \\
\text { GW-2013-1197 } \\
\text { GW-2013-0997 } \\
\text { GW-2013-1197 } \\
\text { GW-2013-0997 } \\
\text { GW-2013-1197 } \\
\text { GW-2013-0997 } \\
\text { GW-2013-1197 } \\
\text { GW-2013-0997 } \\
\text { GW-2013-1197 } \\
\text { GW-2013-0997 } \\
\text { GW-2013-1197 } \\
\text { GW-2013-0997 } \\
\text { GW-2013-1197 } \\
\text { GW-2013-0997 } \\
\text { GW-2013-1197 } \\
\text { GW-2013-0997 } \\
\text { GW-2013-1197 }\end{array}$ & $\begin{array}{l}09 / 11 / 97 \\
11 / 06 / 97 \\
09 / 11 / 97 \\
11 / 06 / 97 \\
09 / 11 / 97 \\
11 / 06 / 97 \\
09 / 11 / 97 \\
11 / 06 / 97 \\
09 / 11 / 97 \\
11 / 06 / 97 \\
09 / 11 / 97 \\
11 / 06 / 97 \\
09 / 11 / 97 \\
11 / 06 / 97 \\
09 / 11 / 97 \\
11 / 06 / 97 \\
09 / 11 / 97 \\
11 / 06 / 97 \\
09 / 11 / 97 \\
11 / 06 / 97 \\
09 / 11 / 97 \\
11 / 06 / 97 \\
09 / 11 / 97 \\
11 / 06 / 97 \\
09 / 11 / 97 \\
11 / 06 / 97 \\
09 / 11 / 97 \\
09 / 11 / 97 \\
11 / 06 / 97 \\
09 / 11 / 97 \\
11 / 06 / 97 \\
09 / 11 / 97 \\
11 / 06 / 97 \\
09 / 11 / 97 \\
11 / 06 / 97 \\
09 / 11 / 97 \\
11 / 06 / 97 \\
09 / 11 / 97 \\
11 / 06 / 97 \\
09 / 11 / 97 \\
11 / 06 / 97 \\
09 / 11 / 97 \\
11 / 06 / 97 \\
09 / 11 / 97 \\
11 / 06 / 97 \\
09 / 11 / 97 \\
11 / 06 / 97 \\
09 / 11 / 97 \\
11 / 06 / 97 \\
09 / 11 / 97 \\
11 / 06 / 97 \\
09 / 11 / 97 \\
11 / 06 / 97 \\
09 / 11 / 97 \\
11 / 06 / 97 \\
09 / 11 / 97 \\
11 / 06 / 97 \\
09 / 11 / 97 \\
11 / 06 / 97 \\
09 / 11 / 97 \\
11 / 06 / 97\end{array}$ & $\begin{array}{l}1,1,1-T R I C H L O R O E T H A N E \\
1,1,1-T R I C H L O R O E T H A N E \\
1,1,2,2-T E T R A C H L O R O E T H A N E \\
1,1,2,2-T E T R A C H L O R O E T H A N E \\
1,1,2-T R I C H L O R O E T H A N E \\
1,1,2-T R I C H L O R O E T H A N E \\
1,1-D I C H L O R O E T H A N E \\
1,1-D I C H L O R O E T H A N E \\
1,1-D I C H L O R O E T H E N E \\
1,1-D I C H L O R O E T H E N E \\
1,2-D I C H L O R O E T H A N E \\
1,2-D I C H L O R O E T H A N E \\
1,2-D I C H L O R O E T H E N E \text { (TOTAL) } \\
1,2-D I C H L O R O E T H E N E \text { (TOTAL) } \\
1,2-D I C H L O R O P R O P A N E \\
1,2-D I C H L O R O P R O P A N E \\
2-\text { BUTANONE } \\
\text { 2-BUTANONE } \\
\text { 2-HEXANONE } \\
2-H E X A N O N E \\
4-M E T H Y-2-P E N T A N O N E \\
\text { 4-METHYL-2-PENTANONE } \\
\text { ACETONE } \\
\text { ACETONE } \\
\text { BENZENE } \\
\text { BENZENE } \\
\text { BENZENE, 1,4-DIMETHYL-(TIC) } \\
\text { BROMODICHLOROMETHANE } \\
\text { BROMODICHLOROMETHANE } \\
\text { BROMOFORM } \\
\text { BROMOFORM } \\
\text { BROMOMETHANE } \\
\text { BROMOMETHANE } \\
\text { CARBON DISULFIDE } \\
\text { CARBON DISULFIDE } \\
\text { CARBON TETRACHLORIDE } \\
\text { CARBON TETRACHLORIDE } \\
\text { CHLOROBENZENE } \\
\text { CHLOROBENZENE } \\
\text { CHLOROETHANE } \\
\text { CHLOROETHANE } \\
\text { CHLOROFORM } \\
\text { CHLOROFORM } \\
\text { CHLOROMETHANE } \\
\text { CHLOROMETHANE } \\
\text { CIS-1,3-DICHLOROPROPENE } \\
\text { CIS-1,3-DICHLOROPROPENE } \\
\text { DIBROMOCHLOROMETHANE } \\
\text { DIBROMOCHLOROMETHANE } \\
\text { ETHYL BENZENE } \\
\text { ETHYL BENZENE } \\
\text { METHYLENE CHLORIDE } \\
\text { METHYLENE CHLORIDE } \\
\text { STYRENE } \\
\text { STYRENE } \\
\text { TETRACHLOROETHENE } \\
\text { TETRACHLOROETHENE } \\
\text { TOLUENE } \\
\text { TOLUENE } \\
\text { TRANS- } 1,3-D I C H L O R O P R O P E N E ~ \\
\text { TRANS- } 1,3-D I C H L O R O P R O P E N E \\
\end{array}$ & $\begin{array}{l}\text { ND } \\
\text { ND } \\
\text { ND } \\
\text { ND } \\
\text { ND } \\
\text { ND } \\
\text { ND } \\
\text { ND } \\
\text { ND } \\
\text { ND } \\
\text { ND } \\
\text { ND } \\
\text { (9) } \\
\text { C6 } \\
\text { ND } \\
\text { ND } \\
\text { ND } \\
\text { ND } \\
\text { ND } \\
\text { ND } \\
\text { ND } \\
\text { ND } \\
\text { ND } \\
\text { ND } \\
\text { ND } \\
\text { ND } \\
\text { ND } \\
\text { ND } \\
\text { ND } \\
\text { ND } \\
\text { ND } \\
\text { ND } \\
\text { ND } \\
\text { ND } \\
\text { ND } \\
\text { ND } \\
\text { ND } \\
\text { ND } \\
\text { ND } \\
\text { ND } \\
\text { ND } \\
\text { ND } \\
\text { ND } \\
\text { ND } \\
\text { ND } \\
\text { ND } \\
\text { ND } \\
\text { ND } \\
\text { ND } \\
\text { ND } \\
\text { ND } \\
\text { ND } \\
\text { ND } \\
\text { ND } \\
\text { ND } \\
\text { ND } \\
\text { ND } \\
\text { ND } \\
\text { ND } \\
\text { ND } \\
\text { ND }\end{array}$ & $\begin{array}{l}10 \\
10 \\
10 \\
10 \\
10 \\
10 \\
10 \\
10 \\
10 \\
10 \\
10 \\
10 \\
10 \\
10 \\
10 \\
10 \\
10 \\
10 \\
10 \\
10 \\
10 \\
10 \\
10 \\
10 \\
10 \\
10 \\
10 \\
10 \\
10 \\
10 \\
10\end{array}$ & $\begin{array}{l}U G / L \\
U G / L \\
U G / L \\
U G / L \\
U G / L \\
U G / L \\
U G / L \\
U G / L \\
U G / L \\
U G / L \\
U G / L \\
U G / L \\
U G / L \\
U G / L \\
U G / L \\
U G / L \\
U G / L \\
U G / L \\
U G / L \\
U G / L \\
U G / L \\
U G / L \\
U G / L \\
U G / L \\
U G / L \\
U G / L \\
U G / L \\
U G / L \\
U G / L \\
U G / L \\
U G / L \\
U G / L \\
U G / L \\
U G / L \\
U G / L \\
U G / L \\
U G / L \\
U G / L \\
U G / L \\
U G / L \\
U G / L \\
U G / L \\
U G / L \\
U G / L \\
U G / L \\
U G / L \\
U G / L \\
U G / L \\
U G / L \\
U G / L \\
U G / L \\
U G / L \\
U G / L \\
U G / L \\
U G / L \\
U G / L \\
U G / L \\
U G / L \\
U G / L \\
U G / L \\
U G / L\end{array}$ & 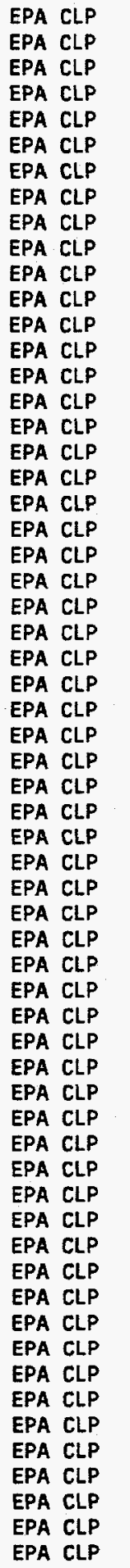 \\
\hline
\end{tabular}




\begin{tabular}{|c|c|c|c|c|c|c|}
\hline WSSRAP_ID & DATE_SAM & PARAMETER & CONC & DL & UNITS & METHOD \\
\hline 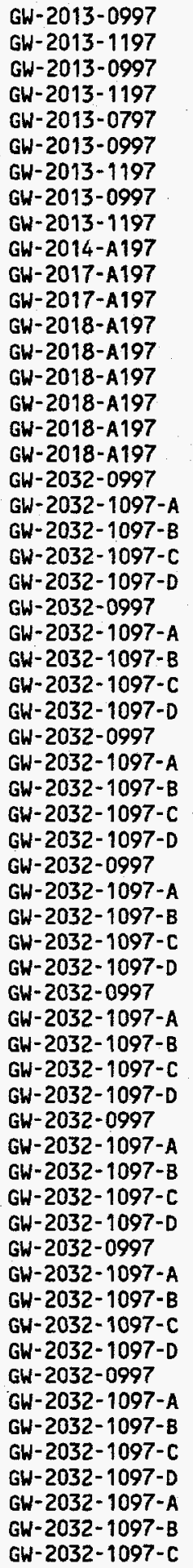 & $\begin{array}{l}09 / 11 / 97 \\
11 / 06 / 97 \\
09 / 11 / 97 \\
11 / 06 / 97 \\
07 / 24 / 97 \\
09 / 11 / 97 \\
11 / 06 / 97 \\
09 / 11 / 97 \\
11 / 06 / 97 \\
07 / 24 / 97 \\
07 / 24 / 97 \\
07 / 24 / 97 \\
08 / 20 / 97 \\
08 / 20 / 97 \\
08 / 20 / 97 \\
08 / 20 / 97 \\
08 / 20 / 97 \\
08 / 20 / 97 \\
09 / 11 / 97 \\
10 / 01 / 97 \\
10 / 02 / 97 \\
10 / 07 / 97 \\
10 / 08 / 97 \\
09 / 11 / 97 \\
10 / 01 / 97 \\
10 / 02 / 97 \\
10 / 07 / 97 \\
10 / 08 / 97 \\
09 / 11 / 97 \\
10 / 01 / 97 \\
10 / 02 / 97 \\
10 / 07 / 97 \\
10 / 08 / 97 \\
09 / 11 / 97 \\
10 / 01 / 97 \\
10 / 02 / 97 \\
10 / 07 / 97 \\
10 / 08 / 97 \\
09 / 11 / 97 \\
10 / 01 / 97 \\
10 / 02 / 97 \\
10 / 07 / 97 \\
10 / 08 / 97 \\
09 / 11 / 97 \\
10 / 01 / 97 \\
10 / 02 / 97 \\
10 / 07 / 97 \\
10 / 08 / 97 \\
09 / 11 / 97 \\
10 / 01 / 97 \\
10 / 02 / 97 \\
10 / 07 / 97 \\
10 / 08 / 97 \\
09 / 11 / 97 \\
10 / 01 / 97 \\
10 / 02 / 97 \\
10 / 07 / 97 \\
10 / 08 / 97 \\
10 / 01 / 97 \\
10 / 02 / 97 \\
10 / 07 / 97\end{array}$ & 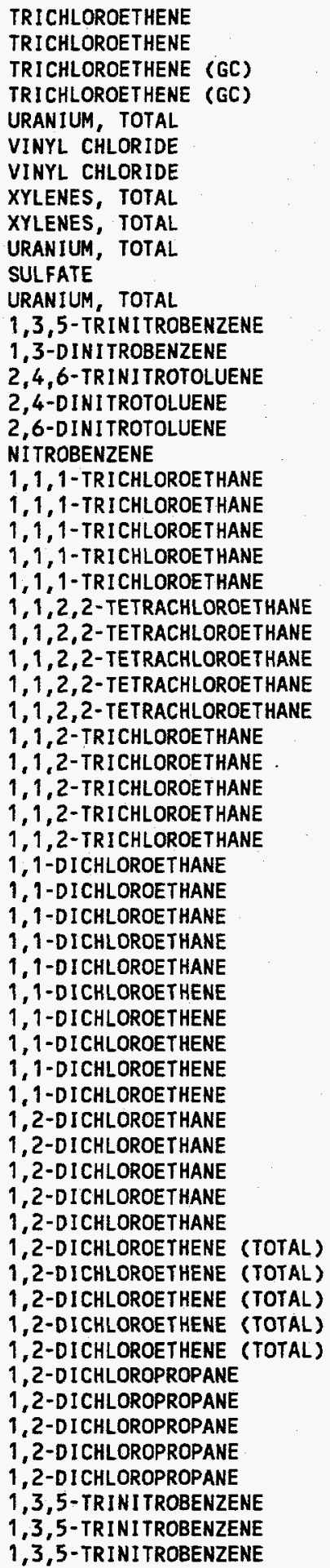 & 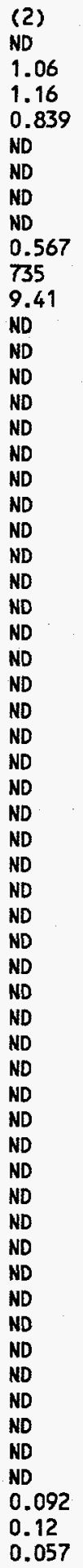 & 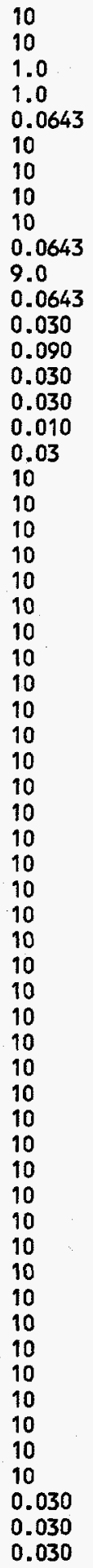 & 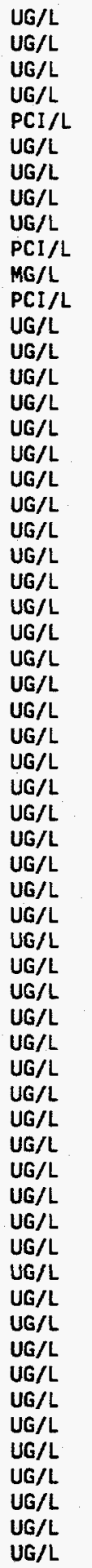 & $\begin{array}{l}\text { EPA CLP } \\
\text { EPA CLP } \\
\text { EPA 601 } \\
\text { EPA 601 } \\
\text { ASTM } 5174-91 \\
\text { EPA CLP } \\
\text { EPA CLP } \\
\text { EPA CLP } \\
\text { EPA CLP } \\
\text { ASTM } 5174-91 \\
\text { EPA } 300.0 \\
\text { ASTM } 5174-91 \\
\text { USATHAMA } \\
\text { USATHAMA } \\
\text { USATHAMA } \\
\text { USATHAMA } \\
\text { USATHAMA } \\
\text { USATHAMA } \\
\text { EPA CLP } \\
\text { EPA CLP } \\
\text { EPA CLP } \\
\text { EPA CLP } \\
\text { EPA CLP } \\
\text { EPA CLP } \\
\text { EPA CLP } \\
\text { EPA CLP } \\
\text { EPA CLP } \\
\text { EPA CLP } \\
\text { EPA CLP } \\
\text { EPA CLP } \\
\text { EPA CLP } \\
\text { EPA CLP } \\
\text { EPA CLP } \\
\text { EPA CLP } \\
\text { EPA CLP } \\
\text { EPA CLP } \\
\text { EPA CLP } \\
\text { EPA CLP } \\
\text { EPA CLP } \\
\text { EPA CLP } \\
\text { EPA CLP } \\
\text { EPA CLP } \\
\text { EPA CLP } \\
\text { EPA CLP } \\
\text { EPA CLP } \\
\text { EPA CLP } \\
\text { EPA CLP } \\
\text { EPA CLP } \\
\text { EPA CLP } \\
\text { EPA CLP } \\
\text { EPA CLP } \\
\text { EPA CLP } \\
\text { EPA CLP } \\
\text { EPA CLP } \\
\text { EPA CLP } \\
\text { EPA CLP } \\
\text { EPA CLP } \\
\text { EPA CLP } \\
\text { USATHAMA } \\
\text { USATHAMA } \\
\text { USATHAMA } \\
\end{array}$ \\
\hline
\end{tabular}




\begin{tabular}{|c|c|c|c|c|c|c|}
\hline WSSRAP_ID & DATE_SAM & PARAMETER & CONC & DL & UNITS & METHOD \\
\hline 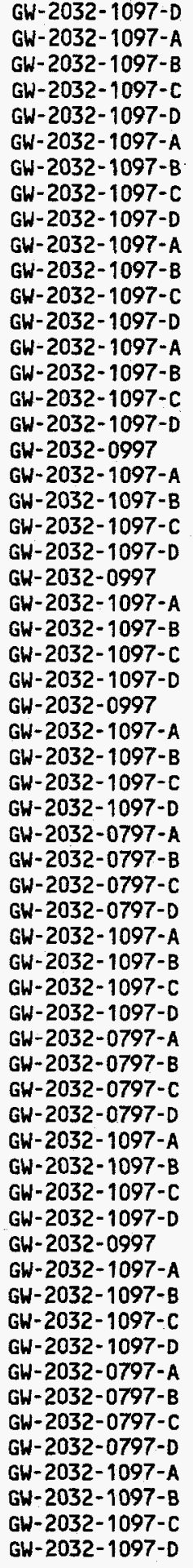 & $\begin{array}{l}10 / 08 / 97 \\
10 / 01 / 97 \\
10 / 02 / 97 \\
10 / 07 / 97 \\
10 / 08 / 97 \\
10 / 01 / 97 \\
10 / 02 / 97 \\
10 / 07 / 97 \\
10 / 08 / 97 \\
10 / 01 / 97 \\
10 / 02 / 97 \\
10 / 07 / 97 \\
10 / 08 / 97 \\
10 / 01 / 97 \\
10 / 02 / 97 \\
10 / 07 / 97 \\
10 / 08 / 97 \\
09 / 11 / 97 \\
10 / 01 / 97 \\
10 / 02 / 97 \\
10 / 07 / 97 \\
10 / 08 / 97 \\
09 / 11 / 97 \\
10 / 01 / 97 \\
10 / 02 / 97 \\
10 / 07 / 97 \\
10 / 08 / 97 \\
09 / 11 / 97 \\
10 / 01 / 97 \\
10 / 02 / 97 \\
10 / 07 / 97 \\
10 / 08 / 97 \\
07 / 01 / 97 \\
07 / 01 / 97 \\
07 / 02 / 97 \\
07 / 02 / 97 \\
10 / 01 / 97 \\
10 / 02 / 97 \\
10 / 07 / 97 \\
10 / 08 / 97 \\
07 / 01 / 97 \\
07 / 01 / 97 \\
07 / 02 / 97 \\
07 / 02 / 97 \\
10 / 01 / 97 \\
10 / 02 / 97 \\
10 / 07 / 97 \\
10 / 08 / 97 \\
09 / 11 / 97 \\
10 / 01 / 97 \\
10 / 02 / 97 \\
10 / 07 / 97 \\
10 / 08 / 97 \\
07 / 01 / 97 \\
07 / 01 / 97 \\
07 / 02 / 97 \\
07 / 02 / 97 \\
10 / 01 / 97 \\
10 / 02 / 97 \\
10 / 07 / 97 \\
10 / 08 / 97\end{array}$ & 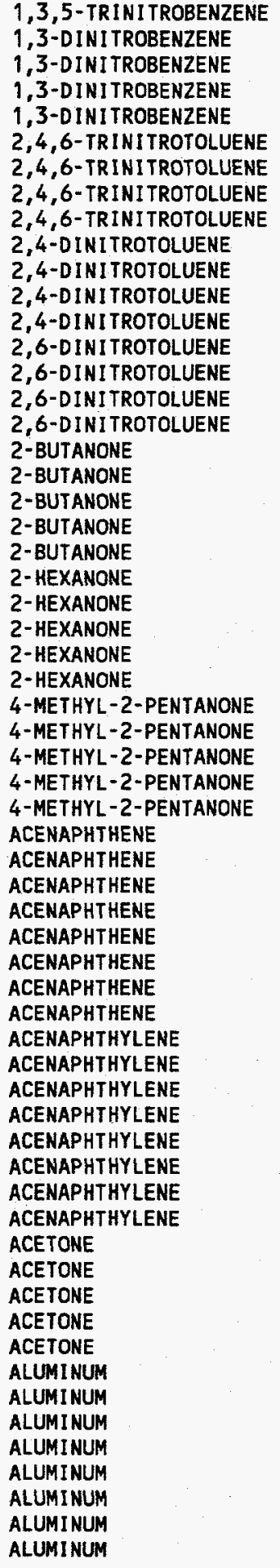 & $\begin{array}{l}0.16 \\
\text { ND } \\
\text { ND } \\
\text { ND } \\
0.22 \\
0.34 \\
0.17 \\
0.41 \\
0.033 \\
0.054 \\
\text { C.02 } \\
0.066 \\
0.12 \\
0.19 \\
0.10 \\
\text { O.24 } \\
\text { ND } \\
\text { ND } \\
\text { ND } \\
\text { ND } \\
\text { ND } \\
\text { ND } \\
\text { ND } \\
\text { ND } \\
\text { ND } \\
\text { ND } \\
\text { ND } \\
\text { ND } \\
\text { ND } \\
\text { ND } \\
\text { ND } \\
\text { ND } \\
\text { ND } \\
\text { ND } \\
\text { ND } \\
\text { ND } \\
\text { ND } \\
\text { ND } \\
\text { ND } \\
\text { ND } \\
\text { ND } \\
\text { ND } \\
\text { ND } \\
\text { ND } \\
\text { ND } \\
\text { ND } \\
\text { ND } \\
\text { ND } \\
\text { ND } \\
\text { ND } \\
\text { ND } \\
\text { ND } \\
\text { 35.8 } \\
110 \\
82.0 \\
107 \\
249 \\
\text { 392 } \\
171 \\
161 \\
\text { Din }\end{array}$ & $\begin{array}{l}0.030 \\
0.090 \\
0.090 \\
0.090 \\
0.090 \\
0.030 \\
0.030 \\
0.030 \\
0.030 \\
0.030 \\
0.030 \\
0.030 \\
0.030 \\
0.010 \\
0.010 \\
0.010 \\
0.010 \\
10 \\
10 \\
10 \\
10 \\
10 \\
10 \\
10 \\
10 \\
10 \\
10 \\
10 \\
10 \\
10 \\
10 \\
10 \\
1.00 \\
1.00 \\
1.00 \\
1.00 \\
0.5 \\
0.5 \\
0.5 \\
0.5 \\
1.00 \\
1.00 \\
1.00 \\
1.00 \\
0.5 \\
0.5 \\
0.5 \\
0.5 \\
10 \\
10 \\
10 \\
10 \\
10 \\
14.5 \\
14.5 \\
14.5 \\
14.5 \\
27 \\
27.0 \\
27.0 \\
27.0\end{array}$ & 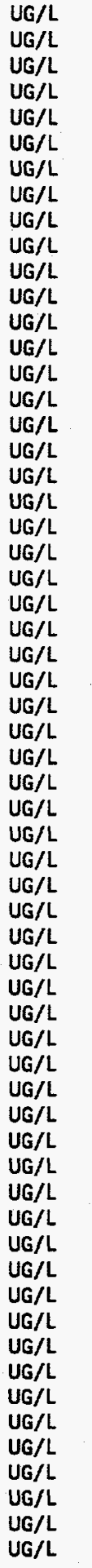 & $\begin{array}{l}\text { USATHAMA } \\
\text { USATHAMA } \\
\text { USATHAMA } \\
\text { USATHAMA } \\
\text { USATHAMA } \\
\text { USATHAMA } \\
\text { USATHAMA } \\
\text { USATHAMA } \\
\text { USATHAMA } \\
\text { USATHAMA } \\
\text { USATHAMA } \\
\text { USATHAMA } \\
\text { USATHAMA } \\
\text { USATHAMA } \\
\text { USATHAMA } \\
\text { USATHAMA } \\
\text { USATHAMA } \\
\text { EPA CLP } \\
\text { EPA CLP } \\
\text { EPA CLP } \\
\text { EPA CLP } \\
\text { EPA CLP } \\
\text { EPA CLP } \\
\text { EPA CLP } \\
\text { EPA CLP } \\
\text { EPA CLP } \\
\text { EPA CLP } \\
\text { EPA CLP } \\
\text { EPA CLP } \\
\text { EPA CLP } \\
\text { EPA CLP } \\
\text { EPA CLP } \\
\text { EPA } 8270 A \\
\text { EPA } 8270 A \\
\text { EPA } 8270 A \\
\text { EPA } 8270 A \\
\text { EPA } 8310 \\
\text { EPA } 8310 \\
\text { EPA } 8310 \\
\text { SH846 } 8310 \\
\text { EPA } 8270 A \\
\text { EPA } 8270 A \\
\text { EPA } 8270 A \\
\text { EPA } 8270 A \\
\text { EPA } 8310 \\
\text { EPA } 601010 A \\
\text { EPA } 8310 \\
\text { EPA } 8310 \\
\text { EPA } 6010 A \\
\text { EPA } 6010910 \\
\text { EPA CLP } 6010 A \\
\text { EPA } 6010 A \\
\text { EPA CLP } \\
\text { EPA CLP } \\
\text { EPA CLP } \\
\text { EPA } 6010 A \\
\text { EPA } \\
\text { EA }\end{array}$ \\
\hline
\end{tabular}




\begin{tabular}{|c|c|c|c|c|c|c|}
\hline WSSRAP_ID & DATE_SAM & PARAMETER & CONC & $D L$ & UNITS & METHOD \\
\hline $\begin{array}{l}G W-2032-0797-A \\
G W-2032-0797-B \\
G W-2032-0797-C \\
G W-2032-0797-D \\
G W-2032-1097-A \\
G W-2032-1097-B \\
G W-2032-1097-C \\
G W-2032-1097-D \\
G W-2032-0797-A \\
G W-2032-0797-B \\
G W-2032-0797-C \\
G W-2032-0797-D \\
G W-2032-1097-A \\
G W-2032-1097-B \\
G W-2032-1097-C \\
G W-2032-1097-D \\
G W-2032-0797-A \\
G W-2032-0797-B \\
G W-2032-0797-C \\
G W-2032-0797-D \\
G W-2032-1097-A \\
G W-2032-1097-B \\
G W-2032-1097-C \\
G W-2032-1097-D \\
G W-2032-0797-A \\
G W-2032-0797-B \\
G W-2032-0797-C \\
G W-2032-0797-D \\
G W-2032-1097-A \\
G W-2032-1097-B \\
G W-2032-1097-C \\
G W-2032-1097-D \\
G W-2032-0797-A \\
G W-2032-0797-B \\
G W-2032-0797-C \\
G W-2032-0797-D \\
G W-2032-1097-A \\
G W-2032-1097-B \\
G W-2032-1097-C \\
G W-2032-1097-D \\
G W-2032-0797-A \\
G W-2032-0797-B \\
G W-2032-0797-C \\
G W-2032-0797-D \\
G W-2032-1097-A \\
G W-2032-1097-B \\
G W-2032-1097-C \\
G W-2032-1097-D \\
G W-2032-0797-A \\
G W-2032-0797-B \\
G W-2032-0797-C \\
G W-2032-0797-D \\
G W-2032-1097-A \\
G W-2032-1097-B \\
G W-2032-1097-C \\
G W-2032-1097-D \\
G W-2032-0797-A \\
G W-2032-0797-B \\
G W-2032-0797-C \\
G W-2032-0797-D \\
G W-2032-1097-A\end{array}$ & $\begin{array}{l}07 / 01 / 97 \\
07 / 01 / 97 \\
07 / 02 / 97 \\
07 / 02 / 97 \\
10 / 01 / 97 \\
10 / 02 / 97 \\
10 / 07 / 97 \\
10 / 08 / 97 \\
07 / 01 / 97 \\
07 / 01 / 97 \\
07 / 02 / 97 \\
07 / 02 / 97 \\
10 / 01 / 97 \\
10 / 02 / 97 \\
10 / 07 / 97 \\
10 / 08 / 97 \\
07 / 01 / 97 \\
07 / 01 / 97 \\
07 / 02 / 97 \\
07 / 02 / 97 \\
10 / 01 / 97 \\
10 / 02 / 97 \\
10 / 07 / 97 \\
10 / 08 / 97 \\
07 / 01 / 97 \\
07 / 01 / 97 \\
07 / 02 / 97 \\
07 / 02 / 97 \\
10 / 01 / 97 \\
10 / 02 / 97 \\
10 / 07 / 97 \\
10 / 08 / 97 \\
07 / 01 / 97 \\
07 / 01 / 97 \\
07 / 02 / 97 \\
07 / 02 / 97 \\
10 / 01 / 97 \\
10 / 02 / 97 \\
10 / 07 / 97 \\
10 / 08 / 97 \\
07 / 01 / 97 \\
07 / 01 / 97 \\
07 / 02 / 97 \\
07 / 02 / 97 \\
10 / 01 / 97 \\
10 / 02 / 97 \\
10 / 07 / 97 \\
10 / 08 / 97 \\
07 / 01 / 97 \\
07 / 01 / 97 \\
07 / 02 / 97 \\
07 / 02 / 97 \\
10 / 01 / 97 \\
10 / 02 / 97 \\
10 / 07 / 97 \\
10 / 08 / 97 \\
07 / 01 / 97 \\
07 / 01 / 97 \\
07 / 02 / 97 \\
07 / 02 / 97 \\
10 / 01 / 97\end{array}$ & 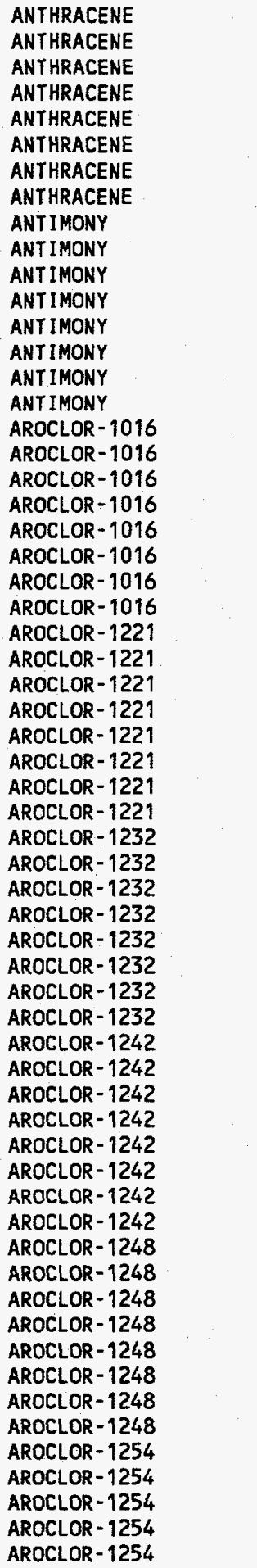 & $\begin{array}{l}\text { ND } \\
\text { ND } \\
\text { ND } \\
\text { ND } \\
\text { ND } \\
\text { ND } \\
\text { ND } \\
1.9 \\
\text { ND } \\
\text { ND } \\
\text { ND } \\
\text { ND } \\
\text { ND } \\
\text { ND } \\
\text { ND } \\
\text { ND } \\
\text { ND } \\
\text { ND } \\
\text { ND } \\
\text { ND } \\
\text { ND } \\
\text { ND } \\
\text { ND } \\
\text { ND } \\
\text { ND } \\
\text { ND } \\
\text { ND } \\
\text { ND } \\
\text { ND } \\
\text { ND } \\
\text { ND } \\
\text { ND } \\
\text { ND } \\
\text { ND } \\
\text { ND } \\
\text { ND } \\
\text { ND } \\
\text { ND } \\
\text { ND } \\
\text { ND } \\
\text { ND } \\
\text { ND } \\
\text { ND } \\
\text { ND } \\
\text { ND } \\
\text { ND } \\
\text { ND } \\
\text { ND } \\
\text { ND } \\
\text { ND } \\
\text { ND } \\
\text { ND } \\
\text { ND } \\
\text { ND } \\
\text { ND }\end{array}$ & $\begin{array}{l}1.00 \\
1.00 \\
1.00 \\
1.00 \\
0.5 \\
0.5 \\
0.5 \\
0.5 \\
1.3 \\
1.3 \\
1.3 \\
1.3 \\
11 \\
11.0 \\
11.0 \\
11.0 \\
0.10 \\
0.12 \\
0.10 \\
0.10 \\
1.0 \\
1.0 \\
1.0 \\
1.0 \\
0.20 \\
0.24 \\
0.20 \\
0.20 \\
1.0 \\
1.0 \\
1.0 \\
1.0 \\
0.10 \\
0.12 \\
0.10 \\
0.10 \\
1.0 \\
1.0 \\
1.0 \\
1.0 \\
0.10 \\
0.12 \\
0.10 \\
0.10 \\
1.0 \\
1.0 \\
1.0 \\
1.0 \\
0.10 \\
0.12 \\
0.10 \\
0.10 \\
1.0 \\
1.0 \\
1.0 \\
1.0 \\
0.10 \\
0.12 \\
0.10 \\
0.10 \\
1.0\end{array}$ & $\begin{array}{l}\text { UG/L } \\
U G / L \\
U G / L \\
U G / L \\
U G / L \\
U G / L \\
U G / L \\
U G / L \\
U G / L \\
U G / L \\
U G / L \\
U G / L \\
U G / L \\
U G / L \\
U G / L \\
U G / L \\
U G / L \\
U G / L \\
U G / L \\
U G / L \\
U G / L \\
U G / L \\
U G / L \\
U G / L \\
U G / L \\
U G / L \\
U G / L \\
U G / L \\
U G / L \\
U G / L \\
U G / L \\
U G / L \\
U G / L \\
U G / L \\
U G / L \\
U G / L \\
U G / L \\
U G / L \\
U G / L \\
U G / L \\
U G / L \\
U G / L \\
U G / L \\
U G / L \\
U G / L \\
U G / L \\
U G / L \\
U G / L \\
U G / L \\
U G / L \\
U G / L \\
U G / L \\
U G / L \\
U G / L \\
U G / L \\
U G / L \\
U G / L \\
U G / L \\
U G \\
U,\end{array}$ & 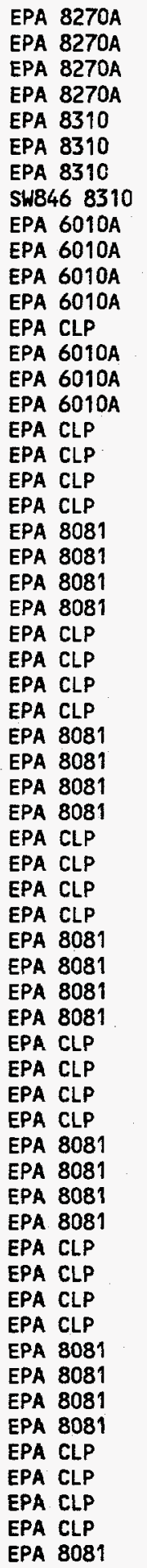 \\
\hline
\end{tabular}




\begin{tabular}{|c|c|c|c|c|c|c|}
\hline WSSRAP_ID & DATE_SAM & PARAMETER & CONC & $D L$ & UNITS & METHOD \\
\hline $\begin{array}{l}\text { GW-2032-1097-B } \\
\text { GW-2032-1097-C } \\
\text { GW-2032-1097-D } \\
\text { GW-2032-0797-A } \\
\text { GW-2032-0797-B } \\
\text { GW-2032-0797-C } \\
\text { GW-2032-0797-D } \\
\text { GW-2032-1097-A } \\
\text { GW-2032-1097-B } \\
G W-2032-1097-C \\
G W-2032-1097-D \\
G W-2032-0797-A \\
G W-2032-0797-B \\
G W-2032-0797-C \\
G W-2032-0797-D \\
G W-2032-1097-A \\
G W-2032-1097-B \\
G W-2032-1097-C \\
G W-2032-1097-D \\
G W-2032-0797-A \\
G W-2032-0797-B \\
G W-2032-0797-C \\
G W-2032-0797-D \\
G W-2032-1097-A \\
G W-2032-1097-B \\
G W-2032-1097-C \\
G W-2032-1097-D \\
G W-2032-0997 \\
G W-2032-1097-A \\
G W-2032-1097-B \\
G W-2032-1097-C \\
G W-2032-1097-D \\
G W-2032-1097-A \\
G W-2032-1097-A \\
G W-2032-0797-A \\
G W-2032-0797-B \\
G W-2032-0797-C \\
G W-2032-0797-D \\
G W-2032-1097-A \\
G W-2032-1097-B \\
G W-2032-1097-C \\
G W-2032-1097-D \\
G W-2032-0797-A \\
G W-2032-0797-B \\
G W-2032-0797-C \\
G W-2032-0797-D \\
G W-2032-1097-A \\
G W-2032-1097-B \\
G W-2032-1097-C \\
G W-2032-1097-D \\
G W-2032-0797-A \\
G W-2032-0797-B \\
G W-2032-0797-C \\
G W-2032-0797-D \\
G W-2032-1097-A \\
G W-2032-1097-B \\
G W-2032-1097-C \\
G W-2032-1097-D \\
G W-2032-0797-A \\
G W-2032-0797-B \\
G W-2032-0797-C\end{array}$ & $\begin{array}{l}10 / 02 / 97 \\
10 / 07 / 97 \\
10 / 08 / 97 \\
07 / 01 / 97 \\
07 / 01 / 97 \\
07 / 02 / 97 \\
07 / 02 / 97 \\
10 / 01 / 97 \\
10 / 02 / 97 \\
10 / 07 / 97 \\
10 / 08 / 97 \\
07 / 01 / 97 \\
07 / 01 / 97 \\
07 / 02 / 97 \\
07 / 02 / 97 \\
10 / 01 / 97 \\
10 / 02 / 97 \\
10 / 07 / 97 \\
10 / 08 / 97 \\
07 / 01 / 97 \\
07 / 01 / 97 \\
07 / 02 / 97 \\
07 / 02 / 97 \\
10 / 01 / 97 \\
10 / 02 / 97 \\
10 / 07 / 97 \\
10 / 08 / 97 \\
09 / 11 / 97 \\
10 / 01 / 97 \\
10 / 02 / 97 \\
10 / 07 / 97 \\
10 / 08 / 97 \\
10 / 01 / 97 \\
10 / 01 / 97 \\
07 / 01 / 97 \\
07 / 01 / 97 \\
07 / 02 / 97 \\
07 / 02 / 97 \\
10 / 01 / 97 \\
10 / 02 / 97 \\
10 / 07 / 97 \\
10 / 08 / 97 \\
07 / 01 / 97 \\
07 / 01 / 97 \\
07 / 02 / 97 \\
07 / 02 / 97 \\
10 / 01 / 97 \\
10 / 02 / 97 \\
10 / 07 / 97 \\
10 / 08 / 97 \\
07 / 01 / 97 \\
07 / 01 / 97 \\
07 / 02 / 97 \\
07 / 02 / 97 \\
10 / 01 / 97 \\
10 / 02 / 97 \\
10 / 07 / 97 \\
10 / 08 / 97 \\
07 / 01 / 97 \\
07 / 01 / 97 \\
07 / 02 / 97\end{array}$ & 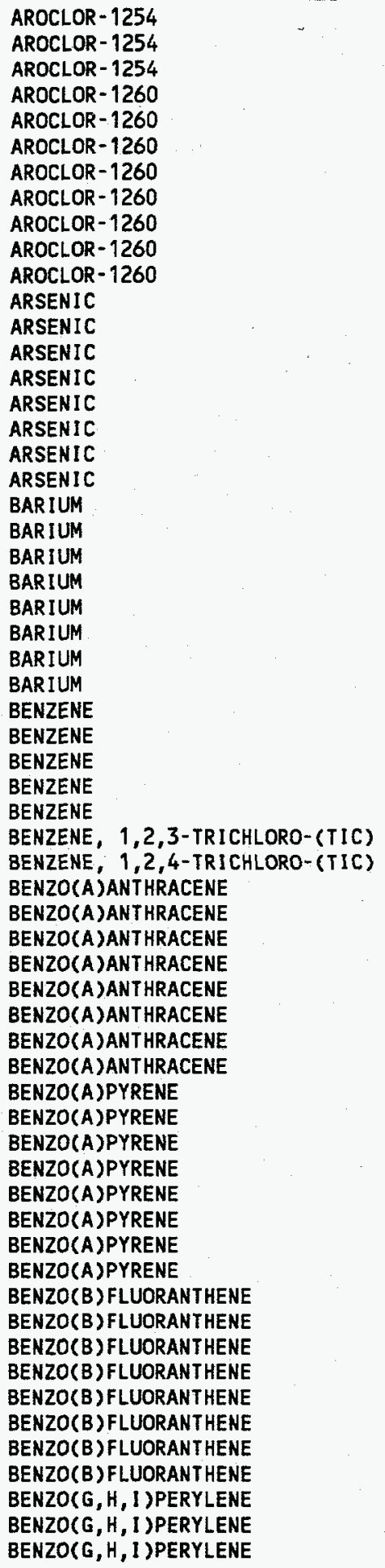 & $\begin{array}{l}\text { ND } \\
\text { ND } \\
\text { ND } \\
\text { ND } \\
\text { ND } \\
\text { ND } \\
\text { ND } \\
\text { ND } \\
\text { ND } \\
\text { ND } \\
\text { ND } \\
\text { ND } \\
\text { ND } \\
\text { ND } \\
\text { ND } \\
\text { ND } \\
\text { ND } \\
\text { ND } \\
\text { ND } \\
272 \\
304 \\
302 \\
288 \\
191 \\
215 \\
189 \\
212 \\
\text { ND } \\
\text { ND } \\
\text { ND } \\
\text { ND } \\
\text { ND } \\
6 \\
7 \\
\text { ND } \\
\text { ND } \\
\text { ND } \\
\text { ND } \\
\text { ND } \\
\text { ND } \\
\text { ND } \\
\text { ND } \\
\text { ND } \\
\text { ND } \\
\text { ND } \\
\text { ND } \\
\text { ND } \\
\text { ND } \\
\text { ND } \\
\text { ND } \\
\text { ND } \\
\text { ND } \\
\text { ND } \\
\text { ND } \\
\text { ND } \\
\text { ND } \\
\text { ND }\end{array}$ & $\begin{array}{l}1.0 \\
1.0 \\
1.0 \\
0.10 \\
0.12 \\
0.10 \\
0.10 \\
1.0 \\
1.0 \\
1.0 \\
1.0 \\
1.7 \\
1.7 \\
1.7 \\
1.7 \\
4.0 \\
4.0 \\
4.0 \\
4.0 \\
0.3 \\
0.3 \\
0.3 \\
0.3 \\
2.0 \\
2.0 \\
2.0 \\
2.0 \\
10 \\
10 \\
10 \\
10 \\
10 \\
0.00 \\
0.00 \\
1.00 \\
1.00 \\
1.00 \\
1.00 \\
0.5 \\
0.5 \\
0.5 \\
0.5 \\
1.00 \\
1.00 \\
1.00 \\
1.00 \\
0.5 \\
0.5 \\
0.5 \\
0.5 \\
1.00 \\
1.00 \\
1.00 \\
1.00 \\
0.5 \\
0.5 \\
0.5 \\
0.5 \\
1.00 \\
1.00 \\
1.00\end{array}$ & 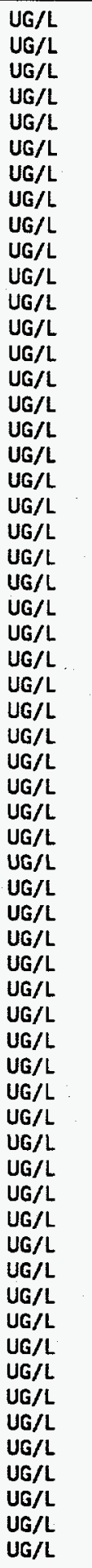 & $\begin{array}{l}\text { EPA } 8081 \\
\text { EPA } 8081 \\
\text { EPA } 8081 \\
\text { EPA CLP } \\
\text { EPA CLP } \\
\text { EPA CLP } \\
\text { EPA CLP } \\
\text { EPA } 8081 \\
\text { EPA } 8081 \\
\text { EPA } 8081 \\
\text { EPA } 8081 \\
\text { EPA } 6010 A \\
\text { EPA } 6010 A \\
\text { EPA } 6010 A \\
\text { EPA } 6010 A \\
\text { EPA CLP } \\
\text { EPA } 6010 A \\
\text { EPA } 6010 A \\
\text { EPA } 6010 A \\
\text { EPA } 6010 A \\
\text { EPA } 6010 A \\
\text { EPA } 6010 A \\
\text { EPA } 6010 A \\
\text { EPA CLP } \\
\text { EPA } 6010 A \\
\text { EPA } 6010 A \\
\text { EPA } 6010 A \\
\text { EPA CLP } \\
\text { EPA CLP } \\
\text { EPA CLP } \\
\text { EPA CLP } \\
\text { EPA CLP } \\
\text { EPA CLP } \\
\text { EPA CLP } \\
\text { EPA } 8270 A \\
\text { EPA } 8270 A \\
\text { EPA } 8270 A \\
\text { EPA } 8270 A \\
\text { EPA } 8310 \\
\text { EPA } 8310 \\
\text { EPA } 8310 \\
\text { SW846 } 8310 \\
\text { EPA } 8270 A \\
\text { EPA } 8270 A \\
\text { EPA } 8270 A \\
\text { EPA } 8270 A \\
\text { EPA } 8310 \\
\text { EPA } 8310 \\
\text { EPA } 8310 \\
\text { SW846 } 8310 \\
\text { EPA } 8270 A \\
\text { EPA } 8270 A \\
\text { EPA } 8270 A \\
\text { EPA } 8270 A \\
\text { EPA } 8310 \\
\text { EPA } 8310 \\
\text { EPA } 8310 \\
\text { SW846 } 8310 \\
\text { EPA } 8270 A \\
\text { EPA } 8270 A \\
\text { EPA } 8270 A\end{array}$ \\
\hline
\end{tabular}




\begin{tabular}{|c|c|c|c|c|c|c|}
\hline WSSRAP_ID & DATE_SAM & PARAMETER & CONC & $D L$ & UNITS & METHOD \\
\hline 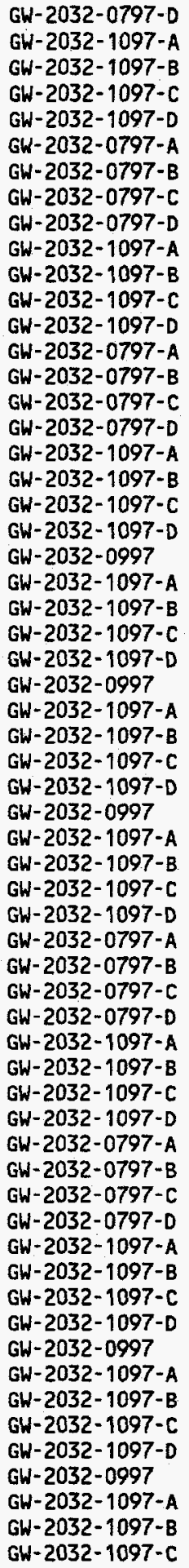 & $\begin{array}{l}07 / 02 / 97 \\
10 / 01 / 97 \\
10 / 02 / 97 \\
10 / 07 / 97 \\
10 / 08 / 97 \\
07 / 01 / 97 \\
07 / 01 / 97 \\
07 / 02 / 97 \\
07 / 02 / 97 \\
10 / 01 / 97 \\
10 / 02 / 97 \\
10 / 07 / 97 \\
10 / 08 / 97 \\
07 / 01 / 97 \\
07 / 01 / 97 \\
07 / 02 / 97 \\
07 / 02 / 97 \\
10 / 01 / 97 \\
10 / 02 / 97 \\
10 / 07 / 97 \\
10 / 08 / 97 \\
09 / 11 / 97 \\
10 / 01 / 97 \\
10 / 02 / 97 \\
10 / 07 / 97 \\
10 / 08 / 97 \\
09 / 11 / 97 \\
10 / 01 / 97 \\
10 / 02 / 97 \\
10 / 07 / 97 \\
10 / 08 / 97 \\
09 / 11 / 97 \\
10 / 01 / 97 \\
10 / 02 / 97 \\
10 / 07 / 97 \\
10 / 08 / 97 \\
07 / 01 / 97 \\
07 / 01 / 97 \\
07 / 02 / 97 \\
07 / 02 / 97 \\
10 / 01 / 97 \\
10 / 02 / 97 \\
10 / 07 / 97 \\
10 / 08 / 97 \\
07 / 01 / 97 \\
07 / 01 / 97 \\
07 / 02 / 97 \\
07 / 02 / 97 \\
10 / 01 / 97 \\
10 / 02 / 97 \\
10 / 07 / 97 \\
10 / 08 / 97 \\
09 / 11 / 97 \\
10 / 01 / 97 \\
10 / 02 / 97 \\
10 / 07 / 97 \\
10 / 08 / 97 \\
09 / 11 / 97 \\
10 / 01 / 97 \\
10 / 02 / 97 \\
10 / 07 / 97\end{array}$ & $\begin{array}{l}\text { BENZO(G, H, I)PERYLENE } \\
\text { BENZO(G,H, I)PERYLENE } \\
\text { BENZO(G,H,I)PERYLEN } \\
\text { BENZO(G,H, I)PERYLEN } \\
\text { BENZO(G,H,I)PERYLENE } \\
\text { BENZO(K)FLUORANTHENE } \\
\text { BENZO(K)FLUORANTHENE } \\
\text { BENZO(K)FLUORANTHENE } \\
\text { BENZO(K) FLUORANTHENE } \\
\text { BENZO(K)FLUORANTHENE } \\
\text { BENZO(K)FLUORANTHENE } \\
\text { BENZO(K)FLUORANTHENE } \\
\text { BENZO(K)FLUORANTHENE } \\
\text { BERYLLIUM } \\
\text { BERYLLIUM } \\
\text { BERYLLIUM } \\
\text { BERYLLIUM } \\
\text { BERYLLIUM } \\
\text { BERYLLIUM } \\
\text { BERYLLIUM } \\
\text { BERYLLIUM } \\
\text { BROMODICHLOROMETHANE } \\
\text { BROMODICHLOROMETHANE } \\
\text { BROMODICHLOROMETHANE } \\
\text { BROMODICHLOROMETHANE } \\
\text { BROMODICHLOROMETHANE } \\
\text { BROMOFORM } \\
\text { BROMOFORM } \\
\text { BROMOFORM } \\
\text { BROMOFORM } \\
\text { BROMOFORM } \\
\text { BROMOMETHANE } \\
\text { BROMOMETHANE } \\
\text { BROMOMETHANE } \\
\text { BROMOMETHANE } \\
\text { BROMOMETHANE } \\
\text { CADMIUM } \\
\text { CADMIUM } \\
\text { CADMIUM } \\
\text { CADMIUM } \\
\text { CADMIUM } \\
\text { CADMIUM } \\
\text { CADMIUM } \\
\text { CADMIUM } \\
\text { CALCIIUM } \\
\text { CALCIUM } \\
\text { CALCIUM } \\
\text { CALCIUM } \\
\text { CALCIUM } \\
\text { CALCIUM } \\
\text { CALCIUM } \\
\text { CALCIUM } \\
\text { CARBON DI SULFIDE } \\
\text { CARBON DI ISULFIDE } \\
\text { CARBON DI SULFIDE } \\
\text { CARBON DI SULFIDE } \\
\text { CARBON DI SULFIDE } \\
\text { CARBON TETRACHLORIDE } \\
\text { CARBON TETRACHLORIDE } \\
\text { CARBON TETRACHLORIDE } \\
\text { CARBON TETRACHLORIDE }\end{array}$ & 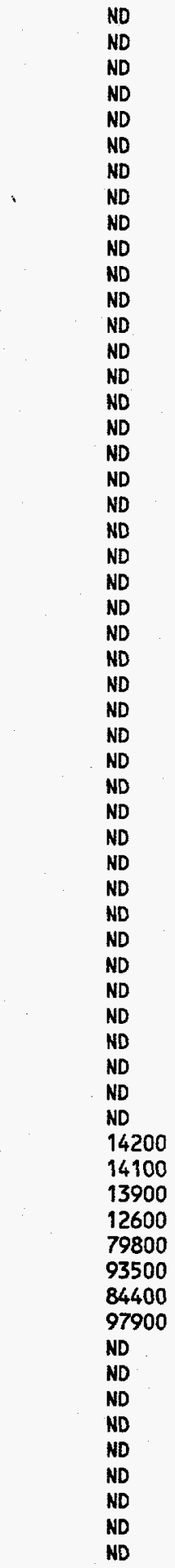 & $\begin{array}{l}1.00 \\
0.5 \\
0.5 \\
0.5 \\
0.5 \\
1.00 \\
1.00 \\
1.00 \\
1.00 \\
0.5 \\
0.5 \\
0.5 \\
0.5 \\
0.2 \\
0.2 \\
0.2 \\
0.2 \\
1.0 \\
1.0 \\
1.0 \\
1.0 \\
10 \\
10 \\
10 \\
10 \\
10 \\
10 \\
10 \\
10 \\
10 \\
10 \\
10 \\
10 \\
10 \\
10 \\
10 \\
0.3 \\
0.3 \\
0.3 \\
0.3 \\
2.0 \\
2.0 \\
2.0 \\
2.0 \\
3.0 \\
3.0 \\
3.0 \\
3.0 \\
10 \\
10 \\
10 \\
10 \\
13.0 \\
13.0 \\
10 \\
10 \\
10 \\
10\end{array}$ & 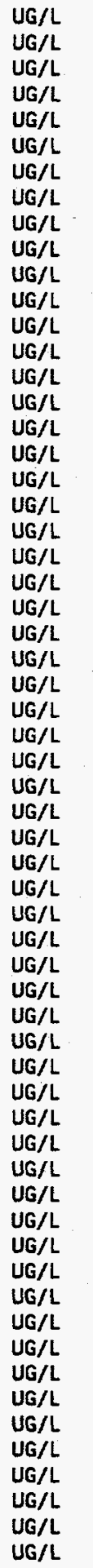 & 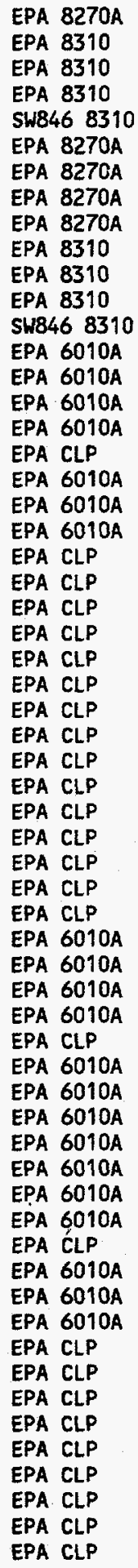 \\
\hline
\end{tabular}




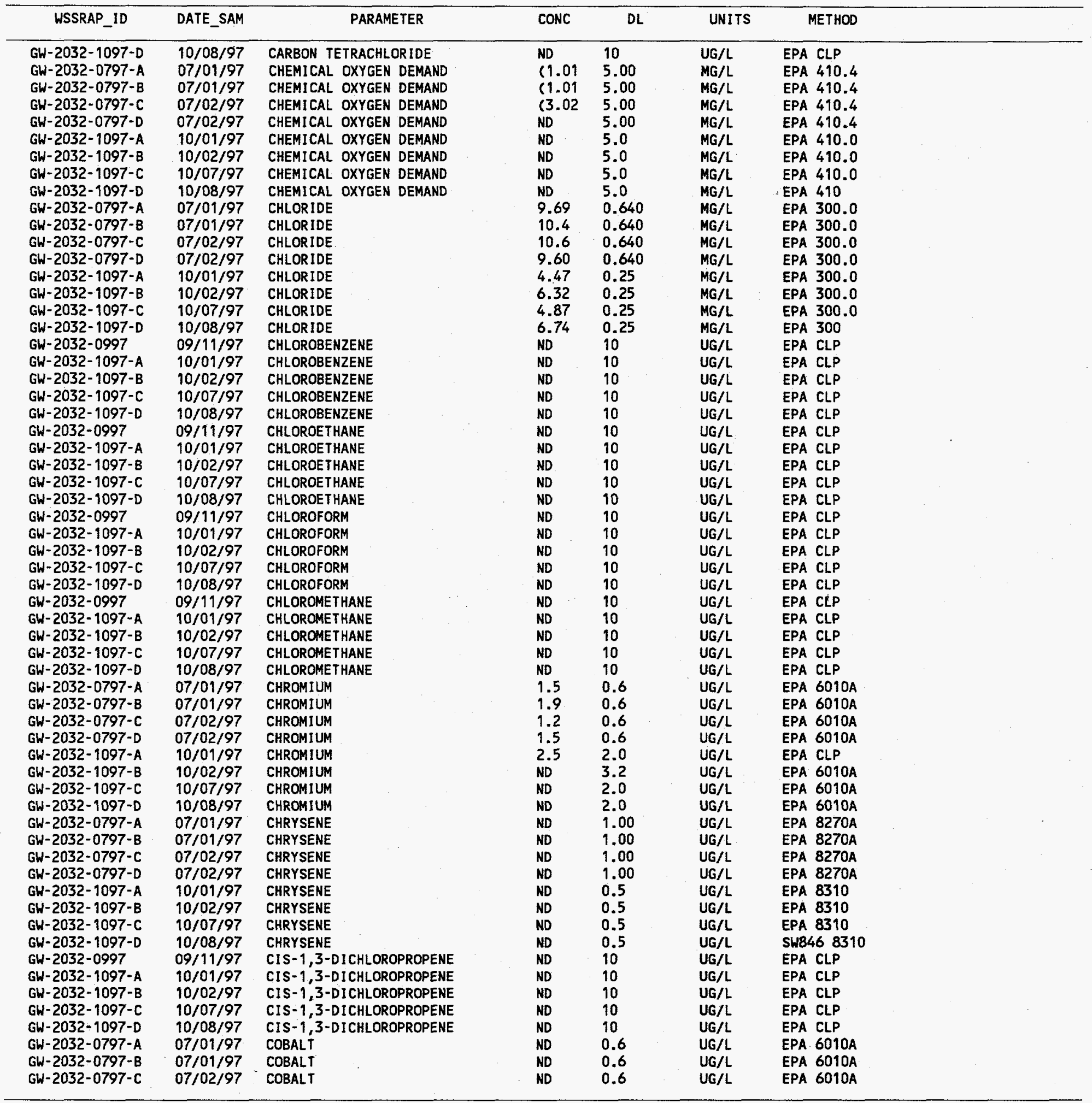




\begin{tabular}{|c|c|c|c|c|c|c|}
\hline WSSRAP_ID & DATE_SAM & PARAMETER & CONC & $\mathrm{DL}$ & UNITS & METHOD \\
\hline $\begin{array}{l}G W-2032-0797-D \\
G W-2032-1097-A \\
G W-2032-1097-B \\
G W-2032-1097-C \\
G W-2032-1097-D \\
G W-2032-0797-A \\
G W-2032-0797-B \\
G W-2032-0797-C \\
G W-2032-0797-D \\
G W-2032-1097-A \\
G W-2032-1097-B \\
G W-2032-1097-C \\
G W-2032-1097-D \\
G W-2032-0797-A \\
G W-2032-0797-B \\
G W-2032-0797-C \\
G W-2032-0797-D \\
G W-2032-1097-A \\
G W-2032-1097-B \\
G W-2032-1097-C \\
G W-2032-1097-D \\
G W-2032-1097-D \\
G W-2032-0797-A \\
G W-2032-0797-B \\
G W-2032-0797-C \\
G W-2032-0797-D \\
G W-2032-1097-A \\
G W-2032-1097-B \\
G W-2032-1097-C \\
G W-2032-0997 \\
G W-2032-1097-A \\
G W-2032-1097-B \\
G W-2032-1097-C \\
G W-2032-1097-D \\
G W-2032-0997 \\
G W-2032-1097-A \\
G W-2032-1097-B \\
G W-2032-1097-C \\
G W-2032-1097-D \\
G W-2032-0797-A \\
G W-2032-0797-B \\
G W-2032-0797-C \\
G W-2032-0797-D \\
G W-2032-1097-A \\
G W-2032-1097-B \\
G W-2032-1097-C \\
G W-2032-1097-D \\
G W-2032-0797-A \\
G W-2032-0797-B \\
G W-2032-0797-C \\
G W-2032-0797-D \\
G W-2032-1097-A \\
G W-2032-1097-B \\
G W-2032-1097-C \\
G W-2032-1097-D \\
G W-2032-0797-A \\
G W-2032-0797-B \\
G W-2032-0797-C \\
G W-2032-0797-D \\
G W-2032-1097-A \\
G W-2032-1097-B\end{array}$ & $\begin{array}{l}07 / 02 / 97 \\
10 / 01 / 97 \\
10 / 02 / 97 \\
10 / 07 / 97 \\
10 / 08 / 97 \\
07 / 01 / 97 \\
07 / 01 / 97 \\
07 / 02 / 97 \\
07 / 02 / 97 \\
10 / 01 / 97 \\
10 / 02 / 97 \\
10 / 07 / 97 \\
10 / 08 / 97 \\
07 / 01 / 97 \\
07 / 01 / 97 \\
07 / 02 / 97 \\
07 / 02 / 97 \\
10 / 01 / 97 \\
10 / 02 / 97 \\
10 / 07 / 97 \\
10 / 08 / 97 \\
10 / 08 / 97 \\
07 / 01 / 97 \\
07 / 01 / 97 \\
07 / 02 / 97 \\
07 / 02 / 97 \\
10 / 01 / 97 \\
10 / 02 / 97 \\
10 / 07 / 97 \\
09 / 11 / 97 \\
10 / 01 / 97 \\
10 / 02 / 97 \\
10 / 07 / 97 \\
10 / 08 / 97 \\
09 / 11 / 97 \\
10 / 01 / 97 \\
10 / 02 / 97 \\
10 / 07 / 97 \\
10 / 08 / 97 \\
07 / 01 / 97 \\
07 / 01 / 97 \\
07 / 02 / 97 \\
07 / 02 / 97 \\
10 / 01 / 97 \\
10 / 02 / 97 \\
10 / 07 / 97 \\
10 / 08 / 97 \\
07 / 01 / 97 \\
07 / 01 / 97 \\
07 / 02 / 97 \\
07 / 02 / 97 \\
10 / 01 / 97 \\
10 / 02 / 97 \\
10 / 07 / 97 \\
10 / 08 / 97 \\
07 / 01 / 97 \\
07 / 01 / 97 \\
07 / 02 / 97 \\
07 / 02 / 97 \\
10 / 01 / 97 \\
10 / 02 / 97\end{array}$ & 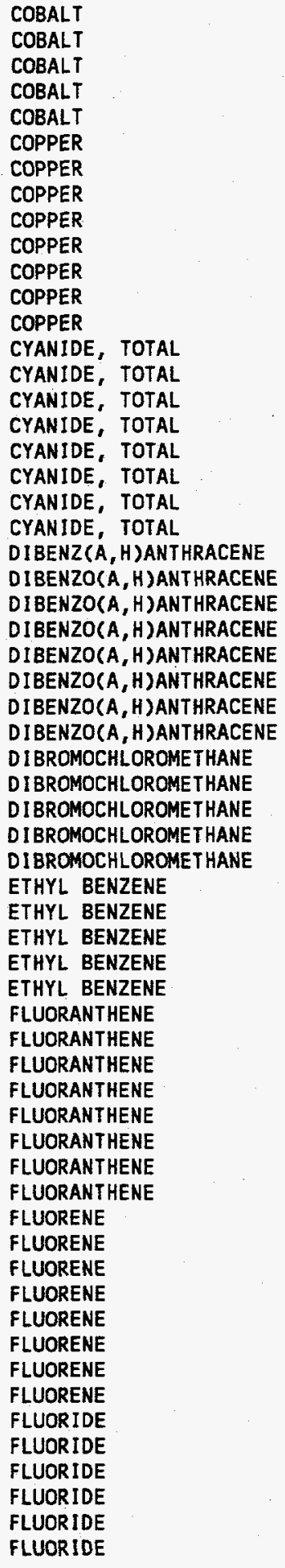 & 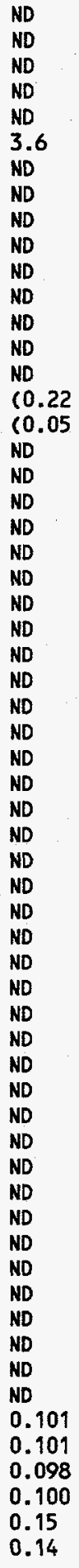 & $\begin{array}{l}0.6 \\
2.0 \\
2.0 \\
2.0 \\
2.0 \\
0.6 \\
0.6 \\
0.6 \\
0.6 \\
2.0 \\
2.0 \\
2.0 \\
2.0 \\
2.10 \\
2.10 \\
2.10 \\
2.10 \\
4.0 \\
4.0 \\
4.0 \\
4.0 \\
0.5 \\
1.00 \\
1.00 \\
1.00 \\
1.00 \\
1.00 \\
0.5 \\
0.0 \\
0.0 \\
0.0 \\
0.0 \\
0.0 \\
0 \\
0.0 \\
10 \\
10 \\
10 \\
10 \\
10 \\
10 \\
10 \\
10 \\
10 \\
10 \\
10 \\
10 \\
10 \\
10 \\
10 \\
1.00 \\
1.00 \\
1.00 \\
1.00 \\
1.00 \\
0.0 \\
0\end{array}$ & 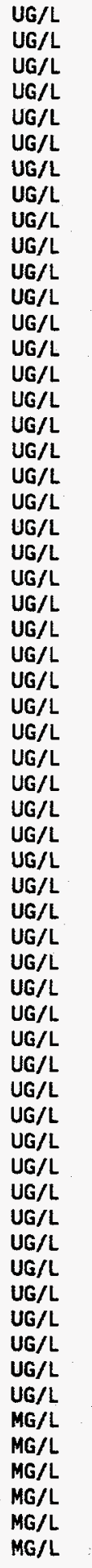 & $\begin{array}{l}\text { EPA } 6010 A \\
\text { EPA CLP } \\
\text { EPA } 6010 A \\
\text { EPA } 6010 A \\
\text { EPA } 6010 A \\
\text { EPA } 6010 A \\
\text { EPA } 6010 A \\
\text { EPA } 6010 A \\
\text { EPA } 6010 A \\
\text { EPA CLP } \\
\text { EPA } 6010 A \\
\text { EPA } 6010 A \\
\text { EPA } 6010 A \\
\text { EPA CLP } \\
\text { EPA CLP } \\
\text { EPA CLP } \\
\text { EPA CLP } \\
\text { EPA CLP } \\
\text { EPA CLP } \\
\text { EPA CLP } \\
\text { EPA CLP } \\
\text { SW846 } 8310 \\
\text { EPA } 8270 A \\
\text { EPA } 8270 A \\
\text { EPA } 8270 A \\
\text { EPA } 8270 A \\
\text { EPA } 8310 \\
\text { EPA } 8310 \\
\text { EPA } 8310 \\
\text { EPA CLP } \\
\text { EPA CLP } \\
\text { EPA CLP } \\
\text { EPA CLP } \\
\text { EPA CLP } \\
\text { EPA CLP } \\
\text { EPA CLP } \\
\text { EPA CLP } \\
\text { EPA CLP } \\
\text { EPA CLP } \\
\text { EPA } 8270 A \\
\text { EPA } 8270 A \\
\text { EPA } 8270 A \\
\text { EPA } 8270 A \\
\text { EPA } 8310 \\
\text { EPA } 8310 \\
\text { EPA } 8310 \\
\text { SW8 } 868310 \\
\text { EPA } 8270 A \\
\text { EPA } 8270 A \\
\text { EPA } 8270 A \\
\text { EPA } 8270 A \\
\text { EPA } 8310 \\
\text { EPA } 8310 \\
\text { EPA } 8310 \\
\text { SW846 } 8310 \\
\text { EPA } 300.0 \\
\text { EPA } 300.0 \\
\text { EPA } 300.0 \\
\text { EPA } 340.1 \\
\text { EPA } 340\end{array}$ \\
\hline
\end{tabular}




\begin{tabular}{|c|c|c|c|c|c|c|}
\hline WSSRAP_ID & DATE_SAM & PARAMETER & CONC & $\mathrm{DL}$ & UNITS & METHOD \\
\hline 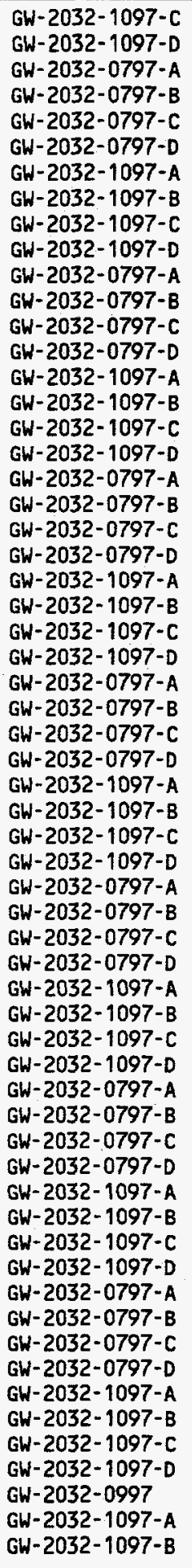 & $\begin{array}{l}10 / 07 / 97 \\
10 / 08 / 97 \\
07 / 01 / 97 \\
07 / 01 / 97 \\
07 / 02 / 97 \\
07 / 02 / 97 \\
10 / 01 / 97 \\
10 / 02 / 97 \\
10 / 07 / 97 \\
10 / 08 / 97 \\
07 / 01 / 97 \\
07 / 01 / 97 \\
07 / 02 / 97 \\
07 / 02 / 97 \\
10 / 01 / 97 \\
10 / 02 / 97 \\
10 / 07 / 97 \\
10 / 08 / 97 \\
07 / 01 / 97 \\
07 / 01 / 97 \\
07 / 02 / 97 \\
07 / 02 / 97 \\
10 / 01 / 97 \\
10 / 02 / 97 \\
10 / 07 / 97 \\
10 / 08 / 97 \\
07 / 01 / 97 \\
07 / 01 / 97 \\
07 / 02 / 97 \\
07 / 02 / 97 \\
10 / 01 / 97 \\
10 / 02 / 97 \\
10 / 07 / 97 \\
10 / 08 / 97 \\
07 / 01 / 97 \\
07 / 01 / 97 \\
07 / 02 / 97 \\
07 / 02 / 97 \\
10 / 01 / 97 \\
10 / 02 / 97 \\
10 / 07 / 97 \\
10 / 08 / 97 \\
07 / 01 / 97 \\
07 / 01 / 97 \\
07 / 02 / 97 \\
07 / 02 / 97 \\
10 / 01 / 97 \\
10 / 02 / 97 \\
10 / 07 / 97 \\
10 / 08 / 97 \\
07 / 01 / 97 \\
07 / 01 / 97 \\
07 / 02 / 97 \\
07 / 02 / 97 \\
10 / 01 / 97 \\
10 / 02 / 97 \\
10 / 07 / 97 \\
10 / 08 / 97 \\
09 / 11 / 97 \\
10 / 01 / 97 \\
10 / 02 / 97 \\
\end{array}$ & $\begin{array}{l}\text { FLUORIDE } \\
\text { FLUORIDE } \\
\text { INDENO(1,2,3-CD)PYRENE } \\
\text { INDENO(1,2,3-CD)PYRENE } \\
\text { INDENO(1,2,3-CD)PYRENE } \\
\text { INDENO(1,2,3-CD)PYRENE } \\
\text { INDENO(1,2,3-CD)PYRENE } \\
\text { INDENO(1,2,3-CD)PYRENE } \\
\text { INDENO(1,2,3-CD)PYRENE } \\
\text { INDENO(1,2,3-CD)PYRENE } \\
\text { IRON } \\
\text { IRON } \\
\text { IRON } \\
\text { IRON } \\
\text { IRON } \\
\text { IRON } \\
\text { IRON } \\
\text { IRON } \\
\text { LEAD } \\
\text { LEAD } \\
\text { LEAD } \\
\text { LEAD } \\
\text { LEAD } \\
\text { LEAD } \\
\text { LEAD } \\
\text { LEAD } \\
\text { LITHIUM } \\
\text { LITHIUM } \\
\text { LITHIUM } \\
\text { LITHIUM } \\
\text { LITHIUM } \\
\text { LITHIUM } \\
\text { LITHIUM } \\
\text { LITHIUM } \\
\text { MAGNESIUM } \\
\text { MAGNESIUM } \\
\text { MAGNESIUM } \\
\text { MAGNESIUM } \\
\text { MAGNESIUM } \\
\text { MAGNESIUM } \\
\text { MAGNESIUM } \\
\text { MAGNESIUM } \\
\text { MANGANESE } \\
\text { MANGANESE } \\
\text { MANGANESE } \\
\text { MANGANESE } \\
\text { MANGANESE } \\
\text { MANGANESE } \\
\text { MANGANESE } \\
\text { MANGANESE } \\
\text { MERCURY } \\
\text { MERCURY } \\
\text { MERCURY } \\
\text { MERCURY } \\
\text { MERCURY } \\
\text { MERCURY } \\
\text { MERCURY } \\
\text { MERCURY } \\
\text { METHYLENE CHLORIDE } \\
\text { METHYLENE CHLORIDE } \\
\text { METHYLENE CHLORIDE } \\
\text { MEN }\end{array}$ & $\begin{array}{l}0.12 \\
0.14 \\
\text { ND } \\
\text { ND } \\
\text { ND } \\
\text { ND } \\
\text { ND } \\
\text { ND } \\
\text { ND } \\
\text { ND } \\
87.3 \\
156 \\
88.5 \\
111 \\
424 \\
692 \\
282 \\
300 \\
\text { ND } \\
\text { ND } \\
\text { ND } \\
\text { ND } \\
2.4 \\
\text { ND } \\
2.8 \\
\text { ND } \\
14.8 \\
22.7 \\
\text { ND } \\
22.5 \\
6.4 \\
8.2 \\
6.1 \\
7.8 \\
45100 \\
41600 \\
40600 \\
39600 \\
31300 \\
32400 \\
29900 \\
32100 \\
11.8 \\
15.8 \\
6.8 \\
10.1 \\
15.8 \\
21.2 \\
9.2 \\
10.7 \\
\text { ND } \\
0.54 \\
0.25 \\
0.18 \\
\text { ND } \\
\text { ND } \\
\text { ND } \\
\text { ND } \\
\text { ND } \\
(7) \\
19 \\
19\end{array}$ & $\begin{array}{l}0.05 \\
0.05 \\
1.00 \\
1.00 \\
1.00 \\
1.00 \\
0.5 \\
0.5 \\
0.5 \\
0.5 \\
2.0 \\
2.0 \\
2.0 \\
2.0 \\
61.0 \\
61.0 \\
61.0 \\
61.0 \\
0.7 \\
0.7 \\
0.7 \\
0.7 \\
2.0 \\
2.0 \\
2.0 \\
2.0 \\
11.0 \\
11.0 \\
11.0 \\
11.0 \\
2.0 \\
2.0 \\
2.0 \\
2.0 \\
3.3 \\
3.3 \\
3.3 \\
3.3 \\
28.0 \\
28.0 \\
28.0 \\
28.0 \\
0.3 \\
0.3 \\
0.3 \\
0.3 \\
2.0 \\
2.0 \\
2.0 \\
2.0 \\
0.1 \\
0.1 \\
0.1 \\
0.1 \\
0.10 \\
0.10 \\
0.10 \\
0.10 \\
10 \\
10 \\
10 \\
\end{array}$ & 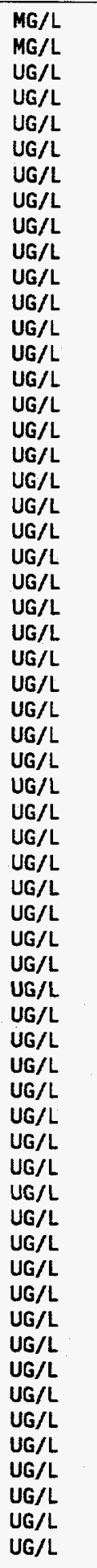 & $\begin{array}{l}\text { EPA } 340.0 \\
\text { EPA } 340 \\
\text { EPA } 8270 A \\
\text { EPA } 8270 A \\
\text { EPA } 8270 A \\
\text { EPA } 8270 A \\
\text { EPA } 8310 \\
\text { EPA } 8310 \\
\text { EPA } 8310 \\
\text { SW } 8668310 \\
\text { EPA } 6010 A \\
\text { EPA } 6010 A \\
\text { EPA } 6010 A \\
\text { EPA } 6010 A \\
\text { EPA CLP } \\
\text { EPA } 6010 A \\
\text { EPA } 6010 A \\
\text { EPA } 6010 A \\
\text { EPA } 6010 A \\
\text { EPA } 6010 A \\
\text { EPA } 6010 A \\
\text { EPA } 6010 A \\
\text { EPA CLP } \\
\text { EPA } 6010 A \\
\text { EPA } 6010 A \\
\text { EPA } 6010 A \\
\text { EPA } 6010 A \\
\text { EPA } 6010 A \\
\text { EPA } 6010 A \\
\text { EPA } 6010 A \\
\text { EPA CLP } \\
\text { EPA } 6010 A \\
\text { EPA } 6010 A \\
\text { EPA } 6010 A \\
\text { EPA } 6010 A \\
\text { EPA } 6010 A \\
\text { EPA } 6010 A \\
\text { EPA } 6010 A \\
\text { EPA CLP } \\
\text { EPA } 6010 A \\
\text { EPA } 6010 A \\
\text { EPA } 6010 A \\
\text { EPA } 6010 A \\
\text { EPA } 6010 A \\
\text { EPA } 6010 A \\
\text { EPA } 6010 A \\
\text { EPA CLP } \\
\text { EPA } 6010 A \\
\text { EPA } 6010 A \\
\text { EPA } 6010 A \\
\text { EPA } 7470 A \\
\text { EPA } 7470 A \\
\text { EPA } 7470 A \\
\text { EPA } 7470 A \\
\text { EPA CLP } \\
\text { EPA } 7470 A \\
\text { EPA } 7470 A \\
\text { EPA } 7470 \\
\text { EPA } C L P \\
\text { EPA CLP } \\
\text { EPA CLP }\end{array}$ \\
\hline
\end{tabular}




\begin{tabular}{|c|c|c|c|c|c|c|}
\hline WSSR & DATE_SAM & \multicolumn{2}{|l|}{ PARAMETER } & $\mathrm{DL}$ & UNITS & METHOD \\
\hline 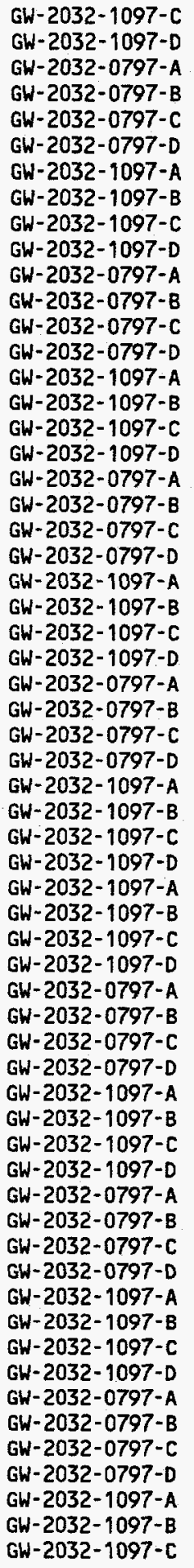 & $\begin{array}{l}10 / 07 / 97 \\
10 / 08 / 97 \\
07 / 01 / 97 \\
07 / 01 / 97 \\
07 / 02 / 97 \\
07 / 02 / 97 \\
10 / 01 / 97 \\
10 / 02 / 97 \\
10 / 07 / 97 \\
10 / 08 / 97 \\
07 / 01 / 97 \\
07 / 01 / 97 \\
07 / 02 / 97 \\
07 / 02 / 97 \\
10 / 01 / 97 \\
10 / 02 / 97 \\
10 / 07 / 97 \\
10 / 08 / 97 \\
07 / 01 / 97 \\
07 / 01 / 97 \\
07 / 02 / 97 \\
07 / 02 / 97 \\
10 / 01 / 97 \\
10 / 02 / 97 \\
10 / 07 / 97 \\
10 / 08 / 97 \\
07 / 01 / 97 \\
07 / 01 / 97 \\
07 / 02 / 97 \\
07 / 02 / 97 \\
10 / 01 / 97 \\
10 / 02 / 97 \\
10 / 07 / 97 \\
10 / 08 / 97 \\
10 / 01 / 97 \\
10 / 02 / 97 \\
10 / 07 / 97 \\
10 / 08 / 97 \\
07 / 01 / 97 \\
07 / 01 / 97 \\
07 / 02 / 97 \\
07 / 02 / 97 \\
10 / 011 / 97 \\
10 / 02 / 97 \\
10 / 07 / 97 \\
10 / 08 / 97 \\
07 / 01 / 97 \\
07 / 01 / 97 \\
07 / 02 / 97 \\
07 / 02 / 97 \\
10 / 01 / 97 \\
10 / 02 / 97 \\
10 / 07 / 97 \\
10 / 08 / 97 \\
07 / 01 / 97 \\
07 / 01 / 97 \\
07 / 02 / 97 \\
07 / 02 / 97 \\
10 / 01 / 97 \\
10 / 02 / 97 \\
10 / 07 / 97\end{array}$ & 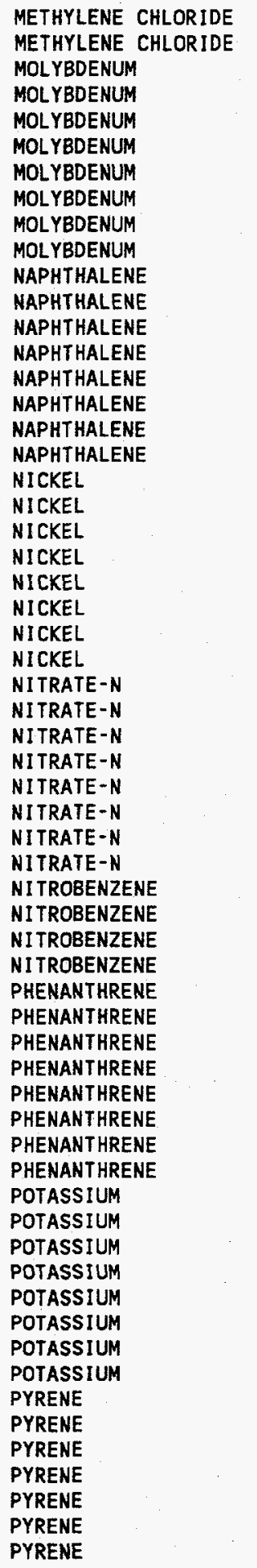 & $\begin{array}{l}\text { (5) } \\
\text { ND } \\
1.4 \\
0.88 \\
0.91 \\
1.9 \\
7.1 \\
\text { ND } \\
\text { ND } \\
\text { ND } \\
\text { ND } \\
\text { ND } \\
\text { ND } \\
\text { ND } \\
\text { ND } \\
\text { ND } \\
\text { ND } \\
\text { ND } \\
2.3 \\
2.3 \\
2.1 \\
5.5 \\
5.2 \\
\text { ND } \\
4.4 \\
3.6 \\
58.8 \\
76.8 \\
74.8 \\
58.2 \\
10.9 \\
21.1 \\
19.4 \\
24.9 \\
\text { ND } \\
\text { ND } \\
\text { ND } \\
\text { ND } \\
\text { ND } \\
\text { ND } \\
\text { ND } \\
\text { ND } \\
\text { ND } \\
\text { ND } \\
\text { ND } \\
\text { ND } \\
2660 \\
2840 \\
2800 \\
2400 \\
1510 \\
1880 \\
1910 \\
2250 \\
\text { ND } \\
\text { ND } \\
\text { ND } \\
\text { ND } \\
\text { ND } \\
\text { ND } \\
\text { ND }\end{array}$ & $\begin{array}{l}10 \\
10 \\
0.7 \\
0.7 \\
0.7 \\
0.7 \\
7.0 \\
7.0 \\
7.0 \\
7.0 \\
1.00 \\
1.00 \\
1.00 \\
1.00 \\
0.5 \\
0.5 \\
0.5 \\
0.5 \\
1.0 \\
1.0 \\
1.0 \\
1.0 \\
2.0 \\
4.7 \\
2.0 \\
2.0 \\
0.280 \\
0.280 \\
0.280 \\
0.280 \\
0.200 \\
0.400 \\
0.400 \\
0.400 \\
0.030 \\
0.030 \\
0.030 \\
0.030 \\
1.00 \\
1.00 \\
1.00 \\
1.00 \\
0.5 \\
0.5 \\
0.5 \\
0.5 \\
11.0 \\
11.0 \\
11.0 \\
11.0 \\
94.0 \\
94.0 \\
69.0 \\
94.0 \\
1.00 \\
1.00 \\
1.00 \\
1.00 \\
0.5 \\
0.5 \\
0.5\end{array}$ & $\begin{array}{l}\text { UG/L } \\
U G / L \\
U G / L \\
U G / L \\
U G / L \\
U G / L \\
U G / L \\
U G / L \\
U G / L \\
U G / L \\
U G / L \\
U G / L \\
U G / L \\
U G / L \\
U G / L \\
U G / L \\
U G / L \\
U G / L \\
U G / L \\
U G / L \\
U G / L \\
U G / L \\
U G / L \\
U G / L \\
U G / L \\
U G / L \\
M G / L \\
M G / L \\
M G / L \\
M G / L \\
M G / L \\
M G / L \\
M G / L \\
M G / L \\
U G / L \\
U G / L \\
U G / L \\
U G / L \\
U G / L \\
U G / L \\
U G / L \\
U G / L \\
U G / L \\
U G / L \\
U G / L \\
U G / L \\
U G / L \\
U G / L \\
U G / L \\
U G / L \\
U G / L \\
U G / L \\
U G / L \\
U G / L \\
U G / L \\
U G / L \\
U G / L \\
U G / L \\
U G / L \\
U G / L \\
U,\end{array}$ & 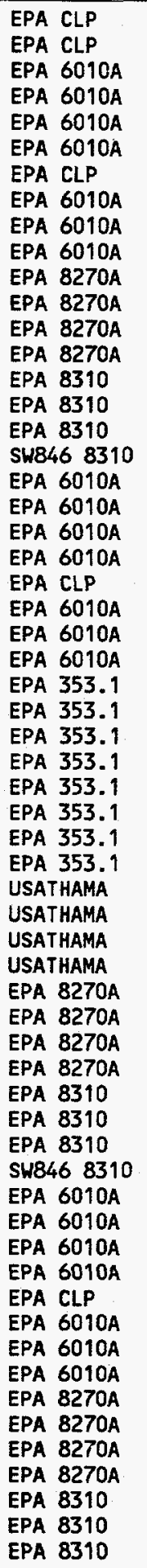 \\
\hline
\end{tabular}




\begin{tabular}{|c|c|c|c|c|c|c|c|}
\hline USSRAP_ID & DATE_SAM & PARAMETER & CONC & $\mathrm{DL}$ & UNITS & METHOD & \\
\hline 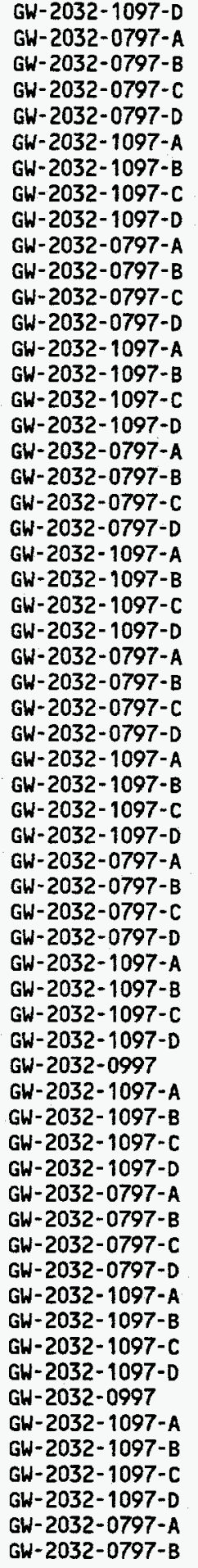 & $\begin{array}{l}10 / 08 / 97 \\
07 / 01 / 97 \\
07 / 01 / 97 \\
07 / 02 / 97 \\
07 / 02 / 97 \\
10 / 01 / 97 \\
10 / 02 / 97 \\
10 / 07 / 97 \\
10 / 08 / 97 \\
07 / 01 / 97 \\
07 / 01 / 97 \\
07 / 02 / 97 \\
07 / 02 / 97 \\
10 / 01 / 97 \\
10 / 02 / 97 \\
10 / 07 / 97 \\
10 / 08 / 97 \\
07 / 01 / 97 \\
07 / 01 / 97 \\
07 / 02 / 97 \\
07 / 02 / 97 \\
10 / 01 / 97 \\
10 / 02 / 97 \\
10 / 07 / 97 \\
10 / 08 / 97 \\
07 / 01 / 97 \\
07 / 01 / 97 \\
07 / 02 / 97 \\
07 / 02 / 97 \\
10 / 01 / 97 \\
10 / 02 / 97 \\
10 / 07 / 97 \\
10 / 08 / 97 \\
07 / 01 / 97 \\
07 / 01 / 97 \\
07 / 02 / 97 \\
07 / 02 / 97 \\
10 / 01 / 97 \\
10 / 02 / 97 \\
10 / 07 / 97 \\
10 / 08 / 97 \\
09 / 11 / 97 \\
10 / 01 / 97 \\
10 / 02 / 97 \\
10 / 07 / 97 \\
10 / 08 / 97 \\
07 / 01 / 97 \\
07 / 01 / 97 \\
07 / 02 / 97 \\
07 / 02 / 97 \\
10 / 01 / 97 \\
10 / 02 / 97 \\
10 / 07 / 97 \\
10 / 08 / 97 \\
09 / 11 / 97 \\
10 / 01 / 97 \\
10 / 02 / 97 \\
10 / 07 / 97 \\
10 / 08 / 97 \\
07 / 01 / 97 \\
07 / 01 / 97\end{array}$ & $\begin{array}{l}\text { PYRENE } \\
\text { RADIUM-226 } \\
\text { RADIUM-226 } \\
\text { RADIUM-226 } \\
\text { RADIUM-226 } \\
\text { RADIUM-226 } \\
\text { RADIUM-226 } \\
\text { RADIUM-226 } \\
\text { RADIUM-226 } \\
\text { RADIUM-228 } \\
\text { RADIUM-228 } \\
\text { RADIUM-228 } \\
\text { RADIUM-228 } \\
\text { RADIUM-228 } \\
\text { RADIUM-228 } \\
\text { RADIUM-228 } \\
\text { RADIUM-228 } \\
\text { SELENIUM } \\
\text { SELENIUM } \\
\text { SELENIUM } \\
\text { SELENIUM } \\
\text { SELENIUM } \\
\text { SELENIUM } \\
\text { SELENIUM } \\
\text { SELENIUM } \\
\text { SILVER } \\
\text { SILVER } \\
\text { SILVER } \\
\text { SILVER } \\
\text { SILVER } \\
\text { SILVER } \\
\text { SILVER } \\
\text { SILVER } \\
\text { SODIUM } \\
\text { SODIUM } \\
\text { SODIUM } \\
\text { SODIUM } \\
\text { SODIUM } \\
\text { SODIUM } \\
\text { SODIUM } \\
\text { SODIUM } \\
\text { STYRENE } \\
\text { STYRENE } \\
\text { STYRENE } \\
\text { STYRENE } \\
\text { STYRENE } \\
\text { SULFATE } \\
\text { SULFATE } \\
\text { SULFATE } \\
\text { SULFATE } \\
\text { SULFATE } \\
\text { SULFATE } \\
\text { SULFATE } \\
\text { SULFATE } \\
\text { TETRACHLOROETHENE } \\
\text { TETRACHLOROETHENE } \\
\text { TETRACHLOROETHENE } \\
\text { TETRACHLOROETHENE } \\
\text { TETRACHLOROETHENE } \\
\text { THALLIUM } \\
\text { THALLIUM } \\
\end{array}$ & $\begin{array}{l}\text { ND } \\
\text { ND } \\
0.337 \\
0.310 \\
60.28 \\
0.150 \\
60.03 \\
0.400 \\
60.28 \\
60.48 \\
1.06 \\
0.29 \\
4.37 \\
60.56 \\
1.02 \\
11.03 \\
C 0.56 \\
4.0 \\
3.7 \\
4.4 \\
3.0 \\
\text { ND } \\
\text { ND } \\
\text { ND } \\
\text { ND } \\
\text { ND } \\
4.2 \\
1.3 \\
1.8 \\
\text { ND } \\
\text { ND } \\
\text { ND } \\
\text { ND } \\
50300 \\
52300 \\
52700 \\
43400 \\
16900 \\
25200 \\
19400 \\
25500 \\
\text { ND } \\
\text { ND } \\
\text { ND } \\
\text { ND } \\
\text { ND } \\
37.7 \\
42.7 \\
43.4 \\
39.8 \\
21.2 \\
27.3 \\
22.7 \\
28.4 \\
\text { ND } \\
\text { ND } \\
\text { ND } \\
\text { ND } \\
\text { ND } \\
2.5 \\
2.9\end{array}$ & 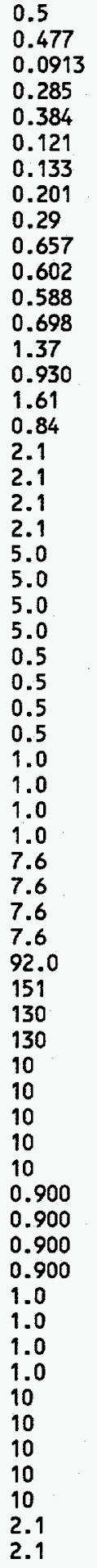 & $\begin{array}{l}U G / L \\
P C I / L \\
P C I / L \\
P C I / L \\
P C I / L \\
P C I / L \\
P C I / L \\
P C I / L \\
P C I / L \\
P C I / L \\
P C I / L \\
P C I / L \\
P C I / L \\
P C I / L \\
P C I / L \\
P C I / L \\
P C I / L \\
U G / L \\
U G / L \\
U G / L \\
U G / L \\
U G / L \\
U G / L \\
U G / L \\
U G / L \\
U G / L \\
U G / L \\
U G / L \\
U G / L \\
U G / L \\
U G / L \\
U G / L \\
U G / L \\
U G / L \\
U G / L \\
U G / L \\
U G / L \\
U G / L \\
U G / L \\
U G / L \\
U G / L \\
U G / L \\
U G / L \\
U G / L \\
U G / L \\
U G / L \\
M G / L \\
M G / L \\
M G / L \\
M G / L \\
M G / L \\
M G / L \\
M G / L \\
M G / L \\
U G / L \\
U G / L \\
U G / L \\
U G / L \\
U G / L \\
U G / L \\
U G / L \\
\end{array}$ & 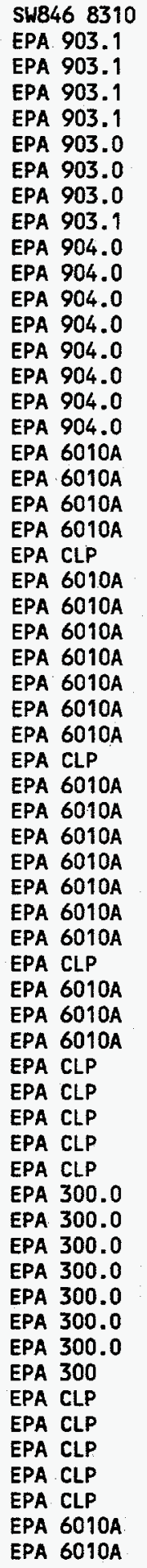 & r \\
\hline
\end{tabular}




\begin{tabular}{|c|c|c|c|c|c|c|}
\hline WSSRAP_ID & DATE_SAM & PARAMETER & CONC & $\mathrm{DL}$ & UNITS & METHOD \\
\hline $\begin{array}{l}\text { GW-2032-0797-C } \\
\text { GW-2032-0797-D } \\
\text { GW-2032-1097-A } \\
\text { GW-2032-1097-B } \\
\text { GW-2032-1097-C } \\
\text { GW-2032-1097-D } \\
\text { GW-2032-0797-A } \\
\text { GW-2032-0797-B } \\
\text { GW-2032-0797-C } \\
\text { GW-2032-0797-D } \\
G W-2032-1097-A \\
G W-2032-1097-B \\
G W-2032-1097-C \\
G W-2032-1097-D \\
G W-2032-0797-A \\
G W-2032-0797-B \\
G W-2032-0797-C \\
G W-2032-0797-D \\
G W-2032-1097-A \\
G W-2032-1097-B \\
G W-2032-1097-C \\
G W-2032-1097-D \\
G W-2032-0797-A \\
G W-2032-0797-B \\
G W-2032-0797-C \\
G W-2032-0797-D \\
G W-2032-1097-A \\
G W-2032-1097-B \\
G W-2032-1097-C \\
G W-2032-1097-D \\
G W-2032-0997 \\
G W-2032-1097-A \\
G W-2032-1097-B \\
G W-2032-1097-C \\
G W-2032-1097-D \\
G W-2032-0797-A \\
G W-2032-0797-B \\
G W-2032-0797-C \\
G W-2032-0797-D \\
G W-2032-1097-A \\
G W-2032-1097-B \\
G W-2032-1097-C \\
G W-2032-1097-D \\
G W-2032-0797-A \\
G W-2032-0797-B \\
G W-2032-0797-C \\
G W-2032-0797-D \\
G W-2032-1097-A \\
G W-2032-1097-B \\
G W-2032-1097-C \\
G W-2032-1097-D \\
G W-2032-0797-A \\
G W-2032-0797-B \\
G W-2032-0797-C \\
G W-2032-0797-D \\
G W-2032-1097-A \\
G W-2032-1097-B \\
G W-2032-1097-C \\
G W-2032-1097-D \\
G W-2032-0997 \\
G W-2032-1097-A\end{array}$ & $\begin{array}{l}07 / 02 / 97 \\
07 / 02 / 97 \\
10 / 01 / 97 \\
10 / 02 / 97 \\
10 / 07 / 97 \\
10 / 08 / 97 \\
07 / 01 / 97 \\
07 / 01 / 97 \\
07 / 02 / 97 \\
07 / 02 / 97 \\
10 / 01 / 97 \\
10 / 02 / 97 \\
10 / 07 / 97 \\
10 / 08 / 97 \\
07 / 01 / 97 \\
07 / 01 / 97 \\
07 / 02 / 97 \\
07 / 02 / 97 \\
10 / 01 / 97 \\
10 / 02 / 97 \\
10 / 07 / 97 \\
10 / 08 / 97 \\
07 / 01 / 97 \\
07 / 01 / 97 \\
07 / 02 / 97 \\
07 / 02 / 97 \\
10 / 01 / 97 \\
10 / 02 / 97 \\
10 / 07 / 97 \\
10 / 08 / 97 \\
09 / 11 / 97 \\
10 / 01 / 97 \\
10 / 02 / 97 \\
10 / 07 / 97 \\
10 / 08 / 97 \\
07 / 01 / 97 \\
07 / 01 / 97 \\
07 / 02 / 97 \\
07 / 02 / 97 \\
10 / 01 / 97 \\
10 / 02 / 97 \\
10 / 07 / 97 \\
10 / 08 / 97 \\
07 / 01 / 97 \\
07 / 01 / 97 \\
07 / 02 / 97 \\
07 / 02 / 97 \\
10 / 01 / 97 \\
10 / 02 / 97 \\
10 / 07 / 97 \\
10 / 08 / 97 \\
07 / 01 / 97 \\
07 / 01 / 97 \\
07 / 02 / 97 \\
07 / 02 / 97 \\
10 / 01 / 97 \\
10 / 02 / 97 \\
10 / 07 / 97 \\
10 / 08 / 97 \\
09 / 11 / 97 \\
10 / 01 / 97\end{array}$ & 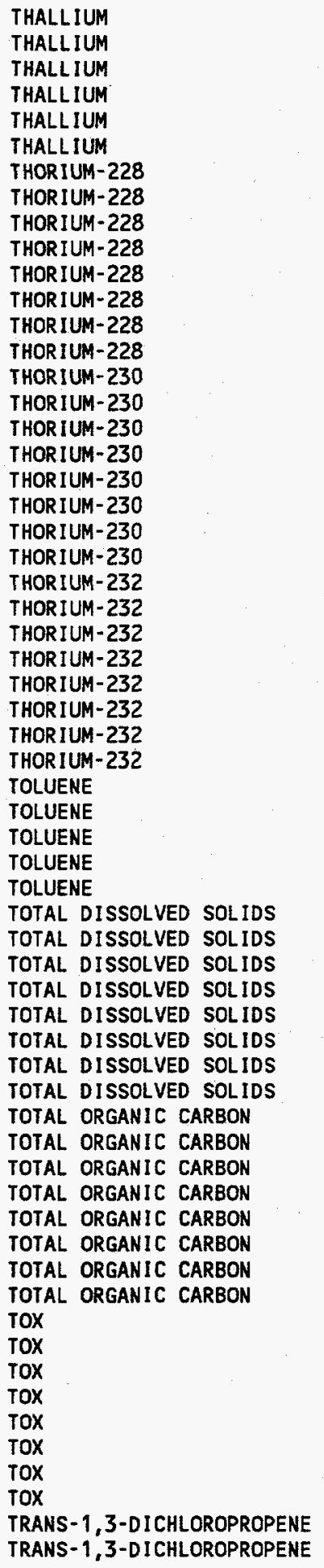 & $\begin{array}{l}2.6 \\
\text { ND } \\
\text { ND } \\
\text { ND } \\
\text { ND } \\
\text { ND } \\
\text { ND } \\
\text { ND } \\
\text { ND } \\
\text { ND } \\
\text { ND } \\
C 0.23 \\
C 0.17 \\
C 0.04 \\
C 0.06 \\
C 0.09 \\
C 0.09 \\
C 0.04 \\
0.318 \\
C 0.18 \\
C 0.08 \\
0.076 \\
C 0.02 \\
\text { ND } \\
C 0.01 \\
C 0.00 \\
\text { ND } \\
C 0.04 \\
C 0.05 \\
C 0.03 \\
\text { ND } \\
\text { ND } \\
\text { ND } \\
\text { ND } \\
\text { ND } \\
680 \\
812 \\
785 \\
680 \\
442 \\
562 \\
476 \\
528 \\
2.40 \\
2.10 \\
2.23 \\
2.26 \\
14.4 \\
6.03 \\
30.2 \\
25.8 \\
0.009 \\
0.010 \\
0.016 \\
0.009 \\
0.024 \\
0.031 \\
0.008 \\
0.024 \\
\text { ND } \\
0.03\end{array}$ & 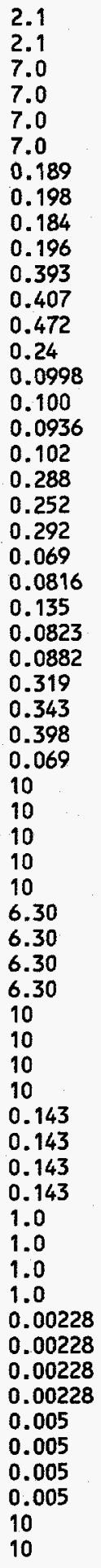 & 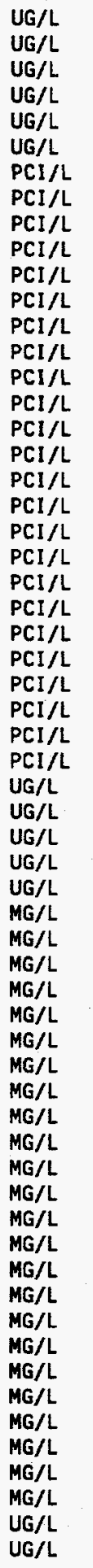 & 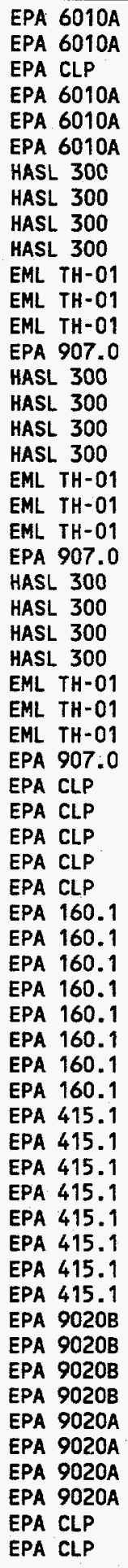 \\
\hline
\end{tabular}




\begin{tabular}{|c|c|c|c|c|c|c|}
\hline WSSRAP_ID & DATE_SAM & PARAMETER & CONC & $\mathrm{DL}$ & UNITS & METHOD \\
\hline 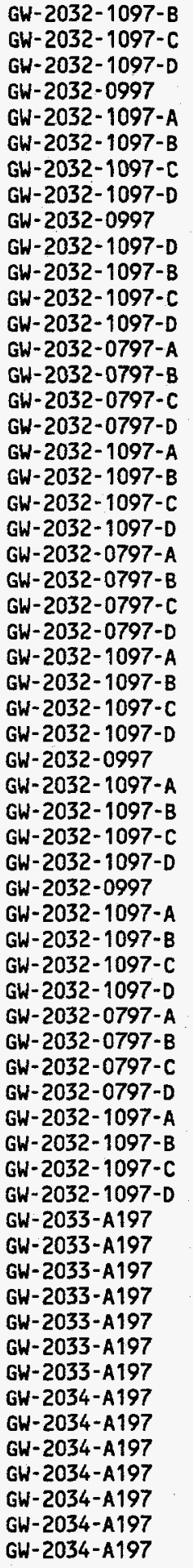 & $\begin{array}{l}10 / 02 / 97 \\
10 / 07 / 97 \\
10 / 08 / 97 \\
09 / 11 / 97 \\
10 / 01 / 97 \\
10 / 02 / 97 \\
10 / 07 / 97 \\
10 / 08 / 97 \\
09 / 11 / 97 \\
10 / 01 / 97 \\
10 / 02 / 97 \\
10 / 07 / 97 \\
10 / 08 / 97 \\
07 / 01 / 97 \\
07 / 01 / 97 \\
07 / 02 / 97 \\
07 / 02 / 97 \\
10 / 01 / 97 \\
10 / 02 / 97 \\
10 / 07 / 97 \\
10 / 08 / 97 \\
07 / 01 / 97 \\
07 / 01 / 97 \\
07 / 02 / 97 \\
07 / 02 / 97 \\
10 / 01 / 97 \\
10 / 02 / 97 \\
10 / 07 / 97 \\
10 / 08 / 97 \\
09 / 11 / 97 \\
10 / 01 / 97 \\
10 / 02 / 97 \\
10 / 07 / 97 \\
10 / 08 / 97 \\
09 / 11 / 97 \\
10 / 01 / 97 \\
10 / 02 / 97 \\
10 / 07 / 97 \\
10 / 08 / 97 \\
07 / 01 / 97 \\
07 / 01 / 97 \\
07 / 02 / 97 \\
07 / 02 / 97 \\
10 / 01 / 97 \\
10 / 02 / 97 \\
10 / 07 / 97 \\
10 / 08 / 97 \\
08 / 25 / 97 \\
08 / 25 / 97 \\
08 / 25 / 97 \\
08 / 25 / 97 \\
08 / 25 / 97 \\
08 / 25 / 97 \\
08 / 25 / 97 \\
08 / 25 / 97 \\
08 / 25 / 97 \\
08 / 25 / 97 \\
08 / 25 / 97 \\
08 / 25 / 97 \\
08 / 25 / 97 \\
08 / 25 / 97\end{array}$ & 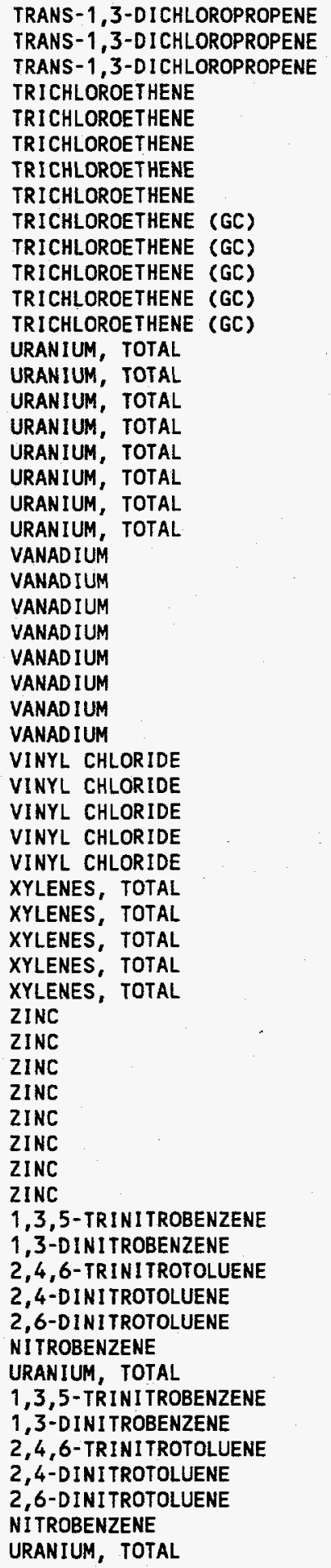 & $\begin{array}{l}\text { ND } \\
\text { ND } \\
\text { ND } \\
\text { ND } \\
\text { ND } \\
\text { ND } \\
\text { ND } \\
\text { ND } \\
\text { ND } \\
\text { ND } \\
\text { ND } \\
\text { ND } \\
\text { ND } \\
4.22 \\
4.27 \\
4.35 \\
4.28 \\
3.00 \\
3.38 \\
2.93 \\
3.17 \\
0.50 \\
\text { ND } \\
\text { ND } \\
\text { ND } \\
\text { ND } \\
\text { ND } \\
\text { ND } \\
\text { ND } \\
\text { ND } \\
\text { ND } \\
\text { ND } \\
\text { ND } \\
\text { ND } \\
\text { ND } \\
\text { ND } \\
\text { ND } \\
\text { ND } \\
\text { ND } \\
1.4 \\
4.2 \\
6.4 \\
4.5 \\
23.8 \\
20.3 \\
14.2 \\
12.0 \\
5.3 \\
\text { ND } \\
1.4 \\
0.12 \\
1.5 \\
\text { ND } \\
1.11 \\
\text { ND } \\
\text { ND } \\
\text { ND } \\
\text { ND } \\
\text { ND } \\
\text { ND } \\
2.90\end{array}$ & $\begin{array}{l}10 \\
10 \\
10 \\
10 \\
10 \\
10 \\
10 \\
10 \\
10 \\
10 \\
1.0 \\
1.0 \\
1.0 \\
1.0 \\
1.0 \\
1.0 \\
0 \\
0.064 \\
0 \\
0.064 \\
0 \\
0.0643 \\
0.0643 \\
0 \\
0.0203 \\
0.0203 \\
\mid\end{array}$ & 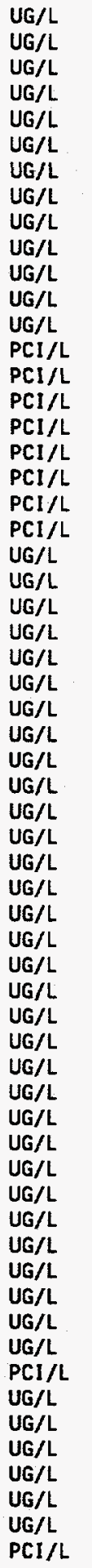 & $\begin{array}{l}\text { EPA CLP } \\
\text { EPA CLP } \\
\text { EPA CLP } \\
\text { EPA CLP } \\
\text { EPA CLP } \\
\text { EPA CLP } \\
\text { EPA CLP } \\
\text { EPA CLP } \\
\text { EPA } 601 \\
\text { EPA } 601 \\
\text { EPA } 601 \\
\text { EPA } 601 \\
\text { EPA } 601 \\
\text { ASTM } 5174-91 \\
\text { ASTM } 5174-91 \\
\text { ASTM } 5174-91 \\
\text { ASTM } 5174-91 \\
\text { ASTM } 5174-91 \\
\text { ASTM } 5174-91 \\
\text { ASTM } 5174-91 \\
\text { ASTM } 5174-91 \\
\text { EPA } 6010 A \\
\text { EPA } 6010 A \\
\text { EPA } 6010 A \\
\text { EPA } 6010 A \\
\text { EPA CLP } \\
\text { EPA } 6010 A \\
\text { EPA } 6010 A \\
\text { EPA } 6010 A \\
\text { EPA CLP } \\
\text { EPA CLP } \\
\text { EPA CLP } \\
\text { EPA CLP } \\
\text { EPA CLP } \\
\text { EPA CLP } \\
\text { EPA CLP } \\
\text { EPA CLP } \\
\text { EPA CLP } \\
\text { EPA CLP } \\
\text { EPA } 6010 A \\
\text { EPA } 6010 A \\
\text { EPA } 6010 A \\
\text { EPA } 6010 A \\
\text { EPA CLP } \\
\text { EPA } 6010 A \\
\text { EPA } 6010 A \\
\text { EPA } 6010 A \\
\text { USATHAMA } \\
\text { USATHAMA } \\
\text { USATHAMA } \\
\text { USATHAMA } \\
\text { USATHAMA } \\
\text { USATHAMA } \\
\text { ASTM } 5174-91 \\
\text { USATHAMA } \\
\text { USATHAMA } \\
\text { USATHAMA } \\
\text { USATHAMA } \\
\text { USATHAMA } \\
\text { USATHAMA } \\
\text { ASTM 5174-91 }\end{array}$ \\
\hline
\end{tabular}




\begin{tabular}{|c|c|c|c|c|c|c|}
\hline WSSRAP_ID & DATE_SAM & PARAMETER & CONC & $D L$ & UNITS & METHOD \\
\hline $\begin{array}{l}\text { GW-2035-0397 } \\
\text { GW-2035-0397 } \\
\text { GW-2035-0397 } \\
\text { GW-2035-0397 } \\
\text { GW-2035-0397 } \\
\text { GW-2035-0397 } \\
\text { GW-2036-0397 } \\
\text { GW-2036-0397 } \\
\text { GW-2036-0397 } \\
\text { GW-2036-0397 } \\
\text { GW-2036-0397 } \\
\text { GW-2036-0397 } \\
\text { GW-2037-0997 } \\
\text { GW-2037-1097 } \\
\text { GW-2037-0997 } \\
\text { GW-2037-1097 } \\
\text { GW-2037-0997 } \\
\text { GW-2037-1097 } \\
\text { GW-2037-0997 } \\
\text { GW-2037-1097 } \\
\text { GW-2037-0997 } \\
\text { GW-2037-1097 } \\
\text { GW-2037-0997 } \\
\text { GW-2037-1097 } \\
\text { GW-2037-0997 } \\
\text { GW-2037-1097 } \\
\text { GW-2037-0997 } \\
\text { GW-2037-1097 } \\
\text { GW-2037-0997 } \\
\text { GW-2037-1097 } \\
\text { GW-2037-0997 } \\
\text { GW-2037-1097 } \\
\text { GW-2037-0997 } \\
\text { GW-2037-1097 } \\
\text { GW-2037-0997 } \\
\text { GW-2037-1097 } \\
\text { GW-2037-0997 } \\
\text { GW-2037-0997 } \\
\text { GW-2037-0997 } \\
\text { GW-2037-1097 } \\
\text { GW-2037-0997 } \\
\text { GW-2037-1097 } \\
\text { GW-2037-0997 } \\
\text { GW-2037-1097 } \\
\text { GW-2037-0997 } \\
\text { GW-2037--1097 } \\
\text { GW-2037-09997 } \\
\text { GW-2037-0997 } \\
\text { GW-2037-1097 } \\
\text { GW-2037-0997 } \\
\text { GW-2037-2037-09997-1097 } \\
\text { GW-2037-0997 } \\
\text { GW-2037-1097 } \\
\text { GW-2037-0997 } \\
\text { GW-2037-1097 }\end{array}$ & $\begin{array}{l}09 / 17 / 97 \\
09 / 17 / 97 \\
09 / 17 / 97 \\
09 / 17 / 97 \\
09 / 17 / 97 \\
09 / 17 / 97 \\
09 / 17 / 97 \\
09 / 17 / 97 \\
09 / 17 / 97 \\
09 / 17 / 97 \\
09 / 17 / 97 \\
09 / 17 / 97 \\
09 / 15 / 97 \\
10 / 23 / 97 \\
09 / 15 / 97 \\
10 / 23 / 97 \\
09 / 15 / 97 \\
10 / 23 / 97 \\
09 / 15 / 97 \\
10 / 23 / 97 \\
09 / 15 / 97 \\
10 / 23 / 97 \\
09 / 15 / 97 \\
10 / 23 / 97 \\
09 / 15 / 97 \\
10 / 23 / 97 \\
09 / 15 / 97 \\
10 / 23 / 97 \\
09 / 15 / 97 \\
10 / 23 / 97 \\
09 / 15 / 97 \\
10 / 23 / 97 \\
09 / 15 / 97 \\
10 / 23 / 97 \\
09 / 15 / 97 \\
10 / 23 / 97 \\
09 / 15 / 97 \\
09 / 15 / 97 \\
09 / 15 / 97 \\
10 / 23 / 97 \\
09 / 15 / 97 \\
10 / 23 / 97 \\
09 / 15 / 97 \\
10 / 23 / 97 \\
09 / 15 / 97 \\
10 / 23 / 97 \\
09 / 15 / 97 \\
09 / 15 / 97 \\
10 / 23 / 97 \\
09 / 15 / 97 \\
10 / 23 / 97 \\
09 / 15 / 97 \\
10 / 23 / 97 \\
09 / 15 / 97 \\
10 / 23 / 97 \\
09 / 15 / 97 \\
10 / 23 / 97 \\
09 / 15 / 97 \\
10 / 23 / 97 \\
09 / 15 / 97 \\
09 / 15 / 97\end{array}$ & 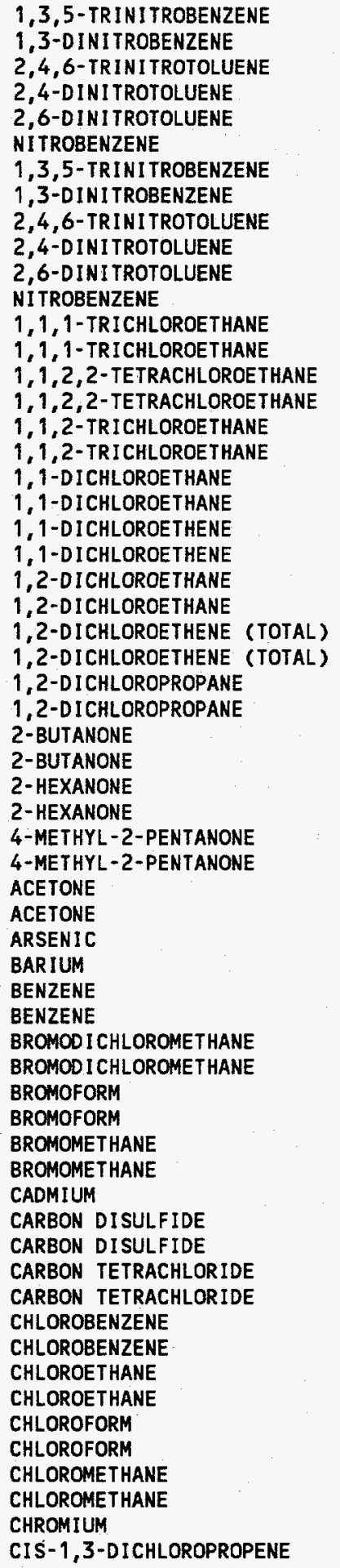 & 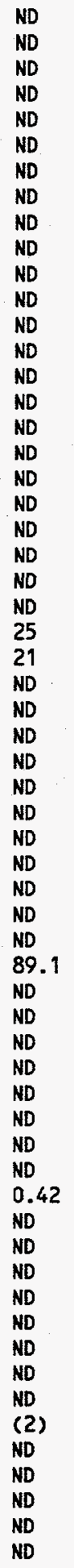 & $\begin{array}{l}0.030 \\
0.090 \\
0.030 \\
0.030 \\
0.010 \\
0.030 \\
0.030 \\
0.090 \\
0.030 \\
0 \\
0.030 \\
0.010 \\
0.030 \\
10 \\
10 \\
10 \\
10 \\
10 \\
10 \\
10 \\
10 \\
10 \\
10 \\
10 \\
10 \\
10 \\
10 \\
10 \\
10 \\
10 \\
10 \\
10 \\
10 \\
10 \\
10 \\
10 \\
10 \\
10 \\
10 \\
10\end{array}$ & 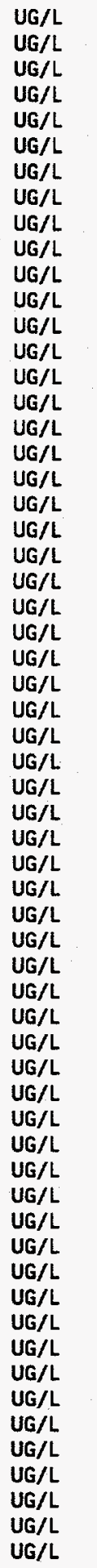 & $\begin{array}{l}\text { USATHAMA } \\
\text { USATHAMA } \\
\text { USATHAMA } \\
\text { USATHAMA } \\
\text { USATHAMA } \\
\text { USATHAMA } \\
\text { USATHAMA } \\
\text { USATHAMA } \\
\text { USATHAMA } \\
\text { USATHAMA } \\
\text { USATHAMA } \\
\text { USATHAMA } \\
\text { EPA CLP } \\
\text { EPA CLP } \\
\text { EPA CLP } \\
\text { EPA CLP } \\
\text { EPA CLP } \\
\text { EPA CLP } \\
\text { EPA CLP } \\
\text { EPA CLP } \\
\text { EPA CLP } \\
\text { EPA CLP } \\
\text { EPA CLP } \\
\text { EPA CLP } \\
\text { EPA CLP } \\
\text { EPA CLP } \\
\text { EPA CLP } \\
\text { EPA CLP } \\
\text { EPA CLP } \\
\text { EPA CLP } \\
\text { EPA CLP } \\
\text { EPA CLP } \\
\text { EPA CLP } \\
\text { EPA CLP } \\
\text { EPA CLP } \\
\text { EPA CLP } \\
\text { EPA 6010A } \\
\text { EPA } 6010 A \\
\text { EPA CLP } \\
\text { EPA CLP } \\
\text { EPA CLP } \\
\text { EPA CLP } \\
\text { EPA CLP } \\
\text { EPA CLP } \\
\text { EPA CLP } \\
\text { EPA CLP } \\
\text { EPA } 6010 A \\
\text { EPA CLP } \\
\text { EPA CLP } \\
\text { EPA CLP } \\
\text { EPA CLP } \\
\text { EPA CLP } \\
\text { EPA CLP } \\
\text { EPA CLP } \\
\text { EPA CLP } \\
\text { EPA CLP } \\
\text { EPA CLP } \\
\text { EPA CLP } \\
\text { EPA CLP } \\
\text { EPA } 6010 A \\
\text { EPA CLP }\end{array}$ \\
\hline
\end{tabular}




\begin{tabular}{|c|c|c|c|c|c|c|}
\hline WSSRAP_ID & DATE_SAM & PARAMETER & CONC & $\mathrm{DL}$ & UNITS & METHOD \\
\hline $\begin{array}{l}\text { GW-2037-1097 } \\
\text { GW-2037-0997 } \\
\text { GW-2037-1097 } \\
\text { GW-2037-0997 } \\
\text { GW-2037-1097 } \\
\text { GW-2037-0997 } \\
\text { GW-2037-0997 } \\
\text { GW-2037-0997 } \\
\text { GW-2037-1097 } \\
\text { GW-2037-0997 } \\
\text { GW-2037-0997 } \\
\text { GW-2037-0997 } \\
\text { GW-2037-0997 } \\
\text { GW-2037-1097 } \\
\text { GW-2037-0997 } \\
\text { GW-2037-0997 } \\
\text { GW-2037-1097 } \\
\text { GW-2037-0997 } \\
\text { GW-2037-1097 } \\
\text { GW-2037-0997 } \\
\text { GW-2037-1097 } \\
\text { GW-2037-0997 } \\
\text { GW-2037-1097 } \\
\text { GW-2037-0997 } \\
\text { GW-2037-1097 } \\
\text { GW-2037-0997 } \\
\text { GW-2037-1097 } \\
\text { GW-2037-0997 } \\
\text { GW-2037-1097 } \\
\text { GW-2038-0997 } \\
\text { GW-2038-1097 } \\
\text { GW-2038-0997 } \\
\text { GW-2038-1097 } \\
\text { GW-2038-0997 } \\
\text { GW-2038-1097 } \\
\text { GW-2038-0997 } \\
\text { GW-2038-1097 } \\
\text { GW-2038-0997 } \\
\text { GW-2038-1097 } \\
\text { GW-2038-0997 } \\
\text { GW-2038-1097 } \\
\text { GW-2038-0997 } \\
\text { GW-2038-1097 } \\
\text { GW-2038-0997 } \\
\text { GW-2038-1097 } \\
\text { GW-2038-0997 } \\
\text { GW-2038-1097 } \\
\text { GW-2038-0997 } \\
\text { GW-2038-1097 } \\
\text { GW-2038-0997 } \\
\text { GW-2038-1097 } \\
\text { GW-2038-0997-2038-0997-0997 } \\
\text { GW-2038-1097 } \\
\text { GW-2038-0897 } \\
\text { GW-2038-0997 } \\
\text { GW-2038-0997 }\end{array}$ & $\begin{array}{l}10 / 23 / 97 \\
09 / 15 / 97 \\
10 / 23 / 97 \\
09 / 15 / 97 \\
10 / 23 / 97 \\
09 / 15 / 97 \\
09 / 15 / 97 \\
09 / 15 / 97 \\
10 / 23 / 97 \\
09 / 15 / 97 \\
09 / 15 / 97 \\
09 / 15 / 97 \\
09 / 15 / 97 \\
10 / 23 / 97 \\
09 / 15 / 97 \\
09 / 15 / 97 \\
10 / 23 / 97 \\
09 / 15 / 97 \\
10 / 23 / 97 \\
09 / 15 / 97 \\
10 / 23 / 97 \\
09 / 15 / 97 \\
10 / 23 / 97 \\
09 / 15 / 97 \\
10 / 23 / 97 \\
09 / 15 / 97 \\
10 / 23 / 97 \\
09 / 15 / 97 \\
10 / 23 / 97 \\
09 / 15 / 97 \\
10 / 23 / 97 \\
09 / 15 / 97 \\
10 / 23 / 97 \\
09 / 15 / 97 \\
10 / 23 / 97 \\
09 / 15 / 97 \\
10 / 23 / 97 \\
09 / 15 / 97 \\
10 / 23 / 97 \\
09 / 15 / 97 \\
10 / 23 / 97 \\
09 / 15 / 97 \\
10 / 23 / 97 \\
09 / 15 / 97 \\
10 / 23 / 97 \\
09 / 15 / 97 \\
10 / 23 / 97 \\
09 / 15 / 97 \\
10 / 23 / 97 \\
09 / 15 / 97 \\
10 / 23 / 97 \\
09 / 15 / 97 \\
10 / 23 / 97 \\
08 / 14 / 97 \\
09 / 15 / 97 \\
08 / 14 / 97 \\
09 / 15 / 97 \\
09 / 15 / 97 \\
10 / 23 / 97 \\
09 / 15 / 97 \\
10 / 23 / 97\end{array}$ & 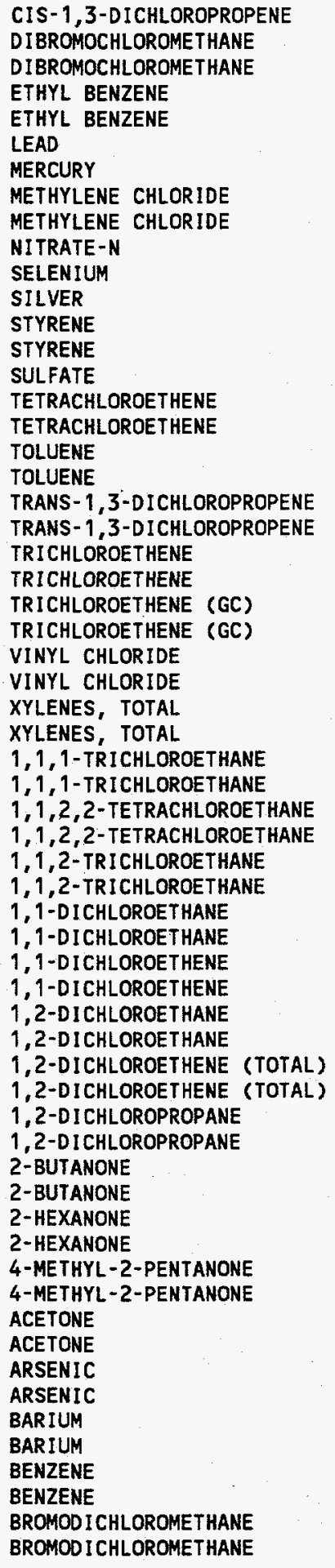 & $\begin{array}{l}\text { ND } \\
\text { ND } \\
\text { ND } \\
\text { ND } \\
\text { ND } \\
\text { ND } \\
3.9 \\
\text { ND } \\
\text { ND } \\
248 \\
5.7 \\
1.8 \\
\text { ND } \\
\text { ND } \\
140 \\
\text { (1) } \\
\text { ND } \\
\text { ND } \\
\text { ND } \\
\text { ND } \\
\text { ND } \\
1100 \\
370 \\
1200 \\
1280 \\
\text { ND } \\
\text { ND } \\
\text { ND } \\
\text { ND } \\
\text { ND } \\
\text { ND } \\
\text { ND } \\
\text { ND } \\
\text { ND } \\
\text { ND } \\
\text { ND } \\
\text { ND } \\
\text { ND } \\
\text { ND } \\
\text { ND } \\
\text { ND } \\
19 \\
13 \\
\text { ND } \\
\text { ND } \\
\text { ND } \\
\text { ND } \\
\text { ND } \\
\text { ND } \\
\text { ND } \\
\text { ND } \\
150 \\
\text { ND } \\
\text { ND } \\
\text { ND } \\
231 \\
\text { ND } \\
\text { ND } \\
\text { ND } \\
\text { ND }\end{array}$ & 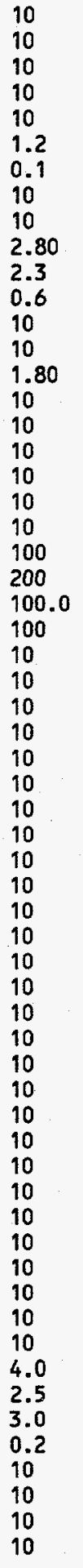 & $\begin{array}{l}U G / L \\
U G / L \\
U G / L \\
U G / L \\
U G / L \\
U G / L \\
U G / L \\
U G / L \\
U G / L \\
M G / L \\
U G / L \\
U G / L \\
U G / L \\
U G / L \\
U G / L \\
U G / L \\
U G / L \\
U G / L \\
U G / L \\
U G / L \\
U G / L \\
U G / L \\
U G / L \\
U G / L \\
U G / L \\
U G / L \\
U G / L \\
U G / L \\
U G / L \\
U G / L \\
U G / L \\
U G / L \\
U G / L \\
U G / L \\
U G / L \\
U G / L \\
U G / L \\
U G / L \\
U G / L \\
U G / L \\
U G / L \\
U G / L \\
U G / L \\
U G / L \\
U G / L \\
U G / L \\
U G / L \\
U G / L \\
U G / L \\
U G / L \\
U G / L \\
U G / L \\
U G / L \\
U G / L \\
U G / L \\
U G / L \\
U G / L \\
U\end{array}$ & $\begin{array}{l}\text { EPA CLP } \\
\text { EPA CLP } \\
\text { EPA CLP } \\
\text { EPA CLP } \\
\text { EPA CLP } \\
\text { EPA } 6010 A \\
\text { EPA7470A } \\
\text { EPA CLP } \\
\text { EPA CLP } \\
\text { EPA } 353.1 \\
\text { EPA } 6010 A \\
\text { EPA } 6010 A \\
\text { EPA CLP } \\
\text { EPA CLP } \\
\text { EPA } 300.0 \\
\text { EPA CLP } \\
\text { EPA CLP } \\
\text { EPA CLP } \\
\text { EPA CLP } \\
\text { EPA CLP } \\
\text { EPA CLP } \\
\text { EPA CLP } \\
\text { EPA CLP } \\
\text { EPA } 601 \\
\text { EPA } 601 \\
\text { EPA CLP } \\
\text { EPA CLP } \\
\text { EPA CLP } \\
\text { EPA CLP } \\
\text { EPA CLP } \\
\text { EPA CLP } \\
\text { EPA CLP } \\
\text { EPA CLP } \\
\text { EPA CLP } \\
\text { EPA CLP } \\
\text { EPA CLP } \\
\text { EPA CLP } \\
\text { EPA CLP } \\
\text { EPA CLP } \\
\text { EPA CLP } \\
\text { EPA CLP } \\
\text { EPA CLP } \\
\text { EPA CLP } \\
\text { EPA CLP } \\
\text { EPA CLP } \\
\text { EPA CLP } \\
\text { EPA } C L P \\
\text { EPA CLP } \\
\text { EPA } \text { CLP } \\
\text { EPA CLP } \\
\text { EPA CLP } \\
\text { EPA CLP } \\
\text { EPA } C L P \\
\text { EPA CLP } \\
\text { EPA } 6010 A \\
\text { EPA CLP } \\
\text { EPA } 6010 A \\
\text { EPA CLP } \\
\text { EPA CLP } \\
\text { EPA CLP } \\
\text { EPA } \text { CLP }\end{array}$ \\
\hline
\end{tabular}




\begin{tabular}{|c|c|c|c|c|c|c|c|}
\hline WSSRAP_ID & DATE_SAM & PARAMETER & CONC & $D L$ & UNITS & METHOD & \\
\hline 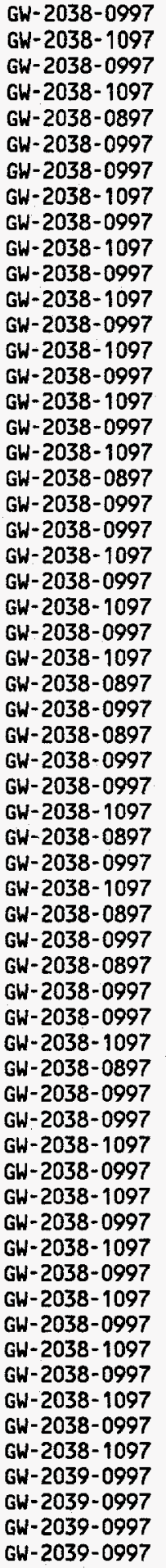 & $\begin{array}{l}09 / 15 / 97 \\
10 / 23 / 97 \\
09 / 15 / 97 \\
10 / 23 / 97 \\
08 / 14 / 97 \\
09 / 15 / 97 \\
09 / 15 / 97 \\
10 / 23 / 97 \\
09 / 15 / 97 \\
10 / 23 / 97 \\
09 / 15 / 97 \\
10 / 23 / 97 \\
09 / 15 / 97 \\
10 / 23 / 97 \\
09 / 15 / 97 \\
10 / 23 / 97 \\
09 / 15 / 97 \\
10 / 23 / 97 \\
08 / 14 / 97 \\
09 / 15 / 97 \\
09 / 15 / 97 \\
10 / 23 / 97 \\
09 / 15 / 97 \\
10 / 23 / 97 \\
09 / 15 / 97 \\
10 / 23 / 97 \\
08 / 14 / 97 \\
09 / 15 / 97 \\
08 / 14 / 97 \\
09 / 15 / 97 \\
09 / 15 / 97 \\
10 / 23 / 97 \\
08 / 14 / 97 \\
09 / 15 / 97 \\
10 / 23 / 97 \\
08 / 14 / 97 \\
09 / 15 / 97 \\
08 / 14 / 97 \\
09 / 15 / 97 \\
09 / 15 / 97 \\
10 / 23 / 97 \\
08 / 14 / 97 \\
09 / 15 / 97 \\
09 / 15 / 97 \\
10 / 23 / 97 \\
09 / 15 / 97 \\
10 / 23 / 97 \\
09 / 15 / 97 \\
10 / 23 / 97 \\
09 / 15 / 97 \\
10 / 23 / 97 \\
09 / 15 / 97 \\
10 / 23 / 97 \\
09 / 15 / 97 \\
10 / 23 / 97 \\
09 / 15 / 97 \\
10 / 23 / 97 \\
09 / 15 / 97 \\
09 / 15 / 97 \\
09 / 15 / 97 \\
09 / 15 / 97\end{array}$ & 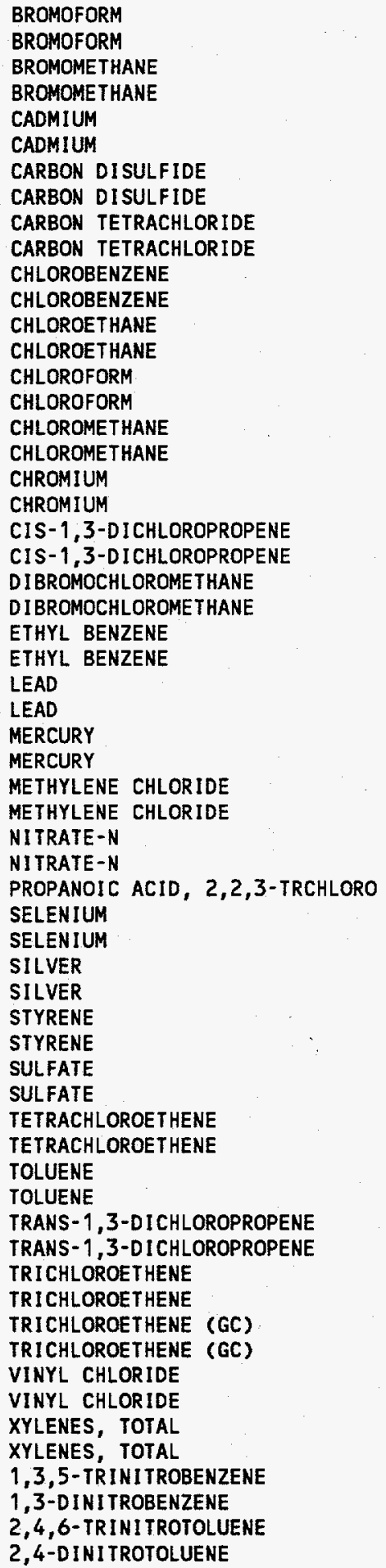 & 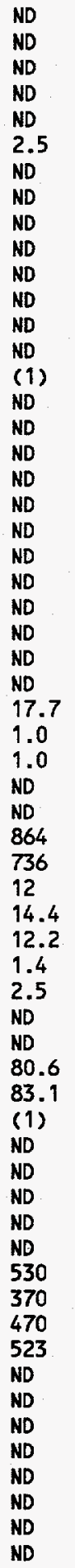 & $\begin{array}{l}10 \\
10 \\
10 \\
10 \\
10 \\
3 \\
3.0 \\
0.3 \\
10 \\
10 \\
10 \\
10 \\
10 \\
10 \\
10 \\
10 \\
10 \\
10 \\
10 \\
10 \\
10 \\
10 \\
10 \\
10 \\
10 \\
4.0 \\
0\end{array}$ & 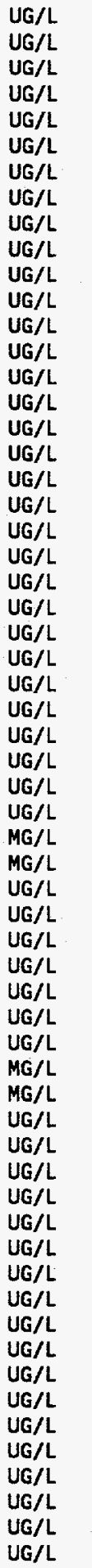 & $\begin{array}{l}\text { EPA CLP } \\
\text { EPA CLP } \\
\text { EPA CLP } \\
\text { EPA CLP } \\
\text { EPA CLP } \\
\text { EPA } 6010 A \\
\text { EPA CLP } \\
\text { EPA CLP } \\
\text { EPA CLP } \\
\text { EPA CLP } \\
\text { EPA CLP } \\
\text { EPA CLP } \\
\text { EPA CLP } \\
\text { EPA CLP } \\
\text { EPA CLP } \\
\text { EPA CLP } \\
\text { EPA CLP } \\
\text { EPA CLP } \\
\text { EPA CLP } \\
\text { EPA } 6010 A \\
\text { EPA CLP } \\
\text { EPA CLP } \\
\text { EPA CLP } \\
\text { EPA CLP } \\
\text { EPA CLP } \\
\text { EPA CLP } \\
\text { EPA CLP } \\
\text { EPA } 6010 A \\
\text { EPA CLP } \\
\text { EPA7470A } \\
\text { EPA CLP } \\
\text { EPA CLP } \\
\text { EPA } 353.1 \\
\text { EPA } 353.1 \\
\text { EPA CLP } \\
\text { EPA CLP } \\
\text { EPA } 6010 A \\
\text { EPA CLP } \\
\text { EPA } 6010 A \\
\text { EPA CLP } \\
\text { EPA CLP } \\
\text { EPA } 300.0 \\
\text { EPA } 300.0 \\
\text { EPA CLP } \\
\text { EPA CLP } \\
\text { EPA CLP } \\
\text { EPA CLP } \\
\text { EPA CLP } \\
\text { EPA CLP } \\
\text { EPA CLP } \\
\text { EPA CLP } \\
\text { EPA } 601 \\
\text { EPA } 601 \\
\text { EPA CLP } \\
\text { EPA CLP } \\
\text { EPA CLP } \\
\text { EPA CLP } \\
\text { USATHAMA } \\
\text { USATHAMA } \\
\text { USATHAMA } \\
\text { USATHAMA }\end{array}$ & , \\
\hline
\end{tabular}




\begin{tabular}{|c|c|c|c|c|c|c|}
\hline WSSRAP_ID & DATE_SAM & PARAMETER & CONC & $\mathrm{DL}$ & UNITS & METHOD \\
\hline $\begin{array}{l}\text { GW-2039-0997 } \\
\text { GW-2039-0897 } \\
\text { GW-2039-0997 } \\
\text { GW-2039-0897 } \\
\text { GW-2039-0997 } \\
\text { GW-2039-0897 } \\
\text { GW-2039-0997 } \\
\text { GW-2039-0997 } \\
\text { GW-2039-0897 } \\
\text { GW-2039-0997 } \\
\text { GW-2039-0997 } \\
\text { GW-2039-0897 } \\
\text { GW-2039-0997 } \\
\text { GW-2039-0897 } \\
\text { GW-2039-0997 } \\
\text { GW-2039-0897 } \\
\text { GW-2039-0997 } \\
\text { GW-2039-0997 } \\
\text { GW-2039-0897 } \\
\text { GW-2039-0997 } \\
\text { GW-2039-0897 } \\
\text { GW-2039-0997 } \\
\text { GW-2039-0897 } \\
\text { GW-2039-0997 } \\
\text { GW-2039-0997 } \\
\text { GW-2040-0397 } \\
\text { GW-2040-0397 } \\
\text { GW-2040-0397 } \\
\text { GW-2040-0397 } \\
\text { GW-2040-0397 } \\
\text { GW-2040-Q397 } \\
\text { GW-2041-0397 } \\
\text { GW-2041-Q397 } \\
\text { GW-2041-Q397 } \\
\text { GW-2041-0397 } \\
\text { GW-2041-0397 } \\
\text { GW-2041-Q397 } \\
\text { GW-2042-Q397 } \\
\text { GW-2042-0397 } \\
\text { GW-2042-0397 } \\
\text { GW-2042-0397 } \\
\text { GW-2042-Q397 } \\
\text { GW-2042-0397 } \\
\text { GW-2043-0397 } \\
\text { GW-2043-0397 } \\
\text { GW-2043-03997 } \\
\text { GW-2043-0397 } \\
\text { GW-2043-0397 } \\
\text { GW-2043-0397 } \\
\text { GW-2044-A197 } \\
\text { GW-2044-A197 } \\
\text { GW-2044-A197 } \\
\text { GW-2044-A197 } \\
\text { GW-2044-A197 } \\
\text { GW-2044-A197 } \\
\text { GW-2044-A197 } \\
\text { GW-2044-A197 } \\
\text { GW-2044-A197 } \\
\text { GW-2044-A197 }\end{array}$ & $\begin{array}{l}09 / 15 / 97 \\
08 / 14 / 97 \\
09 / 15 / 97 \\
08 / 14 / 97 \\
09 / 15 / 97 \\
08 / 14 / 97 \\
09 / 15 / 97 \\
09 / 15 / 97 \\
08 / 14 / 97 \\
09 / 15 / 97 \\
09 / 15 / 97 \\
08 / 14 / 97 \\
09 / 15 / 97 \\
08 / 14 / 97 \\
09 / 15 / 97 \\
08 / 14 / 97 \\
09 / 15 / 97 \\
09 / 15 / 97 \\
08 / 14 / 97 \\
09 / 15 / 97 \\
08 / 14 / 97 \\
09 / 15 / 97 \\
08 / 14 / 97 \\
09 / 15 / 97 \\
09 / 15 / 97 \\
09 / 17 / 97 \\
09 / 17 / 97 \\
09 / 17 / 97 \\
09 / 17 / 97 \\
09 / 17 / 97 \\
09 / 17 / 97 \\
09 / 17 / 97 \\
09 / 17 / 97 \\
09 / 17 / 97 \\
09 / 17 / 97 \\
09 / 17 / 97 \\
09 / 17 / 97 \\
09 / 22 / 97 \\
09 / 22 / 97 \\
09 / 22 / 97 \\
09 / 22 / 97 \\
09 / 22 / 97 \\
09 / 22 / 97 \\
09 / 22 / 97 \\
09 / 22 / 97 \\
09 / 22 / 97 \\
09 / 22 / 97 \\
09 / 22 / 97 \\
09 / 22 / 97 \\
08 / 27 / 97 \\
08 / 27 / 97 \\
08 / 27 / 97 \\
08 / 27 / 97 \\
08 / 27 / 97 \\
08 / 27 / 97 \\
08 / 27 / 97 \\
08 / 27 / 97 \\
08 / 27 / 97 \\
08 / 27 / 97 \\
08 / 27 / 97 \\
08 / 27 / 97\end{array}$ & 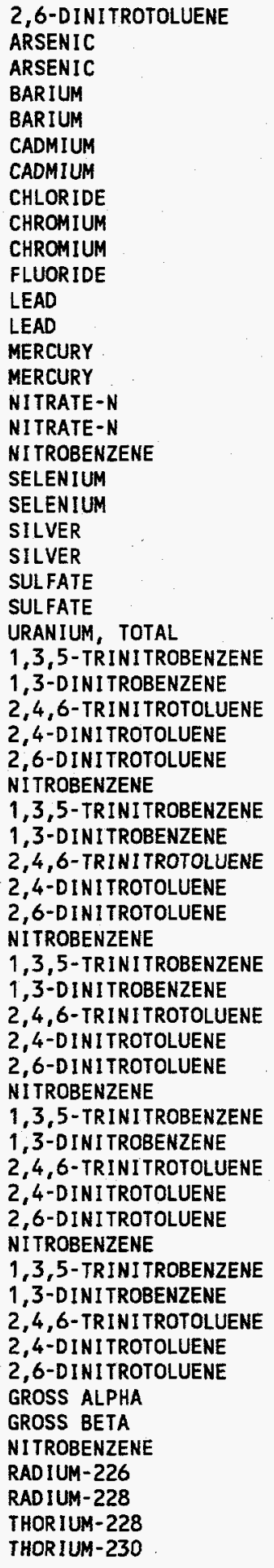 & $\begin{array}{l}\text { ND } \\
\text { ND } \\
107 \\
269 \\
319 \\
\text { ND } \\
0.34 \\
52.1 \\
4.6 \\
3.3 \\
\text { ND } \\
\text { ND } \\
2.2 \\
\text { ND } \\
\text { ND } \\
47.4 \\
58.4 \\
\text { ND } \\
\text { ND } \\
6.1 \\
2.8 \\
1.5 \\
31.7 \\
28.5 \\
2.83 \\
\text { ND } \\
\text { ND } \\
\text { ND } \\
\text { CO.01 } \\
\text { ND } \\
\text { ND } \\
\text { ND } \\
\text { ND } \\
\text { ND } \\
\text { ND } \\
\text { ND } \\
\text { ND } \\
\text { ND } \\
\text { ND } \\
\text { ND } \\
\text { ND } \\
\text { ND } \\
\text { ND } \\
\text { ND } \\
\text { ND } \\
\text { ND } \\
0.079 \\
\text { ND } \\
\text { ND } \\
\text { ND } \\
\text { ND } \\
\text { ND } \\
\text { ND } \\
\text { ND } \\
23.2 \\
11.8 \\
\text { ND } \\
\text { CO.18 } \\
2.76 \\
\text { CO.02 } \\
\text { O.00 }\end{array}$ & 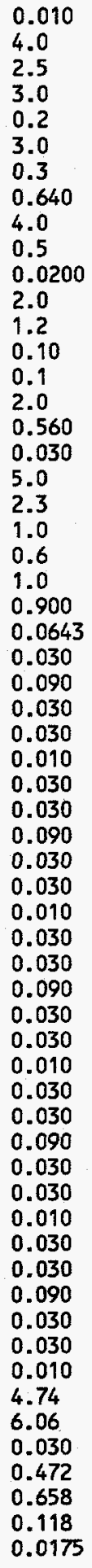 & 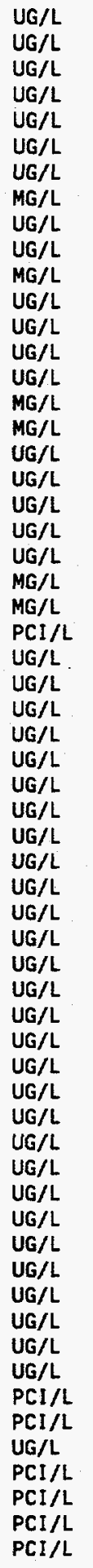 & $\begin{array}{l}\text { USATHAMA } \\
\text { EPA CLP } \\
\text { EPA 6010A } \\
\text { EPA CLP } \\
\text { EPA 6010A } \\
\text { EPA CLP } \\
\text { EPA 601CA } \\
\text { EPA } 300.0 \\
\text { EPA CLP } \\
\text { EPA 6010A } \\
\text { EPA 300.0 } \\
\text { EPA CLP } \\
\text { EPA 6010A } \\
\text { EPA CLP } \\
\text { EPA7470A } \\
\text { EPA 353.1 } \\
\text { EPA } 353.1 \\
\text { USATHAMA } \\
\text { EPA CLP } \\
\text { EPA 6010A } \\
\text { EPA CLP } \\
\text { EPA 6010A } \\
\text { EPA 300.0 } \\
\text { EPA 300.0 } \\
\text { ASTM 5174-91 } \\
\text { USATHAMA } \\
\text { USATHAMA } \\
\text { USATHAMA } \\
\text { USATHAMA } \\
\text { USATHAMA } \\
\text { USATHAMA } \\
\text { USATHAMA } \\
\text { USATHAMA } \\
\text { USATHAMA } \\
\text { USATHAMA } \\
\text { USATHAMA } \\
\text { USATHAMA } \\
\text { USATHAMA } \\
\text { USATHAMA } \\
\text { USATHAMA } \\
\text { USATHAMA } \\
\text { USATHAMA } \\
\text { USATHAMA } \\
\text { USATHAMA } \\
\text { USATHAMA } \\
\text { USATHAMA } \\
\text { USATHAMA } \\
\text { USATHAMA } \\
\text { USATHAMA } \\
\text { USATHAMA } \\
\text { USATHAMA } \\
\text { USATHAMA } \\
\text { USATHAMA } \\
\text { USATHAMA } \\
\text { EPA 900.0 } \\
\text { EPA 900.0 } \\
\text { USATHAMA } \\
\text { EPA 903.1 } \\
\text { EPA 904.0 } \\
\text { HASL } 300 \\
\text { HASL 300 } \\
\text { HSA }\end{array}$ \\
\hline
\end{tabular}




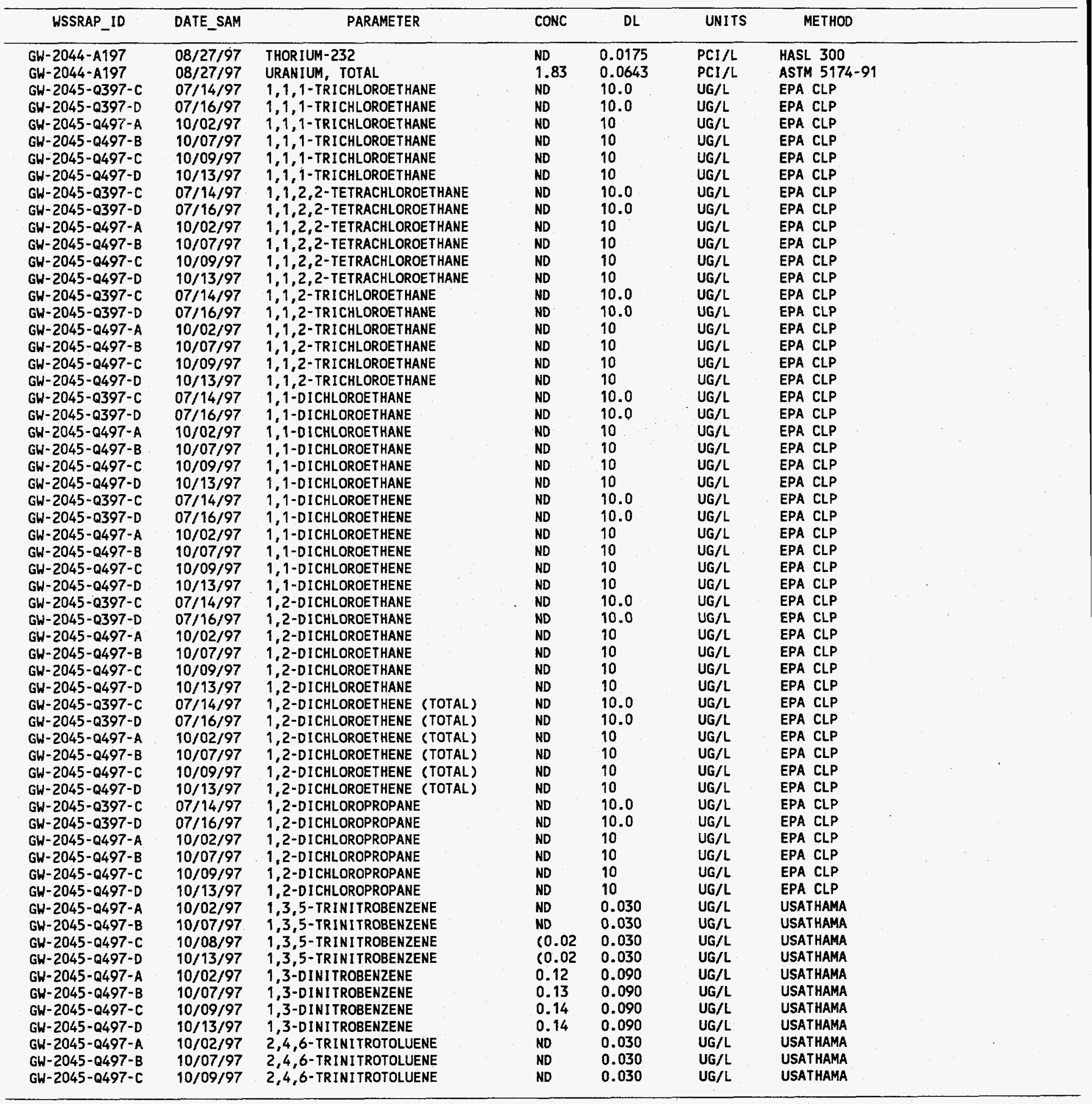




\begin{tabular}{|c|c|c|c|c|c|c|}
\hline WSSRAP_ID & DATE_SAM & PARAMETER & CONC & $D L$ & UNITS & METHOD \\
\hline 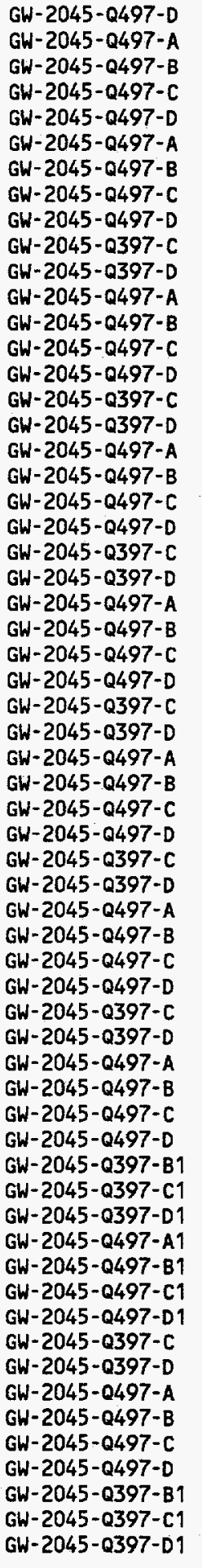 & $\begin{array}{l}10 / 13 / 97 \\
10 / 02 / 97 \\
10 / 07 / 97 \\
10 / 09 / 97 \\
10 / 13 / 97 \\
10 / 02 / 97 \\
10 / 07 / 97 \\
10 / 09 / 97 \\
10 / 13 / 97 \\
07 / 14 / 97 \\
07 / 16 / 97 \\
10 / 02 / 97 \\
10 / 07 / 97 \\
10 / 09 / 97 \\
10 / 13 / 97 \\
07 / 14 / 97 \\
07 / 16 / 97 \\
10 / 02 / 97 \\
10 / 07 / 97 \\
10 / 09 / 97 \\
10 / 13 / 97 \\
07 / 14 / 97 \\
07 / 16 / 97 \\
10 / 02 / 97 \\
10 / 07 / 97 \\
10 / 09 / 97 \\
10 / 13 / 97 \\
07 / 14 / 97 \\
07 / 16 / 97 \\
10 / 02 / 97 \\
10 / 07 / 97 \\
10 / 09 / 97 \\
10 / 13 / 97 \\
07 / 14 / 97 \\
07 / 16 / 97 \\
10 / 02 / 97 \\
10 / 07 / 97 \\
10 / 09 / 97 \\
10 / 13 / 97 \\
07 / 14 / 97 \\
07 / 16 / 97 \\
10 / 02 / 97 \\
10 / 07 / 97 \\
10 / 09 / 97 \\
10 / 13 / 97 \\
07 / 11 / 97 \\
07 / 15 / 97 \\
07 / 17 / 97 \\
10 / 03 / 97 \\
10 / 08 / 97 \\
10 / 10 / 97 \\
10 / 14 / 97 \\
07 / 14 / 97 \\
07 / 16 / 97 \\
10 / 02 / 97 \\
10 / 07 / 97 \\
10 / 09 / 97 \\
10 / 13 / 97 \\
07 / 11 / 97 \\
07 / 15 / 97 \\
07 / 17 / 97\end{array}$ & 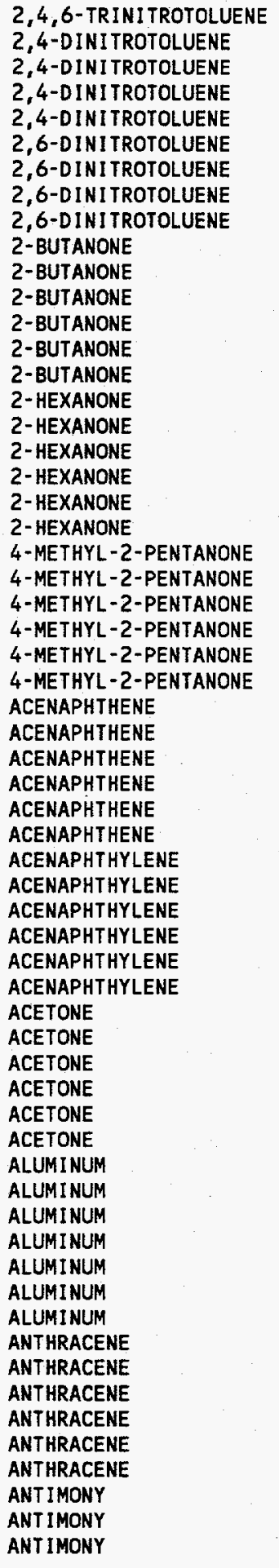 & 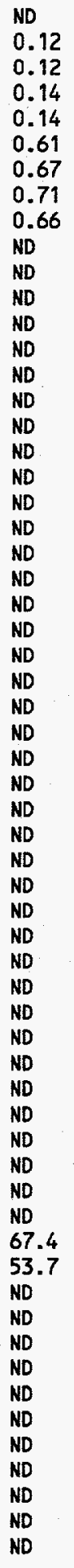 & 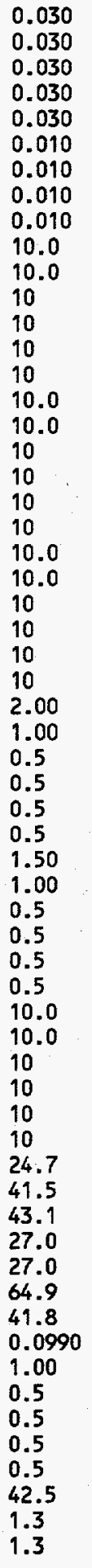 & $\begin{array}{l}\text { UG/L } \\
U G / L \\
U G / L \\
U G / L \\
U G / L \\
U G / L \\
U G / L \\
U G / L \\
U G / L \\
U G / L \\
U G / L \\
U G / L \\
U G / L \\
U G / L \\
U G / L \\
U G / L \\
U G / L \\
U G / L \\
U G / L \\
U G / L \\
U G / L \\
U G / L \\
U G / L \\
U G / L \\
U G / L \\
U G / L \\
U G / L \\
U G / L \\
U G / L \\
U G / L \\
U G / L \\
U G / L \\
U G / L \\
U G / L \\
U G / L \\
U G / L \\
U G / L \\
U G / L \\
U G / L \\
U G / L \\
U G / L \\
U G / L \\
U G / L \\
U G / L \\
U G / L \\
U G / L \\
U G / L \\
U G / L \\
U G / L \\
U G / L \\
U G / L \\
U G / L \\
U G / L \\
U G / L \\
U G / L \\
U G / L \\
U G / L \\
U G / L \\
U\end{array}$ & $\begin{array}{l}\text { USATHAMA } \\
\text { USATHAMA } \\
\text { USATHAMA } \\
\text { USATHAMA } \\
\text { USATHAMA } \\
\text { USATHAMA } \\
\text { USATHAMA } \\
\text { USATHAMA } \\
\text { USATHAMA } \\
\text { EPA CLP } \\
\text { EPA CLP } \\
\text { EPA CLP } \\
\text { EPA CLP } \\
\text { EPA CLP } \\
\text { EPA CLP } \\
\text { EPA CLP } \\
\text { EPA CLP } \\
\text { EPA CLP } \\
\text { EPA CLP } \\
\text { EPA CLP } \\
\text { EPA CLP } \\
\text { EPA CLP } \\
\text { EPA CLP } \\
\text { EPA CLP } \\
\text { EPA CLP } \\
\text { EPA CLP } \\
\text { EPA CLP } \\
\text { EPA } 8310 \\
\text { EPA } 8270 A \\
\text { EPA } 8310 \\
\text { EPA } 8310 \\
\text { SH846 } 8310 \\
\text { EPA } 8310 \\
\text { EPA } 8310 \\
\text { EPA } 8270 A \\
\text { EPA } 8310 \\
\text { EPA } 8310 \\
\text { SW846 } 8310 \\
\text { EPA } 8310 \\
\text { EPA CLP } \\
\text { EPA CLP } \\
\text { EPA CLP } \\
\text { EPA CLP } \\
\text { EPA CLP } \\
\text { EPA CLP } \\
\text { EPA CLP } \\
\text { EPA } 6010 A \\
\text { EPA } 6010 A \\
\text { EPA } 6010 A \\
\text { EPA } 6010 A \\
\text { EPA } 6010 A \\
\text { EPA } 6010 A \\
\text { EPA } 8310 \\
\text { EPA } 8270 A \\
\text { EPA } 8310 \\
\text { EPA } 8310 \\
\text { SWB46 } 8310 \\
\text { EPA } 8310 \\
\text { EPA CLP } \\
\text { EPA } 6010 A \\
\text { EPA } 6010 A\end{array}$ \\
\hline
\end{tabular}




\begin{tabular}{|c|c|c|c|c|c|c|}
\hline WSSRAP_ID & DATE_SAM & PARAMETER & CONC & $D L$ & UNITS & METHOD \\
\hline 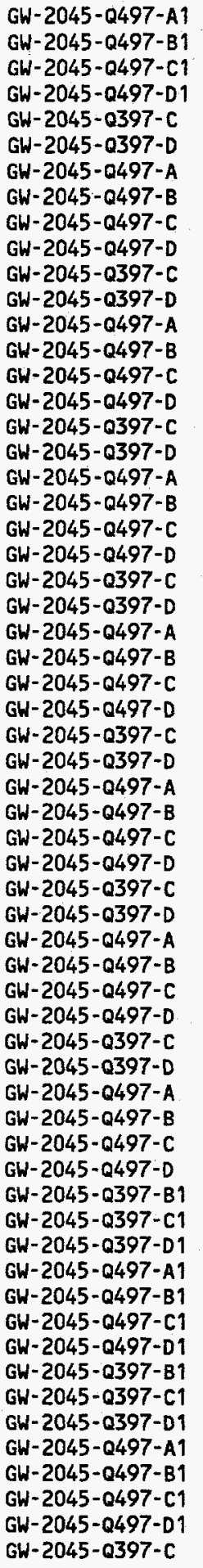 & $\begin{array}{l}10 / 03 / 97 \\
10 / 08 / 97 \\
10 / 10 / 97 \\
10 / 14 / 97 \\
07 / 14 / 97 \\
07 / 16 / 97 \\
10 / 02 / 97 \\
10 / 07 / 97 \\
10 / 09 / 97 \\
10 / 13 / 97 \\
07 / 14 / 97 \\
07 / 16 / 97 \\
10 / 02 / 97 \\
10 / 07 / 97 \\
10 / 09 / 97 \\
10 / 13 / 97 \\
07 / 14 / 97 \\
07 / 16 / 97 \\
10 / 02 / 97 \\
10 / 07 / 97 \\
10 / 09 / 97 \\
10 / 13 / 97 \\
07 / 14 / 97 \\
07 / 16 / 97 \\
10 / 02 / 97 \\
10 / 07 / 97 \\
10 / 09 / 97 \\
10 / 13 / 97 \\
07 / 14 / 97 \\
07 / 16 / 97 \\
10 / 02 / 97 \\
10 / 07 / 97 \\
10 / 09 / 97 \\
10 / 13 / 97 \\
07 / 14 / 97 \\
07 / 16 / 97 \\
10 / 02 / 97 \\
10 / 07 / 97 \\
10 / 09 / 97 \\
10 / 13 / 97 \\
07 / 14 / 97 \\
07 / 16 / 97 \\
10 / 02 / 97 \\
10 / 07 / 97 \\
10 / 09 / 97 \\
10 / 13 / 97 \\
07 / 11 / 97 \\
07 / 15 / 97 \\
07 / 17 / 97 \\
10 / 03 / 97 \\
10 / 08 / 97 \\
10 / 10 / 97 \\
10 / 14 / 97 \\
07 / 11 / 97 \\
07 / 15 / 97 \\
07 / 17 / 97 \\
10 / 03 / 97 \\
10 / 08 / 97 \\
10 / 10 / 97 \\
10 / 14 / 97 \\
07 / 14 / 97\end{array}$ & 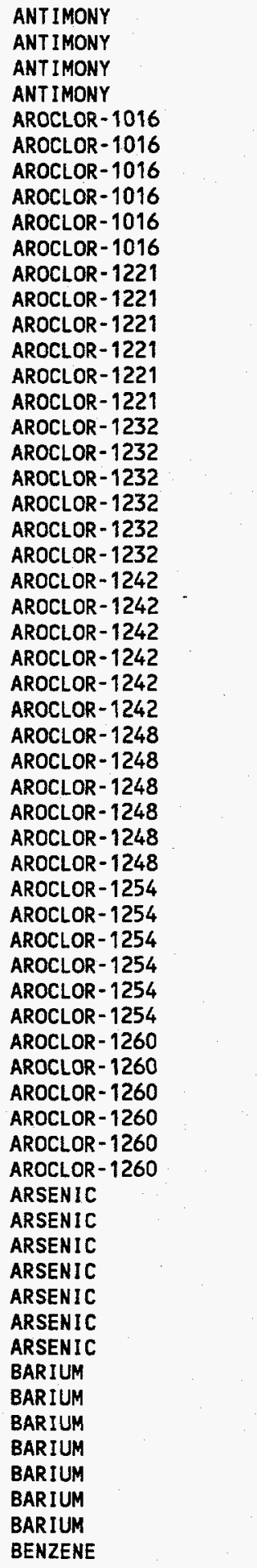 & $\begin{array}{l}\text { ND } \\
\text { ND } \\
\text { ND } \\
\text { ND } \\
\text { ND } \\
\text { ND } \\
\text { ND } \\
\text { ND } \\
\text { ND } \\
\text { ND } \\
\text { ND } \\
\text { ND } \\
\text { ND } \\
\text { ND } \\
\text { ND } \\
\text { ND } \\
\text { ND } \\
\text { ND } \\
\text { ND } \\
\text { ND } \\
\text { ND } \\
\text { ND } \\
\text { ND } \\
\text { ND } \\
\text { ND } \\
\text { ND } \\
\text { ND } \\
\text { ND } \\
\text { ND } \\
\text { ND } \\
\text { ND } \\
\text { ND } \\
\text { ND } \\
\text { ND } \\
\text { ND } \\
\text { ND } \\
\text { ND } \\
\text { ND } \\
\text { ND } \\
\text { ND } \\
\text { ND } \\
\text { ND } \\
\text { ND } \\
\text { ND } \\
\text { ND } \\
\text { ND } \\
\text { ND } \\
\text { ND } \\
\text { ND } \\
\text { ND } \\
\text { ND } \\
\text { ND } \\
\text { ND } \\
\text { 185 } \\
185 \\
187 \\
214 \\
209 \\
208 \\
202 \\
\text { ND }\end{array}$ & $\begin{array}{l}11.0 \\
11.0 \\
11.0 \\
11.0 \\
1.00 \\
0.12 \\
1.0 \\
1.0 \\
1.0 \\
1.0 \\
2.00 \\
0.12 \\
1.0 \\
1.0 \\
1.0 \\
1.0 \\
1.00 \\
0.12 \\
1.0 \\
1.0 \\
1.0 \\
1.0 \\
1.00 \\
0.12 \\
1.0 \\
1.0 \\
1.0 \\
1.0 \\
1.00 \\
0.12 \\
1.0 \\
1.0 \\
1.0 \\
1.0 \\
1.00 \\
0.12 \\
1.0 \\
1.0 \\
1.0 \\
1.0 \\
1.00 \\
0.12 \\
1.0 \\
1.0 \\
1.0 \\
1.0 \\
1.7 \\
1.7 \\
1.7 \\
4.0 \\
4.0 \\
4.0 \\
4.0 \\
0.60 \\
0.30 \\
0.30 \\
2.0 \\
2.0 \\
2.0 \\
2.0 \\
10.0\end{array}$ & 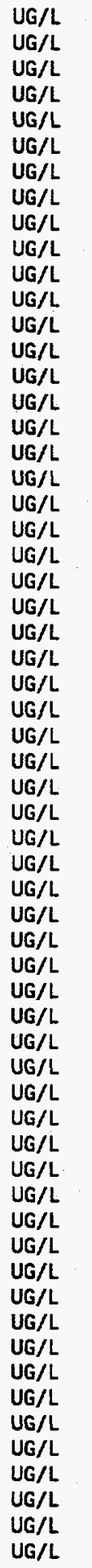 & 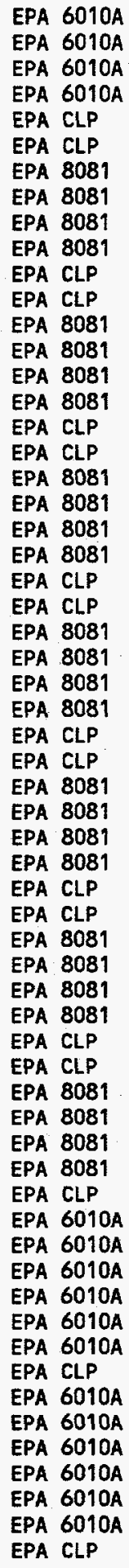 \\
\hline
\end{tabular}




\begin{tabular}{|c|c|c|c|c|c|c|}
\hline WSSRAP_ID & DATE_SAM & PARAMETER & CONC & $D L$ & UNITS & METHOD \\
\hline 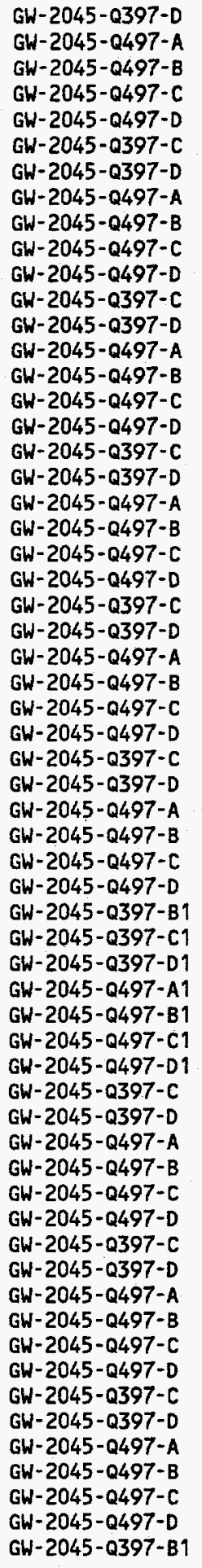 & $\begin{array}{l}07 / 16 / 97 \\
10 / 02 / 97 \\
10 / 07 / 97 \\
10 / 09 / 97 \\
10 / 13 / 97 \\
07 / 14 / 97 \\
07 / 16 / 97 \\
10 / 02 / 97 \\
10 / 07 / 97 \\
10 / 09 / 97 \\
10 / 13 / 97 \\
07 / 14 / 97 \\
07 / 16 / 97 \\
10 / 02 / 97 \\
10 / 07 / 97 \\
10 / 09 / 97 \\
10 / 13 / 97 \\
07 / 14 / 97 \\
07 / 16 / 97 \\
10 / 02 / 97 \\
10 / 07 / 97 \\
10 / 09 / 97 \\
10 / 13 / 97 \\
07 / 14 / 97 \\
07 / 16 / 97 \\
10 / 02 / 97 \\
10 / 07 / 97 \\
10 / 09 / 97 \\
10 / 13 / 97 \\
07 / 14 / 97 \\
07 / 16 / 97 \\
10 / 02 / 97 \\
10 / 07 / 97 \\
10 / 09 / 97 \\
10 / 13 / 97 \\
07 / 11 / 97 \\
07 / 15 / 97 \\
07 / 17 / 97 \\
10 / 03 / 97 \\
10 / 08 / 97 \\
10 / 10 / 97 \\
10 / 14 / 97 \\
07 / 14 / 97 \\
07 / 16 / 97 \\
10 / 02 / 97 \\
10 / 07 / 97 \\
10 / 09 / 97 \\
10 / 13 / 97 \\
07 / 14 / 97 \\
07 / 16 / 97 \\
10 / 02 / 97 \\
10 / 07 / 97 \\
10 / 09 / 97 \\
10 / 13 / 97 \\
07 / 14 / 97 \\
07 / 16 / 97 \\
10 / 02 / 97 \\
10 / 07 / 97 \\
10 / 09 / 97 \\
10 / 13 / 97 \\
07 / 11 / 97\end{array}$ & 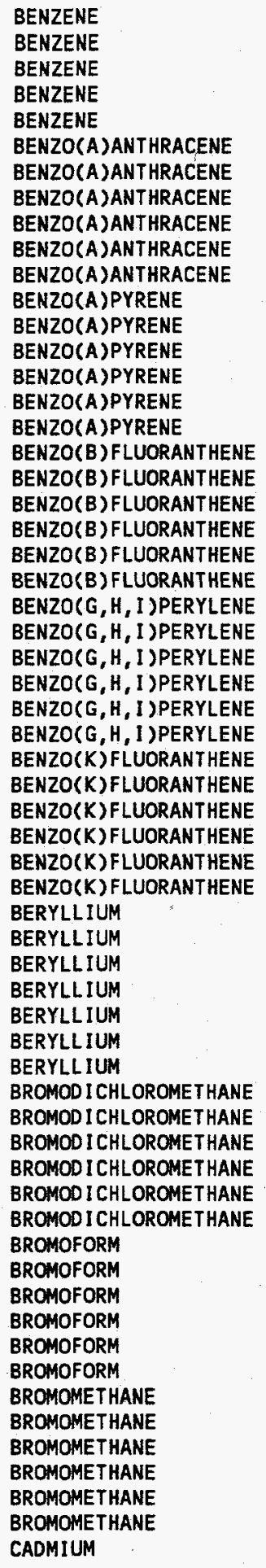 & $\begin{array}{l}\text { ND } \\
\text { ND } \\
\text { ND } \\
\text { ND } \\
\text { ND } \\
\text { ND } \\
\text { ND } \\
\text { ND } \\
\text { ND } \\
\text { ND } \\
\text { ND } \\
\text { ND } \\
\text { ND } \\
\text { ND } \\
\text { ND } \\
\text { ND } \\
\text { ND } \\
\text { ND } \\
\text { ND } \\
\text { ND } \\
\text { ND } \\
\text { ND } \\
\text { ND } \\
\text { ND } \\
\text { ND } \\
\text { ND } \\
\text { ND } \\
\text { ND } \\
\text { ND } \\
\text { ND } \\
\text { ND } \\
\text { ND } \\
\text { ND } \\
\text { ND } \\
\text { ND } \\
\text { ND } \\
\text { ND } \\
\text { ND } \\
\text { ND } \\
\text { ND } \\
\text { ND } \\
\text { ND } \\
\text { ND } \\
\text { ND } \\
\text { ND } \\
\text { ND } \\
\text { ND } \\
\text { ND } \\
\text { ND } \\
\text { ND }\end{array}$ & 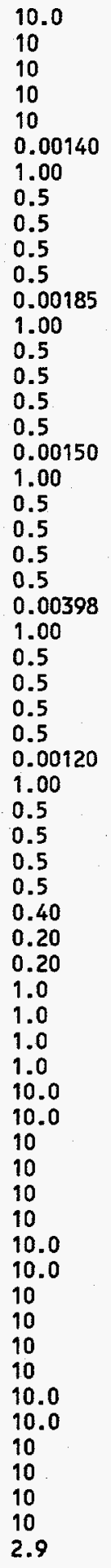 & $\begin{array}{l}U G / L \\
U G / L \\
U G / L \\
U G / L \\
U G / L \\
U G / L \\
U G / L \\
U G / L \\
U G / L \\
U G / L \\
U G / L \\
U G / L \\
U G / L \\
U G / L \\
U G / L \\
U G / L \\
U G / L \\
U G / L \\
U G / L \\
U G / L \\
U G / L \\
U G / L \\
U G / L \\
U G / L \\
U G / L \\
U G / L \\
U G / L \\
U G / L \\
U G / L \\
U G / L \\
U G / L \\
U G / L \\
U G / L \\
U G / L \\
U G / L \\
U G / L \\
U G / L \\
U G / L \\
U G / L \\
U G / L \\
U G / L \\
U G / L \\
U G / L \\
U G / L \\
U G / L \\
U G / L \\
U G / L \\
U G / L \\
U G / L \\
U G / L \\
U G / L \\
U G / L \\
U G / L \\
U G / L \\
U G / L \\
U G / L \\
U\end{array}$ & $\begin{array}{l}\text { EPA CLP } \\
\text { EPA CLP } \\
\text { EPA CLP } \\
\text { EPA CLP } \\
\text { EPA CLP } \\
\text { EPA } 8310 \\
\text { EPA } 8270 A \\
\text { EPA } 8310 \\
\text { EPA } 8310 \\
\text { SW846 } 8310 \\
\text { EPA } 8310 \\
\text { EPA } 8310 \\
\text { EPA } 8270 A \\
\text { EPA } 8310 \\
\text { EPA } 8310 \\
\text { SW846 } 8310 \\
\text { EPA } 8310 \\
\text { EPA } 8310 \\
\text { EPA } 8270 A \\
\text { EPA } 8310 \\
\text { EPA } 8310 \\
\text { SW846 } 8310 \\
\text { EPA } 8310 \\
\text { EPA } 8310 \\
\text { EPA } 8270 A \\
\text { EPA } 8310 \\
\text { EPA } 8310 \\
\text { SW846 } 8310 \\
\text { EPA } 8310 \\
\text { EPA } 8310 \\
\text { EPA } 8270 A \\
\text { EPA } 8310 \\
\text { EPA } 8310 \\
\text { SW846 } 8310 \\
\text { EPA } 8310 \\
\text { EPA CLP } \\
\text { EPA } 6010 A \\
\text { EPA } 6010 A \\
\text { EPA } 6010 A \\
\text { EPA } 6010 A \\
\text { EPA } 6010 A \\
\text { EPA } 6010 A \\
\text { EPA CLP } \\
\text { EPA CLP } \\
\text { EPA CLP } \\
\text { EPA CLP } \\
\text { EPA CLP } \\
\text { EPA CLP } \\
\text { EPA CLP } \\
\text { EPA CLP } \\
\text { EPA CLP } \\
\text { EPA CLP } \\
\text { EPA CLP } \\
\text { EPA CLP } \\
\text { EPA CLP } \\
\text { EPA CLP } \\
\text { EPA CLP } \\
\text { EPA CLP } \\
\text { EPA CLP } \\
\text { EPA CLP } \\
\text { EPA CLP }\end{array}$ \\
\hline
\end{tabular}




\begin{tabular}{|c|c|c|c|c|c|c|}
\hline WSSRAP_ID & DATE_SAM & PARAMETER & CONC & $D L$ & UNITS & METHOD \\
\hline 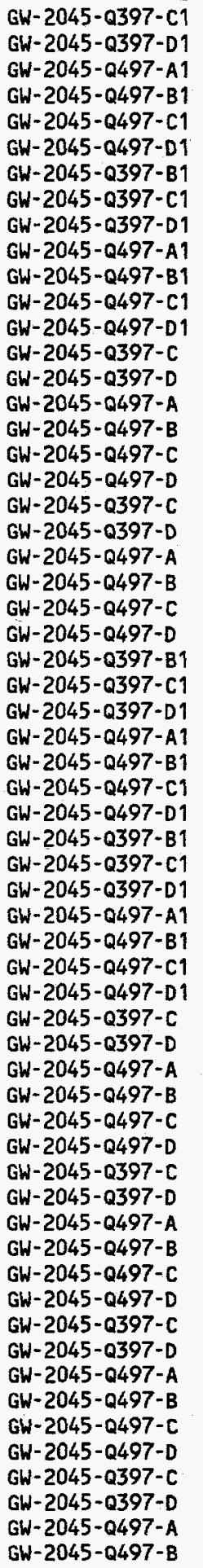 & $\begin{array}{l}07 / 15 / 97 \\
07 / 17 / 97 \\
10 / 03 / 97 \\
10 / 08 / 97 \\
10 / 10 / 97 \\
10 / 14 / 97 \\
07 / 11 / 97 \\
07 / 15 / 97 \\
07 / 17 / 97 \\
10 / 03 / 97 \\
10 / 08 / 97 \\
10 / 10 / 97 \\
10 / 14 / 97 \\
07 / 14 / 97 \\
07 / 16 / 97 \\
10 / 02 / 97 \\
10 / 07 / 97 \\
10 / 09 / 97 \\
10 / 13 / 97 \\
07 / 14 / 97 \\
07 / 16 / 97 \\
10 / 02 / 97 \\
10 / 07 / 97 \\
10 / 09 / 97 \\
10 / 13 / 97 \\
07 / 11 / 97 \\
07 / 15 / 97 \\
07 / 17 / 97 \\
10 / 03 / 97 \\
10 / 08 / 97 \\
10 / 10 / 97 \\
10 / 14 / 97 \\
07 / 11 / 97 \\
07 / 15 / 97 \\
07 / 17 / 97 \\
10 / 03 / 97 \\
10 / 08 / 97 \\
10 / 10 / 97 \\
10 / 14 / 97 \\
07 / 14 / 97 \\
07 / 16 / 97 \\
10 / 02 / 97 \\
10 / 07 / 97 \\
10 / 09 / 97 \\
10 / 13 / 97 \\
07 / 14 / 97 \\
07 / 16 / 97 \\
10 / 02 / 97 \\
10 / 07 / 97 \\
10 / 09 / 97 \\
10 / 13 / 97 \\
07 / 14 / 97 \\
07 / 16 / 97 \\
10 / 02 / 97 \\
10 / 07 / 97 \\
10 / 09 / 97 \\
10 / 13 / 97 \\
07 / 14 / 97 \\
07 / 16 / 97 \\
10 / 02 / 97 \\
10 / 07 / 97\end{array}$ & 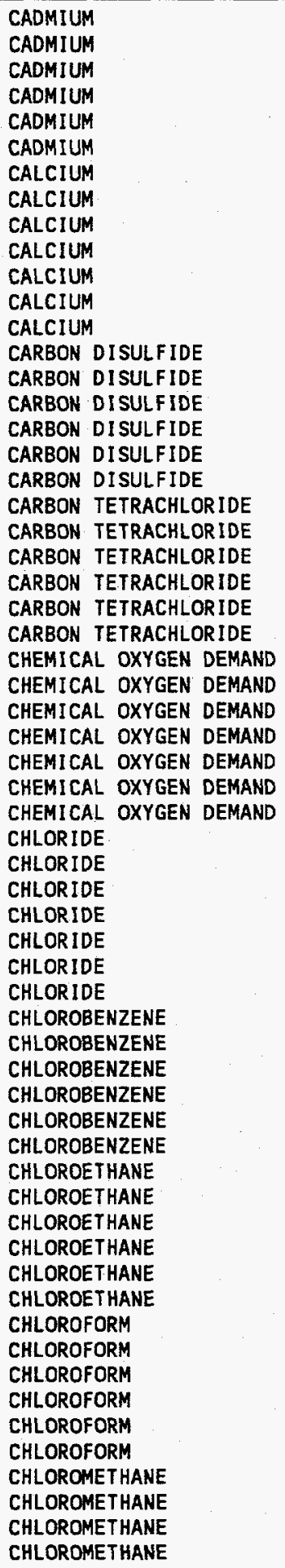 & $\begin{array}{l}\text { ND } \\
\text { ND } \\
\text { ND } \\
\text { ND } \\
\text { ND } \\
\text { ND } \\
10100 \\
95200 \\
94300 \\
10500 \\
10700 \\
10100 \\
99100 \\
\text { ND } \\
\text { ND } \\
\text { ND } \\
\text { ND } \\
\text { ND } \\
\text { ND } \\
\text { ND } \\
\text { ND } \\
\text { ND } \\
\text { ND } \\
\text { ND } \\
\text { ND } \\
6.00 \\
\text { (1.06 } \\
\text { ND } \\
\text { ND } \\
1.98 \\
\text { ND } \\
\text { ND } \\
70.7 \\
\text { 64.5 } \\
65.2 \\
66.0 \\
60.8 \\
65.0 \\
60.2 \\
\text { ND } \\
\text { ND } \\
\text { ND } \\
\text { ND } \\
\text { ND } \\
\text { ND } \\
\text { ND } \\
\text { ND } \\
\text { ND } \\
\text { ND } \\
\text { ND } \\
\text { ND } \\
\text { ND } \\
\text { (1.29 } \\
\text { (1) } \\
\text { N1) } \\
\text { ND } \\
\text { ND } \\
\text { ND } \\
\text { ND } \\
\text { ND }\end{array}$ & $\begin{array}{l}0.30 \\
0.30 \\
2.0 \\
2.0 \\
2.0 \\
2.0 \\
53.5 \\
3.0 \\
3.0 \\
13.0 \\
13.0 \\
13.0 \\
13.0 \\
10.0 \\
10.0 \\
10 \\
10 \\
10 \\
10 \\
10.0 \\
10.0 \\
10 \\
10 \\
10 \\
10 \\
5.00 \\
5.00 \\
5.00 \\
5.0 \\
5.0 \\
5.0 \\
5.0 \\
4.00 \\
0.18 \\
0.180 \\
5.00 \\
2.50 \\
2.50 \\
2.50 \\
10.0 \\
10.0 \\
10 \\
10 \\
10 \\
10 \\
10.0 \\
10.0 \\
10 \\
10 \\
10 \\
10 \\
10.0 \\
10.0 \\
10 \\
10 \\
10 \\
10 \\
10.0 \\
10.0 \\
10 \\
10 \\
\\
10 \\
\end{array}$ & 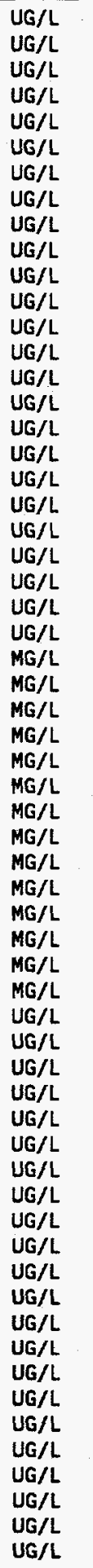 & $\begin{array}{l}\text { EPA } 6010 A \\
\text { EPA } 6010 A \\
\text { EPA } 6010 A \\
\text { EPA } 6010 A \\
\text { EPA } 6010 A \\
\text { EPA } 6010 A \\
\text { EPA CLP } \\
\text { EPA } 6010 A \\
\text { EPA } 6010 A \\
\text { EPA } 6010 A \\
\text { EPA } 6010 A \\
\text { EPA } 6010 A \\
\text { EPA } 6010 A \\
\text { EPA CLP } \\
\text { EPA CLP } \\
\text { EPA CLP } \\
\text { EPA CLP } \\
\text { EPA CLP } \\
\text { EPA CLP } \\
\text { EPA CLP } \\
\text { EPA CLP } \\
\text { EPA CLP } \\
\text { EPA CLP } \\
\text { EPA CLP } \\
\text { EPA CLP } \\
\text { EPA } 410.4 \\
\text { EPA } 410.4 \\
\text { EPA } 410.4 \\
\text { EPA } 410.0 \\
\text { EPA } 410 \\
\text { EPA } 410.0 \\
\text { EPA } 410.0 \\
\text { EPA } 300.0 \\
\text { EPA } 300.0 \\
\text { EPA } 300.0 \\
\text { EPA } 300.0 \\
\text { EPA } 300 \\
\text { EPA } 300.0 \\
\text { EPA } 300.0 \\
\text { EPA CLP } \\
\text { EPA CLP } \\
\text { EPA CLP } \\
\text { EPA CLP } \\
\text { EPA CLP } \\
\text { EPA CLP } \\
\text { EPA CLP } \\
\text { EPA CLP } \\
\text { EPA CLP } \\
\text { EPA CLP } \\
\text { EPA CLP } \\
\text { EPA CLP } \\
\text { EPA CLP } \\
\text { EPA CLP } \\
\text { EPA CLP } \\
\text { EPA CLP } \\
\text { EPA CLP } \\
\text { EPA CLP } \\
\text { EPA CLP } \\
\text { EPA CLP } \\
\text { EPA CLP } \\
\text { EPA CLP }\end{array}$ \\
\hline
\end{tabular}




\begin{tabular}{|c|c|c|c|c|c|c|}
\hline WSSRAP_ID & DATE_SAM & PARAMETER & CONC & DL & UNITS & METHOD \\
\hline $\begin{array}{l}G W-2045-Q 497-C \\
G W-2045-Q 497-D \\
G W-2045-Q 397-B 1 \\
G W-2045-Q 397-C 1 \\
G W-2045-Q 397-D 1 \\
G W-2045-Q 497-A 1 \\
G W-2045-Q 497-B 1 \\
G W-2045-Q 497-C 1 \\
G W-2045-Q 497-D 1 \\
G W-2045-Q 397-C \\
G W-2045-Q 397-D \\
G W-2045-Q 497-A \\
G W-2045-Q 497-B \\
G W-2045-Q 497-C \\
G W-2045-Q 497-D \\
G W-2045-Q 397-C \\
G W-2045-Q 397-D \\
G W-2045-Q 497-A \\
G W-2045-Q 497-B \\
G W-2045-Q 497-C \\
G W-2045-Q 497-D \\
G W-2045-Q 397-B 1 \\
G W-2045-Q 397-C 1 \\
G W-2045-Q 397-D 1 \\
G W-2045-Q 497-A 1 \\
G W-2045-Q 497-B 1 \\
G W-2045-Q 497-C 1 \\
G W-2045-Q 497-D 1 \\
G W-2045-Q 397-B 1 \\
G W-2045-Q 397-C 1 \\
G W-2045-Q 397-D 1 \\
G W-2045-Q 497-A 1 \\
G W-2045-Q 497-B 1 \\
G W-2045-Q 497-C 1 \\
G W-2045-Q 497-D 1 \\
G W-2045-Q 397-C \\
G W-2045-Q 397-D \\
G W-2045-Q 497-A \\
G W-2045-Q 497-B \\
G W-2045-Q 497-C \\
G W-2045-Q 497-D \\
G W-2045-Q 497-C \\
G W-2045-Q 397-C C \\
G W-2045-Q 397-D \\
G W-2045-Q 497-A \\
G W-2045-Q 497-B \\
G W-2045-Q 497-D \\
G W-2045-Q 397-C C \\
G W-2045-0397-D \\
G W-2045-Q 497-A \\
G W-2045-Q 497-B \\
G W-2045-Q 497-C \\
G W-2045-Q 497-D \\
G W-2045-Q 397-C \\
G W-2045-Q 397-D \\
G W-2045-Q 497-A \\
G W-2045-Q 497-B \\
G W-2045-Q 497-C \\
G W-2045-Q 497-D \\
G W-2045-Q 397-C \\
G W-2045-Q 397-D\end{array}$ & $\begin{array}{l}10 / 09 / 97 \\
10 / 13 / 97 \\
07 / 11 / 97 \\
07 / 15 / 97 \\
07 / 17 / 97 \\
10 / 03 / 97 \\
10 / 08 / 97 \\
10 / 10 / 97 \\
10 / 14 / 97 \\
07 / 14 / 97 \\
07 / 16 / 97 \\
10 / 02 / 97 \\
10 / 07 / 97 \\
10 / 09 / 97 \\
10 / 13 / 97 \\
07 / 14 / 97 \\
07 / 16 / 97 \\
10 / 02 / 97 \\
10 / 07 / 97 \\
10 / 09 / 97 \\
10 / 13 / 97 \\
07 / 11 / 97 \\
07 / 15 / 97 \\
07 / 17 / 97 \\
10 / 03 / 97 \\
10 / 08 / 97 \\
10 / 10 / 97 \\
10 / 14 / 97 \\
07 / 11 / 97 \\
07 / 15 / 97 \\
07 / 17 / 97 \\
10 / 03 / 97 \\
10 / 08 / 97 \\
10 / 10 / 97 \\
10 / 14 / 97 \\
07 / 14 / 97 \\
07 / 16 / 97 \\
10 / 02 / 97 \\
10 / 07 / 97 \\
10 / 09 / 97 \\
10 / 13 / 97 \\
10 / 09 / 97 \\
07 / 14 / 97 \\
07 / 16 / 97 \\
10 / 02 / 97 \\
10 / 07 / 97 \\
10 / 13 / 97 \\
07 / 14 / 97 \\
07 / 16 / 97 \\
10 / 02 / 97 \\
10 / 07 / 97 \\
10 / 09 / 97 \\
10 / 13 / 97 \\
07 / 14 / 97 \\
07 / 16 / 97 \\
10 / 02 / 97 \\
10 / 07 / 97 \\
10 / 09 / 97 \\
10 / 13 / 97 \\
07 / 14 / 97 \\
07 / 16 / 97\end{array}$ & 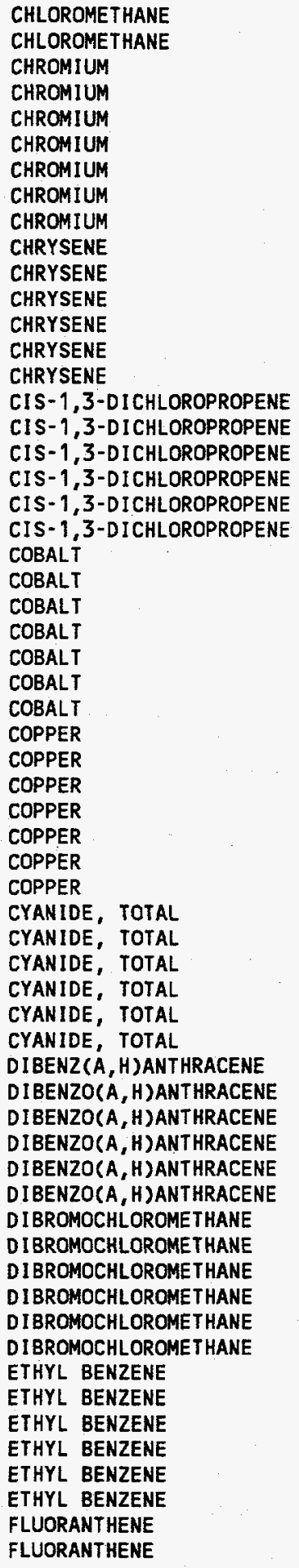 & $\begin{array}{l}\text { ND } \\
\text { ND } \\
8.7 \\
22.3 \\
15.7 \\
10.4 \\
38.5 \\
\text { ND } \\
13.9 \\
\text { ND } \\
\text { ND } \\
\text { ND } \\
\text { ND } \\
\text { ND } \\
\text { ND } \\
\text { ND } \\
\text { ND } \\
\text { ND } \\
\text { ND } \\
\text { ND } \\
7.8 \\
6.1 \\
3.2 \\
8.5 \\
7.8 \\
\text { ND } \\
\text { ND } \\
5.6 \\
4.4 \\
2.0 \\
3.7 \\
3.9 \\
\text { ND } \\
\text { ND } \\
\text { ND } \\
\text { ND } \\
\text { ND } \\
\text { ND } \\
\text { ND } \\
\text { ND } \\
\text { ND } \\
\text { ND } \\
\text { ND } \\
\text { ND } \\
\text { ND } \\
\text { ND } \\
\text { ND } \\
\text { ND } \\
\text { ND } \\
\text { ND } \\
\text { ND } \\
\text { ND } \\
\text { ND } \\
\text { ND } \\
\text { ND }\end{array}$ & 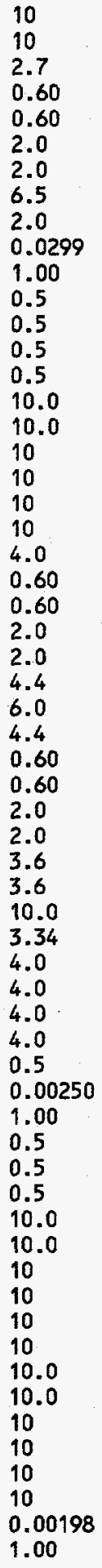 & $\begin{array}{l}U G / L \\
U G / L \\
U G / L \\
U G / L \\
U G / L \\
U G / L \\
U G / L \\
U G / L \\
U G / L \\
U G / L \\
U G / L \\
U G / L \\
U G / L \\
U G / L \\
U G / L \\
U G / L \\
U G / L \\
U G / L \\
U G / L \\
U G / L \\
U G / L \\
U G / L \\
U G / L \\
U G / L \\
U G / L \\
U G / L \\
U G / L \\
U G / L \\
U G / L \\
U G / L \\
U G / L \\
U G / L \\
U G / L \\
U G / L \\
U G / L \\
U G / L \\
U G / L \\
U G / L \\
U G / L \\
U G / L \\
U G / L \\
U G / L \\
U G / L \\
U G / L \\
U G / L \\
U G / L \\
U G / L \\
U G / L \\
U G / L \\
U G / L \\
U G / L \\
U G / L \\
U G / L \\
U G / L \\
U G / L \\
U G / L \\
U G / L \\
U G \\
U,\end{array}$ & $\begin{array}{l}\text { EPA CLP } \\
\text { EPA CLP } \\
\text { EPA CLP } \\
\text { EPA } 6010 A \\
\text { EPA } 6010 A \\
\text { EPA } 6010 A \\
\text { EPA } 6016 A \\
\text { EPA } 6010 A \\
\text { EPA } 6010 A \\
\text { EPA } 8310 \\
\text { EPA } 8270 A \\
\text { EPA } 8310 \\
\text { EPA } 8310 \\
\text { SW846 } 8310 \\
\text { EPA } 8310 \\
\text { EPA CLP } \\
\text { EPA CLP } \\
\text { EPA CLP } \\
\text { EPA CLP } \\
\text { EPA CLP } \\
\text { EPA CLP } \\
\text { EPA CLP } \\
\text { EPA } 6010 A \\
\text { EPA } 6010 A \\
\text { EPA } 6010 A \\
\text { EPA } 6010 A \\
\text { EPA } 6010 A \\
\text { EPA } 6010 A \\
\text { EPA CLP } \\
\text { EPA } 6010 A \\
\text { EPA } 6010 A \\
\text { EPA } 6010 A \\
\text { EPA } 6010 A \\
\text { EPA } 6010 A \\
\text { EPA } 6010 A \\
\text { EPA CLP } \\
\text { EPA CLP } \\
\text { EPA CLP } \\
\text { EPA CLP } \\
\text { EPA CLP } \\
\text { EPA CLP } \\
\text { SH846 } 8310 \\
\text { EPA } 8310 \\
\text { EPA } 8270 A \\
\text { EPA } 8310 \\
\text { EPA } 8310 \\
\text { EPA } 8310 \\
\text { EPA CLP } \\
\text { EPA CLP } \\
\text { EPA CLP } \\
\text { EPA CLP } \\
\text { EPA CLP } \\
\text { EPA CLP } \\
\text { EPA CLP } \\
\text { EPA CLP } \\
\text { EPA CLP } \\
\text { EPA CLP } \\
\text { EPA CLP } \\
\text { EPA CLP } \\
\text { EPA } 8310 \\
\text { EPA } 8270 A\end{array}$ \\
\hline
\end{tabular}




\begin{tabular}{|c|c|c|c|c|c|c|}
\hline WSSRAP_ID & DATE_SAM & PARAMETER & CONC & $D L$ & UNITS & METHOD \\
\hline $\begin{array}{l}G W-2045-Q 497-A \\
G W-2045-Q 497-B \\
G W-2045-Q 497-C \\
G W-2045-0497-D \\
G W-2045-Q 397-C \\
G W-2045-0397-D \\
G W-2045-Q 497-A \\
G W-2045-Q 497-B \\
G W-2045-Q 497-C \\
G W-2045-Q 497-D \\
G W-2045-Q 397-B 1 \\
G W-2045-Q 397-C 1 \\
G W-2045-Q 397-D 1 \\
G W-2045-Q 497-A 1 \\
G W-2045-Q 497-B 1 \\
G W-2045-Q 497-C 1 \\
G W-2045-Q 497-D 1 \\
G W-2045-Q 397-C \\
G W-2045-Q 397-D \\
G W-2045-Q 497-A \\
G W-2045-Q 497-B \\
G W-2045-Q 497-C \\
G W-2045-Q 497-D \\
G W-2045-Q 397-B 1 \\
G W-2045-0397-C 1 \\
G W-2045-Q 397-D 1 \\
G W-2045-Q 497-A 1 \\
G W-2045-Q 497-B 1 \\
G W-2045-Q 497-C 1 \\
G W-2045-Q 497-D 1 \\
G W-2045-Q 397-B 1 \\
G W-2045-Q 397-C 1 \\
G W-2045-0397-D 1 \\
G W-2045-0497-A 1 \\
G W-2045-Q 497-B 1 \\
G W-2045-Q 497-C 1 \\
G W-2045-Q 497-D 1 \\
G W-2045-Q 397-B 1 \\
G W-2045-Q 397-C 1 \\
G W-2045-Q 397-D 1 \\
G W-2045-Q 497-A 1 \\
G W-2045-Q 497-B 1 \\
G W-2045-Q 497-C 1 \\
G W-2045-Q 497-D 1 \\
G W-2045-Q 397-B 1 \\
G W-2045-0397-C 1 \\
G W-2045-Q 397-D 1 \\
G W-2045-Q 497-A 1 \\
G W-2045-Q 497-B 1 \\
G W-2045-Q 497-C 1 \\
G W-2045-Q 497-D 1 \\
G W-2045-Q 397-B 1 \\
G W-2045-Q 397-C 1 \\
G W-2045-Q 397-D 1 \\
G W-2045-Q 497-A 1 \\
G W-2045-Q 497-B 1 \\
G W-2045-Q 497-C 1 \\
G W-2045-Q 497-D 1 \\
G W-2045-Q 397-B 1 \\
G W-2045-0397-C 1 \\
G W-2045-0397-D 1\end{array}$ & $\begin{array}{l}10 / 02 / 97 \\
10 / 07 / 97 \\
10 / 09 / 97 \\
10 / 13 / 97 \\
07 / 14 / 97 \\
07 / 16 / 97 \\
10 / 02 / 97 \\
10 / 07 / 97 \\
10 / 09 / 97 \\
10 / 13 / 97 \\
07 / 11 / 97 \\
07 / 15 / 97 \\
07 / 17 / 97 \\
10 / 03 / 97 \\
10 / 08 / 97 \\
10 / 10 / 97 \\
10 / 14 / 97 \\
07 / 14 / 97 \\
07 / 16 / 97 \\
10 / 02 / 97 \\
10 / 07 / 97 \\
10 / 09 / 97 \\
10 / 13 / 97 \\
07 / 11 / 97 \\
07 / 15 / 97 \\
07 / 17 / 97 \\
10 / 03 / 97 \\
10 / 08 / 97 \\
10 / 10 / 97 \\
10 / 14 / 97 \\
07 / 11 / 97 \\
07 / 15 / 97 \\
07 / 17 / 97 \\
10 / 03 / 97 \\
10 / 08 / 97 \\
10 / 10 / 97 \\
10 / 14 / 97 \\
07 / 11 / 97 \\
07 / 15 / 97 \\
07 / 17 / 97 \\
10 / 03 / 97 \\
10 / 08 / 97 \\
10 / 10 / 97 \\
10 / 14 / 97 \\
07 / 11 / 97 \\
07 / 15 / 97 \\
07 / 17 / 97 \\
10 / 03 / 97 \\
10 / 08 / 97 \\
10 / 10 / 97 \\
10 / 14 / 97 \\
07 / 11 / 97 \\
07 / 15 / 97 \\
07 / 17 / 97 \\
10 / 03 / 97 \\
10 / 08 / 97 \\
10 / 10 / 97 \\
10 / 14 / 97 \\
07 / 11 / 97 \\
07 / 15 / 97 \\
07 / 17 / 97\end{array}$ & 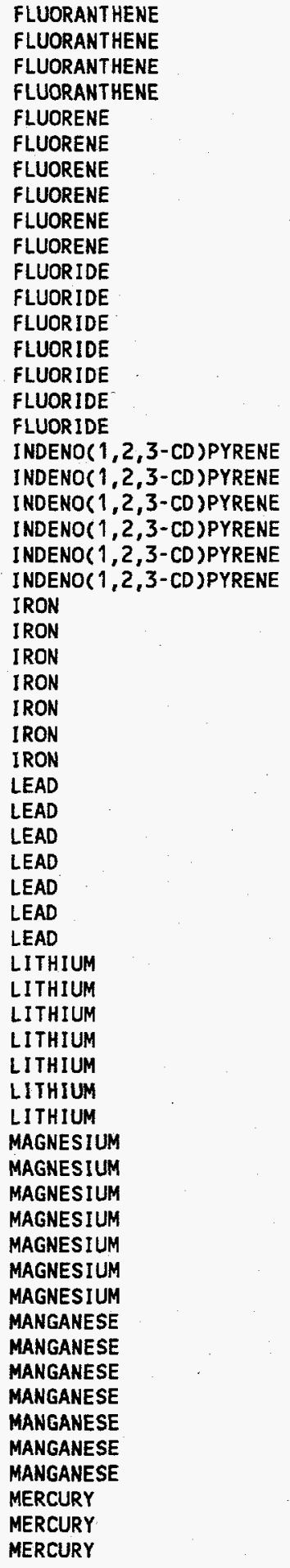 & $\begin{array}{l}\text { ND } \\
\text { ND } \\
\text { ND } \\
\text { ND } \\
\text { ND } \\
\text { ND } \\
\text { ND } \\
\text { ND } \\
\text { ND } \\
\text { ND } \\
\text { ND } \\
\text { ND } \\
0.085 \\
0.09 \\
0.13 \\
0.11 \\
\text { ND } \\
\text { ND } \\
\text { ND } \\
\text { ND } \\
\text { ND } \\
\text { ND } \\
236 \\
227 \\
\text { ND } \\
603 \\
475 \\
429 \\
362 \\
\text { ND } \\
2.7 \\
\text { ND } \\
2.1 \\
\text { ND } \\
\text { ND } \\
\text { ND } \\
\text { ND } \\
\text { ND } \\
\text { ND } \\
3.4 \\
3.6 \\
3.6 \\
3.5 \\
42600 \\
41100 \\
40500 \\
44500 \\
45800 \\
43600 \\
44100 \\
141 \\
122 \\
65.4 \\
132 \\
148 \\
86.6 \\
130 \\
\text { ND } \\
\text { ND } \\
\text { ND } \\
\end{array}$ & $\begin{array}{l}0.5 \\
0.5 \\
0.5 \\
0.5 \\
0.249 \\
1.00 \\
0.5 \\
0.5 \\
0.5 \\
0.5 \\
0.10 \\
0.0210 \\
0.0220 \\
0.05 \\
0.05 \\
0.05 \\
0.05 \\
0.00248 \\
1.00 \\
0.5 \\
0.5 \\
0.5 \\
0.5 \\
6.7 \\
2.0 \\
103 \\
61.0 \\
61.0 \\
61.0 \\
61.0 \\
1.8 \\
0.70 \\
0.70 \\
2.0 \\
2.0 \\
2.0 \\
2.0 \\
5.2 \\
11.0 \\
11.0 \\
2.0 \\
2.0 \\
2.0 \\
2.0 \\
68.0 \\
3.3 \\
3.3 \\
28.0 \\
28.0 \\
28.0 \\
28.0 \\
0.90 \\
0.30 \\
0.30 \\
2.0 \\
2.0 \\
3.0 \\
3.0 \\
0.10 \\
0.19 \\
0.17 \\
\\
\\
\end{array}$ & 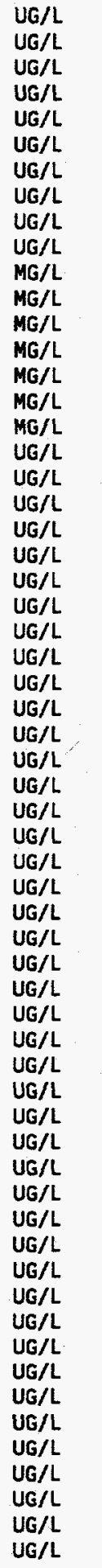 & 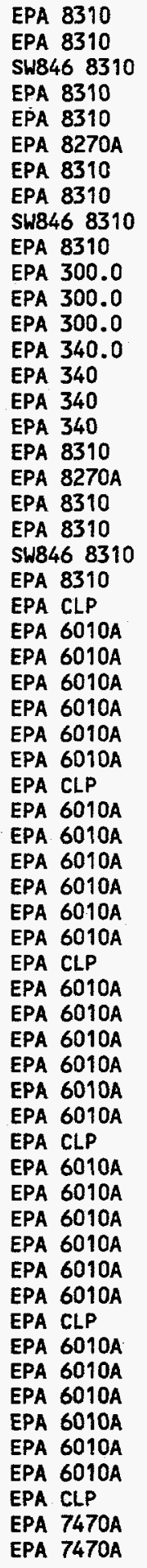 \\
\hline
\end{tabular}




\begin{tabular}{|c|c|c|c|c|c|c|}
\hline WSSRAP_ID & DATE_SAM & PARAMETER & CONC & DL & UNITS & METHOD \\
\hline 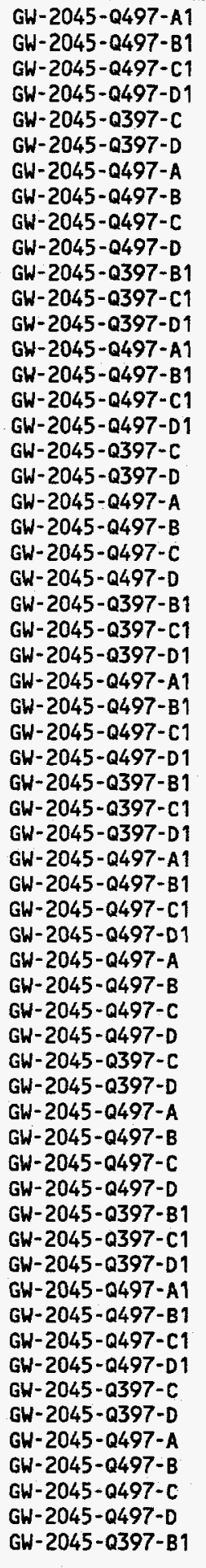 & $\begin{array}{l}10 / 03 / 97 \\
10 / 08 / 97 \\
10 / 10 / 97 \\
10 / 14 / 97 \\
07 / 14 / 97 \\
07 / 16 / 97 \\
10 / 02 / 97 \\
10 / 07 / 97 \\
10 / 09 / 97 \\
10 / 13 / 97 \\
07 / 11 / 97 \\
07 / 15 / 97 \\
07 / 17 / 97 \\
10 / 03 / 97 \\
10 / 08 / 97 \\
10 / 10 / 97 \\
10 / 14 / 97 \\
07 / 14 / 97 \\
07 / 16 / 97 \\
10 / 02 / 97 \\
10 / 07 / 97 \\
10 / 09 / 97 \\
10 / 13 / 97 \\
07 / 11 / 97 \\
07 / 15 / 97 \\
07 / 17 / 97 \\
10 / 03 / 97 \\
10 / 08 / 97 \\
10 / 10 / 97 \\
10 / 14 / 97 \\
07 / 11 / 97 \\
07 / 15 / 97 \\
07 / 17 / 97 \\
10 / 03 / 97 \\
10 / 08 / 97 \\
10 / 10 / 97 \\
10 / 14 / 97 \\
10 / 02 / 97 \\
10 / 07 / 97 \\
10 / 09 / 97 \\
10 / 13 / 97 \\
07 / 14 / 97 \\
07 / 16 / 97 \\
10 / 02 / 97 \\
10 / 07 / 97 \\
10 / 09 / 97 \\
10 / 13 / 97 \\
07 / 11 / 97 \\
07 / 15 / 97 \\
07 / 17 / 97 \\
10 / 03 / 97 \\
10 / 08 / 97 \\
10 / 10 / 97 \\
10 / 14 / 97 \\
07 / 14 / 97 \\
07 / 16 / 97 \\
10 / 02 / 97 \\
10 / 07 / 97 \\
10 / 09 / 97 \\
10 / 13 / 97 \\
07 / 11 / 97\end{array}$ & 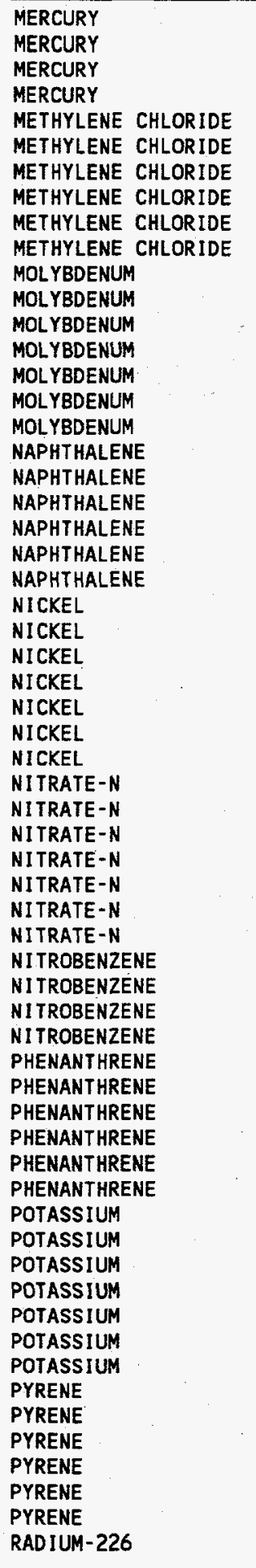 & $\begin{array}{l}\text { ND } \\
\text { ND } \\
\text { ND } \\
\text { ND } \\
\text { ND } \\
(2.67 \\
\text { (2) } \\
\text { ND } \\
42 \\
\text { ND } \\
\text { ND } \\
5.2 \\
5.1 \\
\text { ND } \\
\text { ND } \\
\text { ND } \\
\text { ND } \\
\text { ND } \\
\text { ND } \\
\text { ND } \\
\text { ND } \\
\text { ND } \\
\text { ND } \\
443 \\
424 \\
215 \\
548 \\
607 \\
298 \\
484 \\
1.34 \\
1.48 \\
1.57 \\
1.78 \\
1.70 \\
1.80 \\
1.71 \\
\text { ND } \\
\text { ND } \\
\text { ND } \\
\text { ND } \\
\text { ND } \\
\text { ND } \\
\text { ND } \\
\text { ND } \\
\text { ND } \\
\text { ND } \\
3510 \\
2480 \\
2480 \\
3310 \\
3220 \\
2490 \\
2310 \\
\text { ND } \\
\text { ND } \\
\text { ND } \\
\text { ND } \\
\text { ND } \\
\text { ND } \\
0.199\end{array}$ & $\begin{array}{l}0.10 \\
0.10 \\
0.10 \\
0.10 \\
10.0 \\
10.0 \\
10 \\
10 \\
10 \\
10 \\
7.3 \\
0.70 \\
0.70 \\
7.0 \\
7.0 \\
7.0 \\
7.0 \\
0.600 \\
1.00 \\
0.5 \\
0.5 \\
0.5 \\
0.5 \\
14.8 \\
1.0 \\
1.0 \\
2.0 \\
2.0 \\
2.0 \\
2.0 \\
0.25 \\
0.00700 \\
0.00700 \\
0.100 \\
0.100 \\
0.100 \\
0.100 \\
0.030 \\
0.030 \\
0.030 \\
0.030 \\
0.0744 \\
1.00 \\
0.5 \\
0.5 \\
0.5 \\
0.5 \\
1770 \\
11.0 \\
11.0 \\
69.0 \\
94.0 \\
69.0 \\
69.0 \\
0.0278 \\
1.00 \\
0.5 \\
0.5 \\
0.5 \\
0.5 \\
0.152 \\
0\end{array}$ & 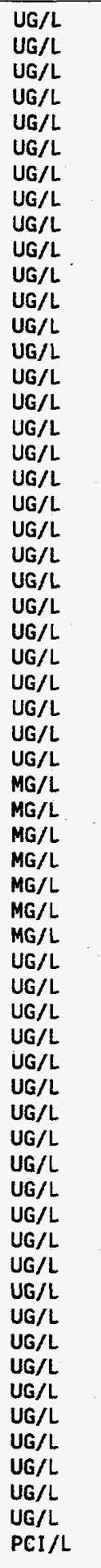 & $\begin{array}{l}\text { EPA } 7470 A \\
\text { EPA } 7470 \\
\text { EPA } 7470 A \\
\text { EPA } 7470 A \\
\text { EPA CLP } \\
\text { EPA CLP } \\
\text { EPA CLP } \\
\text { EPA CLP } \\
\text { EPA CLP } \\
\text { EPA CLP } \\
\text { EPA CLP } \\
\text { EPA } 6010 A \\
\text { EPA } 6010 A \\
\text { EPA } 6010 A \\
\text { EPA } 6010 A \\
\text { EPA } 6010 A \\
\text { EPA } 6010 A \\
\text { EPA } 8310 \\
\text { EPA } 8270 A \\
\text { EPA } 8310 \\
\text { EPA } 8310 \\
\text { SW846 } 8310 \\
\text { EPA } 8310 \\
\text { EPA CLP } \\
\text { EPA } 6010 A \\
\text { EPA } 6010 A \\
\text { EPA } 6010 A \\
\text { EPA } 6010 A \\
\text { EPA } 6010 A \\
\text { EPA } 6010 A \\
\text { EPA } 353.1 \\
\text { EPA } 353.1 \\
\text { EPA } 353.1 \\
\text { EPA } 353.1 \\
\text { EPA } 353.1 \\
\text { EPA } 353.1 \\
\text { EPA } 353.1 \\
\text { USATHAMA } \\
\text { USATHAMA } \\
\text { USATHAMA } \\
\text { USATHAMA } \\
\text { EPA } 8310 \\
\text { EPA } 8270 A \\
\text { EPA } 8310 \\
\text { EPA } 8310 \\
\text { SW846 } 8310 \\
\text { EPA } 8310 \\
\text { EPA } C L P \\
\text { EPA } 6010 A \\
\text { EPA } 6010 A \\
\text { EPA } 6010 A \\
\text { EPA } 6010 A \\
\text { EPA } 6010 A \\
\text { EPA } 6010 A \\
\text { EPA } 8310 \\
\text { EPA } 8270 A \\
\text { EPA } 8310 \\
\text { EPA } 8310 \\
\text { SW846 } 8310 \\
\text { EPA } 8310 \\
\text { EPA } 904.0 \\
\end{array}$ \\
\hline
\end{tabular}




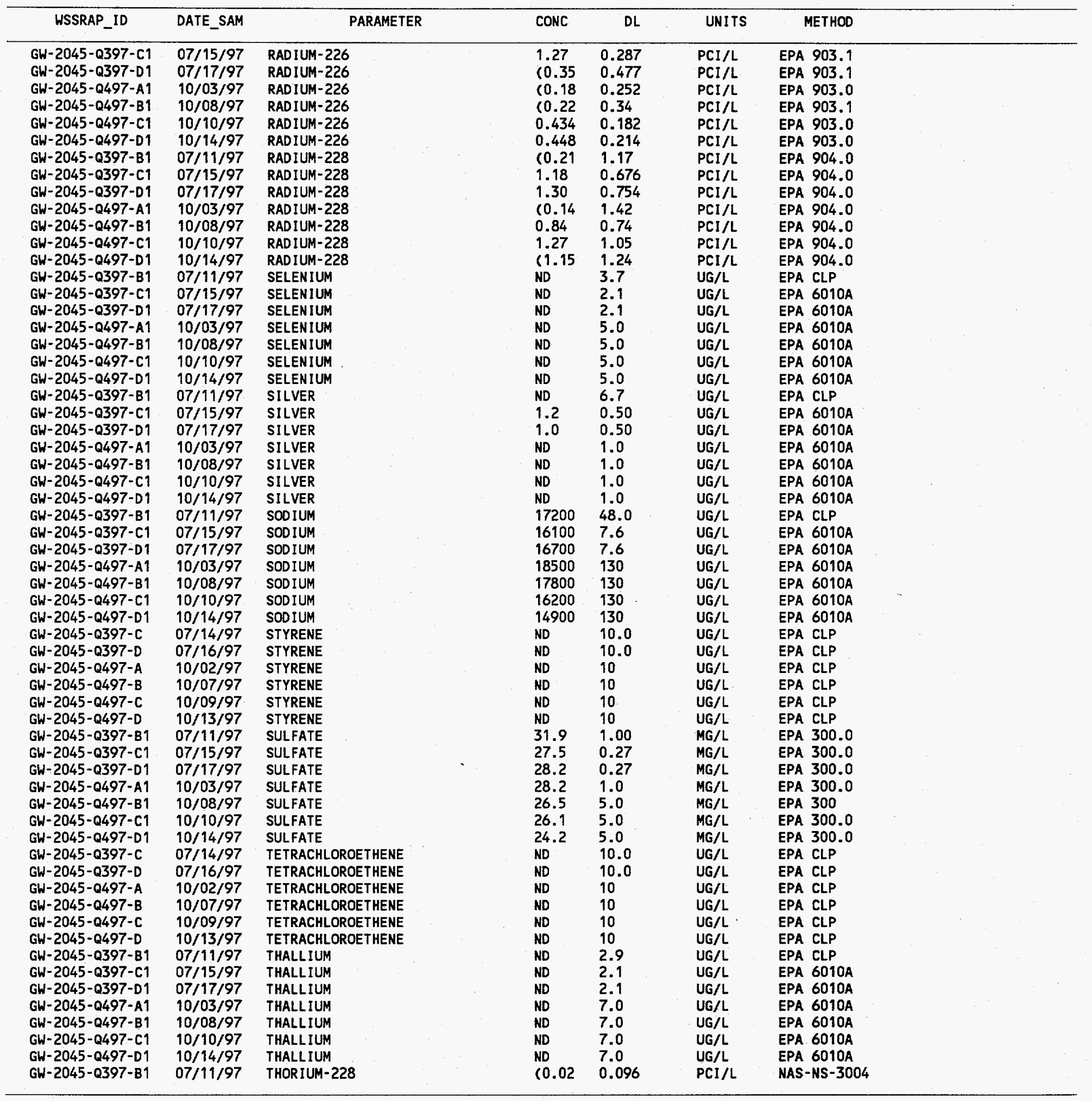




\begin{tabular}{|c|c|c|c|c|c|c|}
\hline WSSRAP_ID & DATE_SAM & PARAMETER & CONC & $D L$ & UNITS & METHOD \\
\hline 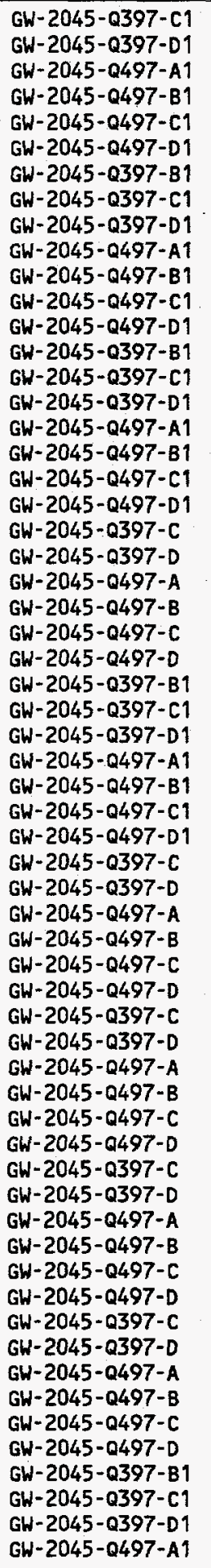 & $\begin{array}{l}07 / 15 / 97 \\
07 / 17 / 97 \\
10 / 03 / 97 \\
10 / 08 / 97 \\
10 / 10 / 97 \\
10 / 14 / 97 \\
07 / 11 / 97 \\
07 / 15 / 97 \\
07 / 17 / 97 \\
10 / 03 / 97 \\
10 / 08 / 97 \\
10 / 10 / 97 \\
10 / 14 / 97 \\
07 / 11 / 97 \\
07 / 15 / 97 \\
07 / 17 / 97 \\
10 / 03 / 97 \\
10 / 08 / 97 \\
10 / 10 / 97 \\
10 / 14 / 97 \\
07 / 14 / 97 \\
07 / 16 / 97 \\
10 / 02 / 97 \\
10 / 07 / 97 \\
10 / 09 / 97 \\
10 / 13 / 97 \\
07 / 11 / 97 \\
07 / 15 / 97 \\
07 / 17 / 97 \\
10 / 03 / 97 \\
10 / 08 / 97 \\
10 / 10 / 97 \\
10 / 14 / 97 \\
07 / 14 / 97 \\
07 / 16 / 97 \\
10 / 02 / 97 \\
10 / 07 / 97 \\
10 / 09 / 97 \\
10 / 13 / 97 \\
07 / 14 / 97 \\
07 / 16 / 97 \\
10 / 02 / 97 \\
10 / 07 / 97 \\
10 / 09 / 97 \\
10 / 13 / 97 \\
07 / 14 / 97 \\
07 / 16 / 97 \\
10 / 02 / 97 \\
10 / 07 / 97 \\
10 / 09 / 97 \\
10 / 13 / 97 \\
07 / 14 / 97 \\
07 / 16 / 97 \\
10 / 02 / 97 \\
10 / 07 / 97 \\
10 / 09 / 97 \\
10 / 13 / 97 \\
07 / 11 / 97 \\
07 / 15 / 97 \\
07 / 17 / 97 \\
10 / 03 / 97\end{array}$ & 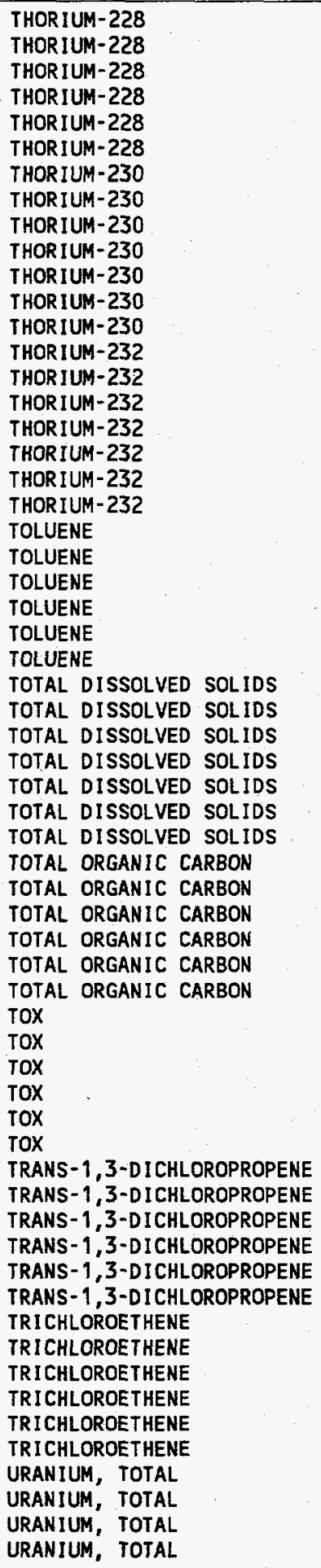 & $\begin{array}{l}\text { ND } \\
C 0.02 \\
C 0.42 \\
\text { ND } \\
C 0.46 \\
C 0.30 \\
C 0.06 \\
C 0.01 \\
C 0.03 \\
0.747 \\
C 0.00 \\
C 0.45 \\
C 0.23 \\
C 0.00 \\
\text { ND } \\
\text { ND } \\
C 0.08 \\
C 0.03 \\
\text { ND } \\
\text { ND } \\
\text { ND } \\
\text { ND } \\
\text { ND } \\
\text { ND } \\
\text { ND } \\
\text { ND } \\
534 \\
515 \\
510 \\
542 \\
406 \\
504 \\
506 \\
3.70 \\
1.24 \\
12.2 \\
39.8 \\
31.6 \\
\text { ND } \\
0.028 \\
0.005 \\
0.024 \\
0.011 \\
0.027 \\
0.009 \\
\text { ND } \\
\text { ND } \\
\text { ND } \\
\text { ND } \\
\text { ND } \\
\text { ND } \\
\text { ND } \\
\text { ND } \\
\text { ND } \\
\text { ND } \\
\text { ND } \\
\text { ND } \\
0.917 \\
0.826 \\
0.833 \\
0.870\end{array}$ & $\begin{array}{l}0.180 \\
0.190 \\
0.500 \\
0.22 \\
0.675 \\
0.388 \\
0.075 \\
0.0757 \\
0.110 \\
0.501 \\
0.077 \\
0.550 \\
0.322 \\
0.045 \\
0.0430 \\
0.0941 \\
0.449 \\
0.077 \\
0.037 \\
0.321 \\
10.0 \\
10.0 \\
10 \\
10 \\
10 \\
10 \\
10 \\
5\end{array}$ & 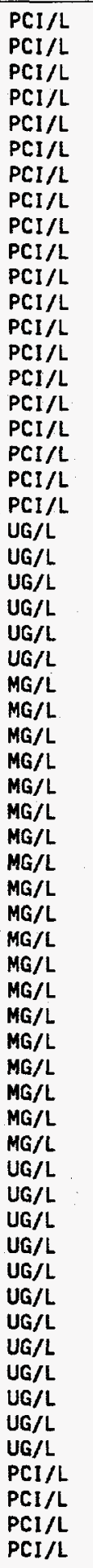 & $\begin{array}{l}\text { HASL } 300 \\
\text { HASL } 300 \\
\text { EML TH-01 } \\
\text { EPA } 907.0 \\
\text { EML TH-01 } \\
\text { EML TH-01 } \\
\text { NAS-NS-3004 } \\
\text { HASL } 300 \\
\text { HASL } 300 \\
\text { EML TH-01 } \\
\text { EPA } 907.0 \\
\text { EML TH-01 } \\
\text { EML TH-01 } \\
\text { NAS-NS-3004 } \\
\text { HASL } 300 \\
\text { HASL } 300 \\
\text { EML TH-01 } \\
\text { EPA } 907.0 \\
\text { EML TH-01 } \\
\text { EML TH-01 } \\
\text { EPA CLP } \\
\text { EPA CLP } \\
\text { EPA CLP } \\
\text { EPA CLP } \\
\text { EPA CLP } \\
\text { EPA CLP } \\
\text { EPA } 160.1 \\
\text { EPA } 160.1 \\
\text { EPA } 160.1 \\
\text { EPA } 160.1 \\
\text { EPA } 160.1 \\
\text { EPA } 160.1 \\
\text { EPA } 160.1 \\
\text { EPA } 415.1 \\
\text { EPA } 415.1 \\
\text { EPA } 415.1 \\
\text { EPA } 415.1 \\
\text { EPA } 415.1 \\
\text { EPA } 415.1 \\
\text { EPA } 9020 A \\
\text { EPA } 90208 \\
\text { EPA } 9020 A \\
\text { EPA } 9020 A \\
\text { EPA } 9020 A \\
\text { EPA } 9020 A \\
\text { EPA CLP } \\
\text { EPA CLP } \\
\text { EPA CLP } \\
\text { EPA CLP } \\
\text { EPA CLP } \\
\text { EPA CLP } \\
\text { EPA CLP } \\
\text { EPA CLP } \\
\text { EPA CLP } \\
\text { EPA CLP } \\
\text { EPA CLP } \\
\text { EPA CLP } \\
\text { ASTM } 5174-91 \\
\text { ASTM } 5174-91 \\
\text { ASTM } 5174-91 \\
\text { ASTM } 5174-91\end{array}$ \\
\hline
\end{tabular}




\begin{tabular}{|c|c|c|c|c|c|c|}
\hline WSSRAP_ID & DATE_SAM & PARAMETER & CONC & $D L$ & UNITS & METHOD \\
\hline 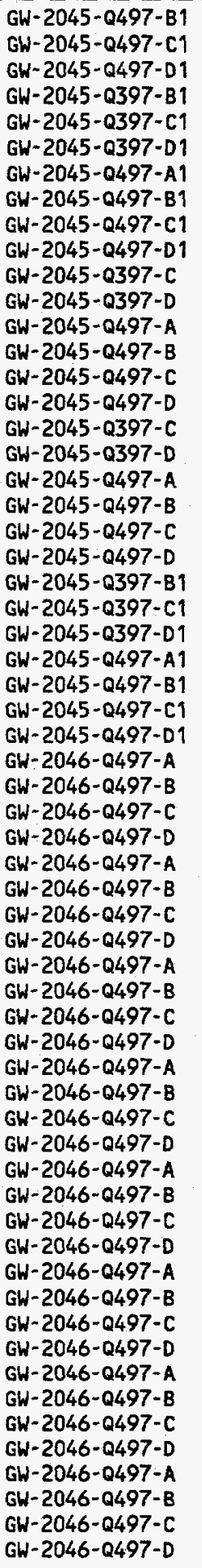 & $\begin{array}{l}10 / 08 / 97 \\
10 / 10 / 97 \\
10 / 14 / 97 \\
07 / 11 / 97 \\
07 / 15 / 97 \\
07 / 17 / 97 \\
10 / 03 / 97 \\
10 / 08 / 97 \\
10 / 10 / 97 \\
10 / 14 / 97 \\
07 / 14 / 97 \\
07 / 16 / 97 \\
10 / 02 / 97 \\
10 / 07 / 97 \\
10 / 09 / 97 \\
10 / 13 / 97 \\
07 / 14 / 97 \\
07 / 16 / 97 \\
10 / 02 / 97 \\
10 / 07 / 97 \\
10 / 09 / 97 \\
10 / 13 / 97 \\
07 / 11 / 97 \\
07 / 15 / 97 \\
07 / 17 / 97 \\
10 / 03 / 97 \\
10 / 08 / 97 \\
10 / 10 / 97 \\
10 / 14 / 97 \\
10 / 02 / 97 \\
10 / 07 / 97 \\
10 / 08 / 97 \\
10 / 09 / 97 \\
10 / 02 / 97 \\
10 / 07 / 97 \\
10 / 08 / 97 \\
10 / 09 / 97 \\
10 / 02 / 97 \\
10 / 07 / 97 \\
10 / 08 / 97 \\
10 / 09 / 97 \\
10 / 02 / 97 \\
10 / 07 / 97 \\
10 / 08 / 97 \\
10 / 09 / 97 \\
10 / 02 / 97 \\
10 / 07 / 97 \\
10 / 08 / 97 \\
10 / 09 / 97 \\
10 / 02 / 97 \\
10 / 07 / 97 \\
10 / 08 / 97 \\
10 / 09 / 97 \\
10 / 02 / 97 \\
10 / 07 / 97 \\
10 / 08 / 97 \\
10 / 09 / 97 \\
10 / 02 / 97 \\
10 / 07 / 97 \\
10 / 08 / 97 \\
10 / 09 / 97\end{array}$ & 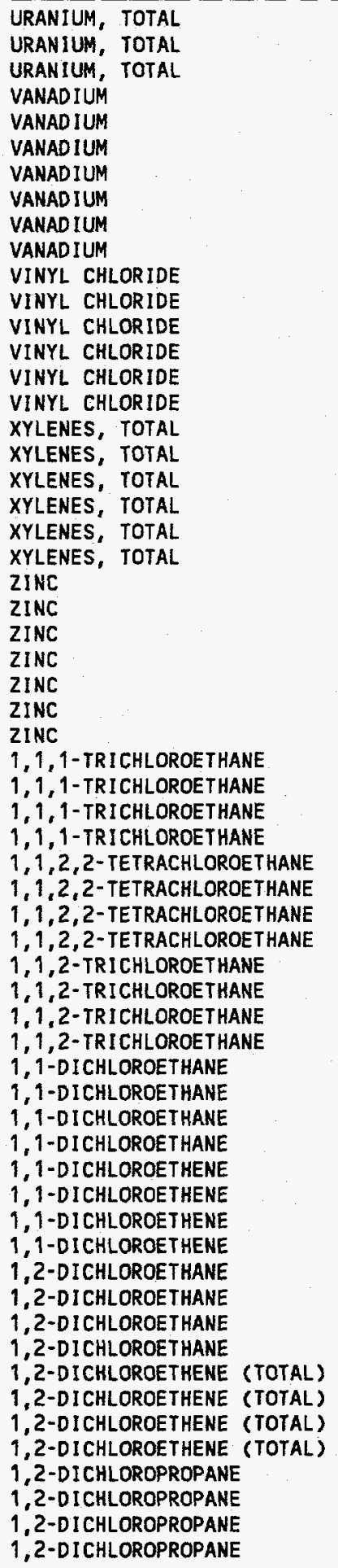 & 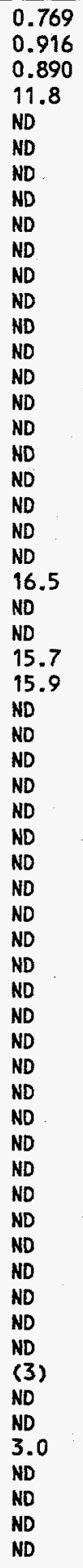 & 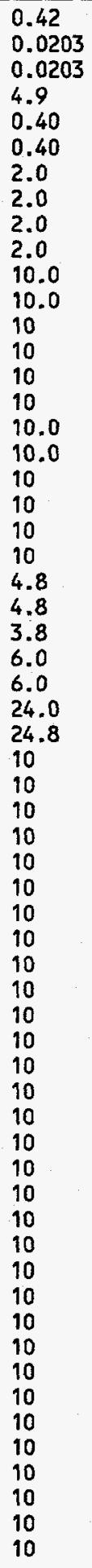 & $\begin{array}{l}P C I / L \\
P C I / L \\
P C I / L \\
U G / L \\
U G / L \\
U G / L \\
U G / L \\
U G / L \\
U G / L \\
U G / L \\
U G / L \\
U G / L \\
U G / L \\
U G / L \\
U G / L \\
U G / L \\
U G / L \\
U G / L \\
U G / L \\
U G / L \\
U G / L \\
U G / L \\
U G / L \\
U G / L \\
U G / L \\
U G / L \\
U G / L \\
U G / L \\
U G / L \\
U G / L \\
U G / L \\
U G / L \\
U G / L \\
U G / L \\
U G / L \\
U G / L \\
U G / L \\
U G / L \\
U G / L \\
U G / L \\
U G / L \\
U G / L \\
U G / L \\
U G / L \\
U G / L \\
U G / L \\
U G / L \\
U G / L \\
U G / L \\
U G / L \\
U G / L \\
U G / L \\
U G / L \\
U G / L \\
U G / L \\
\text { UG/L } \\
\text { UG }\end{array}$ & $\begin{array}{l}\text { ASTM } 5174-91 \\
\text { ASTM } 5174-91 \\
\text { ASTM } 5174-91 \\
\text { EPA CLP } \\
\text { EPA } 6010 A \\
\text { EPA } 6010 A \\
\text { EPA } 6010 A \\
\text { EPA } 6010 A \\
\text { EPA } 6010 A \\
\text { EPA } 6010 A \\
\text { EPA CLP } \\
\text { EPA CLP } \\
\text { EPA CLP } \\
\text { EPA CLP } \\
\text { EPA CLP } \\
\text { EPA CLP } \\
\text { EPA CLP } \\
\text { EPA CLP } \\
\text { EPA CLP } \\
\text { EPA CLP } \\
\text { EPA CLP } \\
\text { EPA CLP } \\
\text { EPA CLP } \\
\text { EPA } 6010 A \\
\text { EPA } 6010 A \\
\text { EPA } 6010 A \\
\text { EPA } 6010 A \\
\text { EPA } 6010 A \\
\text { EPA } 6010 A \\
\text { EPA CLP } \\
\text { EPA CLP } \\
\text { EPA CLP } \\
\text { EPA CLP } \\
\text { EPA CLP } \\
\text { EPA CLP } \\
\text { EPA CLP } \\
\text { EPA CLP } \\
\text { EPA CLP } \\
\text { EPA CLP } \\
\text { EPA CLP } \\
\text { EPA CLP } \\
\text { EPA CLP } \\
\text { EPA CLP } \\
\text { EPA CLP } \\
\text { EPA CLP } \\
\text { EPA CLP } \\
\text { EPA CLP } \\
\text { EPA CLP } \\
\text { EPA CLP } \\
\text { EPA CLP } \\
\text { EPA CLP } \\
\text { EPA CLP } \\
\text { EPA CLP } \\
\text { EPA CLP } \\
\text { EPA CLP } \\
\text { EPA CLP } \\
\text { EPA CLP } \\
\text { EPA CLP } \\
\text { EPA CLP } \\
\text { EPA CLP } \\
\text { EPA CLP }\end{array}$ \\
\hline
\end{tabular}




\begin{tabular}{|c|c|c|c|c|c|c|}
\hline WSSRAP_ID & DATE_SAM & PARAMETER & CONC & $\mathrm{DL}$ & UNITS & METHOD \\
\hline 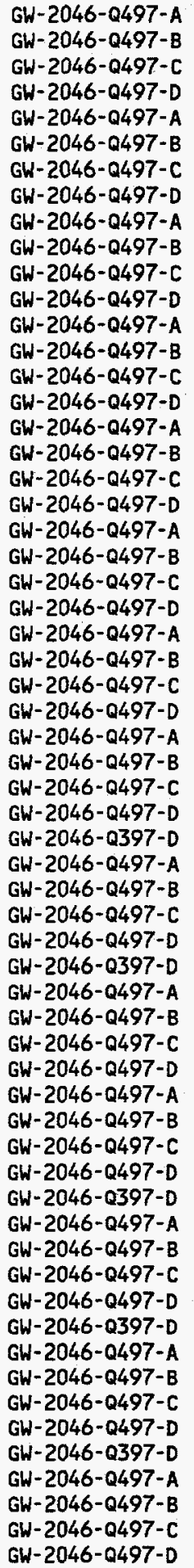 & $\begin{array}{l}10 / 02 / 97 \\
10 / 07 / 97 \\
10 / 08 / 97 \\
10 / 09 / 97 \\
10 / 02 / 97 \\
10 / 07 / 97 \\
10 / 08 / 97 \\
10 / 09 / 97 \\
10 / 02 / 97 \\
10 / 07 / 97 \\
10 / 08 / 97 \\
10 / 09 / 97 \\
10 / 02 / 97 \\
10 / 07 / 97 \\
10 / 08 / 97 \\
10 / 09 / 97 \\
10 / 02 / 97 \\
10 / 07 / 97 \\
10 / 08 / 97 \\
10 / 09 / 97 \\
10 / 02 / 97 \\
10 / 07 / 97 \\
10 / 08 / 97 \\
10 / 09 / 97 \\
10 / 02 / 97 \\
10 / 07 / 97 \\
10 / 08 / 97 \\
10 / 09 / 97 \\
10 / 02 / 97 \\
10 / 07 / 97 \\
10 / 08 / 97 \\
10 / 09 / 97 \\
07 / 11 / 97 \\
10 / 02 / 97 \\
10 / 07 / 97 \\
10 / 08 / 97 \\
10 / 09 / 97 \\
07 / 11 / 97 \\
10 / 02 / 97 \\
10 / 07 / 97 \\
10 / 08 / 97 \\
10 / 09 / 97 \\
10 / 02 / 97 \\
10 / 07 / 97 \\
10 / 08 / 97 \\
10 / 09 / 97 \\
07 / 11 / 97 \\
10 / 02 / 97 \\
10 / 07 / 97 \\
10 / 08 / 97 \\
10 / 09 / 97 \\
07 / 11 / 97 \\
10 / 02 / 97 \\
10 / 07 / 97 \\
10 / 08 / 97 \\
10 / 09 / 97 \\
07 / 11 / 97 \\
10 / 02 / 97 \\
10 / 07 / 97 \\
10 / 08 / 97 \\
10 / 09 / 97\end{array}$ & 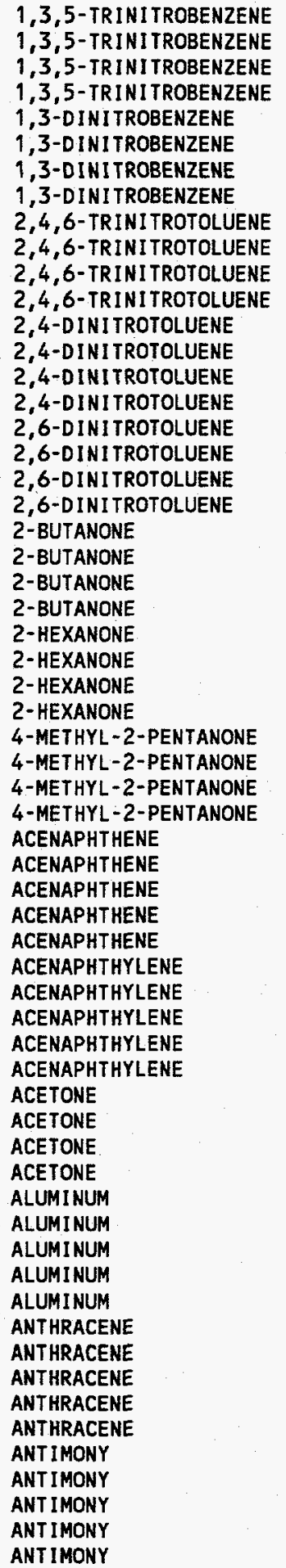 & $\begin{array}{l}2.4 \\
2.5 \\
2.2 \\
2.2 \\
\text { ND } \\
\text { C.06 } \\
0.08 \\
\text { C0.07 } \\
2.3 \\
2.4 \\
2.2 \\
2.1 \\
0.18 \\
0.17 \\
0.21 \\
0.32 \\
14.0 \\
13 \\
16 \\
\text { 15 } \\
\text { ND } \\
\text { ND } \\
\text { ND } \\
\text { ND } \\
\text { ND } \\
\text { ND } \\
\text { ND } \\
\text { ND } \\
\text { ND } \\
\text { ND } \\
\text { ND } \\
\text { ND } \\
\text { ND } \\
\text { ND } \\
\text { ND } \\
\text { ND } \\
\text { ND } \\
\text { ND } \\
\text { ND } \\
\text { ND } \\
\text { ND } \\
\text { ND } \\
\text { ND } \\
\text { ND } \\
\text { ND } \\
\text { ND } \\
\text { ND } \\
247 \\
154 \\
91.4 \\
262 \\
\text { ND } \\
\text { ND } \\
\text { ND } \\
\text { ND } \\
\text { ND } \\
\text { ND } \\
\text { ND } \\
\text { ND } \\
\text { ND } \\
\text { ND }\end{array}$ & $\begin{array}{l}0.030 \\
0.030 \\
0.030 \\
0.030 \\
0.09 \\
0.090 \\
0.090 \\
0.090 \\
0.03 \\
0.030 \\
0.030 \\
0.030 \\
0.03 \\
0.030 \\
0.030 \\
0.030 \\
0.010 \\
0.010 \\
0.010 \\
0.010 \\
10 \\
10 \\
10 \\
10 \\
10 \\
10 \\
10 \\
10 \\
10 \\
10 \\
10 \\
10 \\
5.0 \\
0.5 \\
0.5 \\
0.5 \\
0.5 \\
5.0 \\
0.5 \\
0.5 \\
0.5 \\
0.5 \\
10 \\
10 \\
10 \\
10 \\
24.7 \\
27.0 \\
27.0 \\
27.0 \\
27.0 \\
5.0 \\
0.5 \\
0.5 \\
0.5 \\
0.5 \\
42.5 \\
11.0 \\
11.0 \\
11.0 \\
11.0\end{array}$ & $\begin{array}{l}U G / L \\
U G / L \\
U G / L \\
U G / L \\
U G / L \\
U G / L \\
U G / L \\
U G / L \\
U G / L \\
U G / L \\
U G / L \\
U G / L \\
U G / L \\
U G / L \\
U G / L \\
U G / L \\
U G / L \\
U G / L \\
U G / L \\
U G / L \\
U G / L \\
U G / L \\
U G / L \\
U G / L \\
U G / L \\
U G / L \\
U G / L \\
U G / L \\
U G / L \\
U G / L \\
U G / L \\
U G / L \\
U G / L \\
U G / L \\
U G / L \\
U G / L \\
U G / L \\
U G / L \\
U G / L \\
U G / L \\
U G / L \\
U G / L \\
U G / L \\
U G / L \\
U G / L \\
U G / L \\
U G / L \\
U G / L \\
U G / L \\
U G / L \\
U G / L \\
U G / L \\
U G / L \\
U G / L \\
U G / L \\
U G / L \\
U G / L \\
U G / L \\
U G / L \\
U G / L \\
U G / L\end{array}$ & $\begin{array}{l}\text { USATHAMA } \\
\text { USATHAMA } \\
\text { USATHAMA } \\
\text { USATHAMA } \\
\text { USATHAMA } \\
\text { USATHAMA } \\
\text { USATHAMA } \\
\text { USATHAMA } \\
\text { USATHAMA } \\
\text { USATHAMA } \\
\text { USATHAMA } \\
\text { USATHAMA } \\
\text { USATHAMA } \\
\text { USATHAMA } \\
\text { USATHAMA } \\
\text { USATHAMA } \\
\text { USATHAMA } \\
\text { USATHAMA } \\
\text { USATHAMA } \\
\text { USATHAMA } \\
\text { EPA CLP } \\
\text { EPA CLP } \\
\text { EPA CLP } \\
\text { EPA CLP } \\
\text { EPA CLP } \\
\text { EPA CLP } \\
\text { EPA CLP } \\
\text { EPA CLP } \\
\text { EPA CLP } \\
\text { EPA } \\
\text { EPA CLP } 8310 \\
\text { EPA } 6010 A \\
\text { EPA } 6010 A \\
\text { EPA } 601010 \text { EPA } 6010 \\
\text { EPA CLP } \\
\text { EPA } 6010 A \\
\text { EPA } 8310 \\
\text { EPA } 8310 \\
\text { EPA CLP } \\
\text { EPA } 8310 \\
\text { EPA } 8310 \\
\text { EPA } 8310 \\
\text { EPA } 8310 \\
\text { EPA } 8310 \\
\text { EPA } 8310 \\
\text { EPA } 8310 \\
\text { SW846 } 8310 \\
\text { SW846 } 8310 \\
\text { EPA CLP } \\
\text { EPA CLP } \\
\text { EPA CLP } \\
\text { EA } \\
\text { EA } \\
\text { EA } \\
\text { EA }\end{array}$ \\
\hline
\end{tabular}




\begin{tabular}{|c|c|c|c|c|c|c|}
\hline WSSRAP_ID & DATE_SAM & PARAMETER & CONC & $D L$ & UNITS & METHOD \\
\hline $\begin{array}{l}G W-2046-Q 397-D \\
G W-2046-Q 497-A \\
G W-2046-Q 497-B \\
G W-2046-Q 497-C \\
G W-2046-Q 497-D \\
G W-2046-0397-D \\
G W-2046-Q 497-A \\
G W-2046-Q 497-B \\
G W-2046-Q 497-C \\
G W-2046-Q 497-D \\
G W-2046-Q 397-D \\
G W-2046-Q 497-A \\
G W-2046-Q 497-B \\
G W-2046-Q 497-C \\
G W-2046-Q 497-D \\
G W-2046-Q 397-D \\
G W-2046-Q 497-A \\
G W-2046-Q 497-B \\
G W-2046-Q 497-C \\
G W-2046-Q 497-D \\
G W-2046-Q 397-D \\
G W-2046-Q 497-A \\
G W-2046-Q 497-B \\
G W-2046-Q 497-C \\
G W-2046-Q 497-D \\
G W-2046-Q 397-D \\
G W-2046-Q 497-A \\
G W-2046-Q 497-B \\
G W-2046-Q 497-C \\
G W-2046-Q 497-D \\
G W-2046-Q 397-D \\
G W-2046-Q 497-A \\
G W-2046-Q 497-B \\
G W-2046-0497-C \\
G W-2046-Q 497-D \\
G W-2046-Q 397-D \\
G W-2046-0497-A \\
G W-2046-Q 497-B \\
G W-2046-Q 497-C \\
G W-2046-Q 497-D \\
G W-2046-Q 397-D \\
G W-2046-Q 497-A \\
G W-2046-Q 497-B \\
G W-2046-Q 497-C \\
G W-2046-Q 497-D \\
G W-2046-Q 497-A \\
G W-2046-Q 497-B \\
G W-2046-0497-C \\
G W-2046-0497-D \\
G W-2046-0397-D \\
G W-2046-Q 497-A \\
G W-2046-Q 497-B \\
G W-2046-Q 497-C \\
G W-2046-Q 497-D \\
G W-2046-Q 397-D \\
G W-2046-Q 497-A \\
G W-2046-Q 497-B \\
G W-2046-Q 497-C \\
G W-2046-Q 497-D \\
G W-2046-0397-D \\
G W-2046-Q 497-A\end{array}$ & $\begin{array}{l}07 / 11 / 97 \\
10 / 02 / 97 \\
10 / 07 / 97 \\
10 / 08 / 97 \\
10 / 09 / 97 \\
07 / 11 / 97 \\
10 / 02 / 97 \\
10 / 07 / 97 \\
10 / 08 / 97 \\
10 / 09 / 97 \\
07 / 11 / 97 \\
10 / 02 / 97 \\
10 / 07 / 97 \\
10 / 08 / 97 \\
10 / 09 / 97 \\
07 / 11 / 97 \\
10 / 02 / 97 \\
10 / 07 / 97 \\
10 / 08 / 97 \\
10 / 09 / 97 \\
07 / 11 / 97 \\
10 / 02 / 97 \\
10 / 07 / 97 \\
10 / 08 / 97 \\
10 / 09 / 97 \\
07 / 11 / 97 \\
10 / 02 / 97 \\
10 / 07 / 97 \\
10 / 08 / 97 \\
10 / 09 / 97 \\
07 / 11 / 97 \\
10 / 02 / 97 \\
10 / 07 / 97 \\
10 / 08 / 97 \\
10 / 09 / 97 \\
07 / 11 / 97 \\
10 / 02 / 97 \\
10 / 07 / 97 \\
10 / 08 / 97 \\
10 / 09 / 97 \\
07 / 11 / 97 \\
10 / 02 / 97 \\
10 / 07 / 97 \\
10 / 08 / 97 \\
10 / 09 / 97 \\
10 / 02 / 97 \\
10 / 07 / 97 \\
10 / 08 / 97 \\
10 / 09 / 97 \\
07 / 11 / 97 \\
10 / 02 / 97 \\
10 / 07 / 97 \\
10 / 08 / 97 \\
10 / 09 / 97 \\
07 / 11 / 97 \\
10 / 02 / 97 \\
10 / 07 / 97 \\
10 / 08 / 97 \\
10 / 09 / 97 \\
07 / 11 / 97 \\
10 / 02 / 97\end{array}$ & 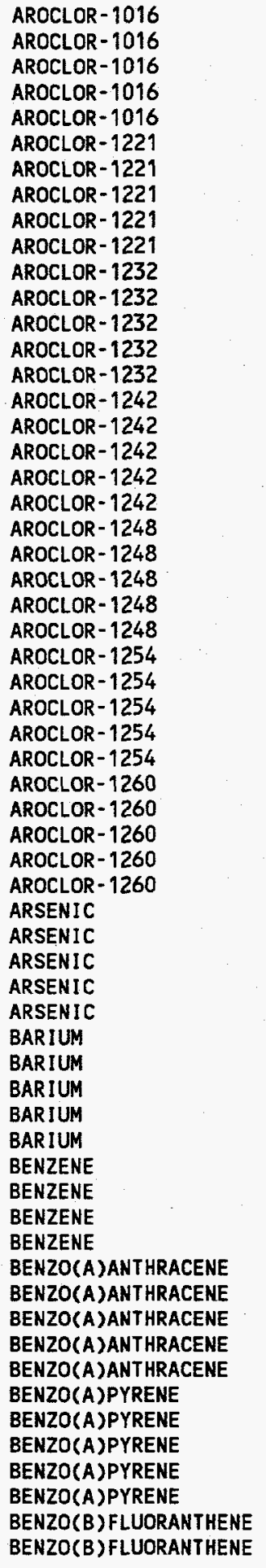 & $\begin{array}{l}\text { ND } \\
\text { ND } \\
\text { ND } \\
\text { ND } \\
\text { ND } \\
\text { ND } \\
\text { ND } \\
\text { ND } \\
\text { ND } \\
\text { ND } \\
\text { ND } \\
\text { ND } \\
\text { ND } \\
\text { ND } \\
\text { ND } \\
\text { ND } \\
\text { ND } \\
\text { ND } \\
\text { ND } \\
\text { ND } \\
\text { ND } \\
\text { ND } \\
\text { ND } \\
\text { ND } \\
\text { ND } \\
\text { ND } \\
\text { ND } \\
\text { ND } \\
\text { ND } \\
\text { ND } \\
\text { ND } \\
\text { ND } \\
\text { ND } \\
\text { ND } \\
\text { ND } \\
\text { ND } \\
\text { ND } \\
\text { ND } \\
\text { ND } \\
\text { ND } \\
157 \\
\text { 228 } \\
194 \\
167 \\
\text { 214 } \\
\text { ND } \\
\text { ND } \\
\text { ND } \\
\text { ND } \\
\text { ND } \\
\text { ND } \\
\text { ND } \\
\text { ND } \\
\text { ND } \\
\text { ND } \\
\text { ND } \\
\text { ND } \\
\text { ND } \\
\text { ND } \\
\text { ND } \\
\text { ND }\end{array}$ & $\begin{array}{l}1.0 \\
1.0 \\
1.0 \\
1.0 \\
1.0 \\
1.0 \\
1.0 \\
1.0 \\
1.0 \\
1.0 \\
1.0 \\
1.0 \\
1.0 \\
1.0 \\
1.0 \\
1.0 \\
1.0 \\
1.0 \\
1.0 \\
1.0 \\
1.0 \\
1.0 \\
1.0 \\
1.0 \\
1.0 \\
1.0 \\
1.0 \\
1.0 \\
1.0 \\
1.0 \\
1.0 \\
1.0 \\
1.0 \\
1.0 \\
1.0 \\
1.7 \\
4.0 \\
4.0 \\
4.0 \\
4.0 \\
0.60 \\
2.0 \\
2.0 \\
2.0 \\
2.0 \\
10 \\
10 \\
10 \\
10 \\
5.0 \\
0.5 \\
0.5 \\
0.5 \\
0.5 \\
5.0 \\
0.5 \\
0.5 \\
0.5 \\
0.5 \\
0.0 \\
0.5\end{array}$ & 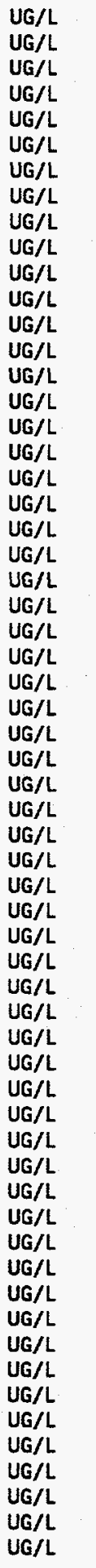 & $\begin{array}{l}\text { EPA } 8080 A \\
\text { EPA } 8081 \\
\text { EPA } 8081 \\
\text { EPA } 8081 \\
\text { EPA } 8081 \\
\text { EPA } 8080 A \\
\text { EPA } 8081 \\
\text { EPA } 8081 \\
\text { EPA } 8081 \\
\text { EPA } 8081 \\
\text { EPA } 8080 A \\
\text { EPA } 8081 \\
\text { EPA } 8081 \\
\text { EPA } 8081 \\
\text { EPA } 8081 \\
\text { EPA } 8080 A \\
\text { EPA } 8081 \\
\text { EPA } 8081 \\
\text { EPA } 8081 \\
\text { EPA } 8081 \\
\text { EPA } 8080 A \\
\text { EPA } 8081 \\
\text { EPA } 8081 \\
\text { EPA } 8081 \\
\text { EPA } 8081 \\
\text { EPA } 8080 A \\
\text { EPA } 8081 \\
\text { EPA } 8081 \\
\text { EPA } 8081 \\
\text { EPA } 8081 \\
\text { EPA } 8080 A \\
\text { EPA } 8081 \\
\text { EPA } 8081 \\
\text { EPA } 8081 \\
\text { EPA } 8081 \\
\text { EPA } C L P \\
\text { EPA } 6010 A \\
\text { EPA } 6010 A \\
\text { EPA } 6010 A \\
\text { EPA } 6010 A \\
\text { EPA } C L P \\
\text { EPA } 6010 A \\
\text { EPA } 6010 A \\
\text { EPA } 6010 A \\
\text { EPA } 6010 A \\
\text { EPA } C L P \\
\text { EPA } C L P \\
\text { EPA } C 1 P \\
\text { EPA CLP } \\
\text { EPA } 8310 \\
\text { EPA } 8310 \\
\text { EPA } 8310 \\
\text { SH846 } 8310 \\
\text { SW846 } 8310 \\
\text { EPA } 8310 \\
\text { EPA } 8310 \\
\text { EPA } 8310 \\
\text { SH846 } 8310 \\
\text { SW846 } 8310 \\
\text { EPA } 8310 \\
\text { EPA } 8310\end{array}$ \\
\hline
\end{tabular}




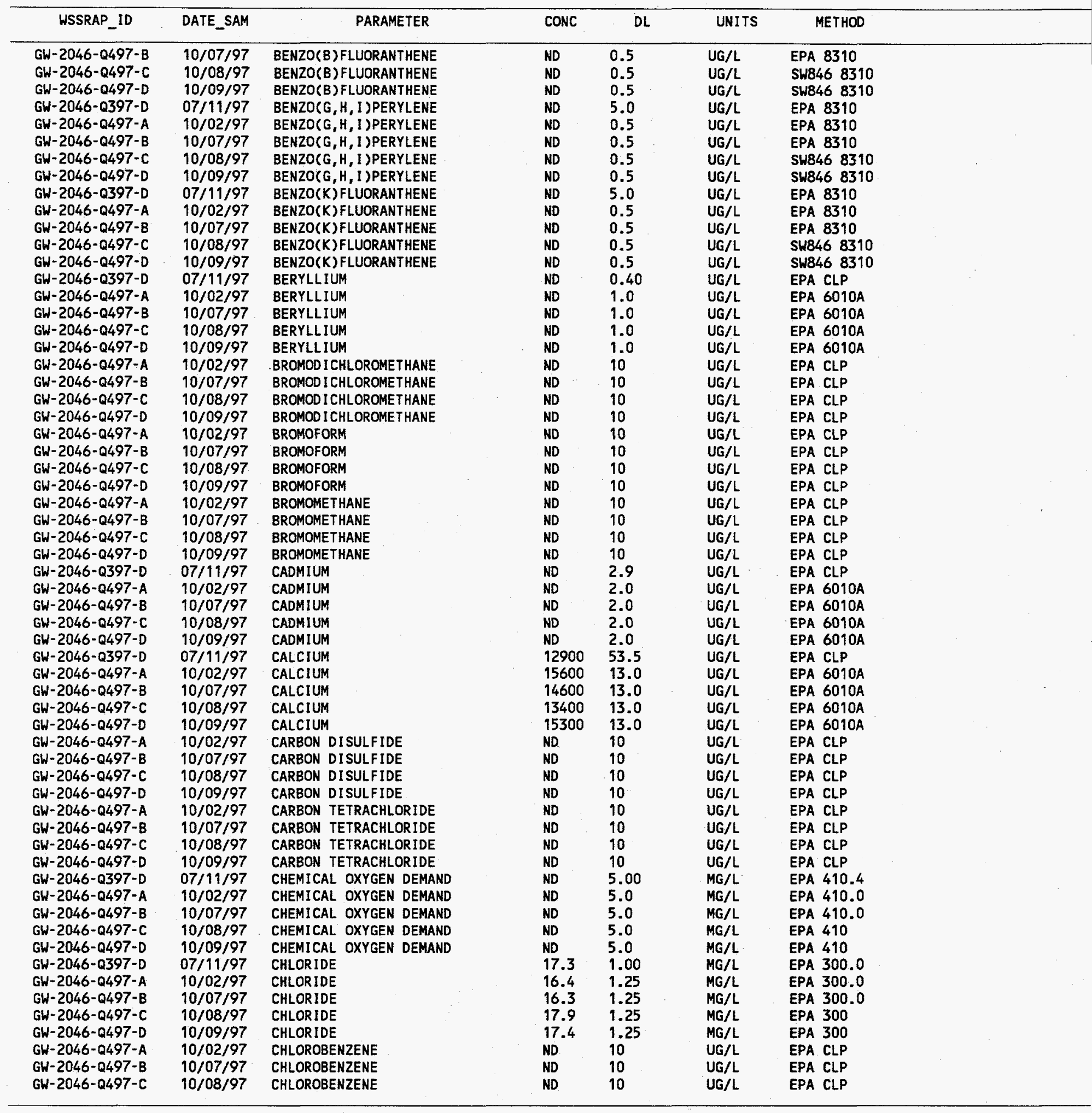




\begin{tabular}{|c|c|c|c|c|c|c|}
\hline WSSRAP_ID & DATE_SAM & PARAMETER & CONC & DL & UNITS & METHOO \\
\hline 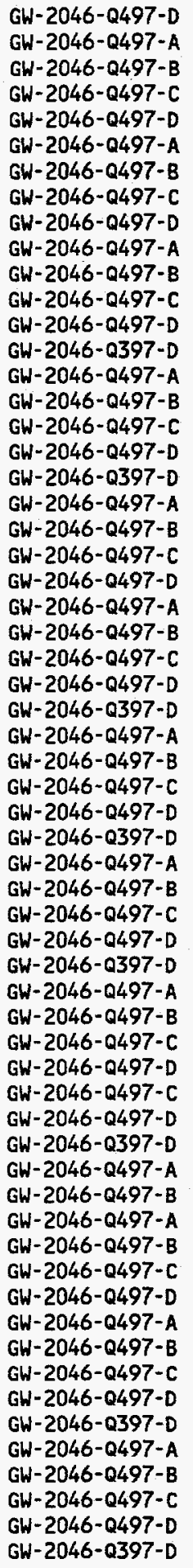 & $\begin{array}{l}10 / 09 / 97 \\
10 / 02 / 97 \\
10 / 07 / 97 \\
10 / 08 / 97 \\
10 / 09 / 97 \\
10 / 02 / 97 \\
10 / 07 / 97 \\
10 / 08 / 97 \\
10 / 09 / 97 \\
10 / 02 / 97 \\
10 / 07 / 97 \\
10 / 08 / 97 \\
10 / 09 / 97 \\
07 / 11 / 97 \\
10 / 02 / 97 \\
10 / 07 / 97 \\
10 / 08 / 97 \\
10 / 09 / 97 \\
07 / 11 / 97 \\
10 / 02 / 97 \\
10 / 07 / 97 \\
10 / 08 / 97 \\
10 / 09 / 97 \\
10 / 02 / 97 \\
10 / 07 / 97 \\
10 / 08 / 97 \\
10 / 09 / 97 \\
07 / 11 / 97 \\
10 / 02 / 97 \\
10 / 07 / 97 \\
10 / 08 / 97 \\
10 / 09 / 97 \\
07 / 11 / 97 \\
10 / 02 / 97 \\
10 / 07 / 97 \\
10 / 08 / 97 \\
10 / 09 / 97 \\
07 / 11 / 97 \\
10 / 02 / 97 \\
10 / 07 / 97 \\
10 / 08 / 97 \\
10 / 09 / 97 \\
10 / 08 / 97 \\
10 / 09 / 97 \\
07 / 11 / 97 \\
10 / 02 / 97 \\
10 / 07 / 97 \\
10 / 02 / 97 \\
10 / 07 / 97 \\
10 / 08 / 97 \\
10 / 09 / 97 \\
10 / 02 / 97 \\
10 / 07 / 97 \\
10 / 08 / 97 \\
10 / 09 / 97 \\
07 / 11 / 97 \\
10 / 02 / 97 \\
10 / 07 / 97 \\
10 / 08 / 97 \\
10 / 09 / 97 \\
07 / 11 / 97\end{array}$ & 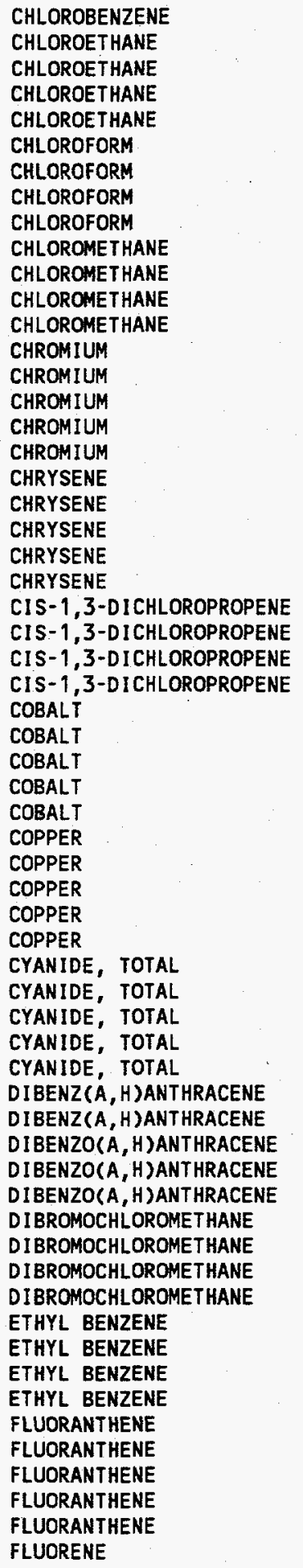 & $\begin{array}{l}\text { ND } \\
\text { ND } \\
\text { ND } \\
\text { ND } \\
\text { ND } \\
\text { ND } \\
\text { ND } \\
\text { ND } \\
\text { ND } \\
\text { ND } \\
\text { ND } \\
2.3 \\
2.3 \\
3.6 \\
\text { ND } \\
\text { ND } \\
\text { ND } \\
\text { ND } \\
\text { ND } \\
\text { ND } \\
\text { ND } \\
\text { ND } \\
\text { ND } \\
\text { ND } \\
\text { ND } \\
\text { ND } \\
\text { ND } \\
\text { ND } \\
\text { ND } \\
\text { ND } \\
\text { ND } \\
\text { ND } \\
\text { ND } \\
\text { ND } \\
\text { ND } \\
\text { ND } \\
\text { ND } \\
\text { ND } \\
\text { ND }\end{array}$ & $\begin{array}{l}10 \\
10 \\
10 \\
10 \\
10 \\
10 \\
10 \\
10 \\
10 \\
10 \\
10 \\
10 \\
10 \\
2.7 \\
3.7 \\
2.0 \\
2.0 \\
2.0 \\
5.0 \\
0.5 \\
0.5 \\
0.5 \\
0.5 \\
10 \\
10 \\
10 \\
10 \\
4.0 \\
2.0 \\
2.0 \\
2.0 \\
2.0 \\
4.4 \\
2.0 \\
2.0 \\
2.0 \\
2.0 \\
1.50 \\
4.0 \\
4.0 \\
4.0 \\
4.0 \\
0.5 \\
0.5 \\
5.0 \\
0.5 \\
0.5 \\
10 \\
10 \\
10 \\
10 \\
10 \\
10 \\
10 \\
10 \\
5.0 \\
0.5 \\
0.5 \\
0.5 \\
0.5 \\
5.0\end{array}$ & $\begin{array}{l}U G / L \\
U G / L \\
U G / L \\
U G / L \\
U G / L \\
U G / L \\
U G / L \\
U G / L \\
U G / L \\
U G / L \\
U G / L \\
U G / L \\
U G / L \\
U G / L \\
U G / L \\
U G / L \\
U G / L \\
U G / L \\
U G / L \\
U G / L \\
U G / L \\
U G / L \\
U G / L \\
U G / L \\
U G / L \\
U G / L \\
U G / L \\
U G / L \\
U G / L \\
U G / L \\
U G / L \\
U G / L \\
U G / L \\
U G / L \\
U G / L \\
U G / L \\
U G / L \\
U G / L \\
U G / L \\
U G / L \\
U G / L \\
U G / L \\
U G / L \\
U G / L \\
U G / L \\
U G / L \\
U G / L \\
U G / L \\
U G / L \\
U G / L \\
U G / L \\
U G / L \\
U G / L \\
U G / L \\
U G / L \\
U G / L \\
U G / L \\
U G / L \\
U G / L \\
U G / L \\
U G / L \\
U\end{array}$ & $\begin{array}{l}\text { EPA CLP } \\
\text { EPA CLP } \\
\text { EPA CLP } \\
\text { EPA CLP } \\
\text { EPA CLP } \\
\text { EPA CLP } \\
\text { EPA CLP } \\
\text { EPA CLP } \\
\text { EPA CLP } \\
\text { EPA CLP } \\
\text { EPA CLP } \\
\text { EPA CLP } \\
\text { EPA CLP } \\
\text { EPA CLP } \\
\text { EPA } 6010 A \\
\text { EPA } 6010 A \\
\text { EPA } 6010 A \\
\text { EPA } 6010 A \\
\text { EPA } 8310 \\
\text { EPA } 8310 \\
\text { EPA } 8310 \\
\text { SH846 } 8310 \\
\text { SW846 } 8310 \\
\text { EPA CLP } \\
\text { EPA CLP } \\
\text { EPA CLP } \\
\text { EPA CLP } \\
\text { EPA CLP } \\
\text { EPA } 6010 A \\
\text { EPA } 6010 A \\
\text { EPA } 6010 A \\
\text { EPA } 6010 A \\
\text { EPA CLP } \\
\text { EPA } 6010 A \\
\text { EPA } 6010 A \\
\text { EPA } 6010 A \\
\text { EPA } 6010 A \\
\text { EPA CLP } \\
\text { EPA CLP } \\
\text { EPA CLP } \\
\text { EPA CLP } \\
\text { EPA CLP } \\
\text { SH846 } 8310 \\
\text { SW846 } 8310 \\
\text { EPA } 8310 \\
\text { EPA } 8310 \\
\text { EPA } 8310 \\
\text { EPA CLP } \\
\text { EPA CLP } \\
\text { EPA CLP } \\
\text { EPA CLP } \\
\text { EPA CLP } \\
\text { EPA CLP } \\
\text { EPA CLP } \\
\text { EPA CLP } \\
\text { EPA } 8310 \\
\text { EPA } 8310 \\
\text { EPA } 8310 \\
\text { SW846 } 8310 \\
\text { SH846 } 8310 \\
\text { EPA } 8310\end{array}$ \\
\hline
\end{tabular}




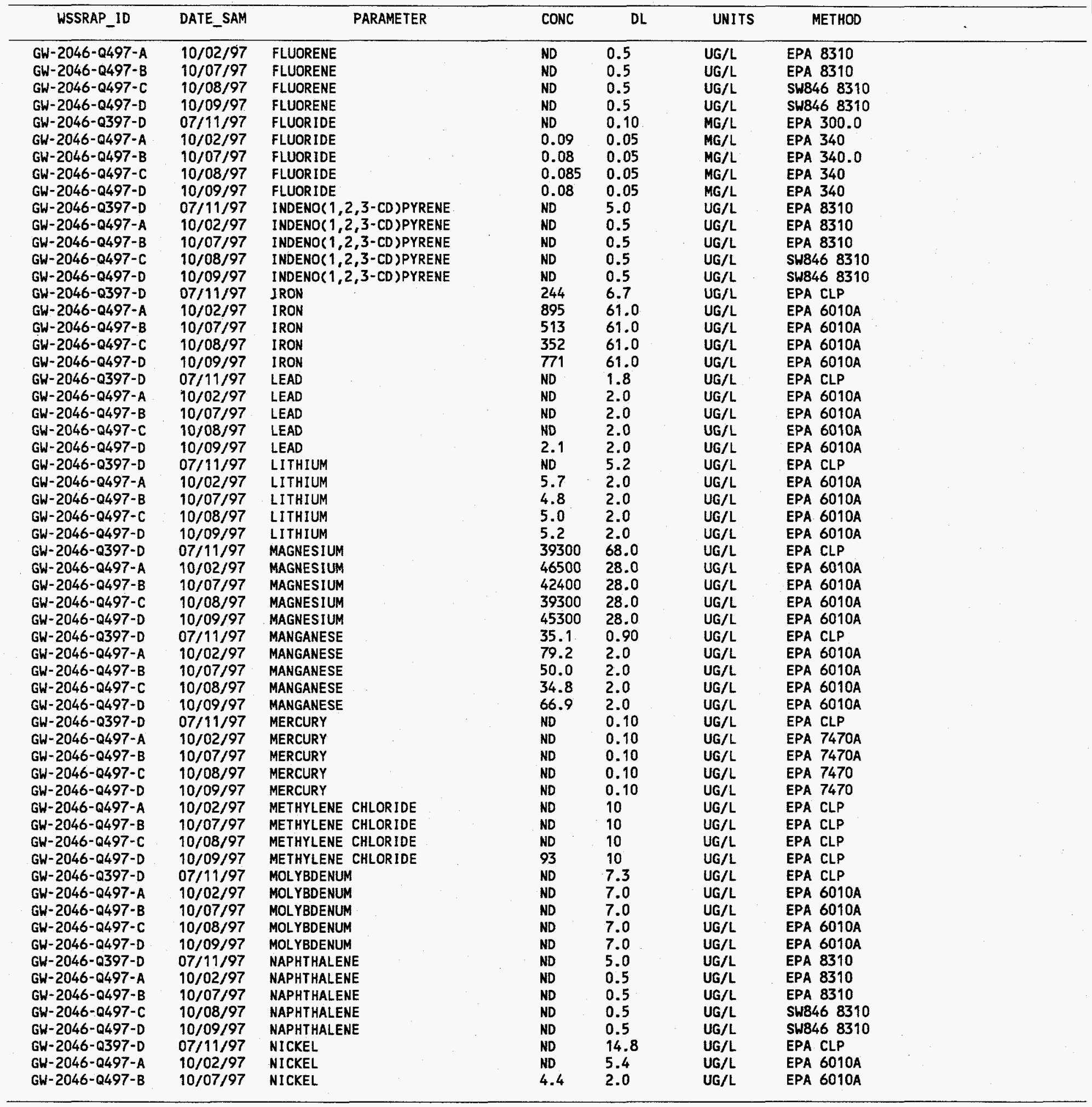




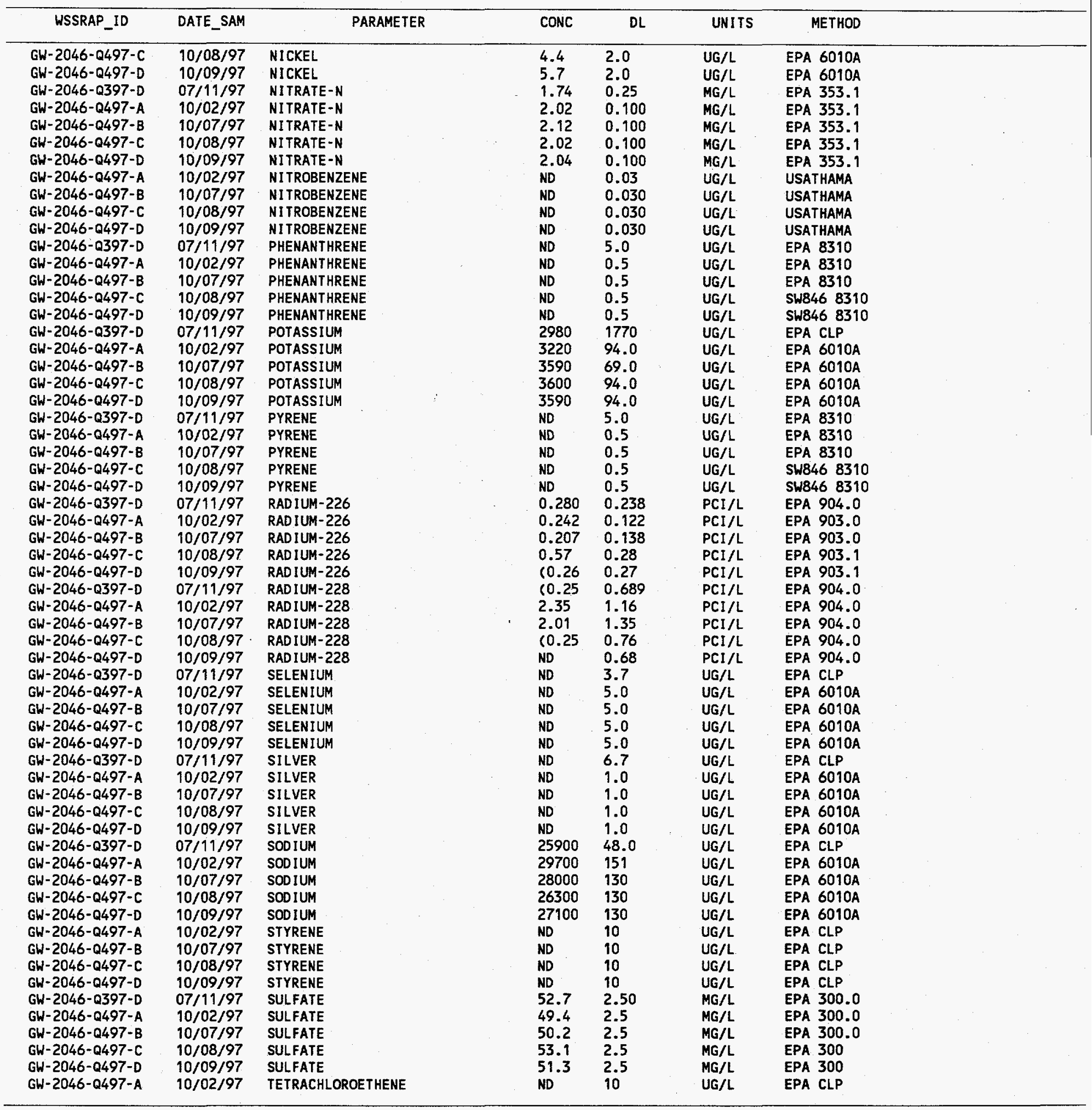




\begin{tabular}{|c|c|c|c|c|c|c|}
\hline WSSRAP_ID & DATE_SAM & PARAMETER & CONC & $\mathrm{DL}$ & UNITS & METHOD \\
\hline 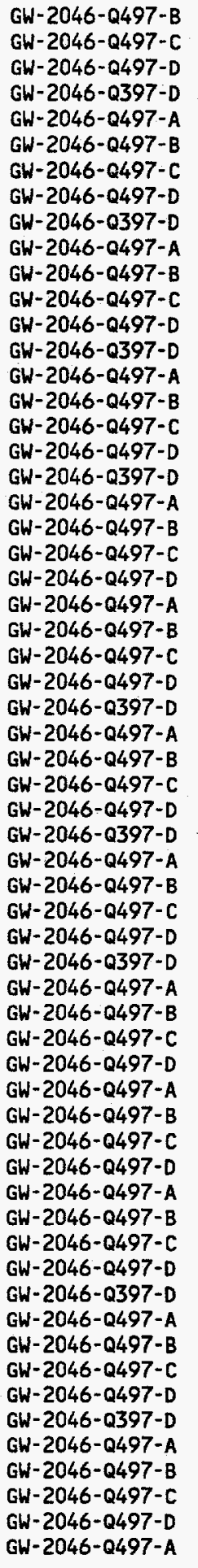 & $\begin{array}{l}10 / 07 / 97 \\
10 / 08 / 97 \\
10 / 09 / 97 \\
07 / 11 / 97 \\
10 / 02 / 97 \\
10 / 07 / 97 \\
10 / 08 / 97 \\
10 / 09 / 97 \\
07 / 11 / 97 \\
10 / 02 / 97 \\
10 / 07 / 97 \\
10 / 08 / 97 \\
10 / 09 / 97 \\
07 / 11 / 97 \\
10 / 02 / 97 \\
10 / 07 / 97 \\
10 / 08 / 97 \\
10 / 09 / 97 \\
07 / 11 / 97 \\
10 / 02 / 97 \\
10 / 07 / 97 \\
10 / 08 / 97 \\
10 / 09 / 97 \\
10 / 02 / 97 \\
10 / 07 / 97 \\
10 / 08 / 97 \\
10 / 09 / 97 \\
07 / 11 / 97 \\
10 / 02 / 97 \\
10 / 07 / 97 \\
10 / 08 / 97 \\
10 / 09 / 97 \\
07 / 11 / 97 \\
10 / 02 / 97 \\
10 / 07 / 97 \\
10 / 08 / 97 \\
10 / 09 / 97 \\
07 / 11 / 97 \\
10 / 02 / 97 \\
10 / 07 / 97 \\
10 / 08 / 97 \\
10 / 09 / 97 \\
10 / 02 / 97 \\
10 / 07 / 97 \\
10 / 08 / 97 \\
10 / 09 / 97 \\
10 / 02 / 97 \\
10 / 07 / 97 \\
10 / 08 / 97 \\
10 / 09 / 97 \\
07 / 11 / 97 \\
10 / 02 / 97 \\
10 / 07 / 97 \\
10 / 08 / 97 \\
10 / 09 / 97 \\
07 / 11 / 97 \\
10 / 02 / 97 \\
10 / 07 / 97 \\
10 / 08 / 97 \\
10 / 09 / 97 \\
10 / 02 / 97\end{array}$ & $\begin{array}{l}\text { TETRACHLOROETHENE } \\
\text { TETRACHLOROETHENE } \\
\text { TETRACHLOROETHENE } \\
\text { THALLIUM } \\
\text { THALLIUM } \\
\text { THALLIUM } \\
\text { THALLIUM } \\
\text { THALLIUM } \\
\text { THORIUM-228 } \\
\text { THORIUM-228 } \\
\text { THORIUM-228 } \\
\text { THORIUM-228 } \\
\text { THORIUM-228 } \\
\text { THORIUM-230 } \\
\text { THORIUM-230 } \\
\text { THORIUM-230 } \\
\text { THORIUM-230 } \\
\text { THORIUM-230 } \\
\text { THORIUM-232 } \\
\text { THORIUM-232 } \\
\text { THORIUM-232 } \\
\text { THORIUM-232 } \\
\text { THORIUM-232 } \\
\text { TOLUENE } \\
\text { TOLUENE } \\
\text { TOLUENE } \\
\text { TOLUENE } \\
\text { TOTAL DISSOLVED SOLIDS } \\
\text { TOTAL DISSOLVED SOLIDS } \\
\text { TOTAL DISSOLVED SOLIDS } \\
\text { TOTAL DISSOLVED SOLIDS } \\
\text { TOTAL DISSOLVED SOLIDS } \\
\text { TOTAL ORGANIC CARBON } \\
\text { TOTAL ORGANIC CARBON } \\
\text { TOTAL ORGANIC CARBON } \\
\text { TOTAL ORGANIC CARBON } \\
\text { TOTAL ORGANIC CARBON } \\
\text { TOX } \\
\text { TOX } \\
\text { TOX } \\
\text { TOX } \\
\text { TOX } \\
\text { TRANS-1,3-DICHLOROPROPENE } \\
\text { TRANS-1,3-DICHLOROPROPENE } \\
\text { TRANS-1,3-DICHLOROPROPENE } \\
\text { TRANS-1,3-DICHLOROPROPENE } \\
\text { TRICHLOROETHENE } \\
\text { TRICHLOROETHENE } \\
\text { TRICHLOROETHENE } \\
\text { TRICHLOROETHENE } \\
\text { URANIUM, TOTAL } \\
\text { URANIUM, TOTAL } \\
\text { URANIUM, TOTAL } \\
\text { URANIUM, TOTAL } \\
\text { URANIUM, TOTAL } \\
\text { VANADIUM } \\
\text { VANADIUM } \\
\text { VANADIUM } \\
\text { VANADIUM } \\
\text { VANADIUM } \\
\text { VINYL CHLORIDE } \\
\text { THE }\end{array}$ & $\begin{array}{l}\text { ND } \\
\text { ND } \\
\text { ND } \\
\text { ND } \\
\text { ND } \\
\text { ND } \\
\text { ND } \\
\text { ND } \\
\text { C.00 } \\
C 0.09 \\
C 0.10 \\
60.14 \\
\text { ND } \\
0.126 \\
C 0.09 \\
C 0.21 \\
C 0.00 \\
\text { ND } \\
C 0.00 \\
C 0.09 \\
\text { ND } \\
\text { ND } \\
\text { ND } \\
\text { ND } \\
\text { ND } \\
\text { ND } \\
\text { ND } \\
529 \\
684 \\
538 \\
526 \\
500 \\
1.51 \\
31.5 \\
42.4 \\
45.5 \\
42.0 \\
0.017 \\
0.026 \\
0.014 \\
0.025 \\
0.014 \\
\text { ND } \\
\text { ND } \\
\text { ND } \\
\text { ND } \\
\text { ND } \\
\text { ND } \\
\text { ND } \\
\text { ND } \\
0.981 \\
1.04 \\
0.942 \\
0.863 \\
0.873 \\
10.0 \\
\text { ND } \\
\text { ND } \\
\text { ND } \\
2.7 \\
\text { ND }\end{array}$ & 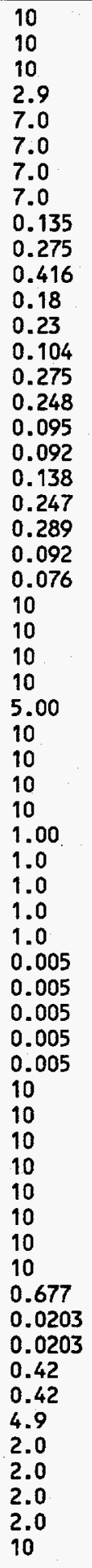 & $\begin{array}{l}\text { UG/L } \\
U G / L \\
U G / L \\
U G / L \\
U G / L \\
U G / L \\
U G / L \\
U G / L \\
P C I / L \\
P C I / L \\
P C I / L \\
P C I / L \\
P C I / L \\
P C I / L \\
P C I / L \\
P C I / L \\
P C I / L \\
P C I / L \\
P C I / L \\
P C I / L \\
P C I / L \\
P C I / L \\
P C I / L \\
U G / L \\
U G / L \\
U G / L \\
U G / L \\
M G / L \\
M G / L \\
M G / L \\
M G / L \\
M G / L \\
M G / L \\
M G / L \\
M G / L \\
M G / L \\
M G / L \\
P C I / L \\
U G / L \\
U G / L \\
U G / L \\
U G / L \\
U G / L \\
U G / L \\
M G / L \\
M G / L \\
M G / L \\
M G / L \\
U G / L \\
U G / L \\
U G / L \\
U G / L \\
U G / L \\
U G / L \\
U G / L \\
\end{array}$ & $\begin{array}{l}\text { EPA CLP } \\
\text { EPA CLP } \\
\text { EPA CLP } \\
\text { EPA CLP } \\
\text { EPA } 6010 A \\
\text { EPA } 6010 A \\
\text { EPA } 6010 A \\
\text { EPA } 6010 A \\
\text { NAS-NS-3004 } \\
\text { EML TH-01 } \\
\text { EML TH-01 } \\
\text { EPA } 907.0 \\
\text { EPA } 907.0 \\
\text { NAS-NS-3004 } \\
\text { EML TH-01 } \\
\text { EML TH-01 } \\
\text { EPA } 907.0 \\
\text { EPA } 907.0 \\
\text { NAS-NS-3004 } \\
\text { EML TH-01 } \\
\text { EML TH-01 } \\
\text { EPA } 907.0 \\
\text { EPA } 907.0 \\
\text { EPA CLP } \\
\text { EPA CLP } \\
\text { EPA CLP } \\
\text { EPA CLP } \\
\text { EPA } 160.1 \\
\text { EPA } 160.1 \\
\text { EPA } 160.1 \\
\text { EPA } 160.1 \\
\text { EPA } 160.1 \\
\text { EPA } 415.1 \\
\text { EPA } 415.1 \\
\text { EPA } 415.1 \\
\text { EPA } 415.1 \\
\text { EPA } 415.1 \\
\text { EPA } 9020 \\
\text { EPA } 9020 A \\
\text { EPA } 9020 A \\
\text { EPA } 9020 A \\
\text { EPA } 9020 A \\
\text { EPA CLP } \\
\text { EPA CLP } \\
\text { EPA CLP } \\
\text { EPA CLP } \\
\text { EPA CLP } \\
\text { EPA CLP } \\
\text { EPA CLP } \\
\text { EPA CLP } \\
\text { ASTM } 5174-91 \\
\text { ASTM } 5174-91 \\
\text { ASTM } 5174-91 \\
\text { ASTM } 5174-91 \\
\text { ASTM } 5174-91 \\
\text { EPA CLP } \\
\text { EPA } 6010 A \\
\text { EPA } 6010 A \\
\text { EPA } 6010 A \\
\text { EPA } 6010 A \\
\text { EPA CLP } \\
\text { EPA }\end{array}$ \\
\hline
\end{tabular}




\begin{tabular}{|c|c|c|c|c|c|c|}
\hline WSSRAP_ID & DATE_SAM & PARAMETER & CONC & $D L$ & UNITS & METHOD \\
\hline 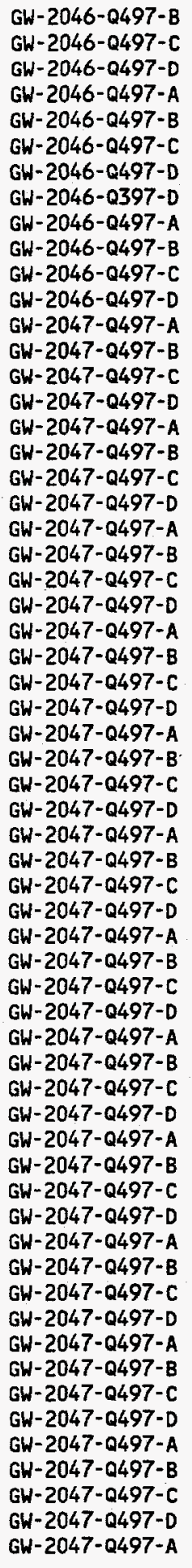 & $\begin{array}{l}10 / 07 / 97 \\
10 / 08 / 97 \\
10 / 09 / 97 \\
10 / 02 / 97 \\
10 / 07 / 97 \\
10 / 08 / 97 \\
10 / 09 / 97 \\
07 / 11 / 97 \\
10 / 02 / 97 \\
10 / 07 / 97 \\
10 / 08 / 97 \\
10 / 09 / 97 \\
10 / 02 / 97 \\
10 / 07 / 97 \\
10 / 08 / 97 \\
10 / 09 / 97 \\
10 / 02 / 97 \\
10 / 07 / 97 \\
10 / 08 / 97 \\
10 / 09 / 97 \\
10 / 02 / 97 \\
10 / 07 / 97 \\
10 / 08 / 97 \\
10 / 09 / 97 \\
10 / 02 / 97 \\
10 / 07 / 97 \\
10 / 08 / 97 \\
10 / 09 / 97 \\
10 / 02 / 97 \\
10 / 07 / 97 \\
10 / 08 / 97 \\
10 / 09 / 97 \\
10 / 02 / 97 \\
10 / 07 / 97 \\
10 / 08 / 97 \\
10 / 09 / 97 \\
10 / 02 / 97 \\
10 / 07 / 97 \\
10 / 08 / 97 \\
10 / 09 / 97 \\
10 / 02 / 97 \\
10 / 07 / 97 \\
10 / 08 / 97 \\
10 / 09 / 97 \\
10 / 02 / 97 \\
10 / 07 / 97 \\
10 / 08 / 97 \\
10 / 09 / 97 \\
10 / 02 / 97 \\
10 / 07 / 97 \\
10 / 08 / 97 \\
10 / 09 / 97 \\
10 / 02 / 97 \\
10 / 07 / 97 \\
10 / 08 / 97 \\
10 / 09 / 97 \\
10 / 02 / 97 \\
10 / 07 / 97 \\
10 / 08 / 97 \\
10 / 09 / 97 \\
10 / 02 / 97\end{array}$ & 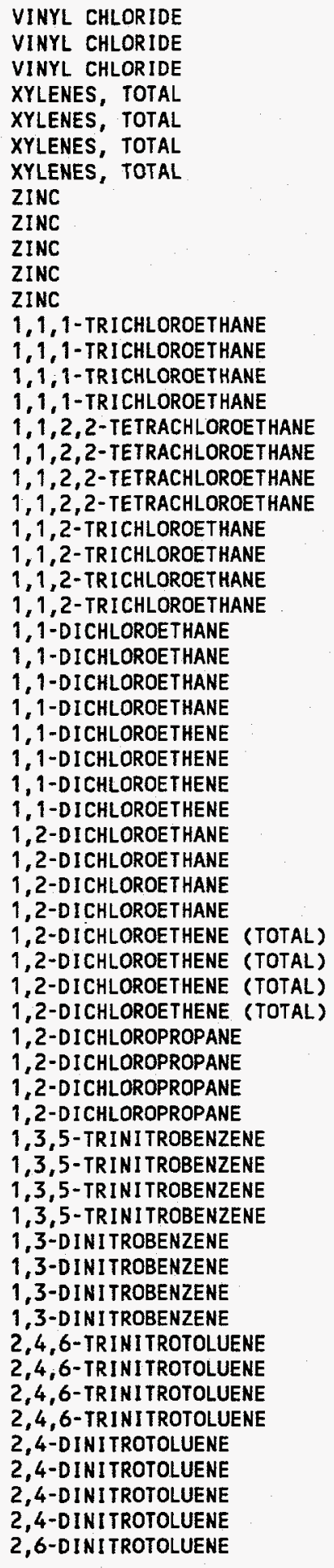 & 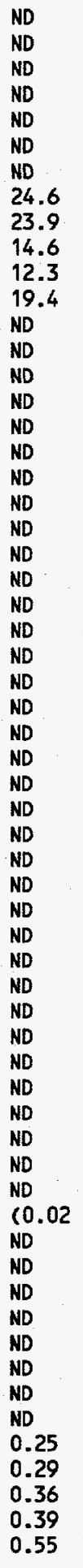 & $\begin{array}{l}10 \\
10 \\
10 \\
10 \\
10 \\
10 \\
10 \\
4.8 \\
6.0 \\
6.0 \\
6.0 \\
6.0 \\
10 \\
10 \\
10 \\
10 \\
10 \\
10 \\
10 \\
10 \\
10 \\
10 \\
10 \\
10 \\
10 \\
10 \\
10 \\
10 \\
10 \\
10 \\
10 \\
10 \\
10 \\
10 \\
10 \\
10 \\
10 \\
10 \\
10 \\
10 \\
10 \\
10 \\
10 \\
10 \\
0.03 \\
0.030 \\
0.030 \\
0.030 \\
0.09 \\
0.090 \\
0.090 \\
0.090 \\
0.03 \\
0.030 \\
0.030 \\
0.030 \\
0.03 \\
0.030 \\
0.030 \\
0.030 \\
0.010\end{array}$ & 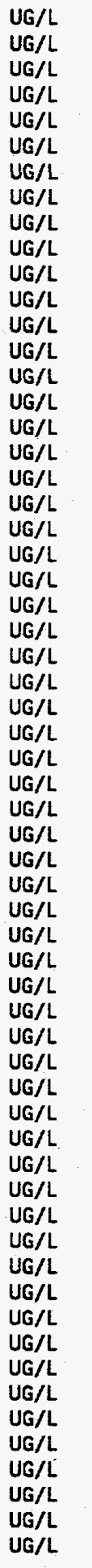 & $\begin{array}{l}\text { EPA CLP } \\
\text { EPA CLP } \\
\text { EPA CLP } \\
\text { EPA CLP } \\
\text { EPA CLP } \\
\text { EPA CLP } \\
\text { EPA CLP } \\
\text { EPA CLP } \\
\text { EPA } 6010 A \\
\text { EPA } 6010 A \\
\text { EPA } 6010 A \\
\text { EPA } 6010 A \\
\text { EPA CLP } \\
\text { EPA CLP } \\
\text { EPA CLP } \\
\text { EPA CLP } \\
\text { EPA CLP } \\
\text { EPA CLP } \\
\text { EPA CLP } \\
\text { EPA CLP } \\
\text { EPA CLP } \\
\text { EPA CLP } \\
\text { EPA CLP } \\
\text { EPA CLP } \\
\text { EPA CLP } \\
\text { EPA CLP } \\
\text { EPA CLP } \\
\text { EPA CLP } \\
\text { EPA CLP } \\
\text { EPA CLP } \\
\text { EPA CLP } \\
\text { EPA CLP } \\
\text { EPA CLP } \\
\text { EPA CLP } \\
\text { EPA CLP } \\
\text { EPA CLP } \\
\text { EPA CLP } \\
\text { EPA CLP } \\
\text { EPA CLP } \\
\text { EPA CLP } \\
\text { EPA CLP } \\
\text { EPA CLP } \\
\text { EPA CLP } \\
\text { EPA CLP } \\
\text { USATHAMA } \\
\text { USATHAMA } \\
\text { USATHAMA } \\
\text { USATHAMA } \\
\text { USATHAMA } \\
\text { USATHAMA } \\
\text { USATHAMA } \\
\text { USATHAMA } \\
\text { USATHAMA } \\
\text { USATHAMA } \\
\text { USATHAMA } \\
\text { USATHAMA } \\
\text { USATHAMA } \\
\text { USATHAMA } \\
\text { USATHAMA } \\
\text { USATHAMA } \\
\text { USATHAMA }\end{array}$ \\
\hline
\end{tabular}




\begin{tabular}{|c|c|c|c|c|c|c|}
\hline WSSRAP_ID & DATE_SAM & PARAMETER & CONC & $\mathrm{DL}$ & UNITS & METHOD \\
\hline 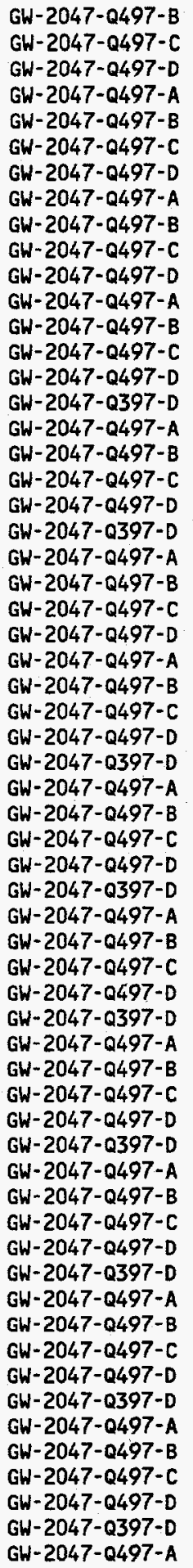 & $\begin{array}{l}10 / 07 / 97 \\
10 / 08 / 97 \\
10 / 09 / 97 \\
10 / 02 / 97 \\
10 / 07 / 97 \\
10 / 08 / 97 \\
10 / 09 / 97 \\
10 / 02 / 97 \\
10 / 07 / 97 \\
10 / 08 / 97 \\
10 / 09 / 97 \\
10 / 02 / 97 \\
10 / 07 / 97 \\
10 / 08 / 97 \\
10 / 09 / 97 \\
07 / 11 / 97 \\
10 / 02 / 97 \\
10 / 07 / 97 \\
10 / 08 / 97 \\
10 / 09 / 97 \\
07 / 11 / 97 \\
10 / 02 / 97 \\
10 / 07 / 97 \\
10 / 08 / 97 \\
10 / 09 / 97 \\
10 / 02 / 97 \\
10 / 07 / 97 \\
10 / 08 / 97 \\
10 / 09 / 97 \\
07 / 11 / 97 \\
10 / 02 / 97 \\
10 / 07 / 97 \\
10 / 08 / 97 \\
10 / 09 / 97 \\
07 / 11 / 97 \\
10 / 02 / 97 \\
10 / 07 / 97 \\
10 / 08 / 97 \\
10 / 09 / 97 \\
07 / 11 / 97 \\
10 / 02 / 97 \\
10 / 07 / 97 \\
10 / 08 / 97 \\
10 / 09 / 97 \\
07 / 11 / 97 \\
10 / 02 / 97 \\
10 / 07 / 97 \\
10 / 08 / 97 \\
10 / 09 / 97 \\
07 / 11 / 97 \\
10 / 02 / 97 \\
10 / 07 / 97 \\
10 / 08 / 97 \\
10 / 09 / 97 \\
07 / 11 / 97 \\
10 / 02 / 97 \\
10 / 07 / 97 \\
10 / 08 / 97 \\
10 / 09 / 97 \\
07 / 11 / 97 \\
10 / 02 / 97\end{array}$ & 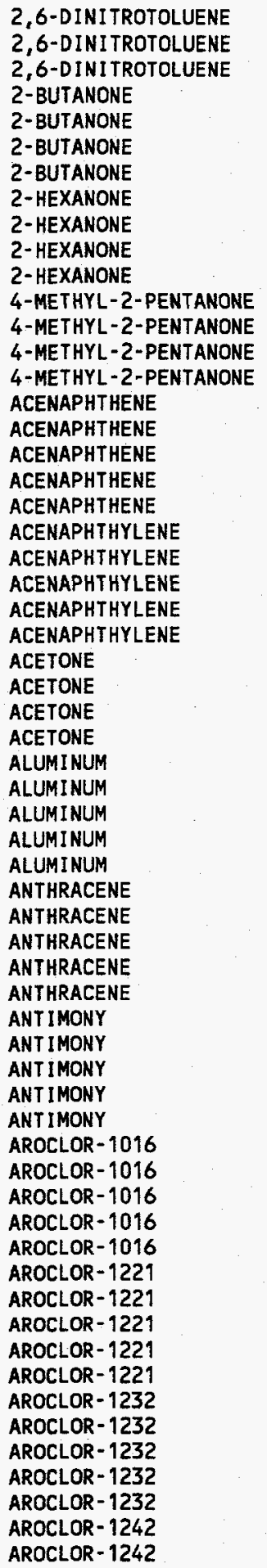 & $\begin{array}{l}0.61 \\
0.75 \\
0.85 \\
\text { ND } \\
\text { ND } \\
\text { ND } \\
\text { ND } \\
\text { ND } \\
\text { ND } \\
\text { ND } \\
\text { ND } \\
\text { ND } \\
\text { ND } \\
\text { ND } \\
\text { ND } \\
\text { ND } \\
\text { ND } \\
\text { ND } \\
\text { ND } \\
\text { ND } \\
\text { ND } \\
\text { ND } \\
\text { ND } \\
\text { ND } \\
\text { ND } \\
\text { ND } \\
\text { ND } \\
\text { ND } \\
119 \\
45.3 \\
75.0 \\
74.4 \\
\text { ND } \\
\text { ND } \\
\text { ND } \\
\text { ND } \\
\text { ND } \\
\text { ND } \\
\text { ND } \\
\text { ND } \\
\text { ND } \\
\text { ND } \\
\text { ND } \\
\text { ND } \\
\text { ND } \\
\text { ND } \\
\text { ND } \\
\text { ND } \\
\text { ND } \\
\text { ND }\end{array}$ & $\begin{array}{l}0.010 \\
0.010 \\
0.010 \\
10 \\
10 \\
10 \\
10 \\
10 \\
10 \\
10 \\
10 \\
10 \\
10 \\
10 \\
10 \\
5.0 \\
0.5 \\
0.5 \\
0.5 \\
0.5 \\
5.0 \\
0.5 \\
0.5 \\
0.5 \\
0.5 \\
10 \\
10 \\
10 \\
10 \\
24.7 \\
27.0 \\
27.0 \\
27.0 \\
27.0 \\
5.0 \\
0.5 \\
0.5 \\
0.5 \\
0.5 \\
42.5 \\
11.0 \\
11.0 \\
11.0 \\
11.0 \\
1.0 \\
1.0 \\
1.0 \\
1.0 \\
1.0 \\
1.0 \\
1.0 \\
1.0 \\
1.0 \\
1.0 \\
1.0 \\
1.0 \\
1.0 \\
1.0 \\
1.0 \\
1.0 \\
1.0 \\
\end{array}$ & 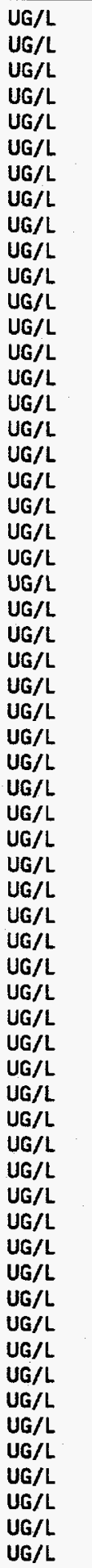 & $\begin{array}{l}\text { USATHAMA } \\
\text { USATHAMA } \\
\text { USATHAMA } \\
\text { EPA CLP } \\
\text { EPA CLP } \\
\text { EPA CLP } \\
\text { EPA CLP } \\
\text { EPA CLP } \\
\text { EPA CLP } \\
\text { EPA CLP } \\
\text { EPA CLP } \\
\text { EPA CLP } \\
\text { EPA CLP } \\
\text { EPA CLP } \\
\text { EPA CLP } \\
\text { EPA } 8310 \\
\text { EPA } 8310 \\
\text { EPA } 8310 \\
\text { SW846 } 8310 \\
\text { SW846 } 8310 \\
\text { EPA } 8310 \\
\text { EPA } 8310 \\
\text { EPA } 8310 \\
\text { SH846 } 8310 \\
\text { SW846 } 8310 \\
\text { EPA CLP } \\
\text { EPA CLP } \\
\text { EPA CLP } \\
\text { EPA CLP } \\
\text { EPA CLP } \\
\text { EPA } 6010 A \\
\text { EPA } 6010 A \\
\text { EPA } 6010 A \\
\text { EPA } 6010 A \\
\text { EPA } 8310 \\
\text { EPA } 8310 \\
\text { EPA } 8310 \\
\text { SH846 } 8310 \\
\text { SH846 } 8310 \\
\text { EPA CLP } \\
\text { EPA } 6010 A \\
\text { EPA } 6010 A \\
\text { EPA } 6010 A \\
\text { EPA } 6010 A \\
\text { EPA } 8080 A \\
\text { EPA } 8081 \\
\text { EPA } 8081 \\
\text { EPA } 8081 \\
\text { EPA } 8081 \\
\text { EPA } 8080 A \\
\text { EPA } 8081 \\
\text { EPA } 8081 \\
\text { EPA } 8081 \\
\text { EPA } 8081 \\
\text { EPA } 8080 A \\
\text { EPA } 8081 \\
\text { EPA } 8081 \\
\text { EPA } 8081 \\
\text { EPA } 8081 \\
\text { EPA } 8080 A \\
\text { EPA } 8081\end{array}$ \\
\hline
\end{tabular}




\begin{tabular}{|c|c|c|c|c|c|c|}
\hline WSSRAP_ID & DATE_SAM & PARAMETER & CONC & $\mathrm{DL}$ & UNITS & METHOD \\
\hline 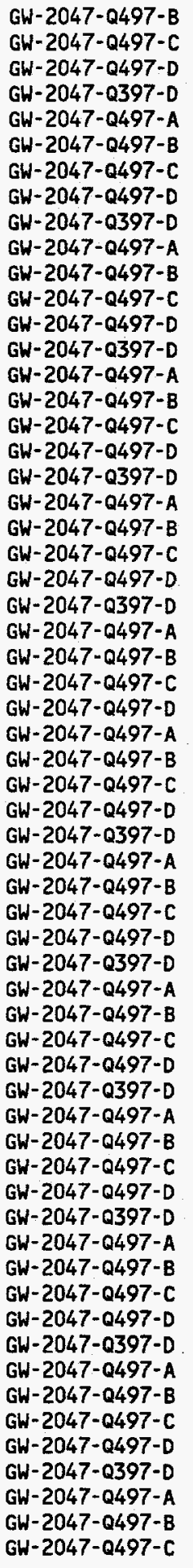 & $\begin{array}{l}10 / 07 / 97 \\
10 / 08 / 97 \\
10 / 09 / 97 \\
07 / 11 / 97 \\
10 / 02 / 97 \\
10 / 07 / 97 \\
10 / 08 / 97 \\
10 / 09 / 97 \\
07 / 11 / 97 \\
10 / 02 / 97 \\
10 / 07 / 97 \\
10 / 08 / 97 \\
10 / 09 / 97 \\
07 / 11 / 97 \\
10 / 02 / 97 \\
10 / 07 / 97 \\
10 / 08 / 97 \\
10 / 09 / 97 \\
07 / 11 / 97 \\
10 / 02 / 97 \\
10 / 07 / 97 \\
10 / 08 / 97 \\
10 / 09 / 97 \\
07 / 11 / 97 \\
10 / 02 / 97 \\
10 / 07 / 97 \\
10 / 08 / 97 \\
10 / 09 / 97 \\
10 / 02 / 97 \\
10 / 07 / 97 \\
10 / 08 / 97 \\
10 / 09 / 97 \\
07 / 11 / 97 \\
10 / 02 / 97 \\
10 / 07 / 97 \\
10 / 08 / 97 \\
10 / 09 / 97 \\
07 / 11 / 97 \\
10 / 02 / 97 \\
10 / 07 / 97 \\
10 / 08 / 97 \\
10 / 09 / 97 \\
07 / 11 / 97 \\
10 / 02 / 97 \\
10 / 07 / 97 \\
10 / 08 / 97 \\
10 / 09 / 97 \\
07 / 11 / 97 \\
10 / 02 / 97 \\
10 / 07 / 97 \\
10 / 08 / 97 \\
10 / 09 / 97 \\
07 / 11 / 97 \\
10 / 02 / 97 \\
10 / 07 / 97 \\
10 / 08 / 97 \\
10 / 09 / 97 \\
07 / 111 / 97 \\
10 / 02 / 97 \\
10 / 07 / 97 \\
10 / 08 / 97\end{array}$ & 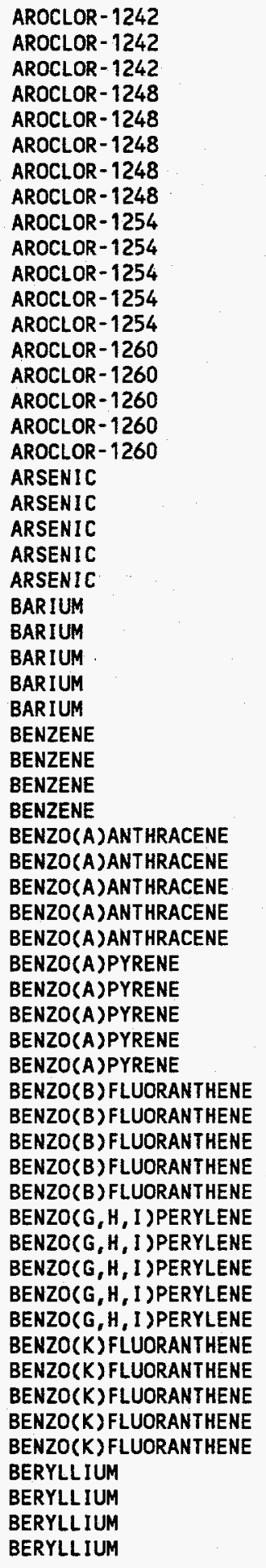 & $\begin{array}{l}\text { ND } \\
\text { ND } \\
\text { ND } \\
\text { ND } \\
\text { ND } \\
\text { ND } \\
\text { ND } \\
\text { ND } \\
\text { ND } \\
\text { ND } \\
\text { ND } \\
\text { ND } \\
\text { ND } \\
\text { ND } \\
\text { ND } \\
\text { ND } \\
\text { ND } \\
\text { ND } \\
\text { ND } \\
\text { ND } \\
\text { 216 } \\
\text { 288 } \\
\text { 306 } \\
\text { 315 } \\
\text { 322 } \\
\text { ND } \\
\text { ND } \\
\text { ND } \\
\text { ND } \\
\text { ND } \\
\text { ND } \\
\text { ND } \\
\text { ND } \\
\text { ND } \\
\text { ND } \\
\text { ND } \\
\text { ND } \\
\text { ND } \\
\text { ND } \\
\text { ND } \\
\text { ND } \\
\text { ND } \\
\text { ND } \\
\text { ND } \\
\text { ND } \\
\text { ND } \\
\text { ND } \\
\text { ND } \\
\text { ND } \\
\text { ND }\end{array}$ & $\begin{array}{l}1.0 \\
1.0 \\
1.0 \\
1.0 \\
1.0 \\
1.0 \\
1.0 \\
1.0 \\
1.0 \\
1.0 \\
1.0 \\
1.0 \\
1.0 \\
1.0 \\
1.0 \\
1.0 \\
1.0 \\
1.0 \\
1.7 \\
4.0 \\
4.0 \\
4.0 \\
4.0 \\
0.60 \\
2.0 \\
2.0 \\
2.0 \\
2.0 \\
10 \\
10 \\
10 \\
110 \\
5.0 \\
0.5 \\
0.5 \\
0.5 \\
0.5 \\
1.0 \\
0.0 \\
0.0 \\
0.0 \\
0.0 \\
0.0 \\
0.5 \\
0.5 \\
0.5 \\
0.5 \\
0.5 \\
0.0 \\
0.5 \\
0\end{array}$ & 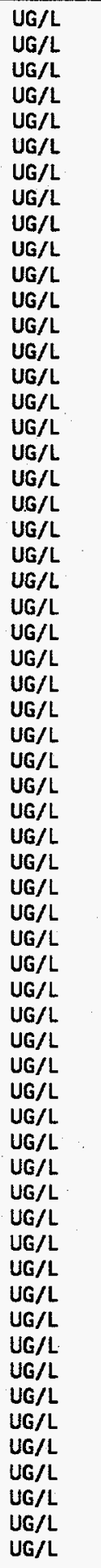 & $\begin{array}{l}\text { EPA } 8081 \\
\text { EPA } 8081 \\
\text { EPA } 8081 \\
\text { EPA } 8080 A \\
\text { EPA } 8081 \\
\text { EPA } 8081 \\
\text { EPA } 8081 \\
\text { EPA } 8081 \\
\text { EPA } 8080 A \\
\text { EPA } 8081 \\
\text { EPA } 8081 \\
\text { EPA } 8081 \\
\text { EPA } 8081 \\
\text { EPA } 8080 A \\
\text { EPA } 8081 \\
\text { EPA } 8081 \\
\text { EPA } 8081 \\
\text { EPA } 8081 \\
\text { EPA } C L P \\
\text { EPA } 6010 A \\
\text { EPA } 6010 A \\
\text { EPA } 6010 A \\
\text { EPA } 6010 A \\
\text { EPA CLP } \\
\text { EPA } 6010 A \\
\text { EPA } 6010 A \\
\text { EPA } 6010 A \\
\text { EPA } 6010 A \\
\text { EPA CLP } \\
\text { EPA CLP } \\
\text { EPA CLP } \\
\text { EPA CLP } \\
\text { EPA } 8310 \\
\text { EPA } 8310 \\
\text { EPA } 8310 \\
\text { SW846 } 8310 \\
\text { SW846 } 8310 \\
\text { EPA } 8310 \\
\text { EPA } 8310 \\
\text { EPA } 8310 \\
\text { SW846 } 8310 \\
\text { SW846 } 8310 \\
\text { EPA } 8310 \\
\text { EPA } 8310 \\
\text { EPA } 8310 \\
\text { SWB46 } 8310 \\
\text { SW846 } 8310 \\
\text { EPA } 8310 \\
\text { EPA } 8310 \\
\text { EPA } 8310 \\
\text { SW846 } 8310 \\
\text { SW846 } 8310 \\
\text { EPA } 8310 \\
\text { EPA } 8310 \\
\text { EPA } 8310 \\
\text { SW846 } 8310 \\
\text { SW846 } 8310 \\
\text { EPA CLP } \\
\text { EPA } 6010 A \\
\text { EPA } 6010 A \\
\text { EPA } 6010 A \\
\end{array}$ \\
\hline
\end{tabular}




\begin{tabular}{|c|c|c|c|c|c|c|}
\hline WSSRAP_ID & DATE_SAM & PARAMETER & CONC & $D L$ & UNITS & METHOD \\
\hline 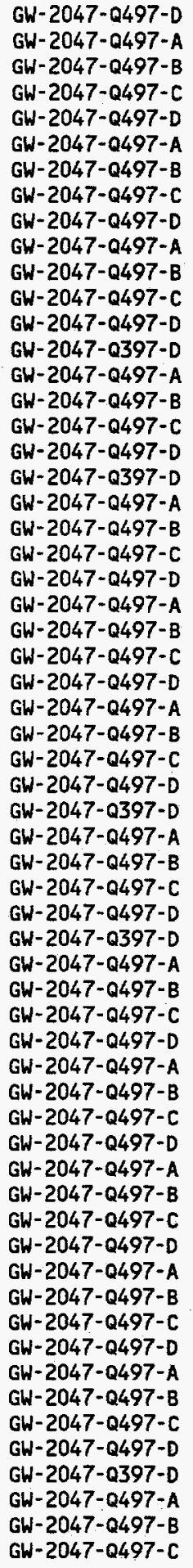 & $\begin{array}{l}10 / 09 / 97 \\
10 / 02 / 97 \\
10 / 07 / 97 \\
10 / 08 / 97 \\
10 / 09 / 97 \\
10 / 02 / 97 \\
10 / 07 / 97 \\
10 / 08 / 97 \\
10 / 09 / 97 \\
10 / 02 / 97 \\
10 / 07 / 97 \\
10 / 08 / 97 \\
10 / 09 / 97 \\
07 / 11 / 97 \\
10 / 02 / 97 \\
10 / 07 / 97 \\
10 / 08 / 97 \\
10 / 09 / 97 \\
07 / 11 / 97 \\
10 / 02 / 97 \\
10 / 07 / 97 \\
10 / 08 / 97 \\
10 / 09 / 97 \\
10 / 02 / 97 \\
10 / 07 / 97 \\
10 / 08 / 97 \\
10 / 09 / 97 \\
10 / 02 / 97 \\
10 / 07 / 97 \\
10 / 08 / 97 \\
10 / 09 / 97 \\
07 / 11 / 97 \\
10 / 02 / 97 \\
10 / 07 / 97 \\
10 / 08 / 97 \\
10 / 09 / 97 \\
07 / 11 / 97 \\
10 / 02 / 97 \\
10 / 07 / 97 \\
10 / 08 / 97 \\
10 / 09 / 97 \\
10 / 02 / 97 \\
10 / 07 / 97 \\
10 / 08 / 97 \\
10 / 09 / 97 \\
10 / 02 / 97 \\
10 / 07 / 97 \\
10 / 08 / 97 \\
10 / 09 / 97 \\
10 / 02 / 97 \\
10 / 07 / 97 \\
10 / 08 / 97 \\
10 / 09 / 97 \\
10 / 02 / 97 \\
10 / 07 / 97 \\
10 / 08 / 97 \\
10 / 09 / 97 \\
07 / 11 / 97 \\
10 / 02 / 97 \\
10 / 07 / 97 \\
10 / 08 / 97\end{array}$ & 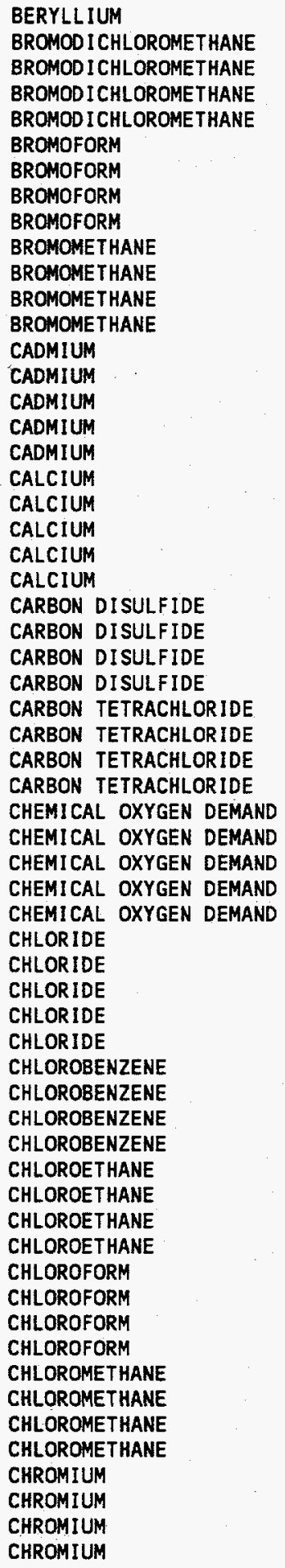 & $\begin{array}{l}\text { ND } \\
\text { ND } \\
\text { ND } \\
\text { ND } \\
\text { ND } \\
\text { ND } \\
\text { ND } \\
\text { ND } \\
\text { ND } \\
\text { ND } \\
\text { ND } \\
\text { ND } \\
\text { ND } \\
\text { ND } \\
1080 \\
\text { 11500 } \\
\text { 12100 } \\
14600 \\
13900 \\
\text { ND } \\
\text { ND } \\
\text { ND } \\
\text { ND } \\
\text { ND } \\
\text { ND } \\
\text { ND } \\
\text { ND } \\
\text { ND } \\
\text { ND } \\
\text { ND } \\
\text { ND } \\
\text { ND } \\
8.49 \\
\text { ND } \\
\text { ND } \\
\text { ND } \\
7.18 \\
\text { ND } \\
\text { ND } \\
\text { ND } \\
\text { ND } \\
\text { ND } \\
\text { ND } \\
\text { ND } \\
\text { ND } \\
\text { ND } \\
\text { ND } \\
\text { NO }\end{array}$ & $\begin{array}{l}1.0 \\
10 \\
10 \\
10 \\
10 \\
10 \\
10 \\
10 \\
10 \\
10 \\
10 \\
10 \\
10 \\
10 \\
10 \\
2.9 \\
2.0 \\
2.0 \\
2.0 \\
2.0 \\
53 \\
13.0 \\
13\end{array}$ & 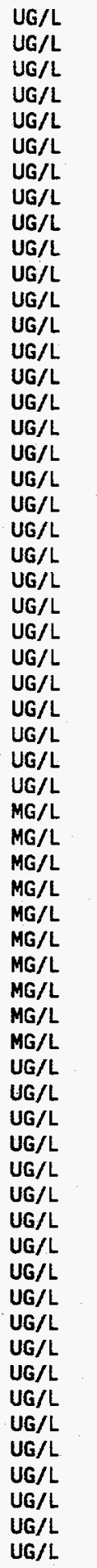 & 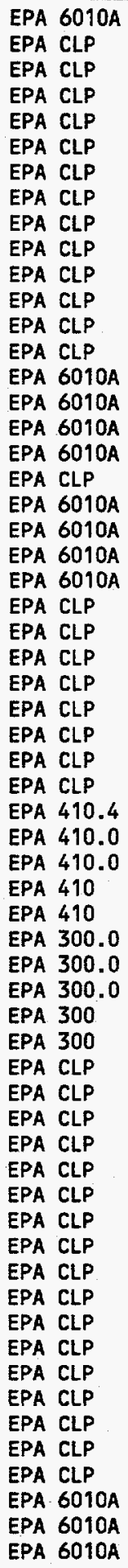 \\
\hline
\end{tabular}




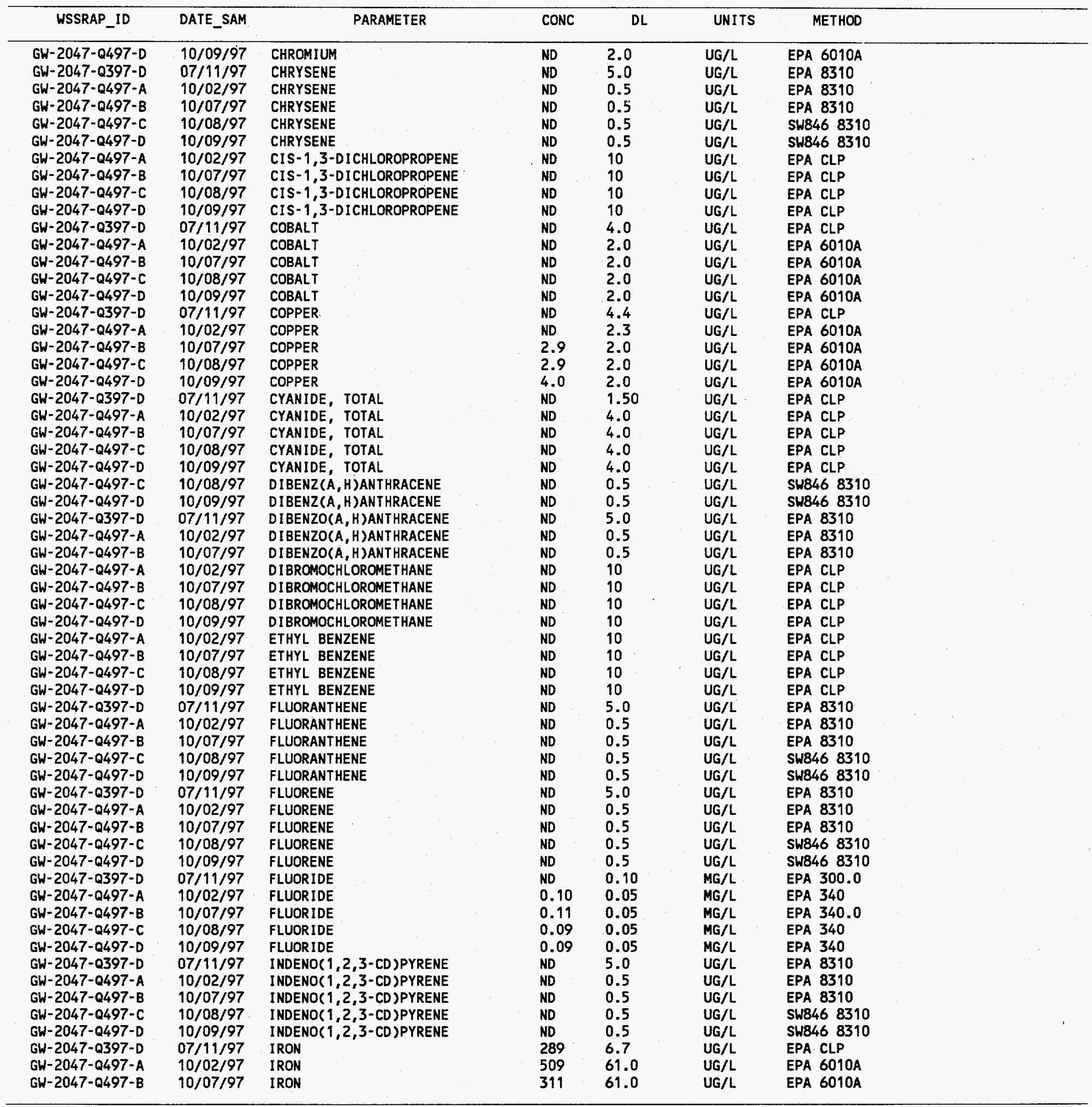




\begin{tabular}{|c|c|c|c|c|c|c|}
\hline WSSRAP_ID & DATE_SAM & PARAMETER & CONC & DL & UNITS & METHOD \\
\hline 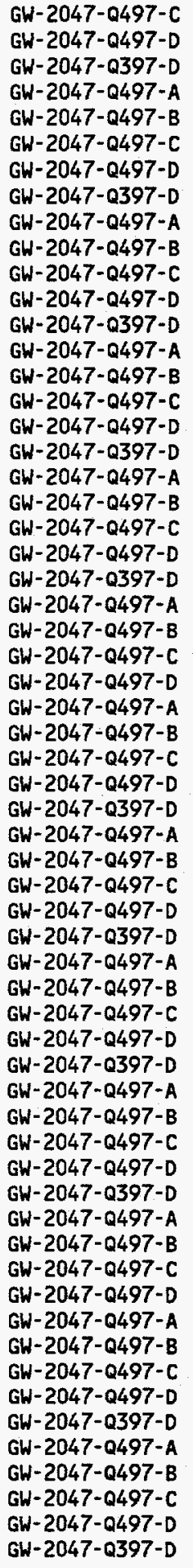 & $\begin{array}{l}10 / 08 / 97 \\
10 / 09 / 97 \\
07 / 11 / 97 \\
10 / 02 / 97 \\
10 / 07 / 97 \\
10 / 08 / 97 \\
10 / 09 / 97 \\
07 / 11 / 97 \\
10 / 02 / 97 \\
10 / 07 / 97 \\
10 / 08 / 97 \\
10 / 09 / 97 \\
07 / 11 / 97 \\
10 / 02 / 97 \\
10 / 07 / 97 \\
10 / 08 / 97 \\
10 / 09 / 97 \\
07 / 11 / 97 \\
10 / 02 / 97 \\
10 / 07 / 97 \\
10 / 08 / 97 \\
10 / 09 / 97 \\
07 / 11 / 97 \\
10 / 02 / 97 \\
10 / 07 / 97 \\
10 / 08 / 97 \\
10 / 09 / 97 \\
10 / 02 / 97 \\
10 / 07 / 97 \\
10 / 08 / 97 \\
10 / 09 / 97 \\
07 / 11 / 97 \\
10 / 02 / 97 \\
10 / 07 / 97 \\
10 / 08 / 97 \\
10 / 09 / 97 \\
07 / 11 / 97 \\
10 / 02 / 97 \\
10 / 07 / 97 \\
10 / 08 / 97 \\
10 / 09 / 97 \\
07 / 11 / 97 \\
10 / 02 / 97 \\
10 / 07 / 97 \\
10 / 08 / 97 \\
10 / 09 / 97 \\
07 / 11 / 97 \\
10 / 02 / 97 \\
10 / 07 / 97 \\
10 / 08 / 97 \\
10 / 09 / 97 \\
10 / 02 / 97 \\
10 / 07 / 97 \\
10 / 08 / 97 \\
10 / 09 / 97 \\
07 / 11 / 97 \\
10 / 02 / 97 \\
10 / 07 / 97 \\
10 / 08 / 97 \\
10 / 09 / 97 \\
07 / 11 / 97\end{array}$ & 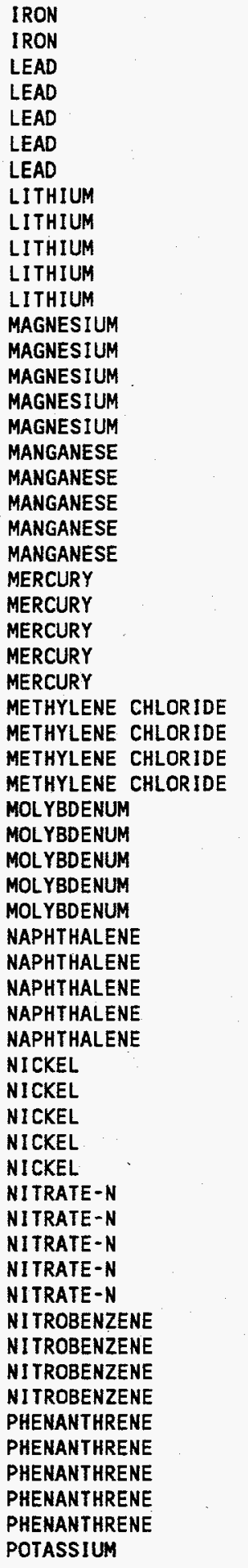 & 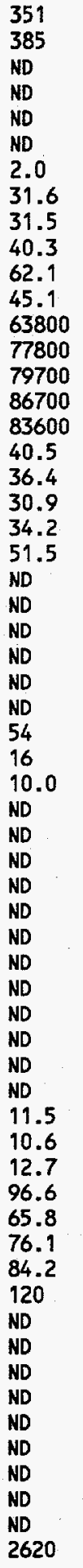 & $\begin{array}{l}61.0 \\
61.0 \\
1.8 \\
2.0 \\
2.0 \\
2.0 \\
2.0 \\
5.2 \\
2.0 \\
2.0 \\
2.0 \\
2.0 \\
68.0 \\
28.0 \\
28.0 \\
28.0 \\
28.0 \\
0.90 \\
2.0 \\
2.0 \\
2.0 \\
2.0 \\
0.10 \\
0.10 \\
0.10 \\
0.10 \\
0.10 \\
10 \\
10 \\
10 \\
10 \\
7.3 \\
7.0 \\
7.0 \\
7.0 \\
7.0 \\
5.0 \\
0.5 \\
0.5 \\
0.5 \\
0.5 \\
14.8 \\
10.2 \\
2.0 \\
2.0 \\
2.0 \\
25.0 \\
1.00 \\
2.00 \\
2.000 \\
4.000 \\
0.03 \\
0.030 \\
0.030 \\
0.030 \\
5.0 \\
0.5 \\
0.5 \\
0.5 \\
0.5 \\
1770 \\
\end{array}$ & 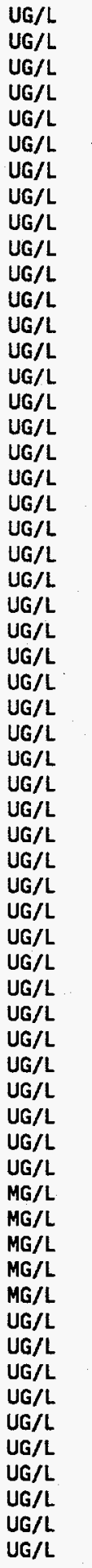 & $\begin{array}{l}\text { EPA } 6010 A \\
\text { EPA } 6010 A \\
\text { EPA CLP } \\
\text { EPA } 6010 A \\
\text { EPA } 6010 A \\
\text { EPA } 6010 A \\
\text { EPA } 6010 A \\
\text { EPA CLP } \\
\text { EPA } 6010 A \\
\text { EPA } 6010 A \\
\text { EPA } 6010 A \\
\text { EPA } 6010 A \\
\text { EPA CLP } \\
\text { EPA } 6010 A \\
\text { EPA } 6010 A \\
\text { EPA } 6010 A \\
\text { EPA } 6010 A \\
\text { EPA } \text { CLP } \\
\text { EPA } 6010 A \\
\text { EPA } 6010 A \\
\text { EPA } 6010 A \\
\text { EPA } 6010 A \\
\text { EPA } \text { CLP } \\
\text { EPA } 7470 A \\
\text { EPA } 7470 A \\
\text { EPA } 7470 \\
\text { EPA } 7470 \\
\text { EPA } \text { CLP } \\
\text { EPA CLP } \\
\text { EPA CLP } \\
\text { EPA } \text { CLP } \\
\text { EPA CLP } \\
\text { EPA } 6010 A \\
\text { EPA } 6010 A \\
\text { EPA } 6010 A \\
\text { EPA } 6010 A \\
\text { EPA } 8310 \\
\text { EPA } 8310 \\
\text { EPA } 8310 \\
\text { SW846 } 8310 \\
\text { SW846 } 8310 \\
\text { EPA CLP } \\
\text { EPA } 6010 A \\
\text { EPA } 6010 A \\
\text { EPA } 6010 A \\
\text { EPA } 6010 A \\
\text { EPA } 353.1 \\
\text { EPA } 353.1 \\
\text { EPA } 353.1 \\
\text { EPA } 353.1 \\
\text { EPA } 353.1 \\
\text { USATHAMA } \\
\text { USATHAMA } \\
\text { USATHAMA } \\
\text { USATHAMA } \\
\text { EPA } 8310 \\
\text { EPA } 8310 \\
\text { EPA } 8310 \\
\text { SW846 } 8310 \\
\text { SW846 } 8310 \\
\text { EPA CLP } \\
\end{array}$ \\
\hline
\end{tabular}




\begin{tabular}{|c|c|c|c|c|c|c|}
\hline WSSRAP_ID & DATE_SAM & PARAMETER & CONC & $\mathrm{DL}$ & UNI TS & METHOD \\
\hline 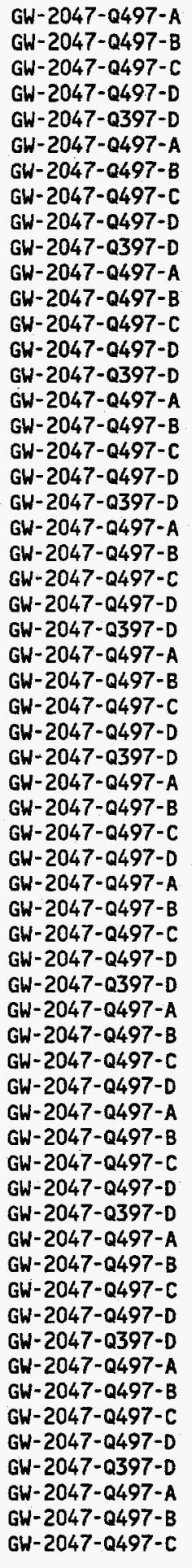 & $\begin{array}{l}10 / 02 / 97 \\
10 / 07 / 97 \\
10 / 08 / 97 \\
10 / 09 / 97 \\
07 / 11 / 97 \\
10 / 02 / 97 \\
10 / 07 / 97 \\
10 / 08 / 97 \\
10 / 09 / 97 \\
07 / 11 / 97 \\
10 / 02 / 97 \\
10 / 07 / 97 \\
10 / 08 / 97 \\
10 / 09 / 97 \\
07 / 11 / 97 \\
10 / 02 / 97 \\
10 / 07 / 97 \\
10 / 08 / 97 \\
10 / 09 / 97 \\
07 / 11 / 97 \\
10 / 02 / 97 \\
10 / 07 / 97 \\
10 / 08 / 97 \\
10 / 09 / 97 \\
07 / 11 / 97 \\
10 / 02 / 97 \\
10 / 07 / 97 \\
10 / 08 / 97 \\
10 / 09 / 97 \\
07 / 11 / 97 \\
10 / 02 / 97 \\
10 / 07 / 97 \\
10 / 08 / 97 \\
10 / 09 / 97 \\
10 / 02 / 97 \\
10 / 07 / 97 \\
10 / 08 / 97 \\
10 / 09 / 97 \\
07 / 11 / 97 \\
10 / 02 / 97 \\
10 / 07 / 97 \\
10 / 08 / 97 \\
10 / 09 / 97 \\
10 / 02 / 97 \\
10 / 07 / 97 \\
10 / 08 / 97 \\
10 / 09 / 97 \\
07 / 11 / 97 \\
10 / 02 / 97 \\
10 / 07 / 97 \\
10 / 08 / 97 \\
10 / 09 / 97 \\
07 / 11 / 97 \\
10 / 02 / 97 \\
10 / 07 / 97 \\
10 / 08 / 97 \\
10 / 09 / 97 \\
07 / 11 / 97 \\
10 / 02 / 97 \\
10 / 07 / 97 \\
10 / 08 / 97\end{array}$ & $\begin{array}{l}\text { POTASSIUM } \\
\text { POTASSIUM } \\
\text { POTASSIUM } \\
\text { POTASSIUM } \\
\text { PYRENE } \\
\text { PYRENE } \\
\text { PYRENE } \\
\text { PYRENE } \\
\text { PYRENE } \\
\text { RADIUM-226 } \\
\text { RADIUM-226 } \\
\text { RADIUM-226 } \\
\text { RADIUM-226 } \\
\text { RADIUM-226 } \\
\text { RADIUM-228 } \\
\text { RADIUM-228 } \\
\text { RADIUM-228 } \\
\text { RADIUM-228 } \\
\text { RADIUM-228 } \\
\text { SELENIUM } \\
\text { SELENIUM } \\
\text { SELENIUM } \\
\text { SELENIUM } \\
\text { SELENIUM } \\
\text { SILVER } \\
\text { SILVER } \\
\text { SILVER } \\
\text { SILVER } \\
\text { SILVER } \\
\text { SODIUM } \\
\text { SODIUM } \\
\text { SODIUM } \\
\text { SODIUM } \\
\text { SODIUM } \\
\text { STYRENE } \\
\text { STYRENE } \\
\text { STYRENE } \\
\text { STYRENE } \\
\text { SULFATE } \\
\text { SULFATE } \\
\text { SULFATE } \\
\text { SULFATE } \\
\text { SULFATE } \\
\text { TETRACHLOROETHENE } \\
\text { TETRACHLOROETHENE } \\
\text { TETRACHLOROETHENE } \\
\text { TETRACHLOROETHENE } \\
\text { THALLIUM } \\
\text { THALLIUM } \\
\text { THALLIUM } \\
\text { THALLIUM } \\
\text { THALLIUM } \\
\text { THORIUM-228 } \\
\text { THORIUM-228 } \\
\text { THORIUM-228 } \\
\text { THORIUM-228 } \\
\text { THORIUM-228 } \\
\text { THORIUM-230 } \\
\text { THORIUM-230 } \\
\text { THORIUM-230 } \\
\text { THORIUM-230 } \\
\text { THENG }\end{array}$ & $\begin{array}{l}2400 \\
2700 \\
3380 \\
2840 \\
\text { ND } \\
\text { ND } \\
\text { ND } \\
\text { ND } \\
\text { ND } \\
0.311 \\
0.290 \\
0.622 \\
10.21 \\
10.23 \\
0.647 \\
C 0.61 \\
C 0.70 \\
C 0.12 \\
\text { C. } \\
4.0 \\
\text { ND } \\
\text { ND } \\
\text { ND } \\
\text { ND } \\
\text { ND } \\
\text { ND } \\
\text { ND } \\
\text { ND } \\
\text { ND } \\
26600 \\
28400 \\
30700 \\
36500 \\
32500 \\
\text { ND } \\
\text { ND } \\
\text { ND } \\
\text { ND } \\
33.8 \\
26.1 \\
24.7 \\
28.8 \\
29.4 \\
\text { ND } \\
\text { ND } \\
\text { ND } \\
\text { ND } \\
\text { ND } \\
\text { ND } \\
\text { ND } \\
\text { ND } \\
\text { ND } \\
\text { ND } \\
\text { C0.22 } \\
\text { C0.09 } \\
\text { C0.02 } \\
\text { ND } \\
\text { C0.12 } \\
0.480 \\
0.426 \\
\text { C0.03 }\end{array}$ & 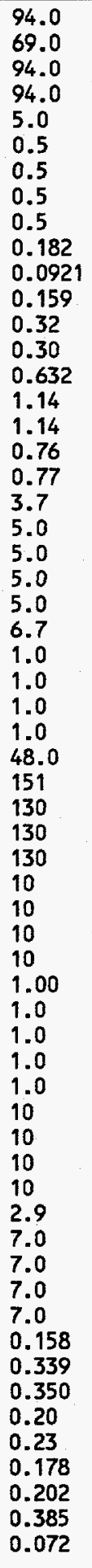 & 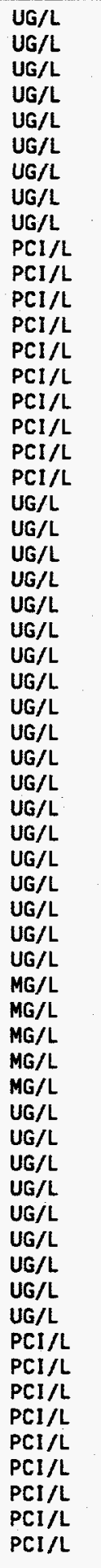 & 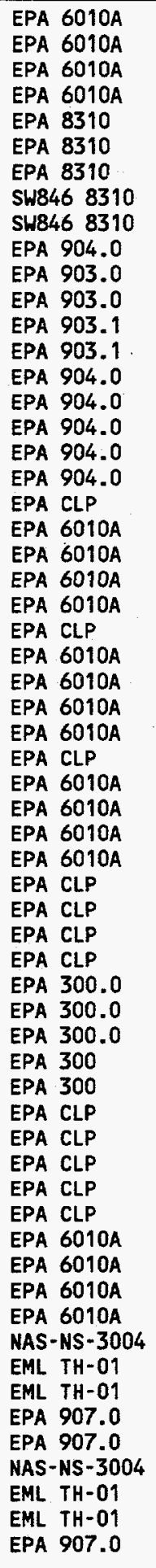 \\
\hline
\end{tabular}




\begin{tabular}{|c|c|c|c|c|c|c|}
\hline WSSRAP_ID & DATE_SAM & PARAMETER & CONC & $D L$ & UNITS & METHOD \\
\hline 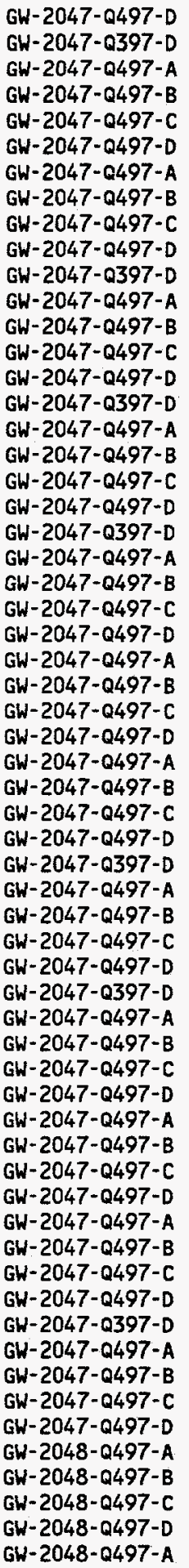 & $\begin{array}{l}10 / 09 / 97 \\
07 / 11 / 97 \\
10 / 02 / 97 \\
10 / 07 / 97 \\
10 / 08 / 97 \\
10 / 09 / 97 \\
10 / 02 / 97 \\
10 / 07 / 97 \\
10 / 08 / 97 \\
10 / 09 / 97 \\
07 / 11 / 97 \\
10 / 02 / 97 \\
10 / 07 / 97 \\
10 / 08 / 97 \\
10 / 09 / 97 \\
07 / 11 / 97 \\
10 / 02 / 97 \\
10 / 07 / 97 \\
10 / 08 / 97 \\
10 / 09 / 97 \\
07 / 11 / 97 \\
10 / 02 / 97 \\
10 / 07 / 97 \\
10 / 08 / 97 \\
10 / 09 / 97 \\
10 / 02 / 97 \\
10 / 07 / 97 \\
10 / 08 / 97 \\
10 / 09 / 97 \\
10 / 02 / 97 \\
10 / 07 / 97 \\
10 / 08 / 97 \\
10 / 09 / 97 \\
07 / 11 / 97 \\
10 / 02 / 97 \\
10 / 07 / 97 \\
10 / 08 / 97 \\
10 / 09 / 97 \\
07 / 11 / 97 \\
10 / 02 / 97 \\
10 / 07 / 97 \\
10 / 08 / 97 \\
10 / 09 / 97 \\
10 / 02 / 97 \\
10 / 07 / 97 \\
10 / 08 / 97 \\
10 / 09 / 97 \\
10 / 02 / 97 \\
10 / 07 / 97 \\
10 / 08 / 97 \\
10 / 09 / 97 \\
07 / 11 / 97 \\
10 / 02 / 97 \\
10 / 07 / 97 \\
10 / 08 / 97 \\
10 / 09 / 97 \\
10 / 01 / 97 \\
10 / 01 / 97 \\
10 / 01 / 97 \\
10 / 01 / 97 \\
10 / 01 / 97\end{array}$ & 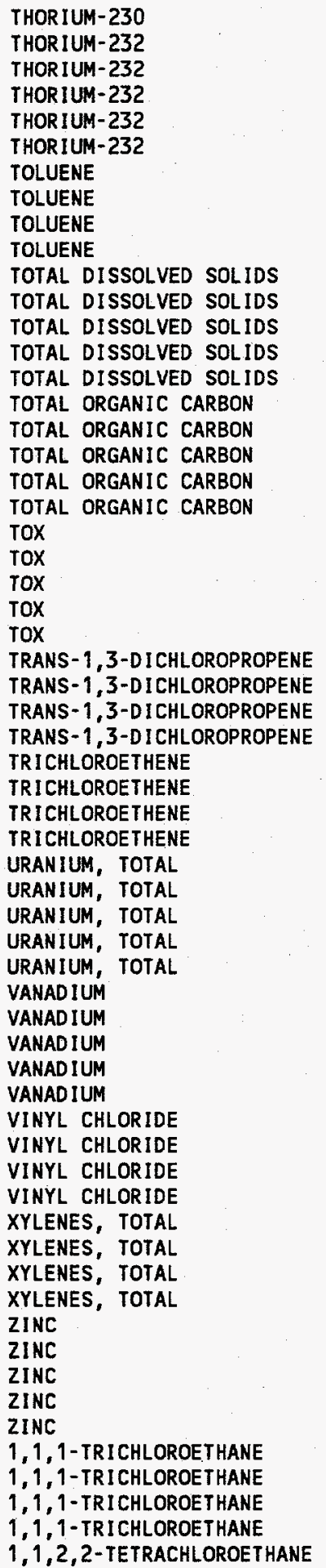 & 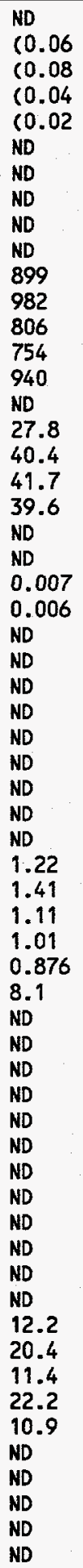 & 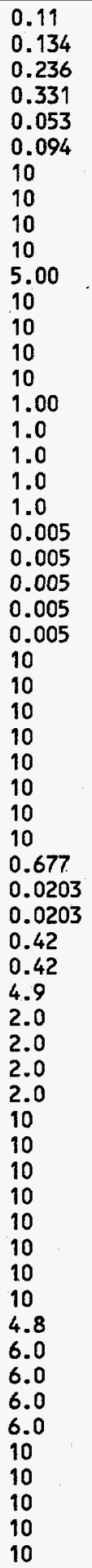 & 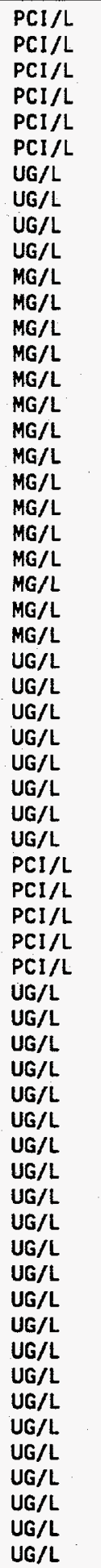 & $\begin{array}{l}\text { EPA } 907.0 \\
\text { NAS-NS-3004 } \\
\text { EML TH-01 } \\
\text { EML } T H-01 \\
\text { EPA } 907.0 \\
\text { EPA } 907.0 \\
\text { EPA CLP } \\
\text { EPA CLP } \\
\text { EPA CLP } \\
\text { EPA CLP } \\
\text { EPA } 160.1 \\
\text { EPA } 160.1 \\
\text { EPA } 160.1 \\
\text { EPA } 160.1 \\
\text { EPA } 160.1 \\
\text { EPA } 415.1 \\
\text { EPA } 415.1 \\
\text { EPA } 415.1 \\
\text { EPA } 415.1 \\
\text { EPA } 415.1 \\
\text { EPA } 9020 \\
\text { EPA } 9020 A \\
\text { EPA } 9020 A \\
\text { EPA } 9020 A \\
\text { EPA } 9020 A \\
\text { EPA CLP } \\
\text { EPA CLP } \\
\text { EPA CLP } \\
\text { EPA CLP } \\
\text { EPA CLP } \\
\text { EPA CLP } \\
\text { EPA CLP } \\
\text { EPA CLP } \\
\text { ASTM } 5174-91 \\
\text { ASTM } 5174-91 \\
\text { ASTM } 5174-91 \\
\text { ASTM } 5174-91 \\
\text { ASTM } 5174-91 \\
\text { EPA CLP } \\
\text { EPA } 6010 A \\
\text { EPA } 6010 A \\
\text { EPA } 6010 A \\
\text { EPA } 6010 A \\
\text { EPA CLP } \\
\text { EPA CLP } \\
\text { EPA CLP } \\
\text { EPA CLP } \\
\text { EPA CLP } \\
\text { EPA CLP } \\
\text { EPA CLP } \\
\text { EPA CLP } \\
\text { EPA CLP } \\
\text { EPA } 6010 A \\
\text { EPA } 6010 A \\
\text { EPA } 6010 A \\
\text { EPA } 6010 A \\
\text { EPA CLP } \\
\text { EPA CLP } \\
\text { EPA CLP } \\
\text { EPA CLP } \\
\text { EPA CLP }\end{array}$ \\
\hline
\end{tabular}




\begin{tabular}{|c|c|c|c|c|c|c|}
\hline WSSRAP_ID & DATE_SAM & PARAMETER & CONC & $\mathrm{DL}$ & UNITS & METHOD \\
\hline 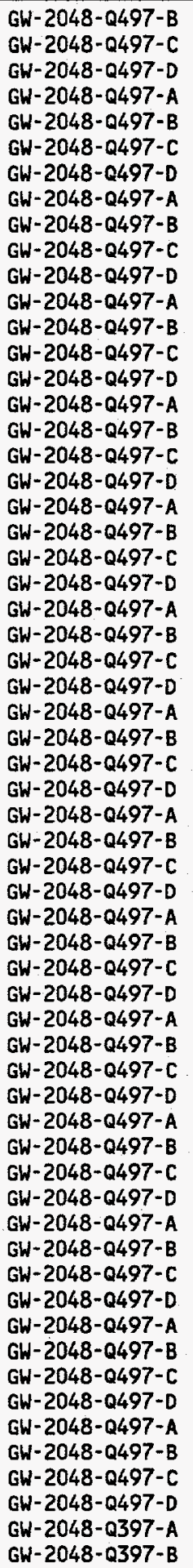 & $\begin{array}{l}10 / 01 / 97 \\
10 / 01 / 97 \\
10 / 01 / 97 \\
10 / 01 / 97 \\
10 / 01 / 97 \\
10 / 01 / 97 \\
10 / 01 / 97 \\
10 / 01 / 97 \\
10 / 01 / 97 \\
10 / 01 / 97 \\
10 / 01 / 97 \\
10 / 01 / 97 \\
10 / 01 / 97 \\
10 / 01 / 97 \\
10 / 01 / 97 \\
10 / 01 / 97 \\
10 / 01 / 97 \\
10 / 01 / 97 \\
10 / 01 / 97 \\
10 / 01 / 97 \\
10 / 01 / 97 \\
10 / 01 / 97 \\
10 / 01 / 97 \\
10 / 01 / 97 \\
10 / 01 / 97 \\
10 / 01 / 97 \\
10 / 01 / 97 \\
10 / 01 / 97 \\
10 / 01 / 97 \\
10 / 01 / 97 \\
10 / 01 / 97 \\
10 / 01 / 97 \\
10 / 01 / 97 \\
10 / 01 / 97 \\
10 / 01 / 97 \\
10 / 01 / 97 \\
10 / 01 / 97 \\
10 / 01 / 97 \\
10 / 01 / 97 \\
10 / 01 / 97 \\
10 / 01 / 97 \\
10 / 01 / 97 \\
10 / 01 / 97 \\
10 / 01 / 97 \\
10 / 01 / 97 \\
10 / 01 / 97 \\
10 / 01 / 97 \\
10 / 01 / 97 \\
10 / 01 / 97 \\
10 / 01 / 97 \\
10 / 01 / 97 \\
10 / 01 / 97 \\
10 / 01 / 97 \\
10 / 01 / 97 \\
10 / 01 / 97 \\
10 / 01 / 97 \\
10 / 01 / 97 \\
10 / 01 / 97 \\
10 / 01 / 97 \\
07 / 01 / 97 \\
07 / 01 / 97\end{array}$ & 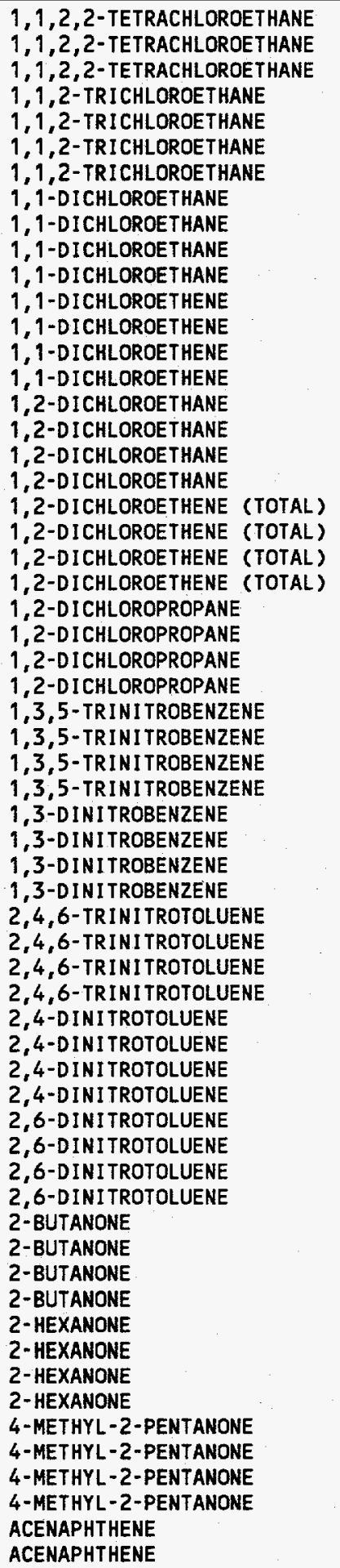 & $\begin{array}{l}\text { ND } \\
\text { ND } \\
\text { ND } \\
\text { ND } \\
\text { ND } \\
\text { ND } \\
\text { ND } \\
\text { ND } \\
\text { ND } \\
\text { ND } \\
\text { ND } \\
\text { ND } \\
\text { ND } \\
\text { ND } \\
\text { ND } \\
\text { ND } \\
\text { ND } \\
\text { ND } \\
\text { ND } \\
\text { ND } \\
\text { ND } \\
\text { ND } \\
\text { ND } \\
\text { ND } \\
\text { ND } \\
\text { ND } \\
\text { ND } \\
\text { ND } \\
\text { ND } \\
\text { ND } \\
\text { ND } \\
\text { ND } \\
\text { ND } \\
\text { ND } \\
\text { ND } \\
\text { ND } \\
\text { ND } \\
\text { ND } \\
\text { ND } \\
\text { ND } \\
\text { ND } \\
\text { ND } \\
\text { ND } \\
\text { ND } \\
\text { ND } \\
\text { ND } \\
\text { ND } \\
\text { ND } \\
\text { ND } \\
\text { ND } \\
\text { ND } \\
\text { ND } \\
\text { ND } \\
\text { ND } \\
\text { ND } \\
\text { ND } \\
\text { ND } \\
\text { ND } \\
\text { ND } \\
\text { ND }\end{array}$ & $\begin{array}{l}10 \\
10 \\
10 \\
10 \\
10 \\
10 \\
10 \\
10 \\
10 \\
10 \\
10 \\
10 \\
10 \\
10 \\
10 \\
10 \\
10 \\
10 \\
10 \\
10 \\
10 \\
10 \\
10 \\
10 \\
10 \\
10 \\
10 \\
0.030 \\
0.030 \\
0.030 \\
0.030 \\
0.090 \\
0.090 \\
0.090 \\
0.090 \\
0.030 \\
0.030 \\
0.030 \\
0.030 \\
0.030 \\
0.030 \\
0.030 \\
0.030 \\
0.010 \\
0.010 \\
0.010 \\
0.010 \\
10 \\
10 \\
10 \\
10 \\
10 \\
10 \\
10 \\
10 \\
10 \\
10 \\
10 \\
10 \\
1.00 \\
1.00 \\
\end{array}$ & 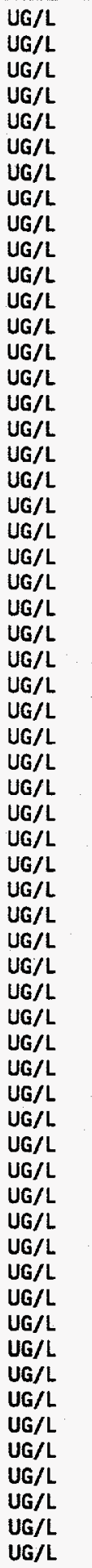 & $\begin{array}{l}\text { EPA CLP } \\
\text { EPA CLP } \\
\text { EPA CLP } \\
\text { EPA CLP } \\
\text { EPA CLP } \\
\text { EPA CLP } \\
\text { EPA CLP } \\
\text { EPA CLP } \\
\text { EPA CLP } \\
\text { EPA CLP } \\
\text { EPA CLP } \\
\text { EPA CLP } \\
\text { EPA CLP } \\
\text { EPA CLP } \\
\text { EPA CLP } \\
\text { EPA CLP } \\
\text { EPA CLP } \\
\text { EPA CLP } \\
\text { EPA CLP } \\
\text { EPA CLP } \\
\text { EPA CLP } \\
\text { EPA CLP } \\
\text { EPA CLP } \\
\text { EPA CLP } \\
\text { EPA CLP } \\
\text { EPA CLP } \\
\text { EPA CLP } \\
\text { USATHAMA } \\
\text { USATHAMA } \\
\text { USATHAMA } \\
\text { USATHAMA } \\
\text { USATHAMA } \\
\text { USATHAMA } \\
\text { USATHAMA } \\
\text { USATHAMA } \\
\text { USATHAMA } \\
\text { USATHAMA } \\
\text { USATHAMA } \\
\text { USATHAMA } \\
\text { USATHAMA } \\
\text { USATHAMA } \\
\text { USATHAMA } \\
\text { USATHAMA } \\
\text { USATHAMA } \\
\text { USATHAMA } \\
\text { USATHAMA } \\
\text { USATHAMA } \\
\text { EPA CLP } \\
\text { EPA CLP } \\
\text { EPA CLP } \\
\text { EPA CLP } \\
\text { EPA CLP } \\
\text { EPA CLP } \\
\text { EPA CLP } \\
\text { EPA CLP } \\
\text { EPA CLP } \\
\text { EPA CLP } \\
\text { EPA CLP } \\
\text { EPA CLP } \\
\text { EPA } 8270 A \\
\text { EPA } 8270 A\end{array}$ \\
\hline
\end{tabular}




\begin{tabular}{|c|c|c|c|c|c|c|}
\hline WSSRAP_ID & DATE_SAM & PARAMETER & CONC & $D L$ & UNITS & METHOD \\
\hline 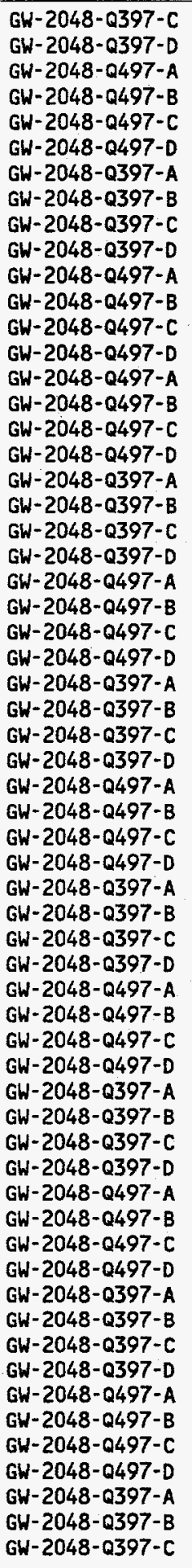 & $\begin{array}{l}07 / 01 / 97 \\
07 / 01 / 97 \\
10 / 01 / 97 \\
10 / 01 / 97 \\
10 / 01 / 97 \\
10 / 01 / 97 \\
07 / 01 / 97 \\
07 / 01 / 97 \\
07 / 01 / 97 \\
07 / 01 / 97 \\
10 / 01 / 97 \\
10 / 01 / 97 \\
10 / 01 / 97 \\
10 / 01 / 97 \\
10 / 01 / 97 \\
10 / 01 / 97 \\
10 / 01 / 97 \\
10 / 01 / 97 \\
07 / 01 / 97 \\
07 / 01 / 97 \\
07 / 01 / 97 \\
07 / 01 / 97 \\
10 / 01 / 97 \\
10 / 01 / 97 \\
10 / 01 / 97 \\
10 / 01 / 97 \\
07 / 01 / 97 \\
07 / 01 / 97 \\
07 / 01 / 97 \\
07 / 01 / 97 \\
10 / 01 / 97 \\
10 / 01 / 97 \\
10 / 01 / 97 \\
10 / 01 / 97 \\
07 / 01 / 97 \\
07 / 01 / 97 \\
07 / 01 / 97 \\
07 / 01 / 97 \\
10 / 01 / 97 \\
10 / 01 / 97 \\
10 / 01 / 97 \\
10 / 01 / 97 \\
07 / 01 / 97 \\
07 / 01 / 97 \\
07 / 01 / 97 \\
07 / 01 / 97 \\
10 / 01 / 97 \\
10 / 01 / 97 \\
10 / 01 / 97 \\
10 / 011 / 97 \\
07 / 01 / 97 \\
07 / 01 / 97 \\
07 / 01 / 97 \\
07 / 01 / 97 \\
10 / 01 / 97 \\
10 / 01 / 97 \\
10 / 01 / 97 \\
10 / 01 / 97 \\
07 / 011 / 97 \\
07 / 01 / 97 \\
07 / 01 / 97\end{array}$ & 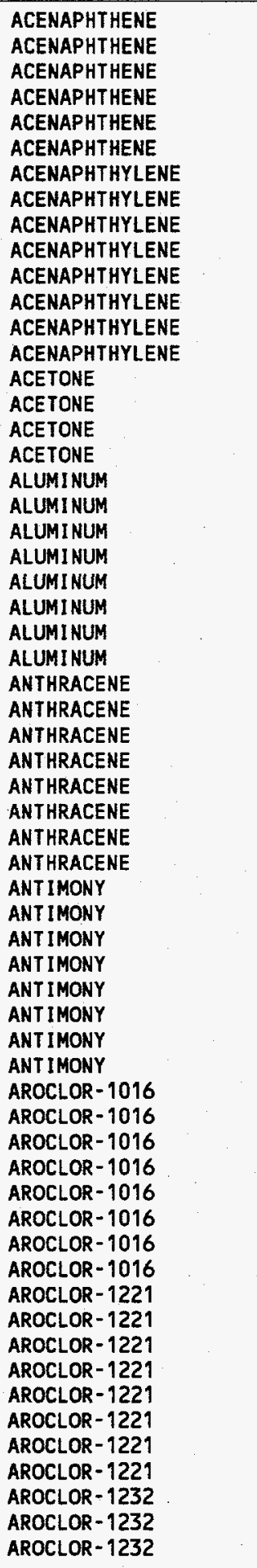 & $\begin{array}{l}\text { ND } \\
\text { ND } \\
\text { ND } \\
\text { ND } \\
\text { ND } \\
\text { ND } \\
\text { ND } \\
\text { ND } \\
\text { ND } \\
\text { ND } \\
\text { ND } \\
\text { ND } \\
\text { ND } \\
\text { ND } \\
\text { ND } \\
\text { ND } \\
\text { ND } \\
44.0 \\
49.9 \\
\text { 50.4 } \\
\text { 59.2 } \\
\text { ND } \\
36.0 \\
41.8 \\
28.4 \\
\text { ND } \\
\text { ND } \\
\text { ND } \\
\text { ND } \\
\text { ND } \\
\text { ND } \\
\text { ND } \\
\text { ND } \\
\text { ND } \\
\text { ND } \\
\text { ND } \\
\text { ND } \\
\text { ND } \\
\text { ND } \\
\text { ND } \\
\text { ND } \\
\text { ND } \\
\text { ND } \\
\text { ND } \\
\text { ND } \\
\text { ND } \\
\text { ND } \\
\text { ND } \\
\text { ND } \\
\text { ND } \\
\text { ND } \\
\text { ND } \\
\text { ND } \\
\text { ND } \\
\text { ND } \\
\text { ND } \\
\text { ND }\end{array}$ & 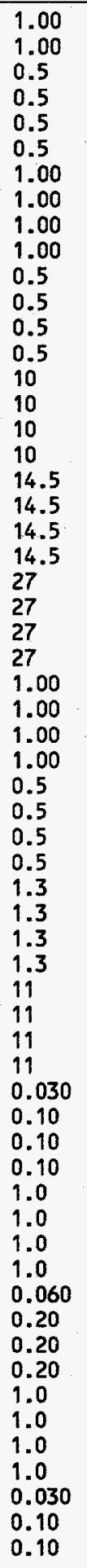 & 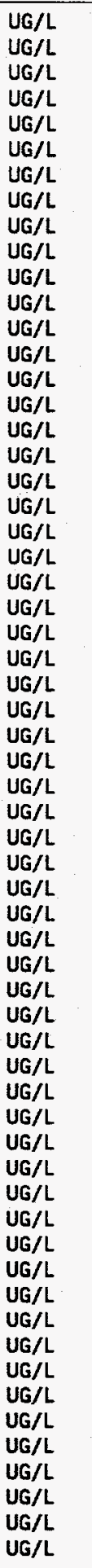 & 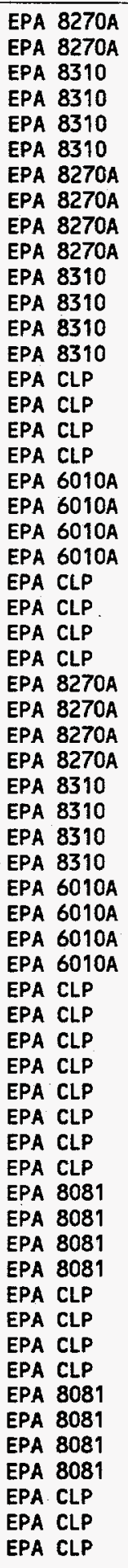 \\
\hline
\end{tabular}




\begin{tabular}{|c|c|c|c|c|c|c|}
\hline WSSRAP_ID & DATE_SAM & PARAMETER & CONC & $\mathrm{DL}$ & UNITS & METHOD \\
\hline 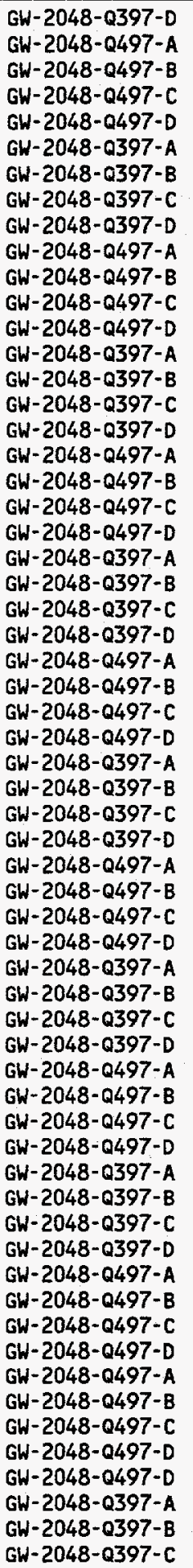 & $\begin{array}{l}07 / 01 / 97 \\
10 / 01 / 97 \\
10 / 01 / 97 \\
10 / 01 / 97 \\
10 / 01 / 97 \\
07 / 01 / 97 \\
07 / 01 / 97 \\
07 / 01 / 97 \\
07 / 01 / 97 \\
10 / 01 / 97 \\
10 / 01 / 97 \\
10 / 01 / 97 \\
10 / 01 / 97 \\
07 / 01 / 97 \\
07 / 01 / 97 \\
07 / 01 / 97 \\
07 / 01 / 97 \\
10 / 01 / 97 \\
10 / 01 / 97 \\
10 / 01 / 97 \\
10 / 01 / 97 \\
07 / 01 / 97 \\
07 / 01 / 97 \\
07 / 01 / 97 \\
07 / 01 / 97 \\
10 / 01 / 97 \\
10 / 01 / 97 \\
10 / 01 / 97 \\
10 / 01 / 97 \\
07 / 01 / 97 \\
07 / 01 / 97 \\
07 / 01 / 97 \\
07 / 01 / 97 \\
10 / 01 / 97 \\
10 / 01 / 97 \\
10 / 01 / 97 \\
10 / 01 / 97 \\
07 / 01 / 97 \\
07 / 01 / 97 \\
07 / 01 / 97 \\
07 / 01 / 97 \\
10 / 01 / 97 \\
10 / 01 / 97 \\
10 / 01 / 97 \\
10 / 01 / 97 \\
07 / 01 / 97 \\
07 / 01 / 97 \\
07 / 01 / 97 \\
07 / 01 / 97 \\
10 / 01 / 97 \\
10 / 01 / 97 \\
10 / 01 / 97 \\
10 / 01 / 97 \\
10 / 01 / 97 \\
10 / 01 / 97 \\
10 / 01 / 97 \\
10 / 01 / 97 \\
10 / 01 / 97 \\
07 / 01 / 97 \\
07 / 01 / 97 \\
07 / 01 / 97\end{array}$ & $\begin{array}{l}\text { AROCLOR-1232 } \\
\text { AROCLOR-1232 } \\
\text { AROCLOR-1232 } \\
\text { AROCLOR-1232 } \\
\text { AROCLOR-1232 } \\
\text { AROCLOR-1242 } \\
\text { AROCLOR-1242 } \\
\text { AROCLOR-1242 } \\
\text { AROCLOR-1242 } \\
\text { AROCLOR-1242 } \\
\text { AROCLOR-1242 } \\
\text { AROCLOR-1242 } \\
\text { AROCLOR-1242 } \\
\text { AROCLOR-1248 } \\
\text { AROCLOR-1248 } \\
\text { AROCLOR-1248 } \\
\text { AROCLOR-1248 } \\
\text { AROCLOR-1248 } \\
\text { AROCLOR-1248 } \\
\text { AROCLOR-1248 } \\
\text { AROCLOR-1248 } \\
\text { AROCLOR-1254 } \\
\text { AROCLOR-1254 } \\
\text { AROCLOR-1254 } \\
\text { AROCLOR-1254 } \\
\text { AROCLOR-1254 } \\
\text { AROCLOR-1254 } \\
\text { AROCLOR-1254 } \\
\text { AROCLOR-1254 } \\
\text { AROCLOR-1260 } \\
\text { AROCLOR-1260 } \\
\text { AROCLOR-1260 } \\
\text { AROCLOR-1260 } \\
\text { AROCLOR-1260 } \\
\text { AROCLOR-1260 } \\
\text { AROCLOR-1260 } \\
\text { AROCLOR-1260 } \\
\text { ARSENIC } \\
\text { ARSENIC } \\
\text { ARSENIC } \\
\text { ARSENIC } \\
\text { ARSENIC } \\
\text { ARSENIC } \\
\text { ARSENIC } \\
\text { ARSENIC } \\
\text { BARIUM } \\
\text { BARIUM } \\
\text { BARIUM } \\
\text { BARIUM } \\
\text { BARIUM } \\
\text { BARIUM } \\
\text { BARIUM } \\
\text { BARIUM } \\
\text { BENZENE } \\
\text { BENZENE } \\
\text { BENZENE } \\
\text { BENZENE } \\
\text { BENZENE, } 1,2,3-T R I C H L O R O-(T I C) ~ \\
\text { BENZO(A)ANTHRACENE } \\
\text { BENZO(A)ANTHRACENE } \\
\text { BENZO(A)ANTHRACENE } \\
\text { BA }\end{array}$ & $\begin{array}{l}\text { ND } \\
\text { ND } \\
\text { ND } \\
\text { ND } \\
\text { ND } \\
\text { ND } \\
\text { ND } \\
\text { ND } \\
\text { ND } \\
\text { ND } \\
\text { ND } \\
\text { ND } \\
\text { ND } \\
\text { ND } \\
\text { ND } \\
\text { ND } \\
\text { ND } \\
\text { ND } \\
\text { ND } \\
\text { ND } \\
\text { ND } \\
\text { ND } \\
\text { ND } \\
\text { ND } \\
\text { ND } \\
\text { ND } \\
\text { ND } \\
\text { ND } \\
\text { ND } \\
\text { ND } \\
\text { ND } \\
\text { ND } \\
\text { ND } \\
\text { ND } \\
\text { ND } \\
\text { ND } \\
\text { ND } \\
\text { ND } \\
\text { ND } \\
\text { ND } \\
\text { ND } \\
\text { ND } \\
\text { ND } \\
\text { ND } \\
\text { ND } \\
\text { ND } \\
52.0 \\
46.9 \\
41.9 \\
52.4 \\
\text { ND } \\
\text { ND } \\
\text { ND } \\
\text { ND } \\
\text { ND } \\
\text { ND } \\
\text { ND }\end{array}$ & $\begin{array}{l}0.10 \\
1.0 \\
1.0 \\
1.0 \\
1.0 \\
0.030 \\
0.10 \\
0.10 \\
0.10 \\
1.0 \\
1.0 \\
1.0 \\
1.0 \\
0.030 \\
0.10 \\
0.10 \\
0.10 \\
1.0 \\
1.0 \\
1.0 \\
1.0 \\
0.030 \\
0.10 \\
0.10 \\
0.10 \\
1.0 \\
1.0 \\
1.0 \\
1.0 \\
0.030 \\
0.10 \\
0.10 \\
0.10 \\
1.0 \\
1.0 \\
1.0 \\
1.0 \\
1.7 \\
1.7 \\
1.7 \\
1\end{array}$ & 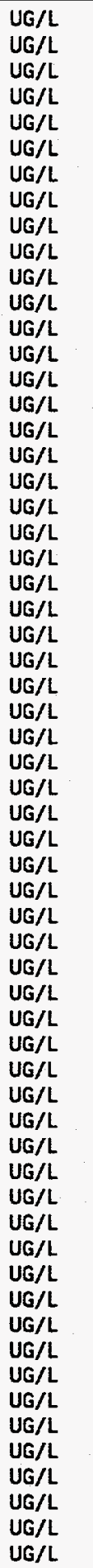 & 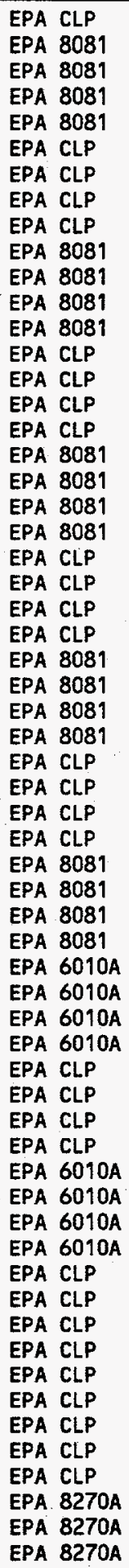 \\
\hline
\end{tabular}




\begin{tabular}{|c|c|c|c|c|c|c|}
\hline WSSRAP_ID & DATE_SAM & PARAMETER & CONC & $D L$ & UNITS & METHOD \\
\hline 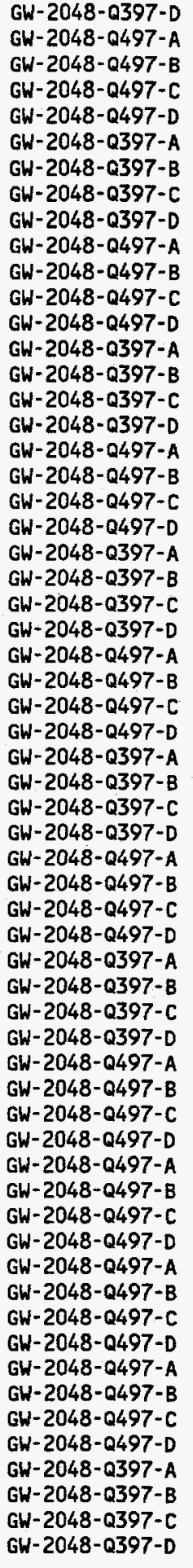 & $\begin{array}{l}07 / 01 / 97 \\
10 / 01 / 97 \\
10 / 01 / 97 \\
10 / 01 / 97 \\
10 / 01 / 97 \\
07 / 01 / 97 \\
07 / 01 / 97 \\
07 / 01 / 97 \\
07 / 01 / 97 \\
10 / 01 / 97 \\
10 / 01 / 97 \\
10 / 01 / 97 \\
10 / 01 / 97 \\
07 / 01 / 97 \\
07 / 01 / 97 \\
07 / 01 / 97 \\
07 / 01 / 97 \\
10 / 01 / 97 \\
10 / 01 / 97 \\
10 / 01 / 97 \\
10 / 01 / 97 \\
07 / 01 / 97 \\
07 / 01 / 97 \\
07 / 01 / 97 \\
07 / 01 / 97 \\
10 / 01 / 97 \\
10 / 01 / 97 \\
10 / 01 / 97 \\
10 / 01 / 97 \\
07 / 01 / 97 \\
07 / 01 / 97 \\
07 / 01 / 97 \\
07 / 01 / 97 \\
10 / 01 / 97 \\
10 / 01 / 97 \\
10 / 01 / 97 \\
10 / 01 / 97 \\
07 / 01 / 97 \\
07 / 01 / 97 \\
07 / 01 / 97 \\
07 / 01 / 97 \\
10 / 01 / 97 \\
10 / 01 / 97 \\
10 / 01 / 97 \\
10 / 01 / 97 \\
10 / 01 / 97 \\
10 / 01 / 97 \\
10 / 011 / 97 \\
10 / 01 / 97 \\
10 / 01 / 97 \\
10 / 01 / 97 \\
10 / 01 / 97 \\
10 / 01 / 97 \\
10 / 01 / 97 \\
10 / 01 / 97 \\
10 / 01 / 97 \\
10 / 01 / 97 \\
07 / 011 / 97 \\
07 / 01 / 97 \\
07 / 01 / 97 \\
07 / 01 / 97\end{array}$ & 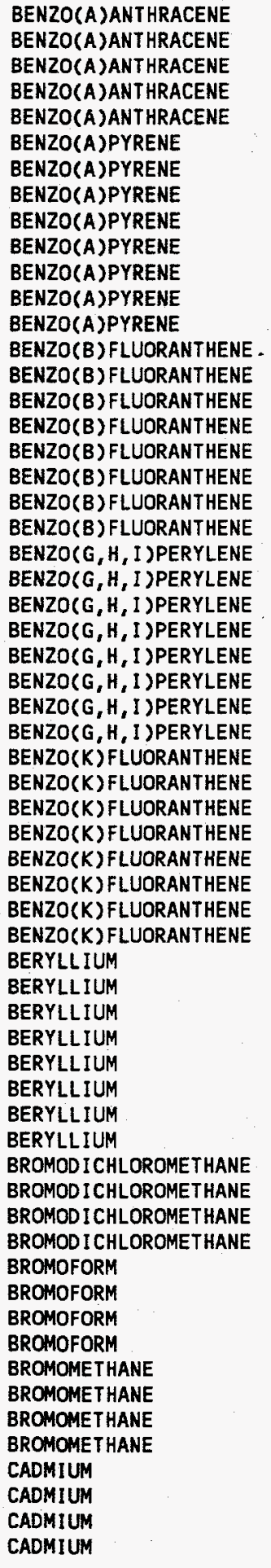 & $\begin{array}{l}\text { ND } \\
\text { ND } \\
\text { ND } \\
\text { ND } \\
\text { ND } \\
\text { ND } \\
\text { ND } \\
\text { ND } \\
\text { ND } \\
\text { ND } \\
\text { ND } \\
\text { ND } \\
\text { ND } \\
\text { ND } \\
\text { ND } \\
\text { ND } \\
\text { ND } \\
\text { ND } \\
\text { ND } \\
\text { ND } \\
\text { ND } \\
\text { ND } \\
\text { ND } \\
\text { ND } \\
\text { ND } \\
\text { ND } \\
\text { ND } \\
\text { ND } \\
\text { ND } \\
\text { ND } \\
\text { ND } \\
\text { ND } \\
\text { ND } \\
\text { ND } \\
\text { ND } \\
\text { ND } \\
\text { ND } \\
\text { ND } \\
\text { ND } \\
\text { ND } \\
\text { ND } \\
\text { ND } \\
\text { ND } \\
\text { ND } \\
\text { ND } \\
\text { ND } \\
\text { ND } \\
\text { ND } \\
\text { ND } \\
\text { ND } \\
\text { ND } \\
\text { ND } \\
\text { ND } \\
\text { ND } \\
\text { Nol }\end{array}$ & $\begin{array}{l}1.00 \\
0.5 \\
0.5 \\
0.5 \\
0.5 \\
1.00 \\
1.00 \\
1.00 \\
1.00 \\
0.5 \\
0.5 \\
0.5 \\
0.5 \\
1.00 \\
11.00 \\
1.00 \\
1.00 \\
0.5 \\
0.5 \\
0.5 \\
0.5 \\
1.00 \\
1.00 \\
1.00 \\
11.00 \\
0.5\end{array}$ & 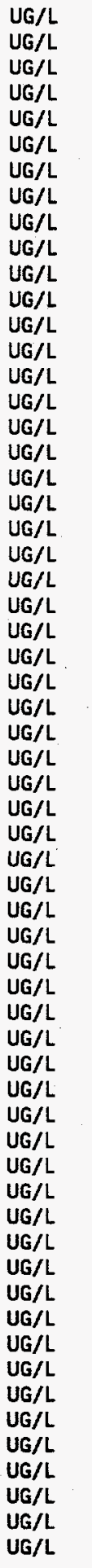 & 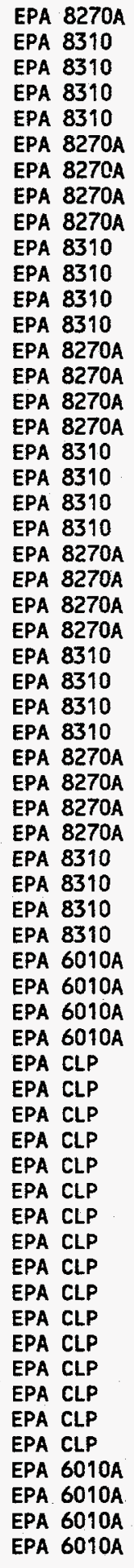 \\
\hline
\end{tabular}




\begin{tabular}{|c|c|c|c|c|c|c|}
\hline WSSRAP_ID & DATE_SAM & PARAMETER & CONC & $\mathrm{OL}$ & UNITS & METHOD \\
\hline 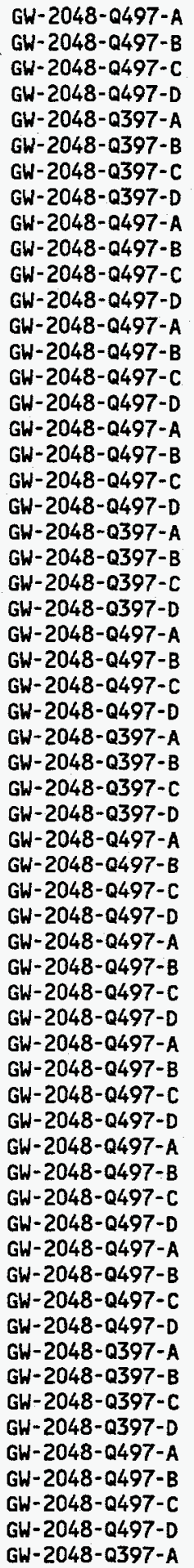 & $\begin{array}{l}10 / 01 / 97 \\
10 / 01 / 97 \\
10 / 01 / 97 \\
10 / 01 / 97 \\
07 / 01 / 97 \\
07 / 01 / 97 \\
07 / 01 / 97 \\
07 / 01 / 97 \\
10 / 01 / 97 \\
10 / 01 / 97 \\
10 / 01 / 97 \\
10 / 01 / 97 \\
10 / 01 / 97 \\
10 / 01 / 97 \\
10 / 01 / 97 \\
10 / 01 / 97 \\
10 / 01 / 97 \\
10 / 01 / 97 \\
10 / 01 / 97 \\
10 / 01 / 97 \\
07 / 01 / 97 \\
07 / 01 / 97 \\
07 / 01 / 97 \\
07 / 01 / 97 \\
10 / 01 / 97 \\
10 / 01 / 97 \\
10 / 01 / 97 \\
10 / 01 / 97 \\
07 / 01 / 97 \\
07 / 01 / 97 \\
07 / 01 / 97 \\
07 / 01 / 97 \\
10 / 01 / 97 \\
10 / 01 / 97 \\
10 / 01 / 97 \\
10 / 01 / 97 \\
10 / 01 / 97 \\
10 / 01 / 97 \\
10 / 01 / 97 \\
10 / 01 / 97 \\
10 / 01 / 97 \\
10 / 01 / 97 \\
10 / 01 / 97 \\
10 / 01 / 97 \\
10 / 01 / 97 \\
10 / 01 / 97 \\
10 / 01 / 97 \\
10 / 01 / 97 \\
10 / 01 / 97 \\
10 / 01 / 97 \\
10 / 01 / 97 \\
10 / 01 / 97 \\
07 / 01 / 97 \\
07 / 01 / 97 \\
07 / 01 / 97 \\
07 / 01 / 97 \\
10 / 01 / 97 \\
10 / 01 / 97 \\
10 / 01 / 97 \\
10 / 01 / 97 \\
07 / 01 / 97\end{array}$ & $\begin{array}{l}\text { CADMIUM } \\
\text { CADMIUM } \\
\text { CADMIUM } \\
\text { CADMIUM } \\
\text { CALCIUM } \\
\text { CALCIUM } \\
\text { CALCIUM } \\
\text { CALCIUM } \\
\text { CALCIUM } \\
\text { CALCIUM } \\
\text { CALCIUM } \\
\text { CALCIUM } \\
\text { CARBON DISULFIDE } \\
\text { CARBON DISULFIDE } \\
\text { CARBON DISULFIDE } \\
\text { CARBON DISULFIDE } \\
\text { CARBON TETRACHLORIDE } \\
\text { CARBON TETRACHLORIDE } \\
\text { CARBON TETRACHLORIDE } \\
\text { CARBON TETRACHLORIDE } \\
\text { CHEMICAL OXYGEN DEMAND } \\
\text { CHEMICAL OXYGEN DEMAND } \\
\text { CHEMICAL OXYGEN DEMAND } \\
\text { CHEMICAL OXYGEN DEMAND } \\
\text { CHEMICAL OXYGEN DEMAND } \\
\text { CHEMICAL OXYGEN DEMAND } \\
\text { CHEMICAL OXYGEN DEMAND } \\
\text { CHEMICAL OXYGEN DEMAND } \\
\text { CHLORIDE } \\
\text { CHLORIDE } \\
\text { CHLORIDE } \\
\text { CHLORIDE } \\
\text { CHLORIDE } \\
\text { CHLORIDE } \\
\text { CHLORIDE } \\
\text { CHLORIDE } \\
\text { CHLOROBENZENE } \\
\text { CHLOROBENZENE } \\
\text { CHLOROBENZENE } \\
\text { CHLOROBENZENE } \\
\text { CHLOROETHANE } \\
\text { CHLOROETHANE } \\
\text { CHLOROETHANE } \\
\text { CHLOROETHANE } \\
\text { CHLOROFORM } \\
\text { CHLOROFORM } \\
\text { CHLOROFORM } \\
\text { CHLOROFORM } \\
\text { CHLOROMETHANE } \\
\text { CHLOROMETHANE } \\
\text { CHLOROMETHANE } \\
\text { CHLOROMETHANE } \\
\text { CHROMIUM } \\
\text { CHROMIUM } \\
\text { CHROMIUM } \\
\text { CHROMIUM } \\
\text { CHROMIUM } \\
\text { CHROMIUM } \\
\text { CHROMIUM } \\
\text { CHROMIUM } \\
\text { CHRYSENE } \\
\text { CHENG }\end{array}$ & $\begin{array}{l}\text { ND } \\
\text { ND } \\
\text { ND } \\
\text { ND } \\
10600 \\
97500 \\
88800 \\
10700 \\
11200 \\
11100 \\
11700 \\
11200 \\
\text { ND } \\
\text { ND } \\
\text { ND } \\
\text { ND } \\
\text { ND } \\
\text { ND } \\
\text { ND } \\
\text { ND } \\
\text { ND } \\
\text { ND } \\
\text { ND } \\
8.39 \\
\text { ND } \\
\text { (3.4) } \\
\text { ND } \\
\text { ND } \\
9.99 \\
9.97 \\
9.87 \\
9.87 \\
8.70 \\
8.68 \\
8.64 \\
9.76 \\
\text { ND } \\
\text { ND } \\
\text { ND } \\
\text { ND } \\
\text { ND } \\
\text { ND } \\
\text { ND } \\
\text { ND } \\
\text { ND } \\
\text { ND } \\
\text { ND } \\
\text { ND } \\
\text { ND } \\
\text { ND } \\
\text { ND } \\
\text { ND } \\
1.2 \\
1.4 \\
1.3 \\
1.6 \\
\text { ND } \\
\text { ND } \\
\text { ND } \\
\text { ND } \\
\text { ND }\end{array}$ & 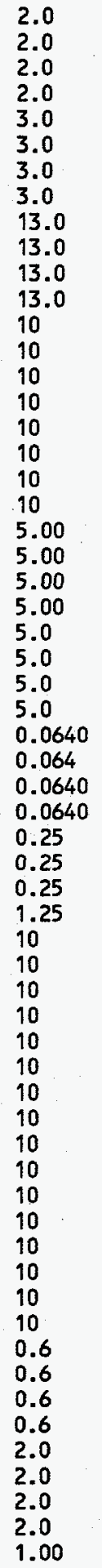 & 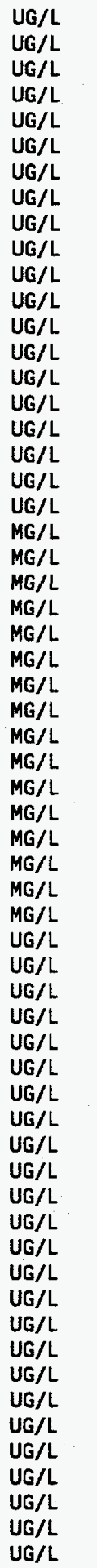 & 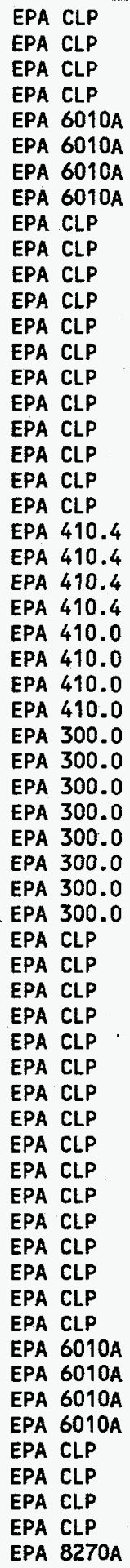 \\
\hline
\end{tabular}




\begin{tabular}{|c|c|c|c|c|c|c|c|}
\hline WSSRAP_ID & DATE_SAM & PARAMETER & CONC & $\mathrm{DL}$ & UNITS & METHOD & \\
\hline 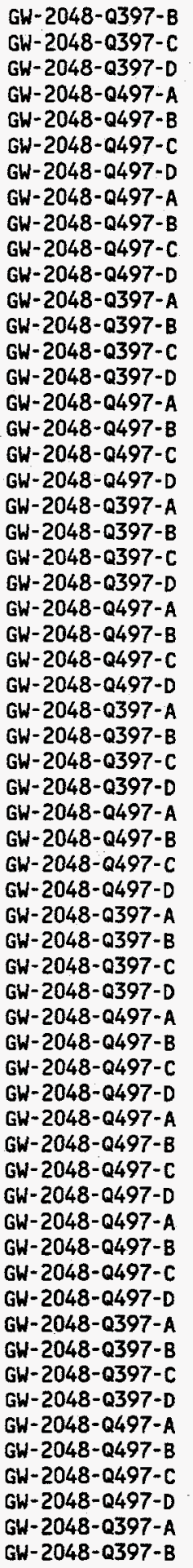 & $\begin{array}{l}07 / 01 / 97 \\
07 / 01 / 97 \\
07 / 01 / 97 \\
10 / 01 / 97 \\
10 / 01 / 97 \\
10 / 01 / 97 \\
10 / 01 / 97 \\
10 / 01 / 97 \\
10 / 01 / 97 \\
10 / 01 / 97 \\
10 / 01 / 97 \\
07 / 01 / 97 \\
07 / 01 / 97 \\
07 / 01 / 97 \\
07 / 01 / 97 \\
10 / 01 / 97 \\
10 / 01 / 97 \\
10 / 01 / 97 \\
10 / 01 / 97 \\
07 / 01 / 97 \\
07 / 01 / 97 \\
07 / 01 / 97 \\
07 / 01 / 97 \\
10 / 01 / 97 \\
10 / 01 / 97 \\
10 / 01 / 97 \\
10 / 01 / 97 \\
07 / 01 / 97 \\
07 / 01 / 97 \\
07 / 01 / 97 \\
07 / 01 / 97 \\
10 / 01 / 97 \\
10 / 01 / 97 \\
10 / 01 / 97 \\
10 / 01 / 97 \\
07 / 01 / 97 \\
07 / 01 / 97 \\
07 / 01 / 97 \\
07 / 01 / 97 \\
10 / 01 / 97 \\
10 / 01 / 97 \\
10 / 01 / 97 \\
10 / 01 / 97 \\
10 / 01 / 97 \\
10 / 01 / 97 \\
10 / 01 / 97 \\
10 / 01 / 97 \\
10 / 01 / 97 \\
10 / 01 / 97 \\
10 / 01 / 97 \\
10 / 01 / 97 \\
07 / 01 / 97 \\
07 / 01 / 97 \\
07 / 01 / 97 \\
07 / 01 / 97 \\
10 / 01 / 97 \\
10 / 01 / 97 \\
10 / 01 / 97 \\
10 / 01 / 97 \\
07 / 01 / 97 \\
07 / 01 / 97\end{array}$ & 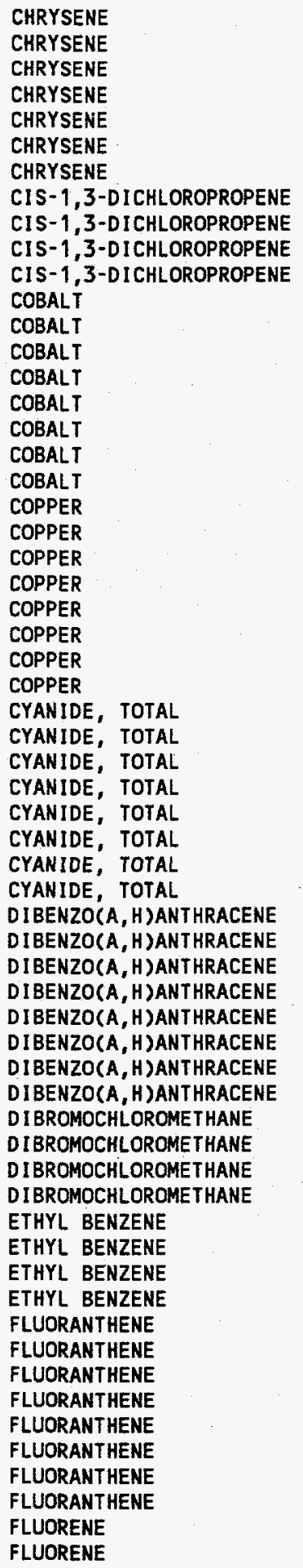 & $\begin{array}{l}\text { ND } \\
\text { ND } \\
\text { ND } \\
\text { ND } \\
\text { ND } \\
\text { ND } \\
\text { ND } \\
\text { ND } \\
\text { ND } \\
\text { ND } \\
\text { ND } \\
\text { ND } \\
\text { ND } \\
\text { ND } \\
\text { ND } \\
\text { ND } \\
\text { ND } \\
\text { ND } \\
\text { ND } \\
\text { ND } \\
\text { ND } \\
\text { ND } \\
\text { ND } \\
\text { ND } \\
\text { ND } \\
\text { ND } \\
\text { ND } \\
\text { ND } \\
\text { ND } \\
\text { ND } \\
\text { ND } \\
\text { ND } \\
\text { ND } \\
\text { ND } \\
\text { ND } \\
\text { ND } \\
\text { ND } \\
\text { ND } \\
\text { ND } \\
\text { ND } \\
\text { ND } \\
\text { ND } \\
\text { ND } \\
\text { ND } \\
\text { ND } \\
\text { ND } \\
\text { ND } \\
\text { ND } \\
\text { ND } \\
\text { ND } \\
\text { ND } \\
\text { ND } \\
\text { ND } \\
\text { ND } \\
\text { ND } \\
\text { ND } \\
\text { ND } \\
\text { ND } \\
\text { ND } \\
\text { ND }\end{array}$ & $\begin{array}{l}1.00 \\
1.00 \\
1.00 \\
0.5 \\
0.5 \\
0.5 \\
0.5 \\
10 \\
10 \\
10 \\
10 \\
0.6 \\
0.6 \\
0.6 \\
0.6 \\
2.0 \\
2.0 \\
42.0 \\
2.0 \\
0.6 \\
0.6 \\
0.6 \\
0.6 \\
2.0 \\
2.0 \\
2.0 \\
2.0 \\
2.10 \\
2.10 \\
2.10 \\
2.10 \\
4.0 \\
4.0 \\
4.0 \\
4.0 \\
1.00 \\
1.00 \\
1.00 \\
0.5 \\
1.00 \\
0.5 \\
1.00 \\
1.00 \\
0.5 \\
0.5 \\
0.5 \\
10 \\
10 \\
10 \\
10 \\
10 \\
10 \\
10 \\
10 \\
1.00 \\
1.00 \\
1.00 \\
0\end{array}$ & 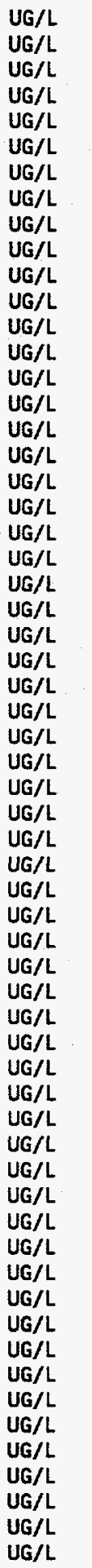 & 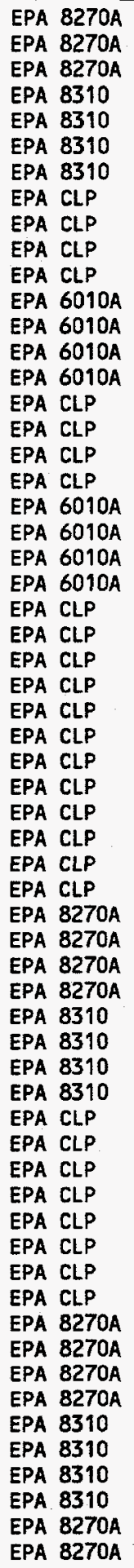 & . \\
\hline
\end{tabular}




\begin{tabular}{|c|c|c|c|c|c|c|c|}
\hline WSSRAP_ID & DATE_SAM & PARAMETER & CONC & $\mathrm{DL}$ & UNITS & METHOD & \\
\hline 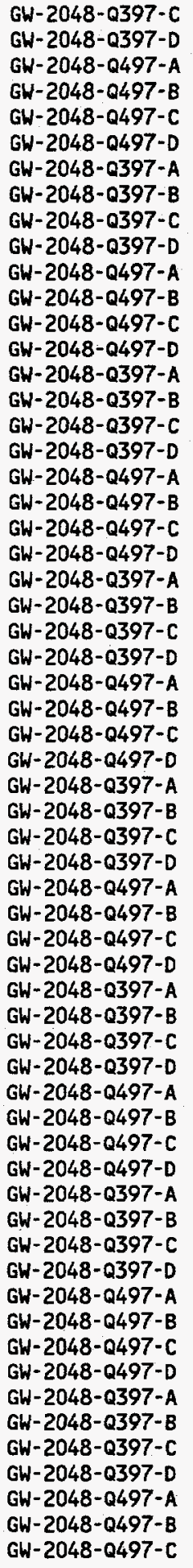 & $\begin{array}{l}07 / 01 / 97 \\
07 / 01 / 97 \\
10 / 01 / 97 \\
10 / 01 / 97 \\
10 / 01 / 97 \\
10 / 01 / 97 \\
07 / 01 / 97 \\
07 / 01 / 97 \\
07 / 01 / 97 \\
07 / 01 / 97 \\
10 / 01 / 97 \\
10 / 01 / 97 \\
10 / 01 / 97 \\
10 / 01 / 97 \\
07 / 01 / 97 \\
07 / 01 / 97 \\
07 / 01 / 97 \\
07 / 01 / 97 \\
10 / 01 / 97 \\
10 / 01 / 97 \\
10 / 01 / 97 \\
10 / 01 / 97 \\
07 / 01 / 97 \\
07 / 01 / 97 \\
07 / 01 / 97 \\
07 / 01 / 97 \\
10 / 01 / 97 \\
10 / 01 / 97 \\
10 / 01 / 97 \\
10 / 01 / 97 \\
07 / 01 / 97 \\
07 / 01 / 97 \\
07 / 01 / 97 \\
07 / 01 / 97 \\
10 / 01 / 97 \\
10 / 01 / 97 \\
10 / 01 / 97 \\
10 / 01 / 97 \\
07 / 01 / 97 \\
07 / 01 / 97 \\
07 / 01 / 97 \\
07 / 01 / 97 \\
10 / 01 / 97 \\
10 / 01 / 97 \\
10 / 01 / 97 \\
10 / 01 / 97 \\
07 / 01 / 97 \\
07 / 01 / 97 \\
07 / 01 / 97 \\
07 / 01 / 97 \\
10 / 01 / 97 \\
10 / 01 / 97 \\
10 / 01 / 97 \\
10 / 01 / 97 \\
07 / 01 / 97 \\
07 / 01 / 97 \\
07 / 01 / 97 \\
07 / 01 / 97 \\
10 / 01 / 97 \\
10 / 01 / 97 \\
10 / 01 / 97\end{array}$ & 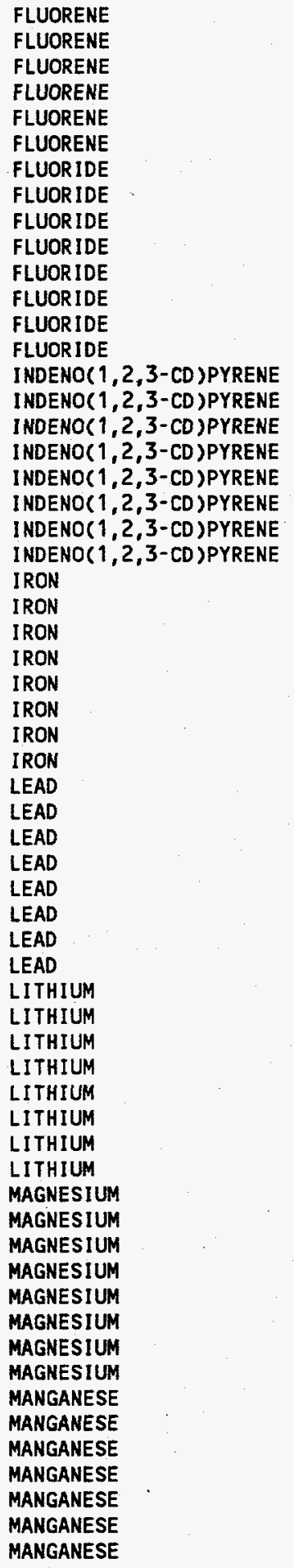 & $\begin{array}{l}\text { ND } \\
\text { ND } \\
\text { ND } \\
\text { ND } \\
\text { ND } \\
\text { ND } \\
0.157 \\
0.152 \\
0.155 \\
0.161 \\
0.14 \\
0.14 \\
0.14 \\
0.14 \\
\text { ND } \\
\text { ND } \\
\text { ND } \\
\text { ND } \\
\text { ND } \\
\text { ND } \\
\text { ND } \\
\text { ND } \\
13.3 \\
40.6 \\
7.9 \\
9.7 \\
177 \\
191 \\
195 \\
187 \\
\text { ND } \\
\text { ND } \\
\text { ND } \\
\text { ND } \\
\text { ND } \\
\text { ND } \\
\text { ND } \\
\text { ND } \\
\text { ND } \\
16.7 \\
12.6 \\
\text { ND } \\
7.3 \\
7.2 \\
7.5 \\
7.0 \\
35800 \\
32700 \\
29700 \\
35900 \\
38600 \\
38100 \\
39700 \\
38200 \\
4.6 \\
3.2 \\
2.4 \\
2.5 \\
5.6 \\
4.2 \\
3.5\end{array}$ & 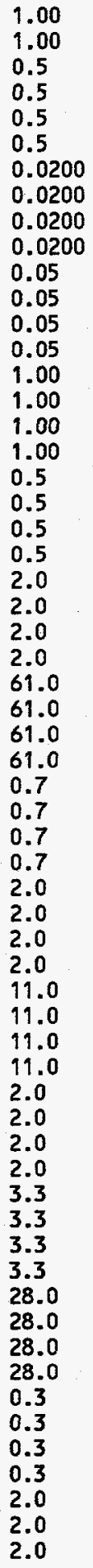 & 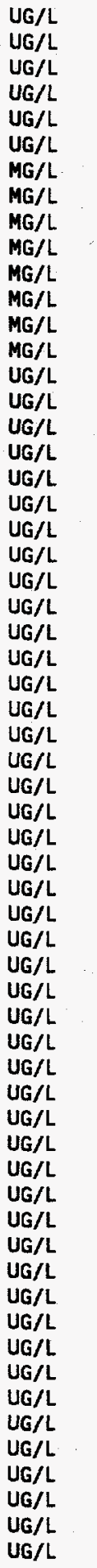 & 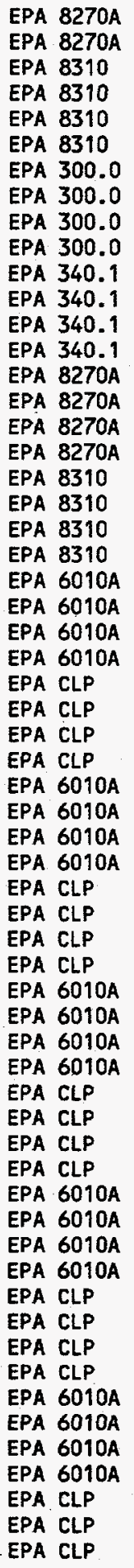 & - \\
\hline
\end{tabular}




\begin{tabular}{|c|c|c|c|c|c|c|}
\hline WSSRAP_ID & DATE_SAM & PARAMETER & CONC & $\mathrm{DL}$ & UNITS & METHOD \\
\hline 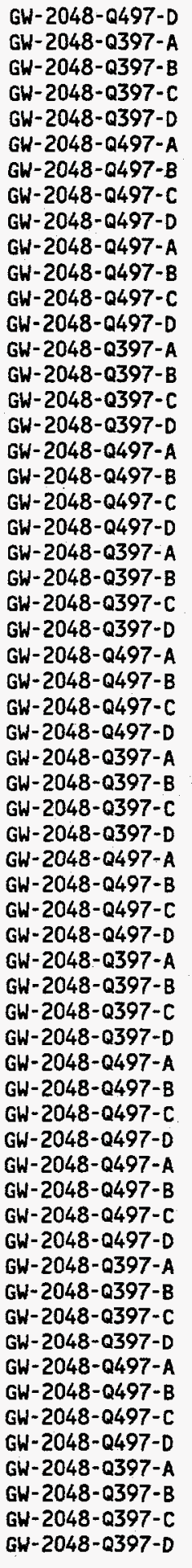 & $\begin{array}{l}10 / 01 / 97 \\
07 / 01 / 97 \\
07 / 01 / 97 \\
07 / 01 / 97 \\
07 / 01 / 97 \\
10 / 01 / 97 \\
10 / 01 / 97 \\
10 / 01 / 97 \\
10 / 01 / 97 \\
10 / 01 / 97 \\
10 / 01 / 97 \\
10 / 01 / 97 \\
10 / 01 / 97 \\
07 / 01 / 97 \\
07 / 01 / 97 \\
07 / 01 / 97 \\
07 / 01 / 97 \\
10 / 01 / 97 \\
10 / 01 / 97 \\
10 / 01 / 97 \\
10 / 01 / 97 \\
07 / 01 / 97 \\
07 / 01 / 97 \\
07 / 01 / 97 \\
07 / 01 / 97 \\
10 / 01 / 97 \\
10 / 01 / 97 \\
10 / 01 / 97 \\
10 / 01 / 97 \\
07 / 01 / 97 \\
07 / 01 / 97 \\
07 / 01 / 97 \\
07 / 01 / 97 \\
10 / 01 / 97 \\
10 / 01 / 97 \\
10 / 01 / 97 \\
10 / 01 / 97 \\
07 / 01 / 97 \\
07 / 01 / 97 \\
07 / 01 / 97 \\
07 / 01 / 97 \\
10 / 01 / 97 \\
10 / 01 / 97 \\
10 / 01 / 97 \\
10 / 01 / 97 \\
10 / 01 / 97 \\
10 / 01 / 97 \\
10 / 01 / 97 \\
10 / 01 / 97 \\
07 / 01 / 97 \\
07 / 01 / 97 \\
07 / 011 / 97 \\
07 / 01 / 97 \\
10 / 01 / 97 \\
10 / 01 / 97 \\
10 / 011 / 97 \\
10 / 01 / 97 \\
07 / 01 / 97 \\
07 / 01 / 97 \\
07 / 01 / 97 \\
07 / 01 / 97\end{array}$ & $\begin{array}{l}\text { MANGANESE } \\
\text { MERCURY } \\
\text { MERCURY } \\
\text { MERCURY } \\
\text { MERCURY } \\
\text { MERCURY } \\
\text { MERCURY } \\
\text { MERCURY } \\
\text { MERCURY } \\
\text { METHYLENE CHLORIDE } \\
\text { METHYLENE CHLORIDE } \\
\text { METHYLENE CHLORIDE } \\
\text { METHYLENE CHLORIDE } \\
\text { MOLYBDENUM } \\
\text { MOLYBDENUM } \\
\text { MOLYBDENUM } \\
\text { MOLYBDENUM } \\
\text { MOLYBDENUM } \\
\text { MOLYBDENUM } \\
\text { MOLYBDENUM } \\
\text { MOLYBDENUM } \\
\text { NAPHTHALENE } \\
\text { NAPHTHALENE } \\
\text { NAPHTHALENE } \\
\text { NAPHTHALENE } \\
\text { NAPHTHALENE } \\
\text { NAPHTHALENE } \\
\text { NAPHTHALENE } \\
\text { NAPHTHALENE } \\
\text { NICKEL } \\
\text { NICKEL } \\
\text { NICKEL } \\
\text { NICKEL } \\
\text { NICKEL } \\
\text { NICKEL } \\
\text { NICKEL } \\
\text { NICKEL } \\
\text { NI TRATE-N } \\
\text { NI TRATE-N } \\
\text { NITRATE-N } \\
\text { NITRATE-N } \\
\text { NI TRATE-N } \\
\text { NITRATE-N } \\
\text { NITRATE-N } \\
\text { NITRATE-N } \\
\text { NI TROBENZENE } \\
\text { NI TROBENZENE } \\
\text { NITROBENZENE } \\
\text { NITROBENZENE } \\
\text { PHENANTHRENE } \\
\text { PHENANTHRENE } \\
\text { PHENANTHRENE } \\
\text { PHENANTHRENE } \\
\text { PHENANTHRENE } \\
\text { PHENANTHRENE } \\
\text { PHENANTHRENE } \\
\text { PHENANTHRENE } \\
\text { POTASSIUM } \\
\text { POTASSIUM } \\
\text { POTASSIUM } \\
\text { POTASSIUM } \\
\end{array}$ & $\begin{array}{l}3.0 \\
0.42 \\
0.19 \\
0.18 \\
0.18 \\
\text { ND } \\
\text { ND } \\
\text { ND } \\
\text { ND } \\
\text { ND } \\
\text { ND } \\
39 \\
\text { ND } \\
1.4 \\
0.87 \\
1.1 \\
0.94 \\
\text { ND } \\
\text { ND } \\
\text { ND } \\
\text { ND } \\
\text { ND } \\
\text { ND } \\
\text { ND } \\
\text { ND } \\
\text { ND } \\
\text { ND } \\
\text { ND } \\
\text { ND } \\
1.7 \\
1.5 \\
1.3 \\
1.5 \\
\text { ND } \\
\text { ND } \\
\text { ND } \\
\text { ND } \\
0.830 \\
0.850 \\
0.840 \\
0.850 \\
1.11 \\
1.06 \\
1.14 \\
1.17 \\
\text { ND } \\
\text { ND } \\
\text { ND } \\
\text { ND } \\
\text { ND } \\
\text { ND } \\
\text { ND } \\
\text { ND } \\
\text { ND } \\
\text { ND } \\
\text { ND } \\
\text { ND } \\
3020 \\
2800 \\
2540 \\
2910 \\
\end{array}$ & $\begin{array}{l}2.0 \\
0.1 \\
0.1 \\
0.1 \\
0.1 \\
0.10 \\
0.10 \\
0.10 \\
0.10 \\
10 \\
10 \\
10 \\
10 \\
0.7 \\
0.7 \\
0.7 \\
0.7 \\
7.0 \\
7.0 \\
7.0 \\
7.0 \\
1.00 \\
1.00 \\
1.00 \\
1.00 \\
0.5 \\
0.5 \\
0.5 \\
0.5 \\
1.0 \\
1.0 \\
1.0 \\
1.0 \\
2.0 \\
2.0 \\
2.0 \\
2.0 \\
0.00700 \\
0.00700 \\
0.00700 \\
0.00700 \\
0.040 \\
0.020 \\
0.040 \\
0.040 \\
0.030 \\
0.030 \\
0.030 \\
0.030 \\
1.00 \\
1.00 \\
1.00 \\
1.00 \\
0.5 \\
0.5 \\
0.5 \\
0.5 \\
11.0 \\
11.0 \\
11.0 \\
11.0 \\
\\
\\
\end{array}$ & 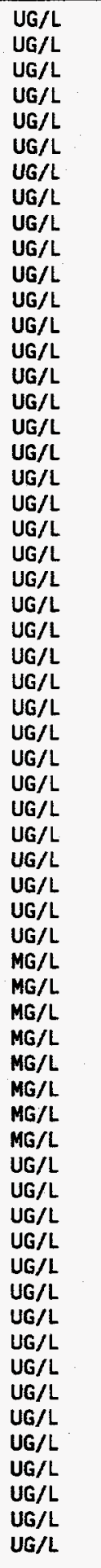 & $\begin{array}{l}\text { EPA CLP } \\
\text { EPA } 7470 A \\
\text { EPA } 7470 A \\
\text { EPA } 7470 A \\
\text { EPA } 7470 A \\
\text { EPA CLP } \\
\text { EPA CLP } \\
\text { EPA CLP } \\
\text { EPA CLP } \\
\text { EPA CLP } \\
\text { EPA CLP } \\
\text { EPA CLP } \\
\text { EPA CLP } \\
\text { EPA } 6010 A \\
\text { EPA } 6010 A \\
\text { EPA } 6010 A \\
\text { EPA } 6010 A \\
\text { EPA CLP } \\
\text { EPA CLP } \\
\text { EPA CLP } \\
\text { EPA CLP } \\
\text { EPA } 8270 A \\
\text { EPA } 8270 A \\
\text { EPA } 8270 A \\
\text { EPA } 8270 A \\
\text { EPA } 8310 \\
\text { EPA } 8310 \\
\text { EPA } 8310 \\
\text { EPA } 8310 \\
\text { EPA } 6010 A \\
\text { EPA } 6010 A \\
\text { EPA } 6010 A \\
\text { EPA } 6010 A \\
\text { EPA CLP } \\
\text { EPA CLP } \\
\text { EPA CLP } \\
\text { EPA CLP } \\
\text { EPA } 353.1 \\
\text { EPA } 353.1 \\
\text { EPA } 353.1 \\
\text { EPA } 353.1 \\
\text { EPA } 353.1 \\
\text { EPA } 353.1 \\
\text { EPA } 353.1 \\
\text { EPA } 353.1 \\
\text { USATHAMA } \\
\text { USATHAMA } \\
\text { USATHAMA } \\
\text { USATHAMA } \\
\text { EPA } 8270 A \\
\text { EPA } 8270 A \\
\text { EPA } 8270 A \\
\text { EPA } 8270 A \\
\text { EPA } 8310 \\
\text { EPA } 831010 \\
\text { EPA } 8310 \\
\text { EPA } 8310 \\
\text { EPA } 6010 A \\
\text { EPA } \\
\text { E010A } \\
\text { EA }\end{array}$ \\
\hline
\end{tabular}




\begin{tabular}{|c|c|c|c|c|c|c|}
\hline WSSRAP_ID & DATE_SAM & PARAMETER & CONC & $\mathrm{DL}$ & UNI TS & METHOD \\
\hline 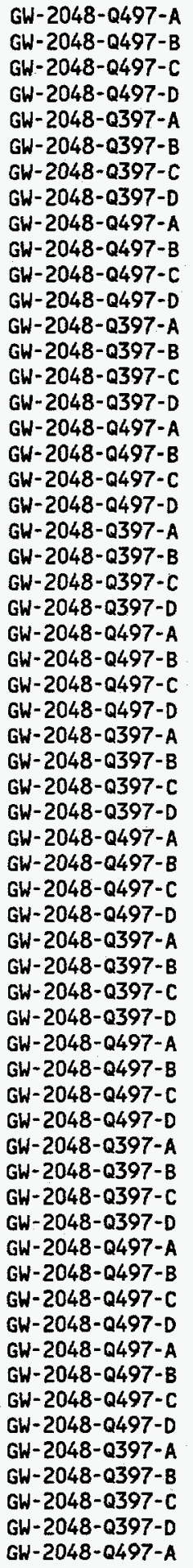 & $\begin{array}{l}10 / 01 / 97 \\
10 / 01 / 97 \\
10 / 01 / 97 \\
10 / 01 / 97 \\
07 / 01 / 97 \\
07 / 01 / 97 \\
07 / 01 / 97 \\
07 / 01 / 97 \\
10 / 01 / 97 \\
10 / 01 / 97 \\
10 / 01 / 97 \\
10 / 01 / 97 \\
07 / 01 / 97 \\
07 / 01 / 97 \\
07 / 01 / 97 \\
07 / 01 / 97 \\
10 / 01 / 97 \\
10 / 01 / 97 \\
10 / 01 / 97 \\
10 / 01 / 97 \\
07 / 01 / 97 \\
07 / 01 / 97 \\
07 / 01 / 97 \\
07 / 01 / 97 \\
10 / 01 / 97 \\
10 / 01 / 97 \\
10 / 01 / 97 \\
10 / 01 / 97 \\
07 / 01 / 97 \\
07 / 01 / 97 \\
07 / 01 / 97 \\
07 / 01 / 97 \\
10 / 01 / 97 \\
10 / 01 / 97 \\
10 / 01 / 97 \\
10 / 01 / 97 \\
07 / 01 / 97 \\
07 / 01 / 97 \\
07 / 01 / 97 \\
07 / 01 / 97 \\
10 / 01 / 97 \\
10 / 01 / 97 \\
10 / 01 / 97 \\
10 / 01 / 97 \\
07 / 01 / 97 \\
07 / 01 / 97 \\
07 / 01 / 97 \\
07 / 01 / 97 \\
10 / 01 / 97 \\
10 / 01 / 97 \\
10 / 01 / 97 \\
10 / 01 / 97 \\
10 / 01 / 97 \\
10 / 01 / 97 \\
10 / 01 / 97 \\
10 / 01 / 97 \\
07 / 01 / 97 \\
07 / 01 / 97 \\
07 / 01 / 97 \\
07 / 01 / 97 \\
10 / 01 / 97\end{array}$ & $\begin{array}{l}\text { POTASSIUM } \\
\text { POTASSIUM } \\
\text { POTASSIUM } \\
\text { POTASSIUM } \\
\text { PYRENE } \\
\text { PYRENE } \\
\text { PYRENE } \\
\text { PYRENE } \\
\text { PYRENE } \\
\text { PYRENE } \\
\text { PYRENE } \\
\text { PYRENE } \\
\text { RADIUM-226 } \\
\text { RADIUM-226 } \\
\text { RADIUM-226 } \\
\text { RADIUM-226 } \\
\text { RADIUM-226 } \\
\text { RADIUM-226 } \\
\text { RADIUM-226 } \\
\text { RADIUM-226 } \\
\text { RADIUM-228 } \\
\text { RADIUM-228 } \\
\text { RADIUM-228 } \\
\text { RADIIUM-228 } \\
\text { RADIUM-228 } \\
\text { RADIUM-228 } \\
\text { RADIUM-228 } \\
\text { RADIUM-228 } \\
\text { SELENIUM } \\
\text { SELENIUM } \\
\text { SELENIUM } \\
\text { SELENIUM } \\
\text { SELENIUM } \\
\text { SELENIUM } \\
\text { SELENIUM } \\
\text { SELENIUM } \\
\text { SILVER } \\
\text { SILVER } \\
\text { SILVER } \\
\text { SILVER } \\
\text { SILVER } \\
\text { SILVER } \\
\text { SILVER } \\
\text { SILVER } \\
\text { SODIUM } \\
\text { SODIUM } \\
\text { SODIUM } \\
\text { SODIIUM } \\
\text { SODIUM } \\
\text { SODIUM } \\
\text { SODIUM } \\
\text { SODIUM } \\
\text { STYRENE } \\
\text { STYRENE } \\
\text { STYRENE } \\
\text { STYRENE } \\
\text { SULFATE } \\
\text { SULFATE } \\
\text { SULFATE } \\
\text { SULFATE } \\
\text { SULFATE }\end{array}$ & $\begin{array}{l}3370 \\
3250 \\
3330 \\
3280 \\
\text { ND } \\
\text { ND } \\
\text { ND } \\
\text { ND } \\
\text { ND } \\
\text { ND } \\
\text { ND } \\
\text { ND } \\
60.24 \\
60.03 \\
C 0.16 \\
C 0.03 \\
0.155 \\
60.05 \\
C 0.05 \\
C 0.06 \\
1.79 \\
2.12 \\
3.23 \\
1.35 \\
1.36 \\
C 0.25 \\
C 0.48 \\
\text { ND } \\
10.4 \\
10.2 \\
9.7 \\
10.1 \\
12.5 \\
11.7 \\
12.8 \\
9.4 \\
1.7 \\
2.0 \\
2.4 \\
1.8 \\
\text { ND } \\
\text { ND } \\
\text { ND } \\
\text { ND } \\
76200 \\
70600 \\
63500 \\
75300 \\
75000 \\
74600 \\
79400 \\
74800 \\
\text { ND } \\
\text { ND } \\
\text { ND } \\
\text { ND } \\
183 \\
181 \\
178 \\
177 \\
200\end{array}$ & 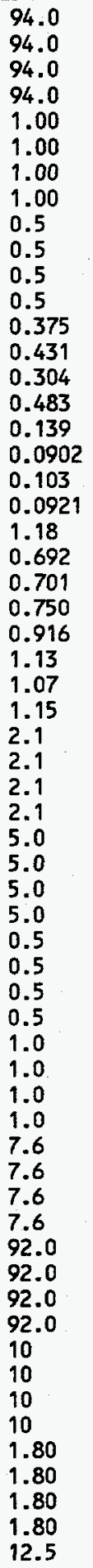 & $\begin{array}{l}U G / L \\
U G / L \\
U G / L \\
U G / L \\
U G / L \\
U G / L \\
U G / L \\
U G / L \\
U G / L \\
U G / L \\
U G / L \\
U G / L \\
P C I / L \\
P C I / L \\
P C I / L \\
P C I / L \\
P C I / L \\
P C I / L \\
P C I / L \\
P C I / L \\
P C I / L \\
P C I / L \\
P C I / L \\
P C I / L \\
P C I / L \\
P C I / L \\
P C I / L \\
P C I / L \\
U G / L \\
U G / L \\
U G / L \\
U G / L \\
U G / L \\
U G / L \\
U G / L \\
U G / L \\
U G / L \\
U G / L \\
U G / L \\
U G / L \\
U G / L \\
U G / L \\
U G / L \\
U G / L \\
U G / L \\
U G / L \\
U G / L \\
U G / L \\
U G / L \\
U G / L \\
U G / L \\
U G / L \\
U G / L \\
U G / L \\
U G / L \\
U G / L \\
M G / L \\
M G / L \\
M G / L \\
M G / L \\
\text { UG } / L \\
\end{array}$ & 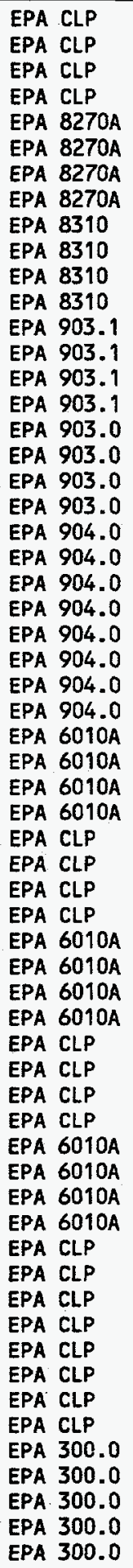 \\
\hline
\end{tabular}




\begin{tabular}{|c|c|c|c|c|c|c|}
\hline WSSRAP_ID & DATE_SAM & PARAMETER & CONC & DL & UNITS & METHOD \\
\hline 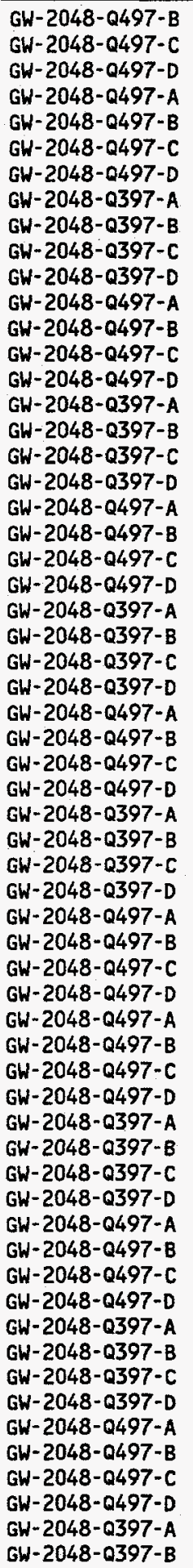 & $\begin{array}{l}10 / 01 / 97 \\
10 / 01 / 97 \\
10 / 01 / 97 \\
10 / 01 / 97 \\
10 / 01 / 97 \\
10 / 01 / 97 \\
10 / 01 / 97 \\
07 / 01 / 97 \\
07 / 01 / 97 \\
07 / 01 / 97 \\
07 / 01 / 97 \\
10 / 01 / 97 \\
10 / 01 / 97 \\
10 / 01 / 97 \\
10 / 01 / 97 \\
07 / 01 / 97 \\
07 / 01 / 97 \\
07 / 01 / 97 \\
07 / 01 / 97 \\
10 / 01 / 97 \\
10 / 01 / 97 \\
10 / 01 / 97 \\
10 / 01 / 97 \\
07 / 01 / 97 \\
07 / 01 / 97 \\
07 / 01 / 97 \\
07 / 01 / 97 \\
10 / 01 / 97 \\
10 / 01 / 97 \\
10 / 01 / 97 \\
10 / 01 / 97 \\
07 / 01 / 97 \\
07 / 01 / 97 \\
07 / 01 / 97 \\
07 / 01 / 97 \\
10 / 01 / 97 \\
10 / 01 / 97 \\
10 / 01 / 97 \\
10 / 01 / 97 \\
10 / 01 / 97 \\
10 / 01 / 97 \\
10 / 01 / 97 \\
10 / 01 / 97 \\
07 / 01 / 97 \\
07 / 01 / 97 \\
07 / 01 / 97 \\
07 / 01 / 97 \\
10 / 01 / 97 \\
10 / 01 / 97 \\
10 / 01 / 97 \\
10 / 01 / 97 \\
07 / 01 / 97 \\
07 / 01 / 97 \\
07 / 01 / 97 \\
07 / 01 / 97 \\
10 / 01 / 97 \\
10 / 01 / 97 \\
10 / 01 / 97 \\
10 / 01 / 97 \\
07 / 01 / 97 \\
07 / 01 / 97\end{array}$ & $\begin{array}{l}\text { SULFATE } \\
\text { SULFATE } \\
\text { SULFATE } \\
\text { TETRACHLOROETHENE } \\
\text { TETRACHLOROETHENE } \\
\text { TETRACHLOROETHENE } \\
\text { TETRACHLOROETHENE } \\
\text { THALLIUM } \\
\text { THALLIUM } \\
\text { THALLIUM } \\
\text { THALLIUM } \\
\text { THALLIUM } \\
\text { THALLIUM } \\
\text { THALLIUM } \\
\text { THALLIUM } \\
\text { THORIUM-228 } \\
\text { THORIUM-228 } \\
\text { THORIUM-228 } \\
\text { THORIUM-228 } \\
\text { THORIUM-228 } \\
\text { THORIUM-228 } \\
\text { THORIUM-228 } \\
\text { THORIUM-228 } \\
\text { THORIUM-230 } \\
\text { THORIUM-230 } \\
\text { THORIUM-230 } \\
\text { THORIUM-230 } \\
\text { THORIUM-230 } \\
\text { THORIUM-230 } \\
\text { THORIUM-230 } \\
\text { THORIUM-230 } \\
\text { THORIUM-232 } \\
\text { THORIUM-232 } \\
\text { THORIUM-232 } \\
\text { THORIUM-232 } \\
\text { THORIUM-232 } \\
\text { THORIUM-232 } \\
\text { THORIUM-232 } \\
\text { THORIUM-232 } \\
\text { TOLUENE } \\
\text { TOLUENE } \\
\text { TOLUENE } \\
\text { TOLUENE } \\
\text { TOTAL DISSOLVED SOLIDS } \\
\text { TOTAL DISSOLVED SOLIDS } \\
\text { TOTAL DISSOLVED SOLIDS } \\
\text { TOTAL DISSOLVED SOLIDS } \\
\text { TOTAL DISSOLVED SOLIDS } \\
\text { TOTAL DISSOLVED SOLIDS } \\
\text { TOTAL DISSOLVED SOLIDS } \\
\text { TOTAL DISSOLVED SOLIDS } \\
\text { TOTAL ORGANIC CARBON } \\
\text { TOTAL ORGANIC CARBON } \\
\text { TOTAL ORGANIC CARBON } \\
\text { TOTAL ORGANIC CARBON } \\
\text { TOTAL ORGANIC CARBON } \\
\text { TOTAL ORGANIC CARBON } \\
\text { TOTAL ORGANIC CARBON } \\
\text { TOTAL ORGANIC CARBON } \\
\text { TOX } \\
\text { TOX }\end{array}$ & $\begin{array}{l}193 \\
193 \\
192 \\
\text { ND } \\
\text { ND } \\
\text { ND } \\
\text { ND } \\
\text { ND } \\
4.2 \\
3.5 \\
\text { ND } \\
\text { ND } \\
\text { ND } \\
\text { ND } \\
\text { ND } \\
\text { ND } \\
\text { ND } \\
\text { ND } \\
C 0.00 \\
C 0.09 \\
10.10 \\
<0.10 \\
0.140 \\
0.265 \\
0.079 \\
0.120 \\
0.158 \\
C 0.24 \\
0.324 \\
C 0.21 \\
60.13 \\
C 0.00 \\
60.01 \\
\text { ND } \\
60.01 \\
60.09 \\
60.10 \\
\text { ND } \\
C 0.04 \\
\text { ND } \\
\text { ND } \\
\text { ND } \\
\text { ND } \\
658 \\
640 \\
651 \\
637 \\
826 \\
848 \\
840 \\
904 \\
3.20 \\
3.20 \\
3.42 \\
3.22 \\
10.6 \\
24.4 \\
26.4 \\
29.5 \\
1.17 \\
0.030\end{array}$ & 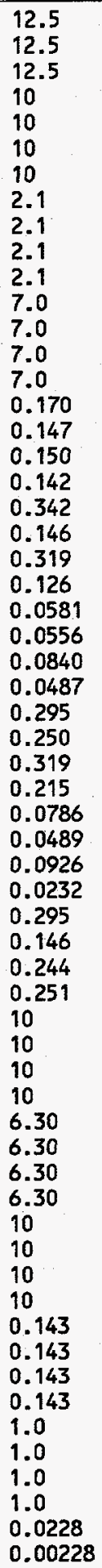 & $\begin{array}{l}M G / L \\
M G / L \\
M G / L \\
U G / L \\
U G / L \\
U G / L \\
U G / L \\
U G / L \\
U G / L \\
U G / L \\
U G / L \\
U G / L \\
U G / L \\
U G / L \\
U G / L \\
P C I / L \\
P C I / L \\
P C I / L \\
P C I / L \\
P C I / L \\
P C I / L \\
P C I / L \\
P C I / L \\
P C I / L \\
P C I / L \\
P C I / L \\
P C I / L \\
P C I / L \\
P C I / L \\
P C I / L \\
P C I / L \\
P C I / L \\
P C I / L \\
P C I / L \\
P C I / L \\
P C I / L \\
P C I / L \\
P C I / L \\
P C I / L \\
U G / L \\
U G / L \\
U G / L \\
U G / L \\
M G / L \\
M G / L \\
M G / L \\
M G / L \\
M G / L \\
M G / L \\
M G / L \\
M G / L \\
M G / L \\
M G / L \\
M G / L \\
M G / L \\
M G / L \\
M G / L \\
M G / L \\
M G / L \\
M G / L \\
M G / L \\
\end{array}$ & $\begin{array}{l}\text { EPA } 300.0 \\
\text { EPA } 300.0 \\
\text { EPA } 300.0 \\
\text { EPA CLP } \\
\text { EPA CLP } \\
\text { EPA CLP } \\
\text { EPA CLP } \\
\text { EPA } 6010 A \\
\text { EPA } 6010 A \\
\text { EPA } 6010 A \\
\text { EPA } 6010 A \\
\text { EPA CLP } \\
\text { EPA CLP } \\
\text { EPA CLP } \\
\text { EPA CLP } \\
\text { HASL } 300 \\
\text { HASL } 300 \\
\text { HASL } 300 \\
\text { HASL } 300 \\
\text { EML TH-01 } \\
\text { EML TH-01 } \\
\text { EML TH-01 } \\
\text { EML TH-01 } \\
\text { HASL } 300 \\
\text { HASL } 300 \\
\text { HASL } 300 \\
\text { HASL } 300 \\
\text { EML TH-01 } \\
\text { EML TH-01 } \\
\text { EML TH-01 } \\
\text { EML TH-01 } \\
\text { HASL } 300 \\
\text { HASL } 300 \\
\text { HASL } 300 \\
\text { HASL } 300 \\
\text { EML TH-01 } \\
\text { EML TH-01 } \\
\text { EML TH-01 } \\
\text { EML TH-01 } \\
\text { EPA CLP } \\
\text { EPA CLP } \\
\text { EPA CLP } \\
\text { EPA CLP } \\
\text { EPA } 160.1 \\
\text { EPA } 160.1 \\
\text { EPA } 160.1 \\
\text { EPA } 160.1 \\
\text { EPA } 160.1 \\
\text { EPA } 160.1 \\
\text { EPA } 160.1 \\
\text { EPA } 160.1 \\
\text { EPA } 415.1 \\
\text { EPA } 415.1 \\
\text { EPA } 415.1 \\
\text { EPA } 415.1 \\
\text { EPA } 415.1 \\
\text { EPA } 415.1 \\
\text { EPA } 415.1 \\
\text { EPA } 415.1 \\
\text { EPA } 9020 B \\
9020 B\end{array}$ \\
\hline
\end{tabular}




\begin{tabular}{|c|c|c|c|c|c|c|}
\hline WSSRAP_ID & DATE_SAM & PARAMETER & CONC & $\mathrm{DL}$ & UNITS & METHOD \\
\hline $\begin{array}{l}\text { GW-2048-Q397-C } \\
\text { GW-2048-Q397-D } \\
\text { GW-2048-Q397-A- } \\
\text { GW-2048-Q497-A } \\
\text { GW-2048-Q497-B } \\
\text { GW-2048-Q497-C } \\
\text { GW-2048-Q497-D } \\
\text { GW-2048-Q497-A } \\
\text { GW-2048-Q497-B } \\
\text { GW-2048-Q497-C } \\
\text { GW-2048-Q497-D } \\
\text { GW-2048-Q497-A } \\
\text { GW-2048-0497-B } \\
\text { GW-2048-Q497-C } \\
\text { GW-2048-Q497-D } \\
\text { GW-2048-Q397-A } \\
\text { GW-2048-Q397-B } \\
\text { GW-2048-Q397-C } \\
\text { GW-2048-Q397-D } \\
\text { GW-2048-Q497-A } \\
\text { GW-2048-Q497-B } \\
\text { GW-2048-Q497-C } \\
\text { GW-2048-Q497-D } \\
\text { GW-2048-Q397-A } \\
\text { GW-2048-Q397-B } \\
\text { GW-2048-Q397-C } \\
\text { GW-2048-Q397-D } \\
\text { GW-2048-Q497-A } \\
\text { GW-2048-Q497-B } \\
\text { GW-2048-Q497-C } \\
\text { GW-2048-Q497-D } \\
\text { GW-2048-Q497-A } \\
\text { GW-2048-Q497-B } \\
\text { GW-2048-Q497-C } \\
\text { GW-2048-Q497-D } \\
\text { GW-2048-0497-A } \\
\text { GW-2048-Q497-B } \\
\text { GW-2048-Q497-C } \\
\text { GW-2048-Q497-D } \\
\text { GW-2048-Q397-A } \\
\text { GW-2048-Q397-B } \\
\text { GW-2048-Q397-C } \\
\text { GW-2048-Q397-D } \\
\text { GW-2048-Q497-A } \\
\text { GW-2048-Q497-B } \\
\text { GW-2048-Q497-C } \\
\text { GW-2048-Q497-D } \\
\text { GW-3003-1097 } \\
\text { GW-3003-1097 } \\
\text { GW-3003-1097 } \\
\text { GW-3003-1097 } \\
\text { GW-3003-1097 } \\
\text { GW-3003-1097 } \\
\text { GW-3003-1097 } \\
\text { GW-3003-1097 } \\
\text { GW-3003-1097 } \\
\text { GW-3003-1097 } \\
\text { GW-3023-1097 } \\
\text { GW-3023-1097 } \\
\text { GW-3023-1097 } \\
\text { GW-3023-1097 }\end{array}$ & $\begin{array}{l}07 / 01 / 97 \\
07 / 01 / 97 \\
09 / 24 / 97 \\
10 / 01 / 97 \\
10 / 01 / 97 \\
10 / 01 / 97 \\
10 / 01 / 97 \\
10 / 01 / 97 \\
10 / 01 / 97 \\
10 / 01 / 97 \\
10 / 01 / 97 \\
10 / 01 / 97 \\
10 / 01 / 97 \\
10 / 01 / 97 \\
10 / 01 / 97 \\
07 / 01 / 97 \\
07 / 01 / 97 \\
07 / 01 / 97 \\
07 / 01 / 97 \\
10 / 01 / 97 \\
10 / 01 / 97 \\
10 / 01 / 97 \\
10 / 01 / 97 \\
07 / 01 / 97 \\
07 / 01 / 97 \\
07 / 01 / 97 \\
07 / 01 / 97 \\
10 / 01 / 97 \\
10 / 01 / 97 \\
10 / 01 / 97 \\
10 / 01 / 97 \\
10 / 01 / 97 \\
10 / 01 / 97 \\
10 / 01 / 97 \\
10 / 01 / 97 \\
10 / 01 / 97 \\
10 / 01 / 97 \\
10 / 01 / 97 \\
10 / 01 / 97 \\
07 / 01 / 97 \\
07 / 01 / 97 \\
07 / 01 / 97 \\
07 / 01 / 97 \\
10 / 011 / 97 \\
10 / 01 / 97 \\
10 / 01 / 97 \\
10 / 01 / 97 \\
10 / 06 / 97 \\
10 / 06 / 97 \\
10 / 06 / 97 \\
10 / 06 / 97 \\
10 / 06 / 97 \\
10 / 06 / 97 \\
10 / 06 / 97 \\
10 / 06 / 97 \\
10 / 06 / 97 \\
10 / 06 / 97 \\
10 / 06 / 97 \\
10 / 06 / 97 \\
10 / 06 / 97 \\
10 / 06 / 97\end{array}$ & 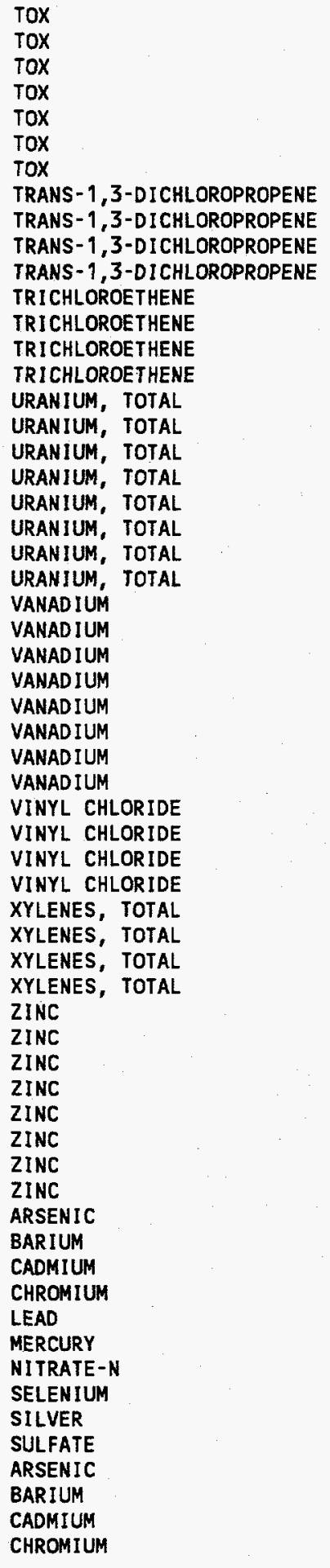 & $\begin{array}{l}0.014 \\
0.005 \\
0.031 \\
0.023 \\
0.025 \\
0.022 \\
0.013 \\
\text { ND } \\
\text { ND } \\
\text { ND } \\
\text { ND } \\
\text { ND } \\
\text { ND } \\
\text { ND } \\
\text { ND } \\
1.62 \\
1.62 \\
1.54 \\
1.47 \\
1.74 \\
1.82 \\
1.82 \\
1.61 \\
\text { ND } \\
\text { ND } \\
\text { ND } \\
\text { ND } \\
\text { ND } \\
\text { ND } \\
\text { ND } \\
\text { ND } \\
\text { ND } \\
\text { ND } \\
\text { ND } \\
\text { ND } \\
\text { ND } \\
\text { ND } \\
\text { ND } \\
\text { ND } \\
4.0 \\
10.9 \\
3.9 \\
5.7 \\
29.9 \\
24.5 \\
17.5 \\
17.0 \\
\text { ND } \\
191 \\
\text { ND } \\
\text { ND } \\
2.7 \\
\text { ND } \\
415 \\
11.3 \\
\text { ND } \\
160 \\
\text { ND } \\
\text { ND } \\
\text { ND } \\
\end{array}$ & $\begin{array}{l}0.00228 \\
0.00228 \\
0.005 \\
0.005 \\
0.005 \\
0.005 \\
0.005 \\
10 \\
10 \\
10 \\
10 \\
10 \\
10 \\
10 \\
10 \\
0.0643 \\
0.0643 \\
0.0643 \\
0.0643 \\
0.0203 \\
0.0203 \\
0.0203 \\
0.0203 \\
0.4 \\
0.4 \\
0.4 \\
0.4 \\
2.0 \\
2.0 \\
2.0 \\
2.0 \\
10 \\
10 \\
10 \\
10 \\
10 \\
10 \\
10 \\
10 \\
1.1 \\
1.1 \\
1.1 \\
1.1 \\
6.0 \\
6.0 \\
6.0 \\
6.0 \\
4.0 \\
2.0 \\
2.0 \\
2.0 \\
2.0 \\
0.10 \\
20.0 \\
5.0 \\
1.0 \\
10.0 \\
4.0 \\
2.0 \\
2.0 \\
2.0 \\
2.0 \\
\\
\\
\\
\\
\end{array}$ & $\begin{array}{l}M G / L \\
M G / L \\
M G / L \\
M G / L \\
M G / L \\
M G / L \\
M G / L \\
U G / L \\
U G / L \\
U G / L \\
U G / L \\
U G / L \\
U G / L \\
U G / L \\
U G / L \\
P C I / L \\
P C I / L \\
P C I / L \\
P C I / L \\
P C I / L \\
P C I / L \\
P C I / L \\
P C I / L \\
U G / L \\
U G / L \\
U G / L \\
U G / L \\
U G / L \\
U G / L \\
U G / L \\
U G / L \\
U G / L \\
U G / L \\
U G / L \\
U G / L \\
U G / L \\
U G / L \\
U G / L \\
U G / L \\
U G / L \\
U G / L \\
U G / L \\
U G / L \\
U G / L \\
U G / L \\
U G / L \\
U G / L \\
U G / L \\
U G / L \\
U G / L \\
U G / L \\
U G / L \\
U G / L \\
U G \\
U G \\
U G \\
U G \\
U G\end{array}$ & $\begin{array}{l}\text { EPA } 9020 B \\
\text { EPA } 9020 B \\
\text { SW846 9020A } \\
\text { EPA } 9020 A \\
\text { EPA } 9020 A \\
\text { EPA } 9020 A \\
\text { EPA } 9026 A \\
\text { EPA CLP } \\
\text { EPA CLP } \\
\text { EPA CLP } \\
\text { EPA CLP } \\
\text { EPA CLP } \\
\text { EPA CLP } \\
\text { EPA CLP } \\
\text { EPA CLP } \\
\text { ASTM } 5174-91 \\
\text { ASTM } 5174-91 \\
\text { ASTM } 5174-91 \\
\text { ASTM } 5174-91 \\
\text { ASTM } 5174-91 \\
\text { ASTM } 5174-91 \\
\text { ASTM } 5174-91 \\
\text { ASTM } 5174-91 \\
\text { EPA } 6010 A \\
\text { EPA } 6010 A \\
\text { EPA } 6010 A \\
\text { EPA } 6010 A \\
\text { EPA CLP } \\
\text { EPA CLP } \\
\text { EPA CLP } \\
\text { EPA CLP } \\
\text { EPA CLP } \\
\text { EPA CLP } \\
\text { EPA CLP } \\
\text { EPA CLP } \\
\text { EPA CLP } \\
\text { EPA CLP } \\
\text { EPA CLP } \\
\text { EPA CLP } \\
\text { EPA } 6010 A \\
\text { EPA } 6010 A \\
\text { EPA } 6010 A \\
\text { EPA } 6010 A \\
\text { EPA CLP } \\
\text { EPA CLP } \\
\text { EPA CLP } \\
\text { EPA CLP } \\
\text { EPA } 6010 A \\
\text { EPA } 6010 A \\
\text { EPA } 6010 A \\
\text { EPA } 6010 A \\
\text { EPA } 6010 A \\
\text { EPA } 7470 A \\
\text { EPA } 353.1 \\
\text { EPA } 6010 A \\
\text { EPA } 6010 A \\
\text { EPA } 300.0 \\
\text { EPA } 6010 A \\
\text { EPA } 6010 A \\
\text { EPA } 6010 A \\
\text { EPA } 6010 A \\
\end{array}$ \\
\hline
\end{tabular}




\begin{tabular}{|c|c|c|c|c|c|c|}
\hline WSSRAP_IO & DATE_SAM & PARAMETER & CONC & $D L$ & UNI TS & METHOD \\
\hline 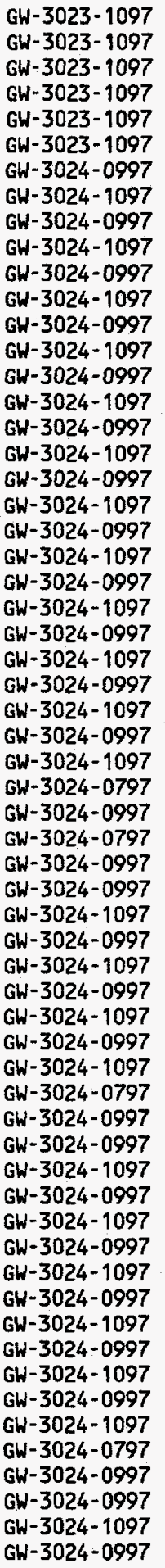 & $\begin{array}{l}10 / 06 / 97 \\
10 / 06 / 97 \\
10 / 06 / 97 \\
10 / 06 / 97 \\
10 / 06 / 97 \\
10 / 06 / 97 \\
09 / 11 / 97 \\
10 / 13 / 97 \\
09 / 11 / 97 \\
10 / 13 / 97 \\
09 / 11 / 97 \\
10 / 13 / 97 \\
09 / 11 / 97 \\
10 / 13 / 97 \\
09 / 11 / 97 \\
10 / 13 / 97 \\
09 / 11 / 97 \\
10 / 13 / 97 \\
09 / 11 / 97 \\
10 / 13 / 97 \\
09 / 11 / 97 \\
10 / 13 / 97 \\
09 / 11 / 97 \\
10 / 13 / 97 \\
09 / 11 / 97 \\
10 / 13 / 97 \\
09 / 11 / 97 \\
10 / 13 / 97 \\
09 / 11 / 97 \\
10 / 13 / 97 \\
07 / 11 / 97 \\
09 / 11 / 97 \\
07 / 11 / 97 \\
09 / 11 / 97 \\
09 / 11 / 97 \\
10 / 13 / 97 \\
09 / 11 / 97 \\
10 / 13 / 97 \\
09 / 11 / 97 \\
10 / 13 / 97 \\
09 / 111 / 97 \\
10 / 13 / 97 \\
07 / 11 / 97 \\
09 / 11 / 97 \\
09 / 11 / 97 \\
10 / 13 / 97 \\
09 / 11 / 97 \\
10 / 13 / 97 \\
09 / 11 / 97 \\
10 / 13 / 97 \\
09 / 11 / 97 \\
10 / 13 / 97 \\
09 / 11 / 97 \\
10 / 13 / 97 \\
09 / 111 / 97 \\
10 / 13 / 97 \\
07 / 11 / 97 \\
09 / 11 / 97 \\
09 / 11 / 97 \\
10 / 13 / 97 \\
09 / 111 / 97\end{array}$ & 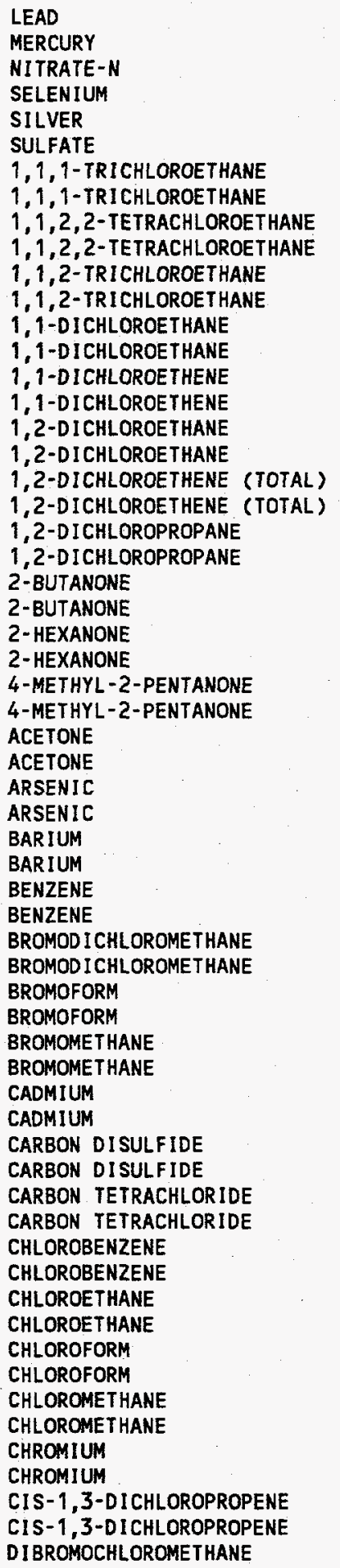 & $\begin{array}{l}\text { ND } \\
1.2 \\
160 \\
5.8 \\
\text { ND } \\
169 \\
\text { ND } \\
\text { ND } \\
\text { ND } \\
\text { ND } \\
\text { ND } \\
\text { ND } \\
\text { ND } \\
\text { ND } \\
\text { ND } \\
\text { ND } \\
\text { ND } \\
\text { ND } \\
\text { ND } \\
\text { ND } \\
\text { ND } \\
\text { ND } \\
\text { ND } \\
\text { ND } \\
\text { ND } \\
\text { ND } \\
\text { ND } \\
\text { ND } \\
\text { ND } \\
\text { ND } \\
\text { ND } \\
\text { ND } \\
\text { C74 } \\
\text { ND } \\
\text { ND } \\
\text { ND } \\
\text { ND } \\
\text { ND } \\
\text { ND } \\
\text { ND } \\
\text { ND } \\
\text { ND } \\
\text { ND } \\
\text { ND } \\
\text { ND } \\
\text { ND } \\
\text { ND } \\
\text { ND } \\
\text { ND } \\
\text { ND } \\
\text { ND } \\
\text { ND } \\
\text { ND } \\
\text { ND } \\
\text { ND } \\
\text { ND } \\
\text { ND } \\
\text { ND } \\
\text { ND } \\
\text { ND } \\
\text { ND } \\
\text { ND } \\
\text { ND } \\
\text { ND } \\
\text { ND }\end{array}$ & $\begin{array}{l}2.0 \\
0.10 \\
4 \\
4 \\
5 \\
1.0 \\
10 \\
10 \\
10 \\
10 \\
10 \\
10 \\
10 \\
10 \\
10 \\
10 \\
10 \\
10 \\
10 \\
10 \\
10 \\
10 \\
10 \\
10 \\
10 \\
10 \\
10 \\
10 \\
10 \\
10 \\
10 \\
10 \\
10 \\
10 \\
10 \\
10 \\
10 \\
10 \\
10 \\
1.7 \\
4\end{array}$ & 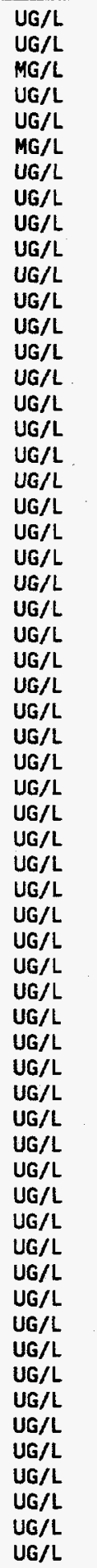 & $\begin{array}{l}\text { EPA } 6010 A \\
\text { EPA } 7470 A \\
\text { EPA } 353.1 \\
\text { EPA } 6010 A \\
\text { EPA } 6010 A \\
\text { EPA } 300.0 \\
\text { EPA CLP } \\
\text { EPA CLP } \\
\text { EPA CLP } \\
\text { EPA CLP } \\
\text { EPA CLP } \\
\text { EPA CLP } \\
\text { EPA CLP } \\
\text { EPA CLP } \\
\text { EPA CLP } \\
\text { EPA CLP } \\
\text { EPA CLP } \\
\text { EPA CLP } \\
\text { EPA CLP } \\
\text { EPA CLP } \\
\text { EPA CLP } \\
\text { EPA CLP } \\
\text { EPA CLP } \\
\text { EPA CLP } \\
\text { EPA CLP } \\
\text { EPA CLP } \\
\text { EPA CLP } \\
\text { EPA CLP } \\
\text { EPA CLP } \\
\text { EPA CLP } \\
\text { EPA CLP } \\
\text { EPA CLP } \\
\text { EPA CLP } \\
\text { EPA CLP } \\
\text { EPA CLP } \\
\text { EPA CLP } \\
\text { EPA CLP } \\
\text { EPA CLP } \\
\text { EPA CLP } \\
\text { EPA CLP } \\
\text { EPA CLP } \\
\text { EPA CLP } \\
\text { EPA CLP } \\
\text { EPA CLP } \\
\text { EPA CLP } \\
\text { EPA CLP } \\
\text { EPA CLP } \\
\text { EPA CLP } \\
\text { EPA CLP } \\
\text { EPA CLP } \\
\text { EPA CLP } \\
\text { EPA CLP } \\
\text { EPA CLP } \\
\text { EPA CLP } \\
\text { EPA CLP } \\
\text { EPA CLP } \\
\text { EPA CLP } \\
\text { EPA CLP } \\
\text { EPA CLP } \\
\text { EPA CLP } \\
\text { EPA CLP }\end{array}$ \\
\hline
\end{tabular}




\begin{tabular}{|c|c|c|c|c|c|c|}
\hline WSSRAP_ID & DATE_SAM & PARAMETER & CONC & $\mathrm{DL}$ & UNITS & METHOD \\
\hline 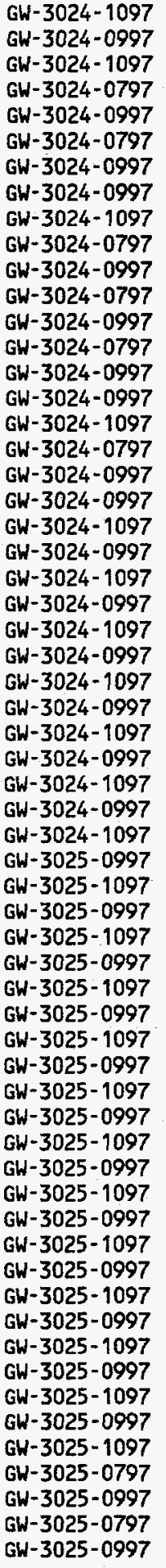 & $\begin{array}{l}10 / 13 / 97 \\
09 / 11 / 97 \\
10 / 13 / 97 \\
07 / 11 / 97 \\
09 / 11 / 97 \\
07 / 11 / 97 \\
09 / 11 / 97 \\
09 / 11 / 97 \\
10 / 13 / 97 \\
07 / 11 / 97 \\
09 / 11 / 97 \\
07 / 11 / 97 \\
09 / 11 / 97 \\
07 / 11 / 97 \\
09 / 11 / 97 \\
09 / 11 / 97 \\
10 / 13 / 97 \\
07 / 11 / 97 \\
09 / 11 / 97 \\
09 / 11 / 97 \\
10 / 13 / 97 \\
09 / 11 / 97 \\
10 / 13 / 97 \\
09 / 11 / 97 \\
10 / 13 / 97 \\
09 / 11 / 97 \\
10 / 13 / 97 \\
09 / 11 / 97 \\
10 / 13 / 97 \\
09 / 11 / 97 \\
10 / 13 / 97 \\
09 / 11 / 97 \\
10 / 13 / 97 \\
09 / 11 / 97 \\
10 / 13 / 97 \\
09 / 11 / 97 \\
10 / 13 / 97 \\
09 / 11 / 97 \\
10 / 13 / 97 \\
09 / 11 / 97 \\
10 / 13 / 97 \\
09 / 11 / 97 \\
10 / 13 / 97 \\
09 / 11 / 97 \\
10 / 13 / 97 \\
09 / 11 / 97 \\
10 / 13 / 97 \\
09 / 11 / 97 \\
10 / 13 / 97 \\
09 / 11 / 97 \\
10 / 13 / 97 \\
09 / 11 / 97 \\
10 / 13 / 97 \\
09 / 11 / 97 \\
10 / 13 / 97 \\
09 / 11 / 97 \\
10 / 13 / 97 \\
07 / 11 / 97 \\
09 / 11 / 97 \\
07 / 11 / 97 \\
09 / 11 / 97\end{array}$ & 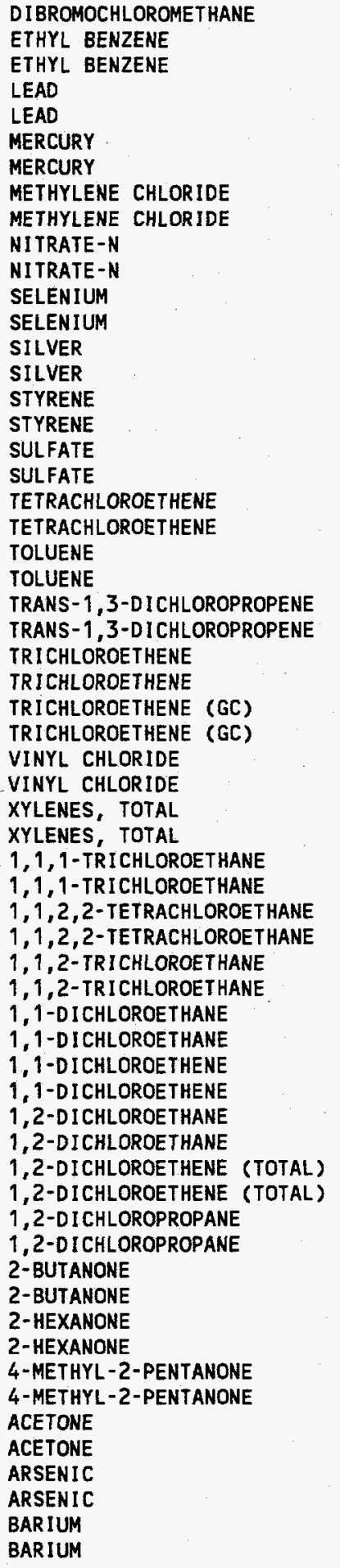 & 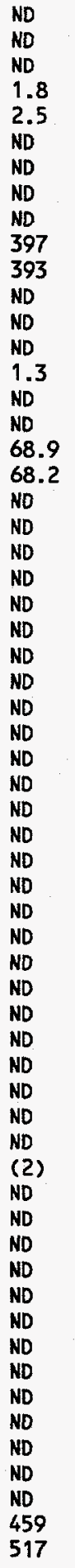 & $\begin{array}{l}10 \\
10 \\
10 \\
10 \\
1.8 \\
2.0 \\
0.10 \\
0.10 \\
10 \\
10 \\
10 \\
\text { S0 } \\
10 \\
10 \\
3\end{array}$ & 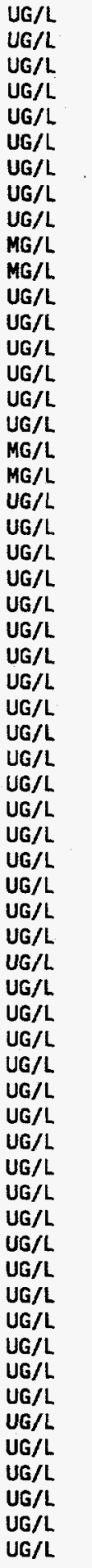 & $\begin{array}{l}\text { EPA CLP } \\
\text { EPA CLP } \\
\text { EPA CLP } \\
\text { EPA CLP } \\
\text { EPA CLP } \\
\text { EPA CLP } \\
\text { EPA CLP } \\
\text { EPA CLP } \\
\text { EPA CLP } \\
\text { EPA } 353.1 \\
\text { EPA } 353.1 \\
\text { EPA CLP } \\
\text { EPA CLP } \\
\text { EPA CLP } \\
\text { EPA CLP } \\
\text { EPA CLP } \\
\text { EPA CLP } \\
\text { EPA } 300.0 \\
\text { EPA } 300.0 \\
\text { EPA CLP } \\
\text { EPA CLP } \\
\text { EPA CLP } \\
\text { EPA CLP } \\
\text { EPA CLP } \\
\text { EPA CLP } \\
\text { EPA CLP } \\
\text { EPA CLP } \\
\text { EPA } 601 \\
\text { EPA } 601 \\
\text { EPA CLP } \\
\text { EPA CLP } \\
\text { EPA CLP } \\
\text { EPA CLP } \\
\text { EPA CLP } \\
\text { EPA CLP } \\
\text { EPA CLP } \\
\text { EPA CLP } \\
\text { EPA CLP } \\
\text { EPA CLP } \\
\text { EPA CLP } \\
\text { EPA CLP } \\
\text { EPA CLP } \\
\text { EPA CLP } \\
\text { EPA CLP } \\
\text { EPA CLP } \\
\text { EPA CLP } \\
\text { EPA CLP } \\
\text { EPA CLP } \\
\text { EPA CLP } \\
\text { EPA CLP } \\
\text { EPA CLP } \\
\text { EPA CLP } \\
\text { EPA CLP } \\
\text { EPA CLP } \\
\text { EPA CLP } \\
\text { EPA CLP } \\
\text { EPA CLP } \\
\text { EPA CLP } \\
\text { EPA CLP } \\
\text { EPA CLP } \\
\text { EPA CLP }\end{array}$ \\
\hline
\end{tabular}




\begin{tabular}{|c|c|c|c|c|c|c|c|}
\hline WSSRAP_ID & DATE_SAM & PARAMETER & CONC & DL & UNITS & METHOD & \\
\hline 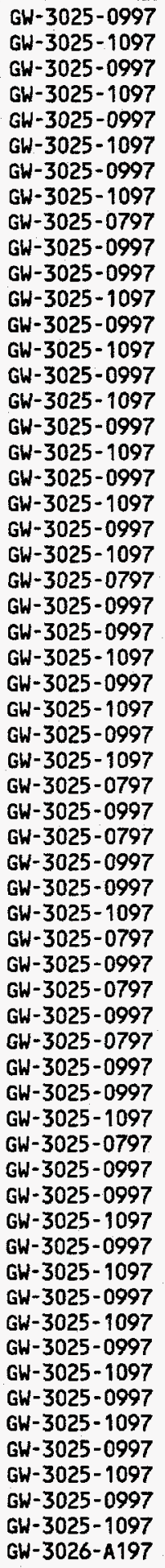 & $\begin{array}{l}09 / 11 / 97 \\
10 / 13 / 97 \\
09 / 11 / 97 \\
10 / 13 / 97 \\
09 / 11 / 97 \\
10 / 13 / 97 \\
09 / 11 / 97 \\
10 / 13 / 97 \\
07 / 11 / 97 \\
09 / 11 / 97 \\
09 / 11 / 97 \\
10 / 13 / 97 \\
09 / 11 / 97 \\
10 / 13 / 97 \\
09 / 11 / 97 \\
10 / 13 / 97 \\
09 / 11 / 97 \\
10 / 13 / 97 \\
09 / 11 / 97 \\
10 / 13 / 97 \\
09 / 11 / 97 \\
10 / 13 / 97 \\
07 / 11 / 97 \\
09 / 11 / 97 \\
09 / 11 / 97 \\
10 / 13 / 97 \\
09 / 111 / 97 \\
10 / 13 / 97 \\
09 / 11 / 97 \\
10 / 13 / 97 \\
07 / 111 / 97 \\
09 / 111 / 97 \\
07 / 11 / 97 \\
09 / 11 / 97 \\
09 / 11 / 97 \\
10 / 13 / 97 \\
07 / 11 / 97 \\
09 / 11 / 97 \\
07 / 11 / 97 \\
09 / 11 / 97 \\
07 / 111 / 97 \\
09 / 11 / 97 \\
09 / 11 / 97 \\
10 / 13 / 97 \\
07 / 111 / 97 \\
09 / 111 / 97 \\
09 / 11 / 97 \\
10 / 13 / 97 \\
09 / 11 / 97 \\
10 / 13 / 97 \\
09 / 111 / 97 \\
10 / 13 / 97 \\
09 / 11 / 97 \\
10 / 13 / 97 \\
09 / 111 / 97 \\
10 / 13 / 97 \\
09 / 11 / 97 \\
10 / 13 / 97 \\
09 / 11 / 97 \\
10 / 13 / 97 \\
08 / 27 / 97\end{array}$ & 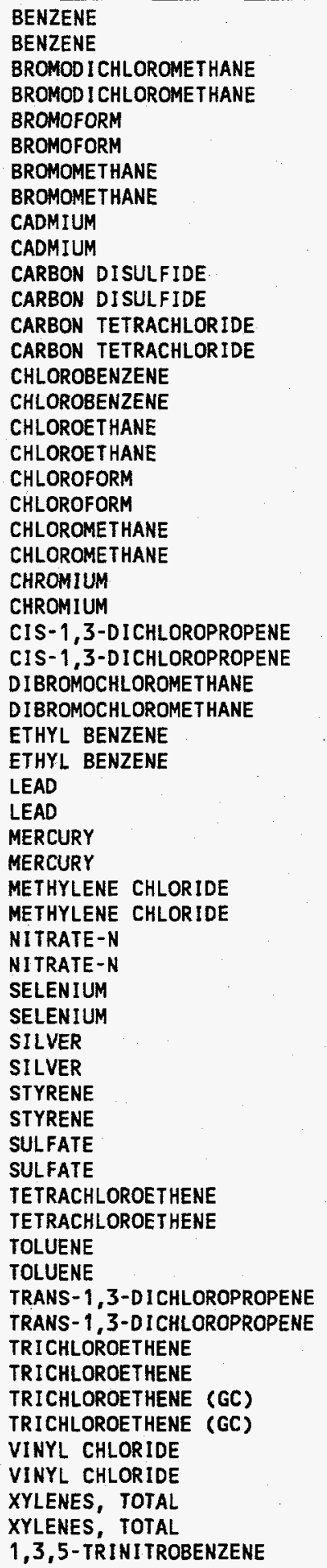 & 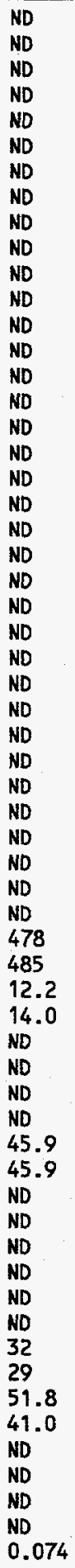 & $\begin{array}{l}10 \\
10 \\
10 \\
10 \\
10 \\
10 \\
10 \\
10 \\
10 \\
2.9 \\
3.0 \\
10 \\
10 \\
10 \\
10 \\
10 \\
10 \\
10 \\
10 \\
10 \\
10 \\
10 \\
10 \\
10 \\
20 \\
2.7 \\
4.0 \\
10 \\
10 \\
10 \\
10 \\
10 \\
10 \\
10 \\
10 \\
1.8 \\
2.0 \\
0.10 \\
0\end{array}$ & 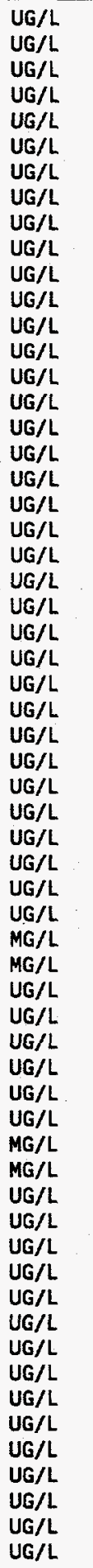 & $\begin{array}{l}\text { EPA CLP } \\
\text { EPA CLP } \\
\text { EPA CLP } \\
\text { EPA CLP } \\
\text { EPA CLP } \\
\text { EPA CLP } \\
\text { EPA CLP } \\
\text { EPA CLP } \\
\text { EPA CLP } \\
\text { EPA CLP } \\
\text { EPA CLP } \\
\text { EPA CLP } \\
\text { EPA CLP } \\
\text { EPA CLP } \\
\text { EPA CLP } \\
\text { EPA CLP } \\
\text { EPA CLP } \\
\text { EPA CLP } \\
\text { EPA CLP } \\
\text { EPA CLP } \\
\text { EPA CLP } \\
\text { EPA CLP } \\
\text { EPA CLP } \\
\text { EPA CLP } \\
\text { EPA CLP } \\
\text { EPA CLP } \\
\text { EPA CLP } \\
\text { EPA CLP } \\
\text { EPA CLP } \\
\text { EPA CLP } \\
\text { EPA CLP } \\
\text { EPA CLP } \\
\text { EPA CLP } \\
\text { EPA CLP } \\
\text { EPA CLP } \\
\text { EPA CLP } \\
\text { EPA } 353.1 \\
\text { EPA } 353.1 \\
\text { EPA CLP } \\
\text { EPA CLP } \\
\text { EPA CLP } \\
\text { EPA CLP } \\
\text { EPA CLP } \\
\text { EPA CLP } \\
\text { EPA } 300.0 \\
\text { EPA } 300.0 \\
\text { EPA CLP } \\
\text { EPA CLP } \\
\text { EPA CLP } \\
\text { EPA CLP } \\
\text { EPA CLP } \\
\text { EPA CLP } \\
\text { EPA CLP } \\
\text { EPA CLP } \\
\text { EPA } 601 \\
\text { EPA } 601 \\
\text { EPA CLP } \\
\text { EPA CLP } \\
\text { EPA CLP } \\
\text { EPA CLP } \\
\text { USATHAMA }\end{array}$ & $*$ \\
\hline
\end{tabular}




\begin{tabular}{|c|c|c|c|c|c|c|}
\hline WSSRAP_ID & DATE_SAM & PARAMETER & CONC & $\mathrm{DL}$ & UNITS & METHOD \\
\hline 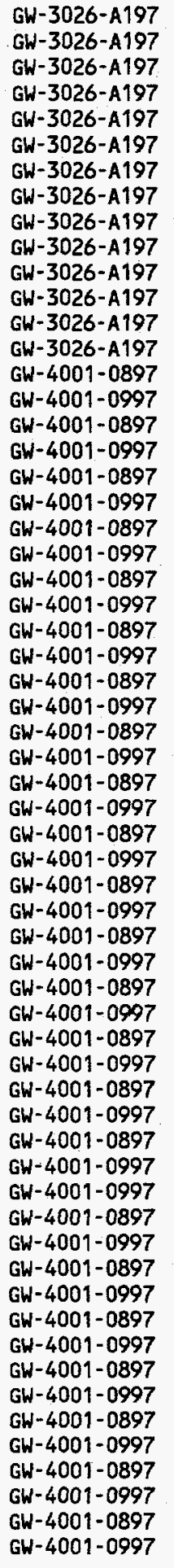 & $\begin{array}{l}08 / 27 / 97 \\
08 / 27 / 97 \\
08 / 27 / 97 \\
08 / 27 / 97 \\
08 / 27 / 97 \\
08 / 27 / 97 \\
08 / 27 / 97 \\
08 / 27 / 97 \\
08 / 27 / 97 \\
08 / 27 / 97 \\
08 / 27 / 97 \\
08 / 27 / 97 \\
08 / 27 / 97 \\
08 / 27 / 97 \\
08 / 18 / 97 \\
09 / 22 / 97 \\
08 / 18 / 97 \\
09 / 22 / 97 \\
08 / 18 / 97 \\
09 / 22 / 97 \\
08 / 18 / 97 \\
09 / 22 / 97 \\
08 / 18 / 97 \\
09 / 22 / 97 \\
08 / 18 / 97 \\
09 / 22 / 97 \\
08 / 18 / 97 \\
09 / 22 / 97 \\
08 / 18 / 97 \\
09 / 22 / 97 \\
08 / 18 / 97 \\
09 / 22 / 97 \\
08 / 18 / 97 \\
09 / 22 / 97 \\
08 / 18 / 97 \\
09 / 22 / 97 \\
08 / 18 / 97 \\
09 / 22 / 97 \\
08 / 18 / 97 \\
09 / 22 / 97 \\
08 / 18 / 97 \\
09 / 22 / 97 \\
08 / 18 / 97 \\
09 / 22 / 97 \\
08 / 18 / 97 \\
09 / 22 / 97 \\
09 / 22 / 97 \\
08 / 18 / 97 \\
09 / 22 / 97 \\
08 / 18 / 97 \\
09 / 22 / 97 \\
08 / 18 / 97 \\
09 / 22 / 97 \\
08 / 18 / 97 \\
09 / 22 / 97 \\
08 / 18 / 97 \\
09 / 22 / 97 \\
08 / 18 / 97 \\
09 / 22 / 97 \\
08 / 18 / 97 \\
09 / 22 / 97\end{array}$ & 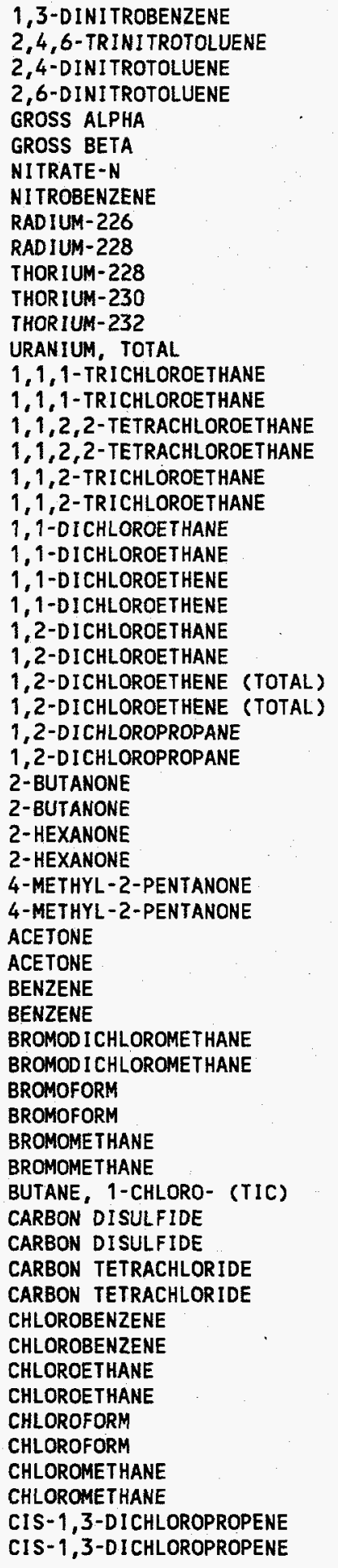 & 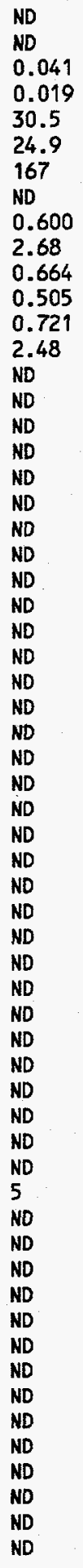 & 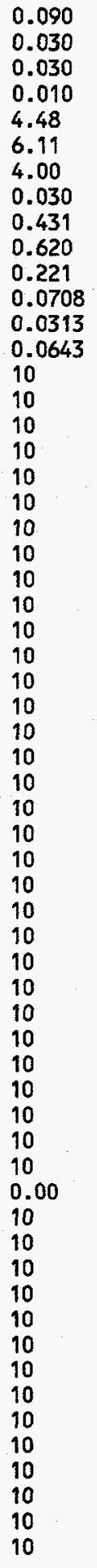 & 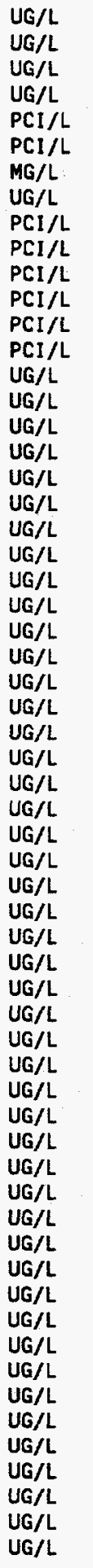 & $\begin{array}{l}\text { USATHAMA } \\
\text { USATHAMA } \\
\text { USATHAMA } \\
\text { USATHAMA } \\
\text { EPA } 900: 0 \\
\text { EPA } 900.0 \\
\text { EPA } 353.1 \\
\text { USATHAMA } \\
\text { EPA 903.1 } \\
\text { EPA 904.0 } \\
\text { HASL } 300 \\
\text { HASL } 300 \\
\text { HASL } 300 \\
\text { ASTM } 5174-91 \\
\text { EPA CLP } \\
\text { EPA CLP } \\
\text { EPA CLP } \\
\text { EPA CLP } \\
\text { EPA CLP } \\
\text { EPA CLP } \\
\text { EPA CLP } \\
\text { EPA CLP } \\
\text { EPA CLP } \\
\text { EPA CLP } \\
\text { EPA CLP } \\
\text { EPA CLP } \\
\text { EPA CLP } \\
\text { EPA CLP } \\
\text { EPA CLP } \\
\text { EPA CLP } \\
\text { EPA CLP } \\
\text { EPA CLP } \\
\text { EPA CLP } \\
\text { EPA CLP } \\
\text { EPA CLP } \\
\text { EPA CLP } \\
\text { EPA CLP } \\
\text { EPA CLP } \\
\text { EPA CLP } \\
\text { EPA CLP } \\
\text { EPA CLP } \\
\text { EPA CLP } \\
\text { EPA CLP } \\
\text { EPA CLP } \\
\text { EPA CLP } \\
\text { EPA CLP } \\
\text { EPA CLP } \\
\text { EPA CLP } \\
\text { EPA CLP } \\
\text { EPA CLP } \\
\text { EPA CLP } \\
\text { EPA CLP } \\
\text { EPA CLP } \\
\text { EPA CLP } \\
\text { EPA CLP } \\
\text { EPA CLP } \\
\text { EPA CLP } \\
\text { EPA CLP } \\
\text { EPA CLP } \\
\text { EPA CLP } \\
\text { EPA CLP } \\
\end{array}$ \\
\hline
\end{tabular}




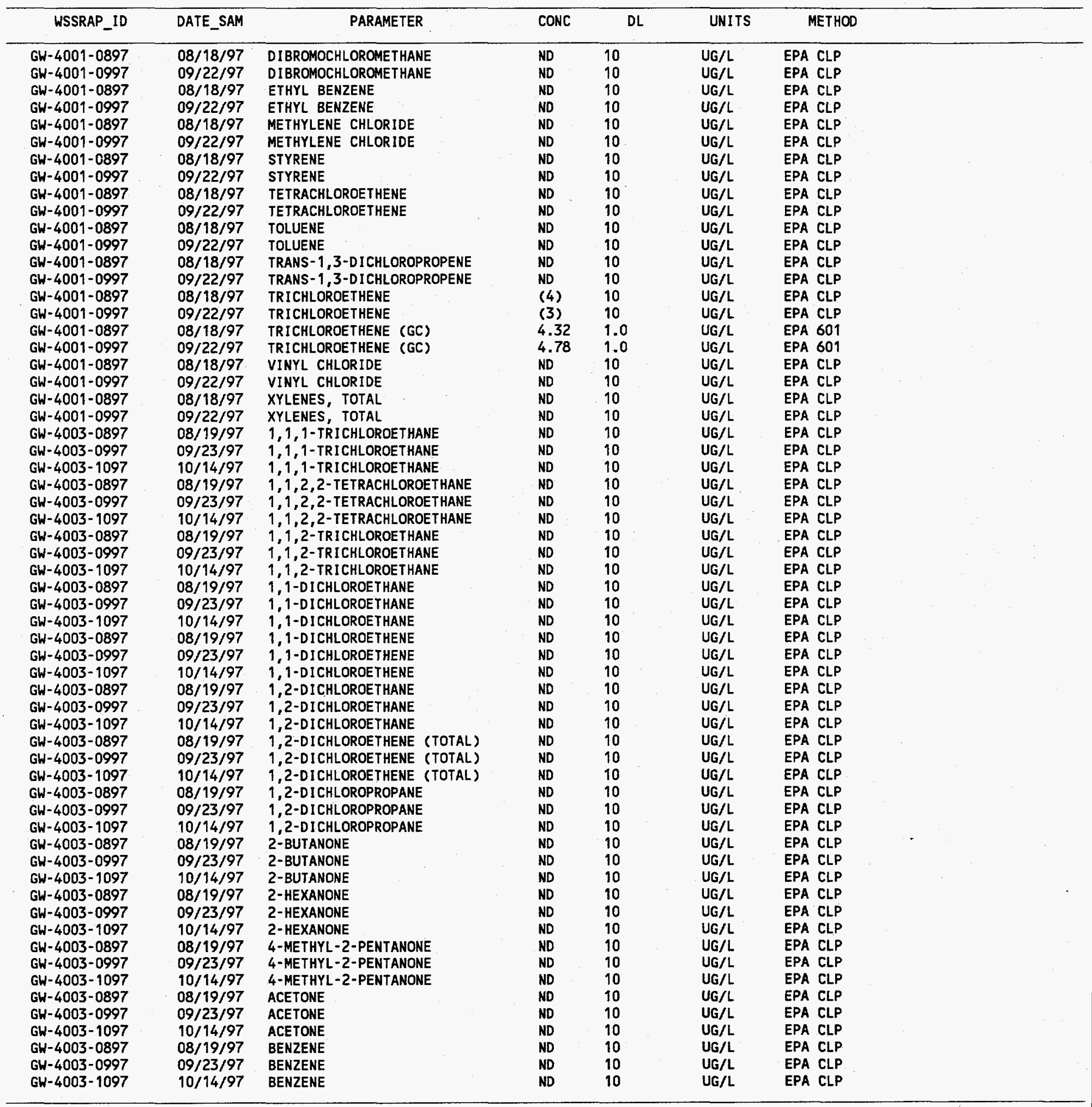




\begin{tabular}{|c|c|c|c|c|c|c|c|}
\hline WSSRAP_ID & DATE_SAM & PARAMETER & CONC & $D L$ & UNITS & METHOD & \\
\hline $\begin{array}{l}\text { GW-4003-0897 } \\
\text { GW-4003-0997 } \\
\text { GW-4003-1097 } \\
\text { GW-4003-0897 } \\
\text { GW-4003-0997 } \\
\text { GW-4003-1097 } \\
\text { GW-4003-0897 } \\
\text { GW-4003-0997 } \\
\text { GW-4003-1097 } \\
\text { GW-4003-0997 } \\
\text { GW-4003-0897 } \\
\text { GW-4003-0997 } \\
\text { GW-4003-1097 } \\
\text { GW-4003-0897 } \\
\text { GW-4003-0997 } \\
\text { GW-4003-1097 } \\
\text { GW-4003-0897 } \\
\text { GW-4003-0997 } \\
\text { GW-4003-1097 } \\
\text { GW-4003-0897 } \\
\text { GW-4003-0997 } \\
\text { GW-4003-1097 } \\
\text { GW-4003-0897 } \\
\text { GW-4003-0997 } \\
\text { GW-4003-1097 } \\
\text { GW-4003-0897 } \\
\text { GW-4003-0997 } \\
\text { GW-4003-1097 } \\
\text { GW-4003-0897 } \\
\text { GW-4003-0997 } \\
\text { GW-4003-1097 } \\
\text { GW-4003-0897 } \\
\text { GW-4003-0997 } \\
\text { GW-4003-1097 } \\
\text { GW-4003-0897 } \\
\text { GW-4003-0997 } \\
\text { GW-4003-1097 } \\
\text { GW-4003-0897 } \\
\text { GW-4003-0997 } \\
\text { GW-4003-1097 } \\
\text { GW-4003-0897 } \\
\text { GW-4003-0997 } \\
\text { GW-4003-1097 } \\
\text { GW-4W-4003-0897 } \\
\text { GW-4003-0997 } \\
\text { GW-4003-1097 } \\
\text { GW-4003-0897 } \\
\text { GW-4003-0997 } \\
\text { GW-4003-1097 } \\
\text { GW-4003-4003-003-0997 } \\
\text { GW-4003-4003-0997 } \\
\text { GW-4003-1097 } \\
\text { GW-4003-0897 } \\
\text { GW-4003-0997 }\end{array}$ & $\begin{array}{l}08 / 19 / 97 \\
09 / 23 / 97 \\
10 / 14 / 97 \\
08 / 19 / 97 \\
09 / 23 / 97 \\
10 / 14 / 97 \\
08 / 19 / 97 \\
09 / 23 / 97 \\
10 / 14 / 97 \\
09 / 23 / 97 \\
08 / 19 / 97 \\
09 / 23 / 97 \\
10 / 14 / 97 \\
08 / 19 / 97 \\
09 / 23 / 97 \\
10 / 14 / 97 \\
08 / 19 / 97 \\
09 / 23 / 97 \\
10 / 14 / 97 \\
08 / 19 / 97 \\
09 / 23 / 97 \\
10 / 14 / 97 \\
08 / 19 / 97 \\
09 / 23 / 97 \\
10 / 14 / 97 \\
08 / 19 / 97 \\
09 / 23 / 97 \\
10 / 14 / 97 \\
08 / 19 / 97 \\
09 / 23 / 97 \\
10 / 14 / 97 \\
08 / 19 / 97 \\
09 / 23 / 97 \\
10 / 14 / 97 \\
08 / 19 / 97 \\
09 / 23 / 97 \\
10 / 14 / 97 \\
08 / 19 / 97 \\
09 / 23 / 97 \\
10 / 14 / 97 \\
08 / 19 / 97 \\
09 / 23 / 97 \\
10 / 14 / 97 \\
08 / 19 / 97 \\
09 / 23 / 97 \\
10 / 14 / 97 \\
08 / 19 / 97 \\
09 / 23 / 97 \\
10 / 14 / 97 \\
08 / 19 / 97 \\
09 / 23 / 97 \\
10 / 14 / 97 \\
08 / 19 / 97 \\
09 / 23 / 97 \\
10 / 14 / 97 \\
08 / 19 / 97 \\
09 / 23 / 97 \\
10 / 14 / 97 \\
08 / 19 / 97 \\
09 / 23 / 97 \\
10 / 14 / 97\end{array}$ & 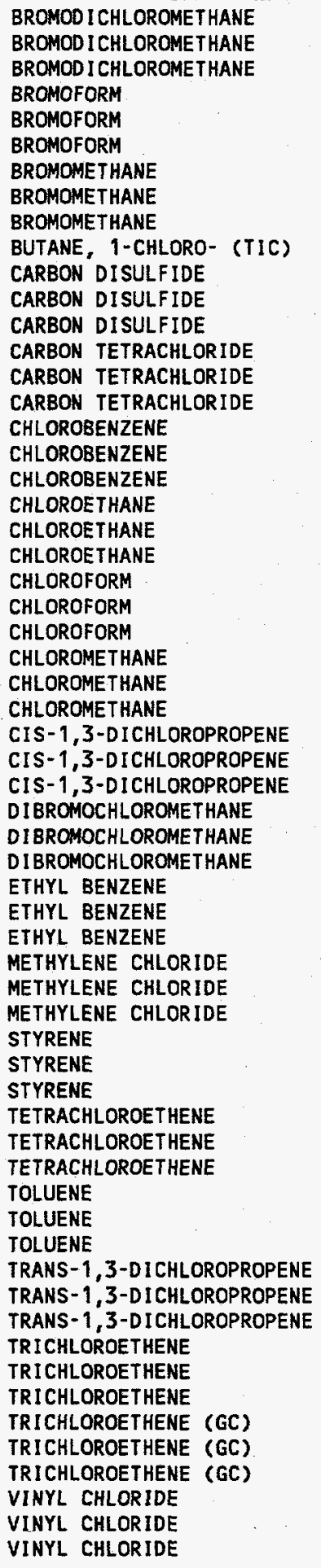 & $\begin{array}{l}\text { ND } \\
\text { ND } \\
\text { ND } \\
\text { ND } \\
\text { ND } \\
\text { ND } \\
\text { ND } \\
\text { ND } \\
\text { ND } \\
5 \\
\text { ND } \\
\text { ND } \\
\text { ND } \\
\text { ND } \\
\text { ND } \\
\text { ND } \\
\text { ND } \\
\text { ND } \\
\text { ND } \\
\text { ND } \\
\text { ND } \\
\text { ND } \\
\text { ND } \\
\text { ND } \\
\text { ND } \\
\text { ND } \\
\text { ND } \\
\text { ND } \\
\text { ND } \\
\text { ND } \\
\text { ND } \\
\text { ND } \\
\text { ND } \\
\text { ND } \\
\text { ND } \\
\text { ND } \\
\text { ND } \\
\text { ND } \\
\text { ND } \\
\text { ND } \\
\text { ND } \\
\text { ND } \\
\text { ND } \\
\text { ND } \\
\text { ND } \\
\text { ND } \\
\text { ND } \\
\text { ND } \\
\text { ND } \\
\text { ND } \\
\text { ND } \\
\text { ND } \\
\text { ND } \\
\text { ND } \\
\text { ND } \\
\text { ND } \\
\text { ND } \\
\text { ND } \\
\text { ND } \\
\text { ND } \\
\text { ND }\end{array}$ & $\begin{array}{l}10 \\
10 \\
10 \\
10 \\
10 \\
10 \\
10 \\
10 \\
10 \\
10 \\
10 \\
0 \\
0 \\
10 \\
10 \\
10 \\
10 \\
10 \\
10 \\
10 \\
10 \\
10 \\
10 \\
10 \\
10 \\
10 \\
10 \\
10 \\
10 \\
10 \\
10 \\
10 \\
10\end{array}$ & 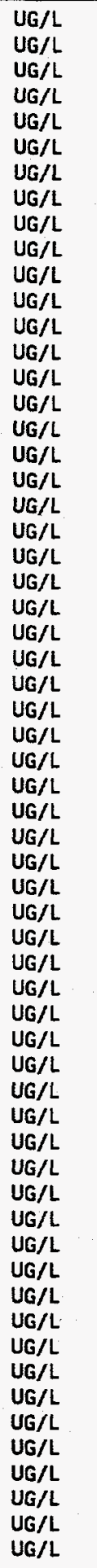 & 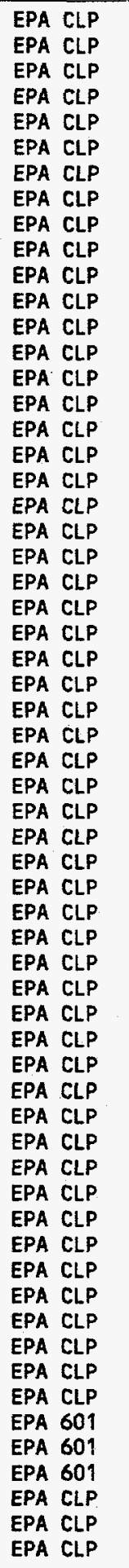 & . \\
\hline
\end{tabular}




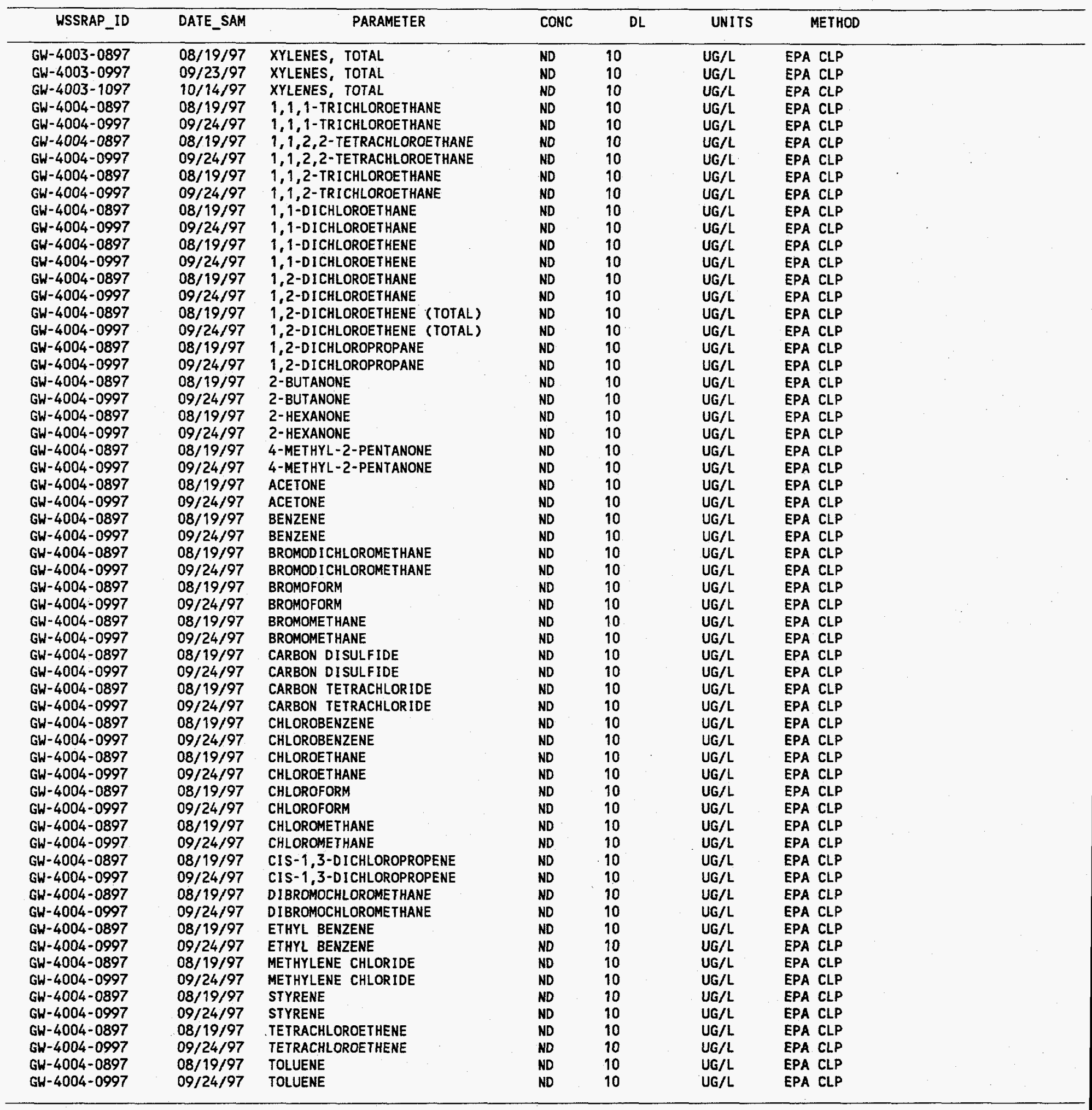




\begin{tabular}{|c|c|c|c|c|c|c|}
\hline WSSRAP_1D & DATE_SAM & PARAMETER & CONC & DL & UNITS & METHOD \\
\hline $\begin{array}{l}\text { GW-4004-0897 } \\
\text { GW-4004-0997 } \\
\text { GW-4004-0897 } \\
\text { GW-4004-0997 } \\
\text { GW-4004-0897 } \\
\text { GW-4004-0997 } \\
\text { GW-4004-0897 } \\
\text { GW-4004-0997 } \\
\text { GW-4004-0897 } \\
\text { GW-4004-0997 } \\
\text { GW-4005-0997 } \\
\text { GW-4005-0997 } \\
\text { GW-4005-0997 } \\
\text { GW-4005-0997 } \\
\text { GW-4005-0997 } \\
\text { GW-4005-0997 } \\
\text { GW-4005-0997 } \\
\text { GW-4005-0997 } \\
\text { GW-4005-0997 } \\
\text { GW-4005-0997 } \\
\text { GW-4005-0997 } \\
\text { GW-4005-0997 } \\
\text { GW-4005-0997 } \\
\text { GW-4005-0997 } \\
\text { GW-4005-0997 } \\
\text { GW-4005-0997 } \\
\text { GW-4005-0997 } \\
\text { GW-4005-0997 } \\
\text { GW-4005-0997 } \\
\text { GW-4005-0997 } \\
\text { GW-4005-0997 } \\
\text { GW-4005-0997 } \\
\text { GW-4005-0997 } \\
\text { GW-4005-0997 } \\
\text { GW-4005-0997 } \\
\text { GW-4005-09977 } \\
\text { GW-4005-0997 } \\
\text { GW-4005-0997 } \\
\text { GW-4005-0997 } \\
\text { GW-4005-0997 } \\
\text { GW-4005-0997 } \\
\text { GW-4005-0997- }\end{array}$ & $\begin{array}{l}08 / 19 / 97 \\
09 / 24 / 97 \\
08 / 19 / 97 \\
09 / 24 / 97 \\
08 / 19 / 97 \\
09 / 24 / 97 \\
08 / 19 / 97 \\
09 / 24 / 97 \\
08 / 19 / 97 \\
09 / 24 / 97 \\
09 / 24 / 97 \\
09 / 24 / 97 \\
09 / 24 / 97 \\
09 / 24 / 97 \\
09 / 24 / 97 \\
09 / 24 / 97 \\
09 / 24 / 97 \\
09 / 24 / 97 \\
09 / 24 / 97 \\
09 / 24 / 97 \\
09 / 24 / 97 \\
09 / 24 / 97 \\
09 / 24 / 97 \\
09 / 24 / 97 \\
09 / 24 / 97 \\
09 / 24 / 97 \\
09 / 24 / 97 \\
09 / 24 / 97 \\
09 / 24 / 97 \\
09 / 24 / 97 \\
09 / 24 / 97 \\
09 / 24 / 97 \\
09 / 24 / 97 \\
09 / 24 / 97 \\
09 / 24 / 97 \\
09 / 24 / 97 \\
09 / 24 / 97 \\
09 / 24 / 97 \\
09 / 24 / 97 \\
09 / 24 / 97 \\
09 / 24 / 97 \\
09 / 24 / 97 \\
09 / 24 / 97 \\
09 / 24 / 97 \\
09 / 23 / 97 \\
09 / 23 / 97 \\
09 / 23 / 97 \\
09 / 23 / 97 \\
09 / 23 / 97 \\
09 / 23 / 97 \\
09 / 23 / 97 \\
09 / 23 / 97 \\
09 / 23 / 97 \\
09 / 23 / 97 \\
09 / 23 / 97 \\
09 / 23 / 97 \\
09 / 23 / 97 \\
09 / 23 / 97 \\
09 / 23 / 97 \\
09 / 23 / 97 \\
09 / 23 / 97\end{array}$ & 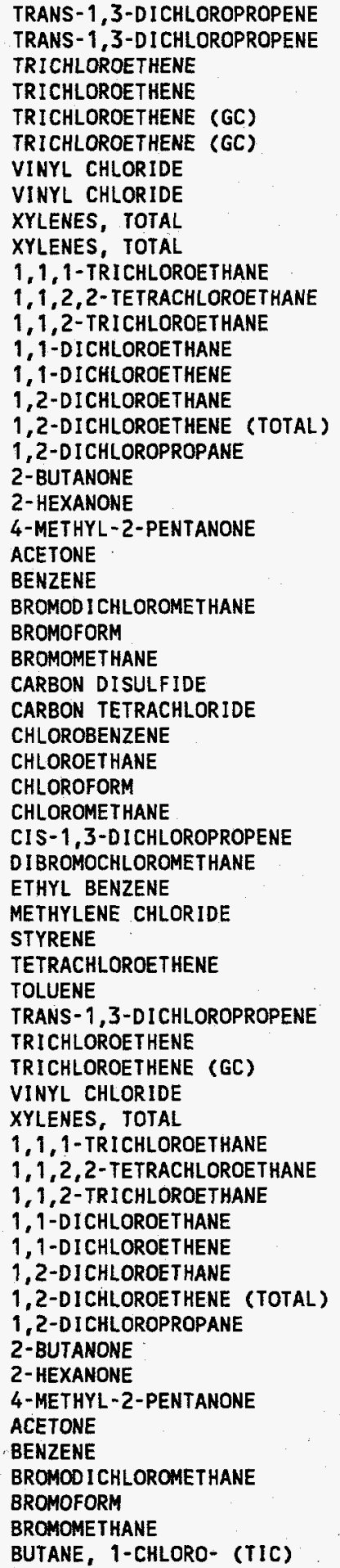 & $\begin{array}{l}\text { ND } \\
\text { ND } \\
\text { ND } \\
\text { ND } \\
\text { ND } \\
\text { ND } \\
\text { ND } \\
\text { ND } \\
\text { ND } \\
\text { ND } \\
\text { ND } \\
\text { ND } \\
\text { ND } \\
\text { ND } \\
\text { ND } \\
\text { ND } \\
\text { ND } \\
\text { ND } \\
\text { ND } \\
\text { ND } \\
\text { ND } \\
\text { ND } \\
\text { ND } \\
\text { ND } \\
\text { ND } \\
\text { ND } \\
\text { ND } \\
\text { ND } \\
\text { ND } \\
\text { ND } \\
\text { ND } \\
\text { ND } \\
\text { ND } \\
\text { ND } \\
\text { ND } \\
\text { ND } \\
\text { ND } \\
\text { ND } \\
\text { ND } \\
\text { ND } \\
\text { ND } \\
\text { ND } \\
\text { ND } \\
\text { ND } \\
\text { ND } \\
\text { ND } \\
\text { ND } \\
\text { ND } \\
\text { ND } \\
\text { ND } \\
\text { ND } \\
\text { ND } \\
\text { ND } \\
\text { ND } \\
\text { ND } \\
\text { ND } \\
\text { ND } \\
\text { ND } \\
\text { ND } \\
\text { ND } \\
3\end{array}$ & $\begin{array}{l}10 \\
10 \\
10 \\
10 \\
10 \\
10 \\
10 \\
10 \\
10 \\
10 \\
10 \\
10 \\
10 \\
10 \\
10 \\
10 \\
10 \\
10 \\
10 \\
10 \\
10 \\
10 \\
10 \\
10 \\
10 \\
10 \\
10 \\
10 \\
10 \\
10\end{array}$ & 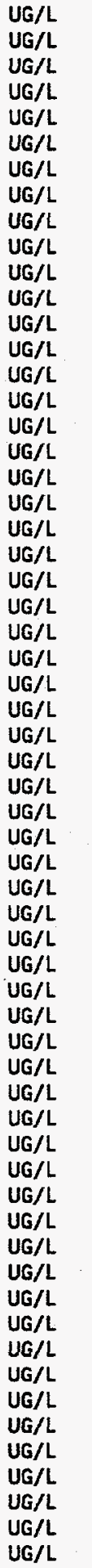 & 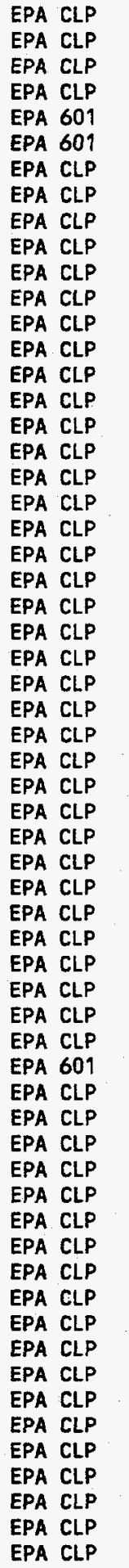 \\
\hline
\end{tabular}




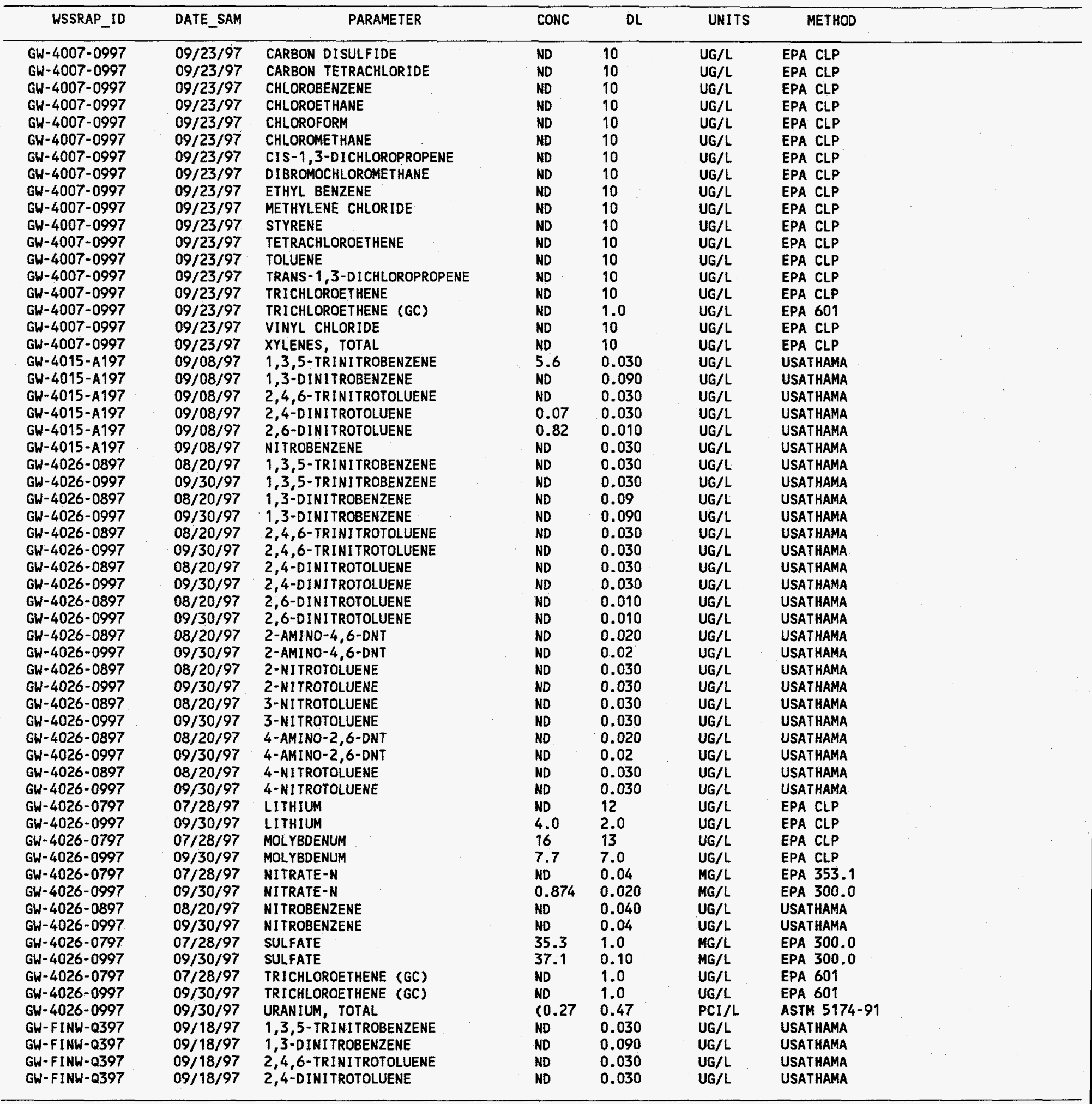




\begin{tabular}{|c|c|c|c|c|c|c|}
\hline WSSRAP_ID & DATE_SAM & PARAMETER & CONC & $\mathrm{DL}$ & UNITS & METHOD \\
\hline GW-FINW-Q397 & $09 / 18 / 97$ & 2,6 -DINITROTOLUENE & ND & 0.010 & $U G / L$ & USATHAMA \\
\hline GW-FINW-Q397 & $09 / 18 / 97$ & NITROBENZENE & ND & 0.030 & $U G / L$ & USATHAMA \\
\hline GW-PW02-0397 & $09 / 18 / 97$ & 1,3,5-TRINITROBENZENE & ND & 0.030 & UG/L & USATHAMA \\
\hline GW-PW02-Q397 & $09 / 18 / 97$ & 1,3-DINI TROBENZENE & ND & 0.090 & UG/L & USATHAMA \\
\hline GW-PW02-Q397 & $09 / 18 / 97$ & 2,4,6-TRINITROTOLUENE & ND & 0.030 & $U G / L$ & USATHAMA \\
\hline GW-PW02-0397 & $09 / 18 / 97$ & 2,4-DINITROTOLUENE & ND & 0.030 & UG/L & USATHAMA \\
\hline GW-PW02-0397 & $09 / 18 / 97$ & 2,6-DINITROTOLUENE & ND & 0.010 & UG/L & USATHAMA \\
\hline GW-PW02-0397 & $09 / 18 / 97$ & NITROBENZENE & ND & 0.030 & UG $/ L$ & USATHAMA \\
\hline GW-PW03-0397 & $09 / 18 / 97$ & 1,3,5-TR IN I TROBENZENE & ND & 0.030 & $U G / L$ & USATHAMA \\
\hline GW-PW03-Q397 & $09 / 18 / 97$ & 1,3-DINITROBENZENE & ND & 0.090 & UG/L & USATHAMA \\
\hline GW-PW03-0397 & $09 / 18 / 97$ & $2,4,6$-TRINITROTOLUENE & ND & 0.030 & UG/L & USATHAMA \\
\hline GW-PW03-Q397 & $09 / 18 / 97$ & 2,4-DINITROTOLUENE & ND & 0.030 & $U G / L$ & USATHAMA \\
\hline GW-PW03-0397 & $09 / 18 / 97$ & 2,6-DINITROTOLUENE & ND & 0.010 & UG/L & USATHAMA \\
\hline GW-PW03-0397 & $09 / 18 / 97$ & NI TROBENZENE & ND & 0.030 & UG/L & USATHAMA \\
\hline GW-PW04-Q397 & $09 / 18 / 97$ & $1,3,5$-TRINITROBENZENE & ND & 0.030 & UG $/ L$ & USATHAMA \\
\hline GW-PWO4-0397 & $09 / 18 / 97$ & 1,3-DINI TROBENZENE & ND & 0.090 & UG/L & USATHAMA \\
\hline GW-PW04-0397 & $09 / 18 / 97$ & 2,4,6-TRINITROTOLUENE & ND & 0.030 & UG/L & USATHAMA \\
\hline GW-PW04-0397 & $09 / 18 / 97$ & 2,4-DINITROTOLUENE & ND & 0.030 & $U G / L$ & USATHAMA \\
\hline GW-PW04-0397 & $09 / 18 / 97$ & 2,6-DINITROTOLUENE & ND & 0.010 & UG/L & USATHAMA \\
\hline GW-PW04-0397 & $09 / 18 / 97$ & NI TROBENZENE & ND & 0.030 & UG/L & USATHAMA \\
\hline GW-PW05-Q397 & $09 / 18 / 97$ & $1,3,5$-TRINITROBENZENE & ND & 0.030 & UG/L & USATHAMA \\
\hline GW-PW05-0397 & $09 / 18 / 97$ & 1,3 -DINITROBENZENE & ND & 0.090 & UG/L & USATHAMA \\
\hline GW-PW05-0397 & $09 / 18 / 97$ & 2,4,6-TRINITROTOLUENE & ND & 0.030 & UG $/ L$ & USATHAMA \\
\hline GW-PW05-Q397 & $09 / 18 / 97$ & 2,4-D INITROTOLUENE & ND & 0.030 & $U G / L$ & USATHAMA \\
\hline GW-PW05-0397 & $09 / 18 / 97$ & 2,6-DINITROTOLUENE & ND & 0.010 & $U G / L$ & USATHAMA \\
\hline GW-PW05-Q397 & $09 / 18 / 97$ & NITROBENZENE & ND & 0.030 & UG/L & USATHAMA \\
\hline GW-PW06-Q397 & $09 / 18 / 97$ & 1,3,5-TRINITROBENZENE & ND & 0.030 & $U G / L$ & USATHAMA \\
\hline GW-PW06-0397 & $09 / 18 / 97$ & 1,3-DINITROBENZENE & ND & 0.090 & UG/L & USATHAMA \\
\hline GW-PW06-Q397 & $09 / 18 / 97$ & $2,4,6$-TRINITROTOLUENE & ND & 0.030 & UG/L & USATHAMA \\
\hline GW-PW06-0397 & $09 / 18 / 97$ & 2,4-DINITROTOLUENE & ND & 0.030 & $U G / L$ & USATHAMA \\
\hline GW-PW06-0397 & $09 / 18 / 97$ & 2,6-DINITROTOLUENE & ND & 0.010 & UG $/ L$ & USATHAMA \\
\hline GW-PW06-0397 & $09 / 18 / 97$ & NITROBENZENE & ND & 0.030 & UG/L & USATHAMA \\
\hline GW-PW07-Q397 & $09 / 18 / 97$ & $1,3,5$-TRINITROBENZENE & ND & 0.030 & $U G / L$ & USATHAMA \\
\hline GW-PW07-0397 & $09 / 18 / 97$ & 1,3 -DINITROBENZENE & ND & 0.090 & $U G / L$ & USATHAMA \\
\hline GW-PW07-0397 & $09 / 18 / 97$ & 2,4,6-TRINITROTOLUENE & ND & 0.030 & UG/L & USATHAMA \\
\hline GW-PW07-0397 & $09 / 18 / 97$ & 2,4-DINI TROTOLUENE & ND & 0.030 & UG/L & USATHAMA \\
\hline GW-PW07-0397 & $09 / 18 / 97$ & 2,6-DINITROTOLUENE & NO & 0.010 & UG/L & USATHAMA \\
\hline GW-PW07-Q397 & $09 / 18 / 97$ & NITROBENZENE & ND & 0.030 & UG/L & USATHAMA \\
\hline GW-PW08-0397 & $09 / 18 / 97$ & $1,3,5$-TRINITROBENZENE & ND & 0.030 & UG/L & USATHAMA \\
\hline GW-PW08-0397 & $09 / 18 / 97$ & 1,3 -DINI TROBENZENE & ND & 0.090 & $\mathrm{UG} / \mathrm{L}$ & USATHAMA \\
\hline GW-PW08-Q397 & $09 / 18 / 97$ & 2,4,6-TRINITROTOLUENE & ND & 0.030 & $U G / L$ & USATHAMA \\
\hline GW-PWD8-Q397 & $09 / 18 / 97$ & 2,4-DINITROTOLUENE & ND & 0.030 & UG $/ L$ & USATHAMA \\
\hline GW-PW08-Q397 & $09 / 18 / 97$ & 2,6-DINITROTOLUENE & ND & 0.010 & UG/L & USATHAMA \\
\hline GW-PW08-Q397 & $09 / 18 / 97$ & NITROBENZENE & ND & 0.030 & $U G / L$ & USATHAMA \\
\hline GW-PW09-Q397 & $09 / 18 / 97$ & $1,3,5$-TRINITROBENZENE & ND & 0.030 & $U G / L$ & USATHAMA \\
\hline GW-PW09-Q397 & $09 / 18 / 97$ & 1,3-DINITROBENZENE & ND & 0.090 & $U G / L$ & USATHAMA \\
\hline GW-PW09-0397 & $09 / 18 / 97$ & $2,4,6$-TRINITROTOLUENE & ND & 0.030 & UG/L & USATHAMA \\
\hline GW-PW09-0397 & $09 / 18 / 97$ & 2,4-DINITROTOLUENE & ND & 0.030 & $U G / L$ & USATHAMA \\
\hline GW-PW09-Q397 & $09 / 18 / 97$ & 2,6-DINITROTOLUENE & ND & 0.010 & $U G / L$ & USATHAMA \\
\hline GW-PW09-0397 & $09 / 18 / 97$ & NITROBENZENE & ND & 0.030 & $U G / L$ & USATHAMA \\
\hline GW-RAWW-Q397 & $09 / 18 / 97$ & 1,3,5-TRINITROBENZENE & ND & 0.030 & $U G / L$ & USATHAMA \\
\hline GW-RAWW-Q397 & $09 / 18 / 97$ & 1,3-DINITROBENZENE & ND & 0.090 & UG/L & USATHAMA \\
\hline GW-RAWW-Q397 & $09 / 18 / 97$ & $2,4,6$-TR INITROTOLUENE & ND & 0.030 & UG $/ L$ & USATHAMA \\
\hline GW-RAWW-Q397 & $09 / 18 / 97$ & 2,4-DINITROTOLUENE & ND & 0.030 & $\mathrm{UG} / \mathrm{L}$ & USATHAMA \\
\hline GW-RAWW-Q397 & $09 / 18 / 97$ & 2,6-DINITROTOLUENE & ND & 0.010 & UG/L & USATHAMA \\
\hline GW-RAWW-0397 & $09 / 18 / 97$ & NI TROBENZENE & ND & 0.030 & UG/L & USATHAMA \\
\hline GW-RMW1-0397 & $09 / 16 / 97$ & $1,3,5$-TRINITROBENZENE & ND & 0.030 & UG/L & USATHAMA \\
\hline GW-RMW1-0397 & $09 / 16 / 97$ & 1,3-DINITROBENZENE & ND & 0.090 & UG/L & USATHAMA \\
\hline GW-RMW1-0397 & $09 / 16 / 97$ & 2,4,6-TRINITROTOLUENE & ND & 0.030 & $U G / L$ & USATHAMA \\
\hline GW-RMW1-Q397 & $09 / 16 / 97$ & 2,4-DINITROTOLUENE & ND & 0.030 & UG/L & USATHAMA \\
\hline GW-RMW1-0397 & $09 / 16 / 97$ & 2,6-DINITROTOLUENE & ND & 0.010 & UG/L & USATHAMA \\
\hline
\end{tabular}




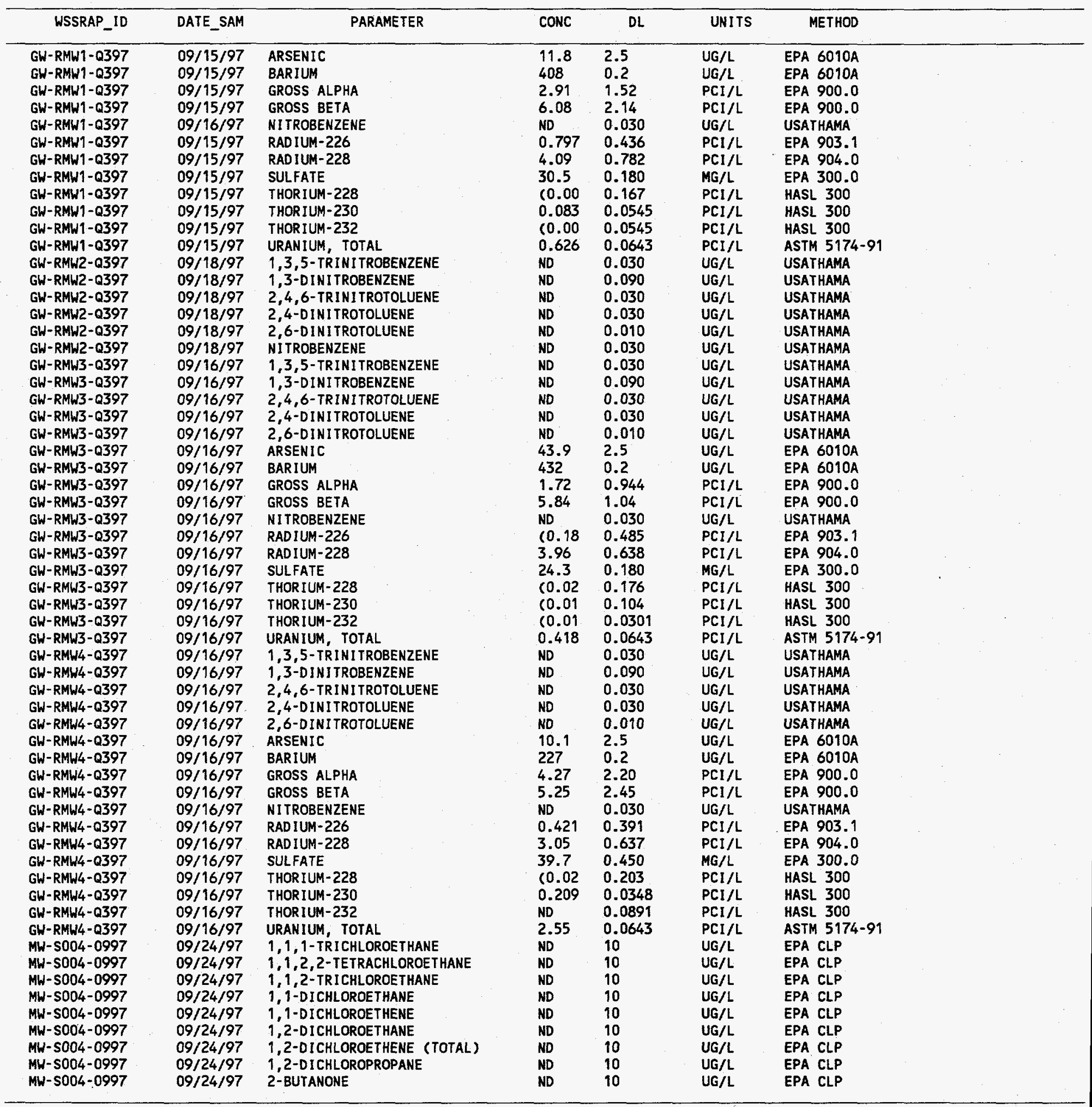




\begin{tabular}{|c|c|c|c|c|c|c|}
\hline WSSRAP_ID & DATE_SAM & PARAMETER & CONC & $D L$ & UNITS & METHOD \\
\hline 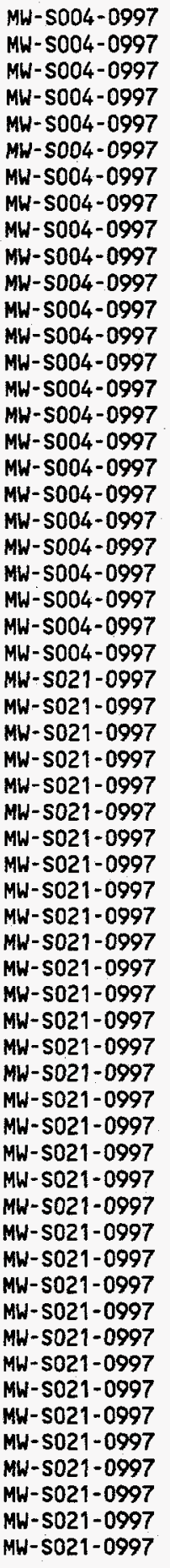 & $\begin{array}{l}09 / 24 / 97 \\
09 / 24 / 97 \\
09 / 24 / 97 \\
09 / 24 / 97 \\
09 / 24 / 97 \\
09 / 24 / 97 \\
09 / 24 / 97 \\
09 / 24 / 97 \\
09 / 24 / 97 \\
09 / 24 / 97 \\
09 / 24 / 97 \\
09 / 24 / 97 \\
09 / 24 / 97 \\
09 / 24 / 97 \\
09 / 24 / 97 \\
09 / 24 / 97 \\
09 / 24 / 97 \\
09 / 24 / 97 \\
09 / 24 / 97 \\
09 / 24 / 97 \\
09 / 24 / 97 \\
09 / 24 / 97 \\
09 / 24 / 97 \\
09 / 24 / 97 \\
09 / 24 / 97 \\
09 / 24 / 97 \\
09 / 24 / 97 \\
09 / 24 / 97 \\
09 / 24 / 97 \\
09 / 24 / 97 \\
09 / 24 / 97 \\
09 / 24 / 97 \\
09 / 24 / 97 \\
09 / 24 / 97 \\
09 / 24 / 97 \\
09 / 24 / 97 \\
09 / 24 / 97 \\
09 / 24 / 97 \\
09 / 24 / 97 \\
09 / 24 / 97 \\
09 / 24 / 97 \\
09 / 24 / 97 \\
09 / 24 / 97 \\
09 / 24 / 97 \\
09 / 24 / 97 \\
09 / 24 / 97 \\
09 / 24 / 97 \\
09 / 24 / 97 \\
09 / 24 / 97 \\
09 / 24 / 97 \\
09 / 24 / 97 \\
09 / 24 / 97 \\
09 / 24 / 97 \\
09 / 24 / 97 \\
09 / 24 / 97 \\
09 / 24 / 97 \\
09 / 24 / 97 \\
09 / 24 / 97 \\
09 / 24 / 97\end{array}$ & 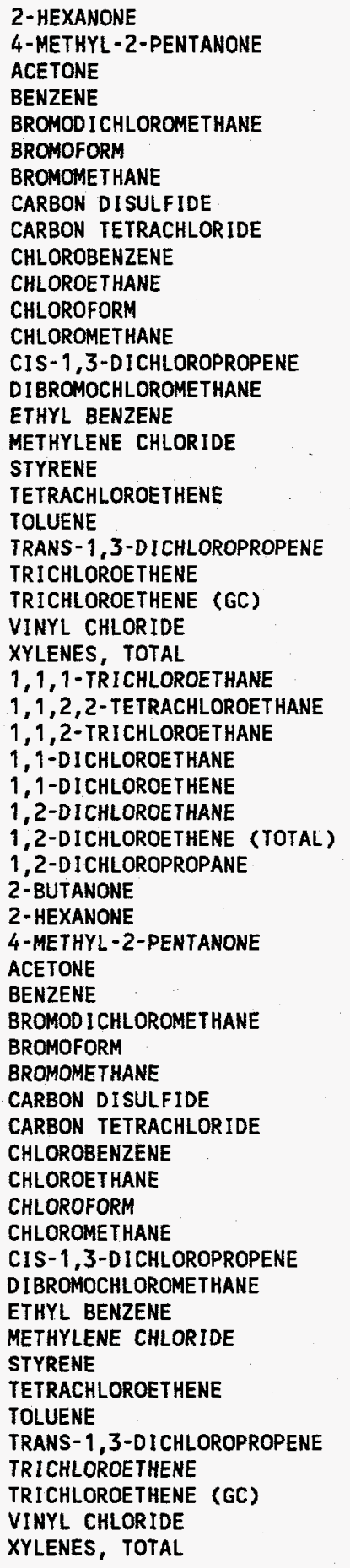 & $\begin{array}{l}\text { ND } \\
\text { ND } \\
\text { ND } \\
\text { ND } \\
\text { ND } \\
\text { ND } \\
\text { ND } \\
\text { ND } \\
\text { ND } \\
\text { ND } \\
\text { ND } \\
\text { ND } \\
\text { ND } \\
\text { ND } \\
\text { ND } \\
\text { ND } \\
\text { ND } \\
\text { ND } \\
\text { ND } \\
\text { ND } \\
\text { ND } \\
\text { ND } \\
\text { ND } \\
\text { ND } \\
\text { ND } \\
\text { ND } \\
\text { ND } \\
\text { ND } \\
\text { ND } \\
\text { ND } \\
\text { ND } \\
\text { C9 } \\
\text { ND } \\
\text { ND } \\
\text { ND } \\
\text { ND } \\
\text { ND } \\
\text { ND } \\
\text { ND } \\
\text { ND } \\
\text { ND } \\
\text { ND } \\
\text { ND } \\
\text { ND } \\
\text { ND } \\
\text { ND } \\
\text { ND } \\
\text { ND } \\
\text { ND } \\
\text { ND } \\
\text { ND } \\
\text { ND } \\
\text { ND } \\
\text { ND } \\
\text { ND } \\
\text { ND } \\
\text { NO } \\
\text { ND } \\
\text { ND }\end{array}$ & $\begin{array}{l}10 \\
10 \\
10 \\
10 \\
10 \\
10 \\
10 \\
10 \\
10 \\
10 \\
10 \\
10 \\
10 \\
10 \\
10 \\
10 \\
10 \\
10 \\
10 \\
10 \\
10 \\
10 \\
1.0 \\
10 \\
10 \\
10 \\
10 \\
10 \\
10 \\
10 \\
10 \\
10 \\
10 \\
10 \\
10 \\
10 \\
10 \\
10 \\
10 \\
10 \\
10 \\
10 \\
10 \\
10 \\
10 \\
10 \\
10 \\
10 \\
10 \\
10 \\
10 \\
10 \\
10 \\
10 \\
10 \\
100 \\
100 \\
10 \\
10\end{array}$ & 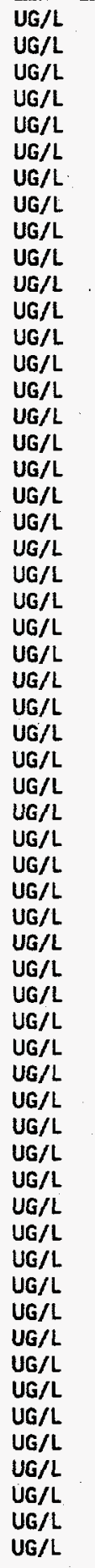 & 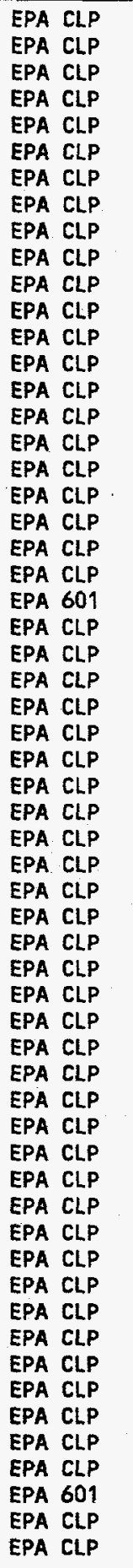 \\
\hline
\end{tabular}


SPRINGS 


\begin{tabular}{|c|c|c|c|c|c|c|}
\hline WSSRAP_ID & DATE_SAM & PARAMETER & CONC & $\mathrm{DL}$ & UNITS & METHOO \\
\hline $\begin{array}{l}\text { SP-6301-0997-L } \\
\text { SP-6301-0997-L } \\
\text { SP-6301-0997-L } \\
\text { SP-6301-0997-L } \\
\text { SP-6301-0997-L } \\
\text { SP-6301-0997-L } \\
\text { SP-6301-0997-L } \\
\text { SP-6301-0997-L } \\
\text { SP-6301-0997-L } \\
\text { SP-6301-0997-L } \\
\text { SP-6301-0997-L } \\
\text { SP-6301-0997-L } \\
\text { SP-6301-0997-L } \\
\text { SP-6301-0997-L } \\
\text { SP-6301-0997-L } \\
\text { SP-6301-0997-L } \\
\text { SP-6301-0997-L } \\
\text { SP-6301-0997-L } \\
\text { SP-6301-0997-L } \\
\text { SP-6301-0997-L } \\
\text { SP-6301-0997-L } \\
\text { SP-6301-0997-L } \\
\text { SP-6301-0997-L } \\
\text { SP-6301-0997-L } \\
\text { SP-6301-0997-L } \\
\text { SP-6301-0997-L } \\
\text { SP-6301-0497-H } \\
\text { SP-6301-1097-L- } \\
\text { SP-6301-1097-L- } \\
\text { SP-6301-1097-L- } \\
\text { SP-6301-1097-L- } \\
\text { SP-6301-1097-L- } \\
\text { SP-6301-1097-L- } \\
\text { SP-6301-1097-L- } \\
\text { SP-6301-1097-L- } \\
\text { SP-6301-0997-L } \\
\text { SP-6301-Q497-H } \\
\text { SP-6301-0997-L } \\
\text { SP-6301-1097-L- } \\
\text { SP-6301-1097-L- } \\
\text { SP-6301-1097-L- } \\
\text { SP-6301-1097-L- } \\
\text { SP-6301-1097-L- } \\
\text { SP-6301-1097-L- } \\
\text { SP-6301-1097-L- } \\
\text { SP-6301-1097-L- } \\
\text { SP-6301-1097-L- } \\
\text { SP-6301-1097-L- } \\
\text { SP-6301-1097-L- } \\
\text { SP-6301-1097-L- } \\
\text { SP-6301-0997-L } \\
\text { SP-6301-0997-L } \\
\text { SP-6301-0997-L } \\
\text { SP-6301-0997-L } \\
\text { SP-6301-0997-L } \\
\text { SP-6301-1097-L- } \\
\text { SP-6301-1097-L- } \\
\text { SP-6301-1097-L- }\end{array}$ & $\begin{array}{l}09 / 29 / 97 \\
09 / 29 / 97 \\
09 / 29 / 97 \\
09 / 29 / 97 \\
09 / 29 / 97 \\
09 / 29 / 97 \\
09 / 29 / 97 \\
09 / 29 / 97 \\
09 / 29 / 97 \\
09 / 29 / 97 \\
09 / 29 / 97 \\
09 / 29 / 97 \\
09 / 29 / 97 \\
09 / 29 / 97 \\
09 / 29 / 97 \\
09 / 29 / 97 \\
09 / 29 / 97 \\
09 / 29 / 97 \\
09 / 29 / 97 \\
09 / 29 / 97 \\
09 / 29 / 97 \\
09 / 29 / 97 \\
09 / 29 / 97 \\
09 / 29 / 97 \\
09 / 29 / 97 \\
09 / 29 / 97 \\
11 / 06 / 97 \\
10 / 15 / 97 \\
10 / 15 / 97 \\
10 / 15 / 97 \\
10 / 15 / 97 \\
10 / 15 / 97 \\
10 / 15 / 97 \\
10 / 15 / 97 \\
10 / 15 / 97 \\
09 / 29 / 97 \\
11 / 06 / 97 \\
09 / 29 / 97 \\
10 / 15 / 97 \\
10 / 15 / 97 \\
10 / 15 / 97 \\
10 / 15 / 97 \\
10 / 15 / 97 \\
10 / 15 / 97 \\
10 / 15 / 97 \\
10 / 15 / 97 \\
10 / 15 / 97 \\
10 / 15 / 97 \\
10 / 15 / 97 \\
10 / 15 / 97 \\
09 / 29 / 97 \\
09 / 29 / 97 \\
09 / 29 / 97 \\
09 / 29 / 97 \\
09 / 29 / 97 \\
10 / 15 / 97 \\
10 / 15 / 97 \\
10 / 15 / 97\end{array}$ & 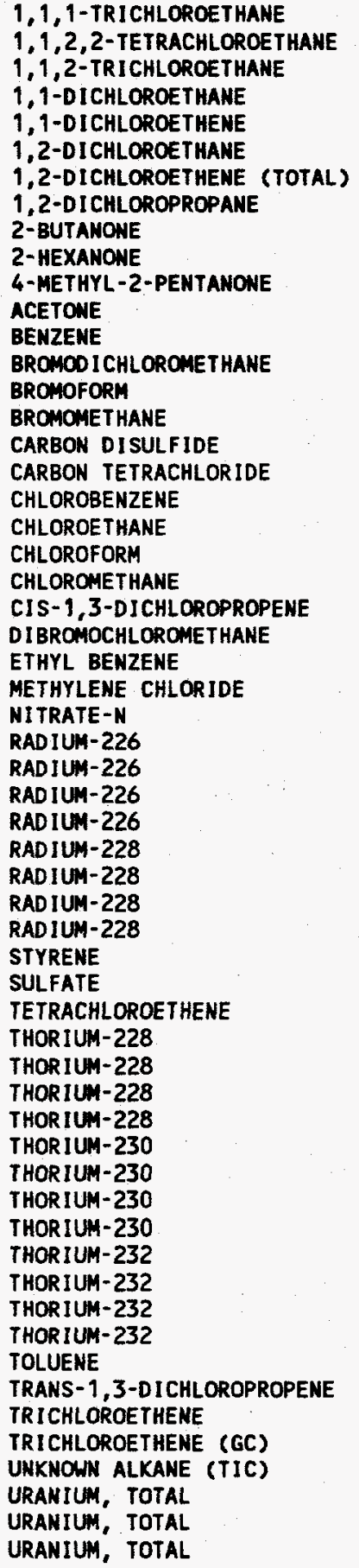 & $\begin{array}{l}\text { ND } \\
\text { ND } \\
\text { ND } \\
\text { ND } \\
\text { ND } \\
\text { ND } \\
\text { ND } \\
\text { ND } \\
\text { ND } \\
\text { ND } \\
\text { ND } \\
\text { ND } \\
\text { ND } \\
\text { ND } \\
\text { ND } \\
\text { ND } \\
\text { ND } \\
\text { ND } \\
\text { ND } \\
\text { ND } \\
\text { ND } \\
\text { ND } \\
\text { ND } \\
\text { ND } \\
\text { ND } \\
\text { ND } \\
2.83 \\
\text { CO.12 } \\
\text { ND } \\
\text { ND } \\
\text { Co.34 } \\
\text { C0.77 } \\
\text { C0.52 } \\
\text { C0.59 } \\
\text { CD.24 } \\
\text { ND } \\
44.5 \\
\text { ND } \\
\text { C0.03 } \\
\text { ND } \\
\text { C0.12 } \\
1.08 \\
\text { ND } \\
1.3 \\
\text { ND } \\
\text { ND } \\
\text { ND } \\
0.5 \\
\text { C0.07 } \\
0.53 \\
\text { ND } \\
\text { ND } \\
\text { ND } \\
\text { ND } \\
2 \\
110 \\
110 \\
110 \\
\text { Do }\end{array}$ & $\begin{array}{l}10 \\
10 \\
10 \\
10 \\
10 \\
10 \\
10 \\
10 \\
10 \\
10 \\
10 \\
10 \\
10 \\
10 \\
10 \\
10 \\
10 \\
10 \\
10 \\
10 \\
10 \\
10 \\
10 \\
10 \\
10 \\
10 \\
0.225 \\
0.5 \\
0.5 \\
0.5 \\
0.5 \\
1.89 \\
1.89 \\
1.89 \\
1.89 \\
10 \\
2.5 \\
10 \\
0.93 \\
0.98 \\
0.81 \\
0.98 \\
0.91 \\
0.96 \\
0.81 \\
0.89 \\
0.21 \\
0.25 \\
0.21 \\
0.24 \\
10 \\
10 \\
10 \\
1.0 \\
0.00 \\
0.677 \\
0.677 \\
0.677\end{array}$ & $\begin{array}{l}\text { UG/L } \\
U G / L \\
U G / L \\
U G / L \\
U G / L \\
U G / L \\
U G / L \\
U G / L \\
U G / L \\
U G / L \\
U G / L \\
U G / L \\
U G / L \\
U G / L \\
U G / L \\
U G / L \\
U G / L \\
U G / L \\
U G / L \\
U G / L \\
U G / L \\
U G / L \\
U G / L \\
U G / L \\
U G / L \\
U G / L \\
M G / L \\
P C I / L \\
P C I / L \\
P C I / L \\
P C I / L \\
P C I / L \\
P C I / L \\
P C I / L \\
P C I / L \\
U G / L \\
M G / L \\
U G / L \\
P C I / L \\
P C I / L \\
P C I / L \\
P C I / L \\
P C I / L \\
P C I / L \\
P C I / L \\
P C I / L \\
P C I / L \\
P C I / L \\
P C I / L \\
P C I / L \\
U G / L \\
U G / L \\
U G / L \\
U G / L \\
U G / L \\
P C I / L \\
P C I / L \\
P C I / L\end{array}$ & $\begin{array}{l}\text { EPA CLP } \\
\text { EPA CLP } \\
\text { EPA CLP } \\
\text { EPA CLP } \\
\text { EPA CLP } \\
\text { EPA CLP } \\
\text { EPA CLP } \\
\text { EPA CLP } \\
\text { EPA CLP } \\
\text { EPA CLP } \\
\text { EPA CLP } \\
\text { EPA CLP } \\
\text { EPA CLP } \\
\text { EPA CLP } \\
\text { EPA CLP } \\
\text { EPA CLP } \\
\text { EPA CLP } \\
\text { EPA CLP } \\
\text { EPA CLP } \\
\text { EPA CLP } \\
\text { EPA CLP } \\
\text { EPA CLP } \\
\text { EPA CLP } \\
\text { EPA CLP } \\
\text { EPA CLP } \\
\text { EPA CLP } \\
\text { EPA } 300.0 \\
\text { SM-705 } \\
\text { SM-705 } \\
\text { SM-705 } \\
\text { SM-705 } \\
\text { PERC/BROOKS } \\
\text { PERC/BROOKS } \\
\text { PERC/BRO0KS } \\
\text { PERC/BROOKS } \\
\text { EPA CLP } \\
\text { EPA } 300.0 \\
\text { EPA CLP } \\
\text { USAEC } \\
\text { USAEC } \\
\text { USAEC } \\
\text { USAEC } \\
\text { USAEC } \\
\text { USAEC } \\
\text { USAEC } \\
\text { USAEC } \\
\text { USAEC } \\
\text { USAEC } \\
\text { USAEC } \\
\text { USAEC } \\
\text { EPA CLP } \\
\text { EPA CLP } \\
\text { EPA CLP } \\
\text { EPA } 601 \\
\text { EPA CLP } \\
\text { ASTM D2907 } \\
\text { ASTM D2907 } \\
\text { ASTM D2907 }\end{array}$ \\
\hline
\end{tabular}




\begin{tabular}{|c|c|c|c|c|c|c|}
\hline WSSRAP_ID & DATE_SAM & PARAMETER & CONC & DL & UNITS & METHOD \\
\hline $\begin{array}{l}\text { SP-6301-1097-L- } \\
\text { SP-6301-0997-L } \\
\text { SP-6301-0997-L } \\
\text { SP-6303-0997-L } \\
\text { SP-6303-0997-L } \\
\text { SP-6303-0997-L } \\
\text { SP-6303-0997-L } \\
\text { SP-6303-0997-L } \\
\text { SP- } 6303-0997-L \\
\text { SP-6303-0997-L } \\
\text { SP-6303-0997-L } \\
\text { SP-6303-0997-L } \\
\text { SP-6303-0997-L } \\
\text { SP-6303-0997-L } \\
\text { SP-6303-0997-L } \\
\text { SP-6303-0997-L } \\
\text { SP-6303-0997-L } \\
\text { SP-6303-0997-L } \\
\text { SP-6303-0997-L } \\
\text { SP-6303-0997-L } \\
\text { SP-6303-0997-L } \\
\text { SP-6303-0997-L } \\
\text { SP-6303-0997-L } \\
\text { SP-6303-0997-L } \\
\text { SP-6303-0997-L } \\
\text { SP-6303-0997-L } \\
\text { SP-6303-0997-L } \\
\text { SP-6303-0997-L } \\
\text { SP-6303-0997-L } \\
\text { SP-6303-0997-L } \\
\text { SP-6303-0997-L } \\
\text { SP-6303-0997-L } \\
\text { SP-6303-0997-L } \\
\text { SP-6303-0997-L } \\
\text { SP-6303-0997-L } \\
\text { SP-6303-0997-L } \\
\text { SP-6303-0997-L } \\
\text { SP-6303-0997-L }\end{array}$ & $\begin{array}{l}10 / 15 / 97 \\
09 / 29 / 97 \\
09 / 29 / 97 \\
09 / 29 / 97 \\
09 / 29 / 97 \\
09 / 29 / 97 \\
09 / 29 / 97 \\
09 / 29 / 97 \\
09 / 29 / 97 \\
09 / 29 / 97 \\
09 / 29 / 97 \\
09 / 29 / 97 \\
09 / 29 / 97 \\
09 / 29 / 97 \\
09 / 29 / 97 \\
09 / 29 / 97 \\
09 / 29 / 97 \\
09 / 29 / 97 \\
09 / 29 / 97 \\
09 / 29 / 97 \\
09 / 29 / 97 \\
09 / 29 / 97 \\
09 / 29 / 97 \\
09 / 29 / 97 \\
09 / 29 / 97 \\
09 / 29 / 97 \\
09 / 29 / 97 \\
09 / 29 / 97 \\
09 / 29 / 97 \\
09 / 29 / 97 \\
09 / 29 / 97 \\
09 / 29 / 97 \\
09 / 29 / 97 \\
09 / 29 / 97 \\
09 / 29 / 97 \\
09 / 29 / 97 \\
09 / 29 / 97 \\
09 / 29 / 97\end{array}$ & 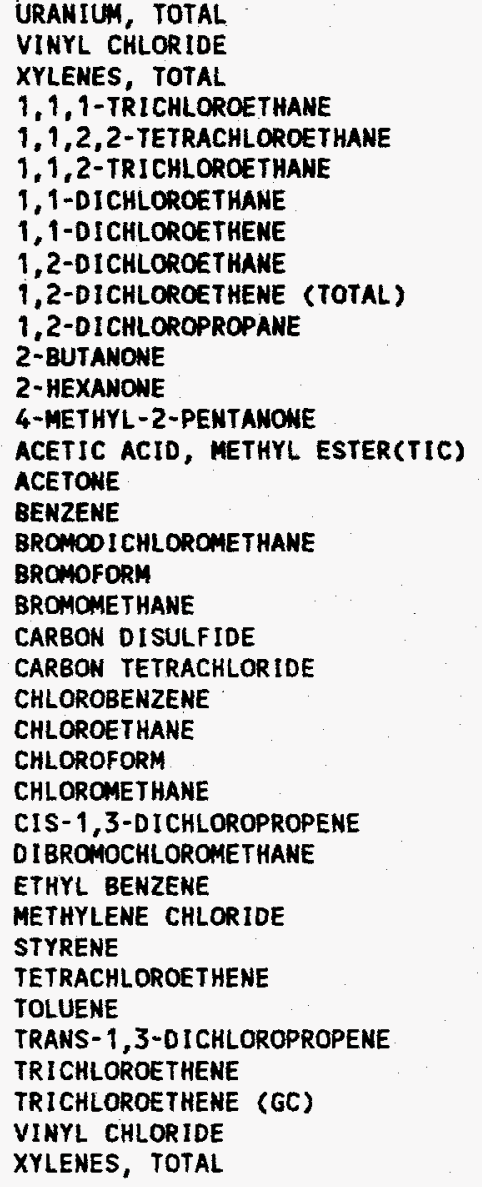 & $\begin{array}{l}\text { No } \\
\text { ND } \\
\text { ND } \\
\text { ND } \\
\text { ND } \\
\text { ND } \\
\text { ND } \\
\text { ND } \\
\text { ND } \\
\text { ND } \\
\text { ND } \\
\text { ND } \\
\text { ND } \\
\text { ND } \\
\text { ND } \\
\text { ND } \\
\text { ND } \\
\text { ND } \\
\text { ND } \\
\text { ND } \\
\text { ND } \\
\text { ND } \\
\text { ND } \\
\text { ND } \\
\text { ND } \\
\text { ND } \\
\text { ND } \\
\text { ND } \\
\text { ND } \\
\text { ND } \\
\text { ND } \\
\text { ND } \\
\text { ND } \\
\text { ND }\end{array}$ & $\begin{array}{l}0.677 \\
10 \\
10 \\
10 \\
10 \\
10 \\
10 \\
10 \\
10 \\
10 \\
10 \\
10 \\
10 \\
10 \\
0.00 \\
10 \\
10 \\
10 \\
10 \\
10 \\
10 \\
10 \\
10 \\
10 \\
10 \\
10 \\
10 \\
10 \\
10 \\
10 \\
10 \\
10 \\
10 \\
10 \\
10 \\
1.0 \\
10 \\
10 \\
\end{array}$ & $\begin{array}{l}P C I / L \\
U G / L \\
U G / L \\
U G / L \\
U G / L \\
U G / L \\
U G / L \\
U G / L \\
U G / L \\
U G / L \\
U G / L \\
U G / L \\
U G / L \\
U G / L \\
U G / L \\
U G / L \\
U G / L \\
U G / L \\
U G / L \\
U G / L \\
U G / L \\
U G / L \\
U G / L \\
U G / L \\
U G / L \\
U G / L \\
U G / L \\
U G / L \\
U G / L \\
U G / L \\
U G / L \\
U G / L \\
U G / L \\
U G / L \\
U G / L \\
U G / L \\
U G / L \\
U G / L\end{array}$ & $\begin{array}{l}\text { ASTM } 02907 \\
\text { EPA CLP } \\
\text { EPA CLP } \\
\text { EPA CLP } \\
\text { EPA CLP } \\
\text { EPA CLP } \\
\text { EPA CLP } \\
\text { EPA CLP } \\
\text { EPA CLP } \\
\text { EPA CLP } \\
\text { EPA CLP } \\
\text { EPA CLP } \\
\text { EPA CLP } \\
\text { EPA CLP } \\
\text { EPA CLP } \\
\text { EPA CLP } \\
\text { EPA CLP } \\
\text { EPA CLP } \\
\text { EPA CLP } \\
\text { EPA CLP } \\
\text { EPA CLP } \\
\text { EPA CLP } \\
\text { EPA CLP } \\
\text { EPA CLP } \\
\text { EPA CLP } \\
\text { EPA CLP } \\
\text { EPA CLP } \\
\text { EPA CLP } \\
\text { EPA CLP } \\
\text { EPA CLP } \\
\text { EPA CLP } \\
\text { EPA CLP } \\
\text { EPA CLP } \\
\text { EPA CLP } \\
\text { EPA CLP } \\
\text { EPA } 601 \\
\text { EPA CLP } \\
\text { EPA CLP }\end{array}$ \\
\hline
\end{tabular}


SURFACE WATER 
SURFACE WATER

DATA MERGED DURING FOURTH QUARTER 1997

\begin{tabular}{|c|c|c|c|c|c|c|}
\hline USSRAP_ID & DATE_SAM & PARAMETER & CONC & DL & UNITS & METHOD \\
\hline $\begin{array}{l}\text { SW-1001A-102797 } \\
\text { SW-1001A-102797 } \\
\text { SW-1001A-102797 } \\
\text { SW-1001A-102797 } \\
\text { SW-1001A-102797 } \\
\text { SW-1001A-102797 } \\
\text { SW-1001A-102797 } \\
\text { SW-1001A-102797 } \\
\text { SW-1001A-102797 } \\
\text { SW-1001A-102797 } \\
\text { SW-1001A-102797 } \\
\text { SW-1001A-102797 } \\
\text { SW-1001A-102797 } \\
\text { SW-1001A-102797 } \\
\text { SW-1001A-102797 } \\
\text { SW-1001A-102797 } \\
\text { SW-1001A-102797 } \\
\text { SW-1001A-102797 } \\
\text { SW-1001A-102797 } \\
\text { SW-1003-B597 } \\
\text { SW-1003-B597 } \\
\text { SW-1003-B597 } \\
\text { SW-1003-B597 } \\
\text { SW-1003-B597 } \\
\text { SW-1003-B597 } \\
\text { SW-1004-B597 } \\
\text { SW-1004-8597 } \\
\text { SW-1004-B597 } \\
\text { SW-1004-B597 } \\
\text { SW-1004-B597 } \\
\text { SW-1004-B597 } \\
\text { SW-1005-B597 } \\
\text { SW-1005-B597 } \\
\text { SW-1005-B597 } \\
\text { SW-1005-B597 } \\
\text { SW-1005-B597 } \\
\text { SW-1005-B597 } \\
\text { SW-2010-110597 } \\
\text { SW-2010-110597 } \\
\text { SW-2010-110597 } \\
\text { SW-2010-110597 } \\
\text { SW-2010-110597 } \\
\text { SW-2010-110597 } \\
\text { SW-2010-110597 } \\
\text { SW-2015-102897 } \\
\text { SW-2015-102897 } \\
\text { SW-2015-1028977 } \\
\text { SW-2015-102897 } \\
\text { SW-2015-102897 } \\
\text { SW-2015-102897 } \\
\text { SW-2015-1028977 } \\
\text { SW-2015-102897 } \\
\text { SW-2015-102897 } \\
\text { SW-2015-102897 } \\
\text { SW-2015-102897 } \\
\text { SW-2015-102897 } \\
\text { SW-2015-1028977 } \\
\text { SW-2015-102897 }\end{array}$ & $\begin{array}{l}10 / 27 / 97 \\
10 / 27 / 97 \\
10 / 27 / 97 \\
10 / 27 / 97 \\
10 / 27 / 97 \\
10 / 27 / 97 \\
10 / 27 / 97 \\
10 / 27 / 97 \\
10 / 27 / 97 \\
10 / 27 / 97 \\
10 / 27 / 97 \\
10 / 27 / 97 \\
10 / 27 / 97 \\
10 / 27 / 97 \\
10 / 27 / 97 \\
10 / 27 / 97 \\
10 / 27 / 97 \\
10 / 27 / 97 \\
10 / 27 / 97 \\
10 / 13 / 97 \\
10 / 13 / 97 \\
10 / 13 / 97 \\
10 / 13 / 97 \\
10 / 13 / 97 \\
10 / 13 / 97 \\
10 / 13 / 97 \\
10 / 13 / 97 \\
10 / 13 / 97 \\
10 / 13 / 97 \\
10 / 13 / 97 \\
10 / 13 / 97 \\
10 / 13 / 97 \\
10 / 13 / 97 \\
10 / 13 / 97 \\
10 / 13 / 97 \\
10 / 13 / 97 \\
10 / 13 / 97 \\
11 / 05 / 97 \\
11 / 05 / 97 \\
11 / 05 / 97 \\
11 / 05 / 97 \\
11 / 05 / 97 \\
11 / 05 / 97 \\
11 / 05 / 97 \\
10 / 28 / 97 \\
10 / 28 / 97 \\
10 / 28 / 97 \\
10 / 28 / 97 \\
10 / 28 / 97 \\
10 / 28 / 97 \\
10 / 28 / 97 \\
10 / 28 / 97 \\
10 / 28 / 97 \\
10 / 28 / 97 \\
10 / 28 / 97 \\
10 / 28 / 97 \\
10 / 28 / 97 \\
10 / 28 / 97\end{array}$ & 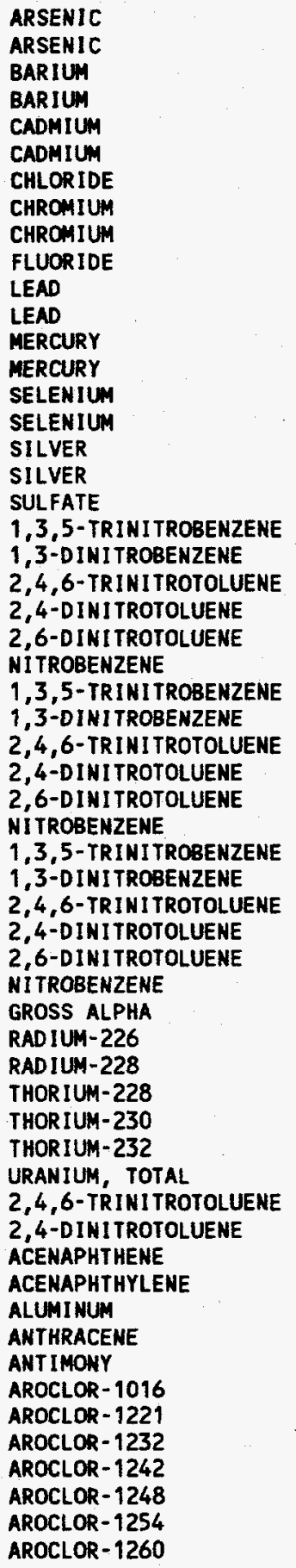 & $\begin{array}{l}\text { ND } \\
\text { ND } \\
110 \\
104 \\
\text { ND } \\
2.7 \\
8.29 \\
\text { ND } \\
\text { ND } \\
\text { O.11 } \\
\text { ND } \\
\text { ND } \\
\text { ND } \\
\text { ND } \\
\text { ND } \\
\text { ND } \\
\text { ND } \\
\text { 27.4 } \\
\text { ND } \\
\text { ND } \\
\text { ND } \\
\text { ND } \\
\text { ND } \\
\text { ND } \\
\text { ND } \\
\text { ND } \\
\text { ND } \\
\text { ND } \\
\text { ND } \\
\text { ND } \\
\text { ND } \\
\text { ND } \\
\text { ND } \\
\text { ND } \\
\text { ND } \\
\text { ND } \\
231 \\
1.48 \\
3.02 \\
\text { O.23 } \\
2.73 \\
0.457 \\
183 \\
\text { ND } \\
\text { ND } \\
\text { ND } \\
\text { ND } \\
\text { 338 } \\
\text { ND } \\
\text { ND } \\
\text { ND } \\
\text { ND } \\
\text { ND } \\
\text { ND } \\
\text { ND } \\
\text { ND }\end{array}$ & 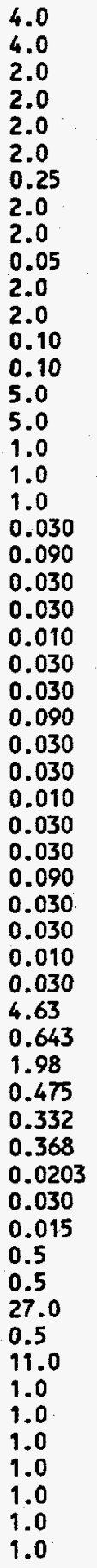 & 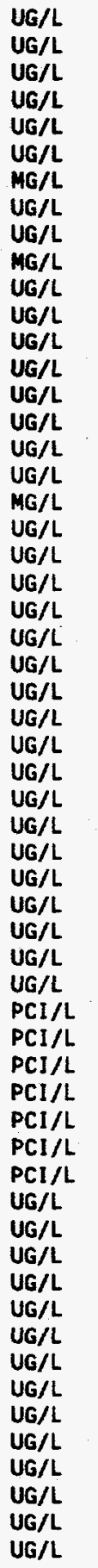 & $\begin{array}{l}\text { EPA CLP } \\
\text { EPA CLP } \\
\text { EPA CLP } \\
\text { EPA CLP } \\
\text { EPA CLP } \\
\text { EPA CLP } \\
\text { EPA } 300.0 \\
\text { EPA CLP } \\
\text { EPA CLP } \\
\text { EPA } 340 \\
\text { EPA CLP } \\
\text { EPA CLP } \\
\text { EPA CLP } \\
\text { EPA CLP } \\
\text { EPA CLP } \\
\text { EPA CLP } \\
\text { EPA CLP } \\
\text { EPA CLP } \\
\text { EPA } 300.0 \\
\text { USATHAMA } \\
\text { USATHAMA } \\
\text { USATHAMA } \\
\text { USATHAMA } \\
\text { USATHAMA } \\
\text { USATHAMA } \\
\text { USATHAMA } \\
\text { USATHAMA } \\
\text { USATHAMA } \\
\text { USATHAMA } \\
\text { USATHAMA } \\
\text { USATHAMA } \\
\text { USATHAMA } \\
\text { USATHAMA } \\
\text { USATHAMA } \\
\text { USATHAMA } \\
\text { USATHAMA } \\
\text { USATHAMA } \\
\text { EPA 900.0 } \\
\text { EPA 903.0 } \\
\text { EPA 904.0 } \\
\text { EML TH-01 } \\
\text { EML TH-01 } \\
\text { EML TH-01 } \\
\text { ASTM } 5174-91 \\
\text { EPA } 609 \\
\text { EPA 609 } \\
\text { EPA } 8310 \\
\text { EPA } 8310 \\
\text { EPA } 6010 A \\
\text { EPA } 8310 \\
\text { EPA } 6010 A \\
\text { EPA } 8081 \\
\text { EPA } 8081 \\
\text { EPA } 8081 \\
\text { EPA } 8081 \\
\text { EPA } 8081 \\
\text { EPA } 8081 \\
\text { EPA } 8081 \\
\end{array}$ \\
\hline
\end{tabular}




\begin{tabular}{|c|c|c|c|c|c|c|}
\hline USSRAP_ID & DATE_SAM & PARAMETER & CONC & $\mathrm{DL}$ & UNITS & METHOD \\
\hline 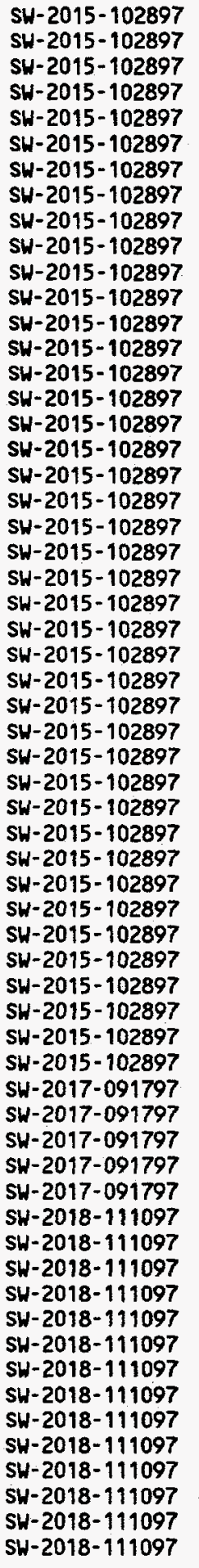 & $\begin{array}{l}10 / 28 / 97 \\
10 / 28 / 97 \\
10 / 28 / 97 \\
10 / 28 / 97 \\
10 / 28 / 97 \\
10 / 28 / 97 \\
10 / 28 / 97 \\
10 / 28 / 97 \\
10 / 28 / 97 \\
10 / 28 / 97 \\
10 / 28 / 97 \\
10 / 28 / 97 \\
10 / 28 / 97 \\
10 / 28 / 97 \\
10 / 28 / 97 \\
10 / 28 / 97 \\
10 / 28 / 97 \\
10 / 28 / 97 \\
10 / 28 / 97 \\
10 / 28 / 97 \\
10 / 28 / 97 \\
10 / 28 / 97 \\
10 / 28 / 97 \\
10 / 28 / 97 \\
10 / 28 / 97 \\
10 / 28 / 97 \\
10 / 28 / 97 \\
10 / 28 / 97 \\
10 / 28 / 97 \\
10 / 28 / 97 \\
10 / 28 / 97 \\
10 / 28 / 97 \\
10 / 28 / 97 \\
10 / 28 / 97 \\
10 / 28 / 97 \\
10 / 28 / 97 \\
10 / 28 / 97 \\
10 / 28 / 97 \\
10 / 28 / 97 \\
10 / 28 / 97 \\
10 / 28 / 97 \\
10 / 28 / 97 \\
09 / 17 / 97 \\
09 / 17 / 97 \\
09 / 17 / 97 \\
09 / 17 / 97 \\
09 / 17 / 97 \\
11 / 10 / 97 \\
11 / 10 / 97 \\
11 / 10 / 97 \\
11 / 10 / 97 \\
11 / 10 / 97 \\
11 / 10 / 97 \\
11 / 10 / 97 \\
11 / 10 / 97 \\
11 / 10 / 97 \\
11 / 10 / 97 \\
11 / 10 / 97 \\
11 / 10 / 97 \\
11 / 10 / 97 \\
11 / 10 / 97\end{array}$ & 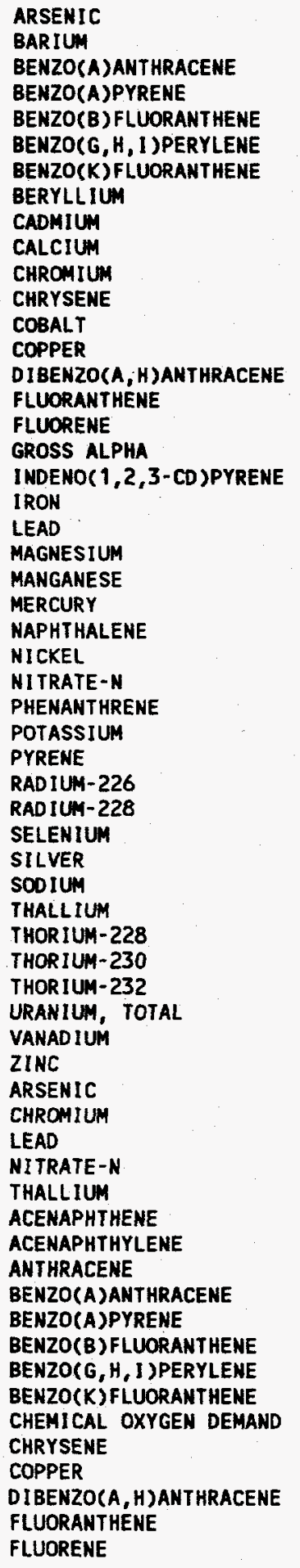 & $\begin{array}{l}\text { ND } \\
36.5 \\
\text { ND } \\
\text { ND } \\
\text { ND } \\
\text { ND } \\
\text { ND } \\
\text { ND } \\
\text { ND } \\
38700 \\
\text { ND } \\
\text { ND } \\
\text { ND } \\
2.3 \\
\text { ND } \\
\text { ND } \\
1.32 \\
128 \\
\text { ND } \\
366 \\
\text { ND } \\
10300 \\
9.6 \\
\text { ND } \\
\text { ND } \\
\text { ND } \\
0.798 \\
\text { ND } \\
2040 \\
\text { ND } \\
\text { C.40 } \\
\text { N1.37 } \\
\text { ND } \\
\text { ND } \\
12600 \\
\text { ND } \\
60.14 \\
0.487 \\
\text { ND } \\
161 \\
\text { ND } \\
47.3 \\
\text { ND } \\
2.3 \\
3.9 \\
9.30 \\
\text { ND } \\
\text { ND } \\
\text { ND } \\
\text { ND } \\
\text { ND } \\
0.002 \\
0.003 \\
\text { ND } \\
0.003 \\
163 \\
\text { ND } \\
7.50 \\
\text { ND } \\
0.012 \\
\text { ND }\end{array}$ & $\begin{array}{l}4.0 \\
2.0 \\
0.5 \\
0.5 \\
0.5 \\
0.5 \\
0.5 \\
1.0 \\
2.0 \\
13.0 \\
2.0 \\
0.5 \\
2.0 \\
2.0 \\
0.5 \\
0.5 \\
0.5 \\
1.22 \\
0.5 \\
61.0 \\
2.0 \\
28.0 \\
2.0 \\
0.10 \\
0.5 \\
2.0 \\
0.020 \\
0.5 \\
69.0 \\
0.5 \\
0.637 \\
2.35 \\
5.0 \\
1.0 \\
130 \\
7.0 \\
0.319 \\
0.294 \\
0.293 \\
7.45 \\
2.0 \\
6.0 \\
2.5 \\
0.5 \\
1.2 \\
0.0350 \\
2.4 \\
2.00 \\
1.50 \\
0.0990 \\
0.00140 \\
0.00122 \\
0.00150 \\
0.00398 \\
0.00120 \\
5.00 \\
0.0299 \\
2.00 \\
0.00250 \\
0.00198 \\
0.249\end{array}$ & $\begin{array}{l}U G / L \\
U G / L \\
U G / L \\
U G / L \\
U G / L \\
U G / L \\
U G / L \\
U G / L \\
U G / L \\
U G / L \\
U G / L \\
U G / L \\
U G / L \\
U G / L \\
U G / L \\
U G / L \\
U G / L \\
P C I / L \\
U G / L \\
U G / L \\
U G / L \\
U G / L \\
U G / L \\
U G / L \\
U G / L \\
U G / L \\
M G / L \\
U G / L \\
U G / L \\
U G / L \\
P C I / L \\
P C I / L \\
U G / L \\
U G / L \\
U G / L \\
U G / L \\
P C I / L \\
P C I / L \\
P C I / L \\
P C I / L \\
U G / L \\
U G / L \\
U G / L \\
U G / L \\
U G / L \\
M G / L \\
U G / L \\
U G / L \\
U G / L \\
U G / L \\
U G / L \\
U G / L \\
U G / L \\
U G / L \\
U G / L \\
M G / L \\
U G / L \\
U G / L \\
U G / L \\
U G / L \\
U G / L \\
\end{array}$ & 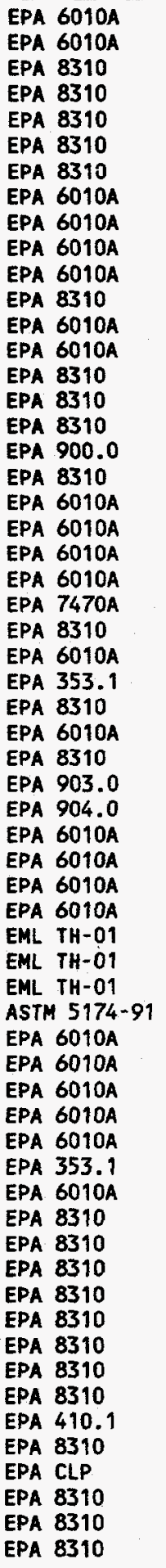 \\
\hline
\end{tabular}




\begin{tabular}{|c|c|c|c|c|c|c|}
\hline WSSRAP_ID & DATE_SAM & PARAMETER & CONC & DL & UNITS & METHOD \\
\hline $\begin{array}{l}\text { SW-2018-111097 } \\
\text { SW-2018-111097 } \\
\text { SW-2018-111097 } \\
\text { SW-2018-111097 } \\
\text { SW-2018- } 111097 \\
\text { SW-2018-111097 } \\
\text { SW-2020-091797 } \\
\text { SW-2020-091797 } \\
\text { SW-2020-091797 } \\
\text { SW-2020-091797 } \\
\text { SW-2020-091797 }\end{array}$ & $\begin{array}{l}11 / 10 / 97 \\
11 / 10 / 97 \\
11 / 10 / 97 \\
11 / 10 / 97 \\
11 / 10 / 97 \\
11 / 10 / 97 \\
09 / 17 / 97 \\
09 / 17 / 97 \\
09 / 17 / 97 \\
09 / 17 / 97 \\
09 / 17 / 97\end{array}$ & $\begin{array}{l}\text { INDENO }(1,2,3-C D) \text { PYRENE } \\
\text { NAPHTHALENE } \\
\text { NITRATE-N } \\
\text { PHENANTHRENE } \\
\text { PYRENE } \\
\text { ZINC } \\
\text { CALCIUN } \\
\text { CHLORIDE } \\
\text { FLUORIDE } \\
\text { SODIUM } \\
\text { SULFATE }\end{array}$ & $\begin{array}{l}\text { ND } \\
\text { NO } \\
7.57 \\
\text { ND } \\
\text { ND } \\
50.9 \\
57900 \\
535 \\
\text { ND } \\
26900 \\
45.1\end{array}$ & $\begin{array}{l}0.00248 \\
0.600 \\
0.500 \\
0.0744 \\
0.0278 \\
10.0 \\
2.8 \\
6.40 \\
0.0200 \\
7.1 \\
0.900\end{array}$ & $\begin{array}{l}\text { UG/L } \\
U G / L \\
\text { MG/L } \\
\text { UG/L } \\
\text { UG/L } \\
\text { UG/L } \\
\text { UG/L } \\
\text { MG/L } \\
\text { MG/L } \\
\text { UG/L } \\
\text { MG/L }\end{array}$ & $\begin{array}{ll}\text { EPA } & 8310 \\
\text { EPA } & 8310 \\
\text { EPA } & 353.2 \\
\text { EPA } & 8310 \\
\text { EPA } 8310 \\
\text { EPA CLP } \\
\text { EPA } 6010 A \\
\text { EPA } 300.0 \\
\text { EPA } 300.0 \\
\text { EPA } 6010 A \\
\text { EPA } 300.0\end{array}$ \\
\hline
\end{tabular}


SURFACE WATER DATA

FOURTH QUARTER 1997

KPA RESULTS

$\begin{array}{rrrlcccc}\text { SAMPLE ID } & \text { LOC. } & \text { DATE } & \text { PARAMETER } & \text { CONCENTRATION } & \text { ERROR } & \text { D.L. } & \text { UNITS } \\ \text { SW-2001-110697 } & 2001 & 11 / 6 / 97 & \text { URANIUM,TOTAL } & 3.228 & 0.106 & 0.7 & \text { PCI/L } \\ \text { SW-2002-110697 } & 2002 & 11 / 6 / 97 & \text { URANIUM,TOTAL } & 9.020 & 0.315 & 0.7 & \text { PCI/L } \\ \text { SW-2003-110697 } & 2003 & 11 / 6 / 97 & \text { URANIUM,TOTAL } & 10.442 & 0.368 & 0.7 & \text { PCI/L } \\ \text { SW-2004-110697 } & 2004 & 11 / 6 / 97 & \text { URANIUM,TOTAL } & 10.970 & 0.140 & 0.7 & \text { PCI/L } \\ \text { SW-2005-110697 } & 2005 & 11 / 6 / 97 & \text { URANIUM,TOTAL } & 8.783 & 0.310 & 0.7 & \text { PCI/L } \\ \text { SW-2012-110697 } & 2012 & 11 / 6 / 97 & \text { URANIUM,TOTAL } & 9.464 & 0.129 & 0.7 & \text { PCI/L } \\ \text { SW-2016-110697 } & 2016 & 11 / 6 / 97 & \text { URANIUM,TOTAL } & 2.632 & 0.080 & 0.7 & \text { PCI/L }\end{array}$


AIR MONITORING 


\section{Third Quarter 1997 Alpha Track Radon Monitoring Results}

\section{LOCATION ID}

Weldon Spring Quarry

RD-1002

RD-1005

Weldon Spring Chemical Plant

RD-2002
RD-2025
RD-2004
RD-2006
RD-2007

Weldon Spring Raffinate Pits

\section{$\operatorname{AVERAGE}(\mathrm{pCi} / 1)^{\otimes}$}

0.1

0.1

$\begin{array}{ll}0.1 & 0 \\ 0.2 & 3 \\ 0.2 & 3 \\ 0.1 & 0 \\ 0.1 & 0\end{array}$

$\begin{array}{lll}\text { RD-3001 } & 0.7 & 20 \\ \text { RD-3002 } & 0.6 & 17 \\ \text { RD-3003 } & 0.8 & 23 \\ \text { RD-3004 } & 0.1 & 0 \\ \text { RD-3005 } & 0.1 & 0 \\ \text { RD-3007 } & 14.7 & 487 \\ \text { RD-3008 } & 1.5 & 47 \\ \text { RD-3009 } & 0.2 & 3 \\ \text { RD-3010 } & 1.0 & 30 \\ \text { RD-3011 } & 0.2 & 3 \\ \text { RD-3012 } & 0.4 & 10 \\ \text { RD-3013 } & 3.3 & 70 \\ \text { RD-3014 } & 2.2 & 57 \\ \text { RD-3015 } & 1.8 & \end{array}$

Off-Site

$\mathrm{RD}-4001$

RD-4002

$\mathrm{RD}-4003$

$\mathrm{RD}-4005 *$

$\mathrm{RD}-4007$

$\mathrm{RD}-4009 *$

RD-4013

$\begin{array}{ll}0.1 & 0 \\ 0.1 & 0 \\ 0.2 & 3 \\ 0.1 & \text { N/A } \\ 0.1 & 0 \\ 0.1 & \text { N/A } \\ 0.5 & 13\end{array}$

* Denotes background location

N/A No percentage calculation performed on background locations

** Denotes the missing alpha track radon monitor

\# Derived Concentration Guide

@ Includes both radon and thoron 


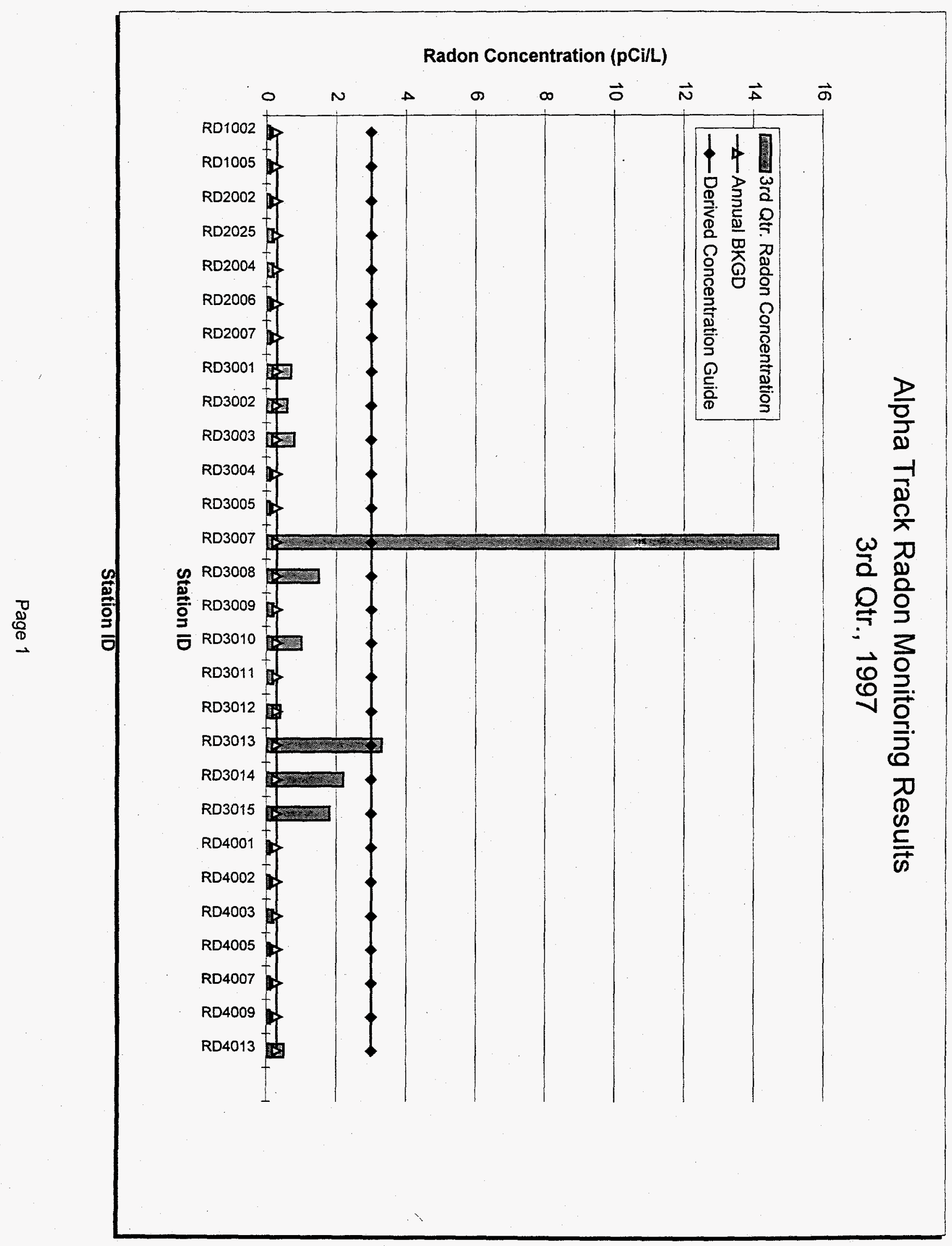




\section{Third Quarter 1997 Alpha Track Thoron Monitoring Results}

\section{LOCATION ID}

Weldon Spring Quarry

RD-1002

Weldon Spring Chemical Plant
AVERAGE(pCi/l)

0.1

0.0
0.2
0.3
0.1
0.0
PERCENT OF DCG

0

0

3

0
0

$\mathrm{RD}-2006$

RD-2007

Weldon Spring Raffinate Pits

$R D-3001$
$R D-3002$
$R D-3003$
$R D-3004$
$R D-3005$
$R D-3007$
$R D-3010$
$R D-3014$

1.1

0.8

1.2

0.0

0.1

20.0

1.0

2.7

Off-Site

$\mathrm{RD}-4001$

$\mathrm{RD}-4002$

$\mathrm{RD}-4003$

$R D-4005 *$

$\mathrm{RD}-4007$

$\mathrm{RD}-4009 *$

RD-4013

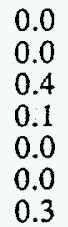

0
0
0
N/A
0
N/A
7

* Denotes background location

N/A No percentage calculation performed on background locations

** Denotes the missing alpha track radon monitor

\# Derived Concentration Guide 


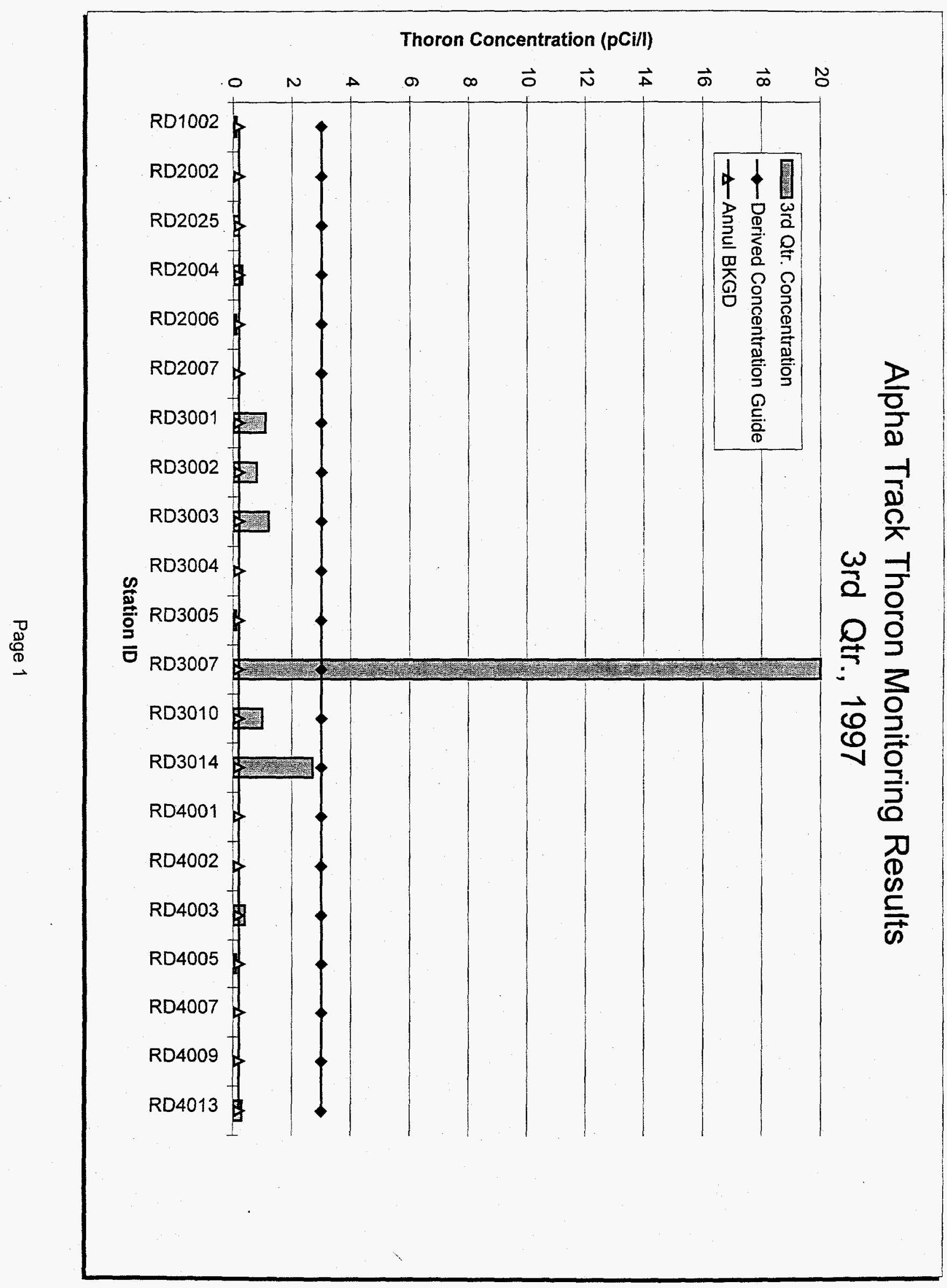




\section{Third Quarter 1997 Environmental TLD Monitoring Results}

LOCATION ID

Weldon Spring Quarry

TD-1002

Weldon Spring Chemical Plant

TD-2025

TD-2004

TD-2006

Weldon Spring Raffinate Pits

TD-3001

TD-3002

TD-3003

TD-3004

Off-Site

TD-4001

TD-4002

TD-4003

TD-4005*

TD 4007

TD-4009*
GAMMA EXPOSURE

(mR/Qtr)

14.6

15.6

16.3

14.1

16.9

20.0

21.2

15.7

14.8

12.2

11.3

13.3

14.3

13.4

* Denotes background location

** Denotes the missing TLDs. 


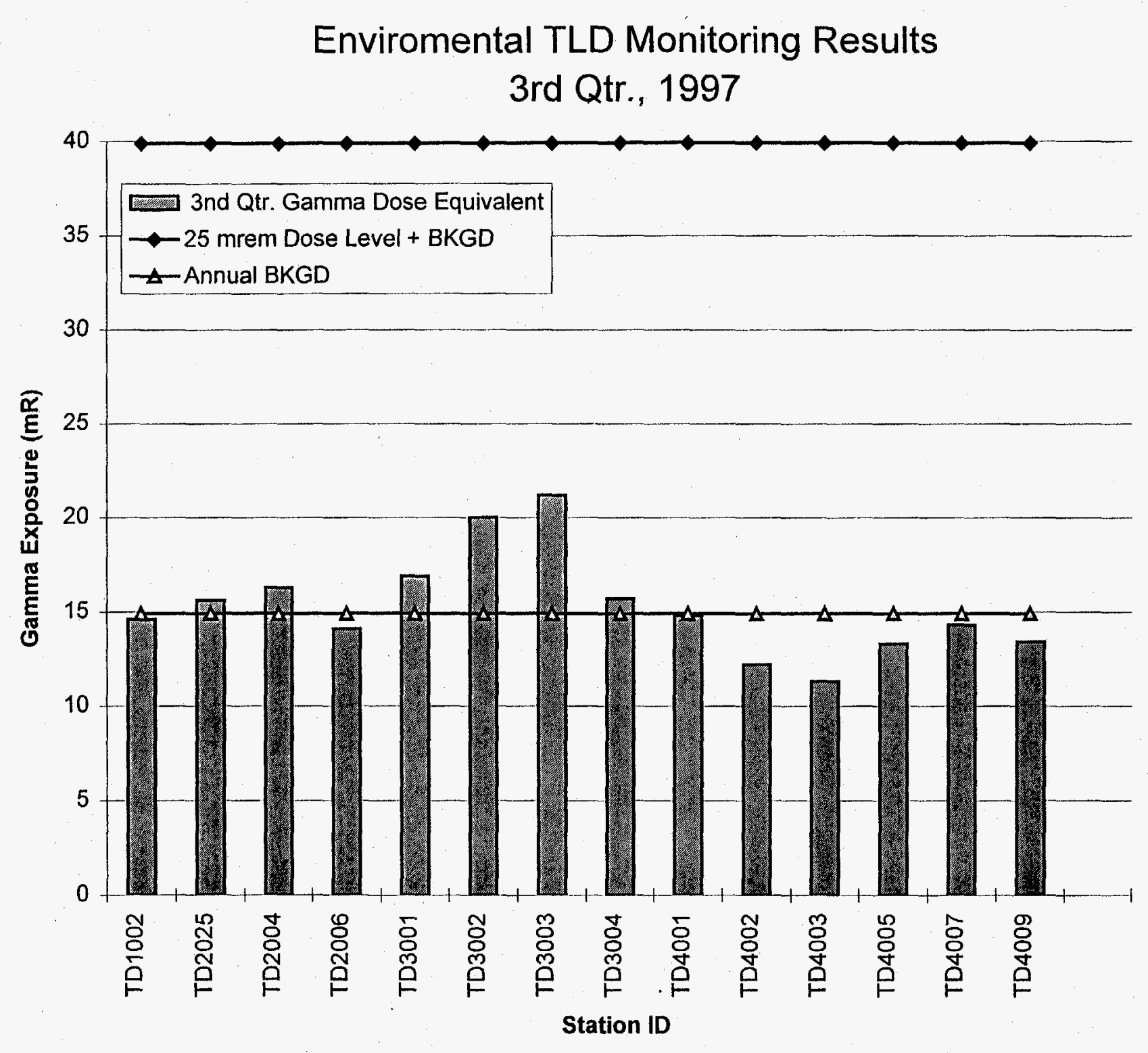




\title{
Fourth Quarter 1997 Air Particulate Results
}

LOCATION ID

\author{
QUARTERLY AVERAGE GROSS \\ ALPHA CONCENTRATION $(\mathrm{uCi} / \mathrm{ml})$
}

STANDARD

NO. WEEKS

DEVIATION

COLLECTED

\begin{abstract}
AP-2001
AP-2002

AP-3003

AP-3004

AP-2005

AP-4006

AP -4007

AP-4008

AP-1009

AP-4011

AP-4012*

AP-4013

AP-3014

AP-1017

AP-2025
\end{abstract}

1.12E-15

1.29E-15

1.41E-15

$1.78 \mathrm{E}-15$

1.37E-15

1.48E-15

1.13E-15

1.29E-15

1.20E-15

1.19E-15

9.75E-16

9.71E-16

1.10E-15

1.21E-15

1.12E-15

3.50E-16 13

4.08E-16 13

5.12E-16 13

9.39E-16 13

6.44E-16 13

4.63E-16 13

4.55E-16 13

4.21E-16 11

4.80E-16 13

$5.66 \mathrm{E}-16 \quad 13$

$7.16 \mathrm{E}-16 \quad 13$

4.71E-16 13

$5.06 \mathrm{E}-16 \quad 13$

4.41E-16 13

3.46E-16 13

* Background monitoring location 


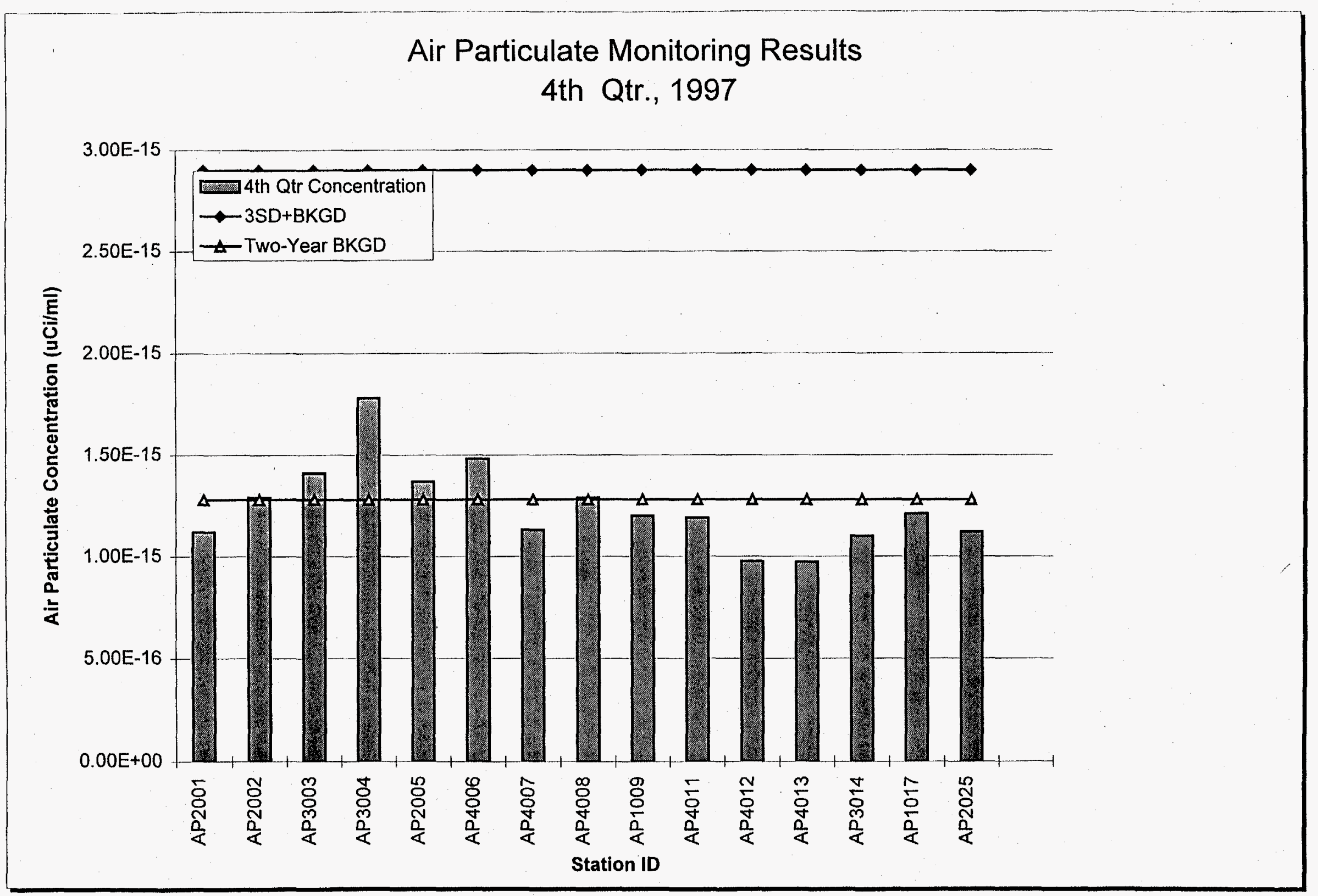

